\title{
Advanced NMR Methodologies in Rock Core Analysis
}

by

Huabing Liu

A thesis

submitted to the Victoria University of Wellington in fulfilment of the requirements for the degree of

Doctor of Philosophy in Physics.

Victoria University of Wellington

2015 



\begin{abstract}
${ }^{1} \mathrm{H}$ NMR techniques have gained extensive acceptance in petrophysics for the evaluation of fluid-saturating reservoir rocks. This thesis presents the development of new NMR methods regarding the reserves (determination of pore length scales and surface relaxivities), productivity (estimates of permeability) and recovery of fluids (resolves of saturation evolution) in rocks.

Traditionally, pore lengths are evaluated from the ground relaxation eigenmodes of spin-bearing molecules in pore space. This evaluation is not straightforward since it is affected by surface relaxivity. Here, we use an approach to determine pore length from detecting the high relaxation eigenmodes, in which way the eigenvalue spectrum directly scales to the pore size distribution. Based on this, we extend this approach for the use with low-field NMR spectrometers and 2D NMR eigenmode correlation methods. Surface relaxivity can be further extracted from these $2 \mathrm{D}$ correlation maps, which is in agreement with an independent NMR measurement.

Permeability is generally estimated from surface relaxation via empirical pore-network models. However, for heterogeneous rocks a single (or averaged) permeability value may not be adequate. Therefore, we measure surface relaxation in conjunction with MRI techniques. Permeability profiles can then be obtained from spatially resolved relaxation maps yielding local connectedness between adjacent slices. The results are confirmed by the comparison of brine-permeability measurements.

MRI experiments of fluids in rocks at reservoir-like conditions may yield optimized recovery strategies of reservoir fluids. In this context we combine MRI with diffusion-relaxation correlation measurements during
\end{abstract}


flooding intervals. The results provide substantial information, such as flooding front and saturation profiles of immiscible fluids discriminated by fluid type. 


\section{Acknowledgments}

Time flies since I arrived to Wellington three years ago. From the day I studied and lived in New Zealand, I start receiving many generous helps from people to whom I wish to express my gratitudes before this thesis.

First of all, I would like to sincerely acknowledge my supervisor Dr. Petrik Galvosas, for your continuous encouragement, guidance and generosity throughout these years. I appreciate that you accepted me as your PhD student without any hesitation. From then on, I benefited quite a lot from your foresight on this $\mathrm{PhD}$ project, your persistence to support my thoughts and also your patience on my junior language. Another important skill I learn from you is how to convey the scientific knowledge for people to easily digest. It is also the highlights of my happy memories that you routinely organize lab cake talks and staff club visits every Friday, which are the chances I know different cultures, customs beyond the science life.

I want to also thank my workmates from NMR lab at Victoria University of Wellington. Dr. Sergei Obruchkov for assisting me to figure out electronic problems and set up the core analysis on $64 \mathrm{MHz}$ NMR system, Dr. Marcel Nogueira d' Eurydice for fruitful discussion on most of the technique development, Dr. Bradley Douglass for introducing cool software and correcting my English grammars, Dr. Stefan Hertel for extensive communication on NMR theory and Tim Brox, as a master of engineering, for significant suggestion and general assistance on hardware modelling and implementation. Dr. Wilfred Kittler, for sharing his knowledge on porous media study. Phillip Luey, as the technician and also a nice mate in 
NMR lab, for helping me to accommodate everything in NZ and always invites me to watch Rugby games. Besides that, thank the workshop fellows, Manu and Nick, for driving my ideas coming to truth.

I would also like to thank enormous supports and collaborations from Magritek researchers. Dr. Robin Dykstra, as my second supervisor, always agrees with me to purchase necessary instrument parts. Dr. Mark Hunter, for providing inspiring suggestions and huge inputs for the implementation of NMR overburden system. Dr. Evan McCarney and Sean Buchanan for sharing their knowledge and experience in NMR petrophysical application.

I'm grateful to meet all new friends in this lovely country and thank you all to make these three years a unforgettable experience in my life.

I sincerely appreciate the financial support from Chinese Scholarship Council to cover my tuition and living fee in New Zealand, and thank Ministry of Business, Innovation and Employment in New Zealand and Victoria University of Wellington for offering me the funding for my $\mathrm{PhD}$ research work. I also take this chance to thank my previous supervisor in China, Prof. Lizhi Xiao, for encouraging me to take this new adventure.

Lastly, I would like to thank my family members in China, who always gave me a solid back when I was distracted by some issues. I want to deeply thank my Fangrong, for accompanying me during the time in VUW, providing me many valuable suggestions and immediate helps to load off my pressure and regain confidence when I met any troubles. I definitely cannot cross this finishing line without you being here. 


\section{Contents}

List of Figures $\quad$ ix

List of Tables $\quad$ xiii

List of Symbols and Abbreviations $\quad$ Xv

1 Introduction 1

2 NMR Fundamentals $\quad 7$

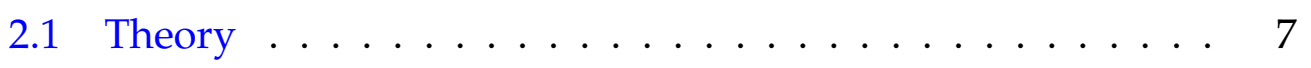

2.1.1 Polarization ............... 7

2.1.2 Excitation and relaxation ........... 10

2.2 Experimental techniques . . . . . . . . . . . . . . . . . . . . . . . . . . . 14

2.2.1 Pulse sequences ingredients . . . . . . . . . . . 14

2.2.2 Free induction decay and spin echo . . . . . . . . 15

2.2.3 Relaxometry . . . . . . . . . . . . . 18

2.2.4 Diffusion . . . . . . . . . . . . . . 21

2.2.5 Magnetic Resonance Imaging . . . . . . . . . . . . . 23

2.3 Multi-dimensional NMR techniques . . . . . . . . . . 26

2.4 Instrumentation . . . . . . . . . . . . . . . . . 28

2.5 Data processing . . . . . . . . . . . . . . . 31

2.5.1 Fourier Transform (FT) . . . . . . . . . . . 31

2.5.2 Inverse Laplace Transform (ILT) . . . . . . . . . . 32 
3 Rock Core Analysis using NMR 37

3.1 Particular consideration for NMR in rocks . . . . . . . . . 37

3.1 .1 Nuclear species . . . . . . . . . . . . . 37

3.1 .2 Magnet strength . . . . . . . . . . . . . 38

3.1.3 Internal magnetic field $B_{i n} \ldots \ldots \ldots$. . . . . 40

3.2 Porosity $\phi \ldots \ldots \ldots \ldots \ldots$. . . . . . . . . . . . 42

3.3 Pore length $a \ldots \ldots \ldots \ldots$. . . . . . . . . . . . 44

3.3.1 Surface relaxation . . . . . . . . . . . . . . 44

3.3.2 High relaxation eigenmode . . . . . . . . . . 46

3.3.3 Other methods . . . . . . . . . . . . 46

3.4 Heterogeneity . . . . . . . . . . . . . . . 47

3.5 Tortuosity $T$ and permeability $\kappa \ldots \ldots . \ldots . . \ldots 48$

3.6 Fluids identification and wettability . . . . . . . . . 51

3.7 Flooding process . . . . . . . . . . . . . 53

4 Pore length and surface relaxivity 55

4.1 Pore length determination . . . . . . . . . . . . 55

4.1.1 Review of pore length determination using the first excited mode $(n=1) \ldots \ldots . \ldots 55$

4.1.2 Strategies of the first excited mode detection in lowfield NMR . . . . . . . . . . . . . 61

4.1.3 Summary of eigenmode involved in 1D NMR . . . . 63

4.2 2D relaxation eigenmode correlation . . . . . . . . . 63

4.2.1 Correlation of ground $T_{2}$ and first $T_{1}$ mode . . . . . 64

4.2.2 Correlation of ground $T_{1}$ and first $T_{1}$ mode . . . . . 67

4.3 Estimation of surface relaxivity . . . . . . . . . . . 73

4.3.1 Estimation of surface relaxivity $\rho_{2} \ldots \ldots \ldots 74$

4.3.2 Estimation of surface relaxivity $\rho_{1} \ldots \ldots \ldots$

4.4 Experimental .................... 75

4.5 Results ..................... . . 76

4.5 .1 1D experimental results . . . . . . . . 76 
4.5.2 $T_{1}-T_{2}$ eigenmode correlation results . . . . . . . 79

4.5.3 $T_{1}-T_{1}$ eigenmode correlation results $\ldots \ldots . \ldots 88$

5 Local Permeability Estimation $\quad 97$

5.1 Methodology . . . . . . . . . . . . . . . 97

5.1.1 Fast encoding $T_{1}$ relaxation . . . . . . . . . 98

5.1.2 Spatially-resolved $T_{1}$ relaxation profile . . . . . . 100

5.1 .3 Permeability profile . . . . . . . . . . . 102

5.2 Experimental . . . . . . . . . . . . . . . . . 104

5.3 Results . . . . . . . . . . . . . . . . 106

5.3.1 Fast encoded $T_{1}$ data and distributions . . . . . . 106

5.3.2 Spatially-resolved $T_{1}$ profiles . . . . . . . . . . 108

5.3 .3 Permeability profiles . . . . . . . . . . . . 110

6 Multi-phase Fluid Flooding 113

6.1 Hardware configuration . . . . . . . . . . . . . . 113

6.1.1 Overburden system . . . . . . . . . . . . 113

6.1.2 Sensitivity for NMR measurements . . . . . . . . 117

6.2 Methodology . . . . . . . . . . . . . . . 118

6.2.1 Spatially-resolved relaxation profiles . . . . . . . 118

6.2.2 Spatially-resolved $D-T_{2}$ correlation maps . . . . . . . 120

6.3 Experimental . . . . . . . . . . . . . . . . . . . 121

6.4 Results . . . . . . . . . . . . . . . . . 125

6.4.1 Porosity profile and fluid distribution . . . . . . . . 125

6.4.2 In-situ water flooding measurement . . . . . . . . . . 129

7 Conclusions and discussions 133 


\section{List of Figures}

1.1 Rock cores analyzed in laboratory . . . . . . . . . . 2

2.1 Schematic of rotating frame . . . . . . . . . . . . . 10

2.2 Excitation and relaxation in the rotating frame . . . . . . 12

2.3 An RF pulse . . . . . . . . . . . . . . . . . . . . . 15

2.4 A pulsed field gradient . . . . . . . . . . . . . . . 15

2.5 Pulse sequence for detecting FID signal . . . . . . . . . . 16

2.6 Pulse sequence for detecting spin echo . . . . . . . . . . . 16

2.7 Schematic of spin echo . . . . . . . . . . . . . . . 17

$2.8 T_{1}$ relaxation pulse sequence and result $\ldots \ldots$. . . . . . . 19

$2.9 T_{2}$ relaxation pulse sequence and result . . . . . . . . . 20

2.10 PGSE pulse sequence and its results in different liquids . . . 23

2.11 Frequency and phase encoded imaging pulse sequences . . . 25

2.12 Schematic of an NMR analyzer. . . . . . . . . . . . . . . 30

2.13 An example of 2D time-domain data and its FT result . . . . 32

2.14 An example of 2D time-domain data and its ILT result . . . . 35

3.1 NMR instruments for rock core analysis in VUW . . . . . . . 39

3.2 Internal magnetic fields in rock pore space . . . . . . . . 42

3.3 Porosity definition and NMR porosities compared to weighing values ...................... 43

3.4 Surface relaxation mechanism and their distributions in rock cores ...................... 45 
3.5 Heterogeneity of rock cores . . . . . . . . . . . . . 48

3.6 Schematic of tortuosity and its determination from timedependent diffusion measurement . . . . . . . . . . . . . 49

3.7 Empirical modes to estimate permeability using NMR relaxation distributions . . . . . . . . . . . . . . . 51

3.8 Schematic of wettability definition and its determination from $2 \mathrm{D} D-T_{2}$ correlation distribution $\ldots . . \ldots 52$

4.1 An example of eigenvalues and relative intensities of eigenmodes in sphere . . . . . . . . . . . . . . . 57

4.2 1D pulse sequence to obtain high eigenmode and pore length scales . . . . . . . . . . . . . . . . 58

4.3 2D NMR pulse sequence used to correlate the ground $T_{2}$ and first excited $T_{1}$ eigenmode . . . . . . . . . . . . . . 65

4.4 2D NMR pulse sequence used to correlate the ground and first excited $T_{1}$ eigenmode . . . . . . . . . . . . . 69

4.5 2D NMR pulse sequence used to validate the ground and first excited $T_{1}$ mode correlation experiments . . . . . . . 72

4.6 1D dataset and pore length scales of Parker sandstone . . . . 77

4.7 1D dataset and pore length scales of Winterset limestone . . 78

4.8 1D dataset and distribution of Nugget sandstone at $64 \mathrm{MHz} \quad 80$

4.9 2D dataset of first $T_{1}$ and ground $T_{2}$ mode correlation in sandstone ...................... 81

4.10 Correlation distributions of $F\left(\tau_{1}^{1}, \tau_{2}^{0}\right)$ and $F\left(\tau_{1}^{0}, \tau_{2}^{0}\right)$ in sandstone .......................... 81

$4.112 \mathrm{D}$ dataset of first $T_{1}$ and ground $T_{2}$ mode correlation in limestone . . . . . . . . . . . . . . . 82

4.12 Correlation distributions of $F\left(\tau_{1}^{1}, \tau_{2}^{0}\right)$ and $F\left(\tau_{1}^{0}, \tau_{2}^{0}\right)$ in limestone 83

4.13 Correlation functions $F\left(a, T_{2}\right)$ of sandstone and limestone . 84

$4.14 T_{2}(a)$ curves in sandstone and limestone . . . . . . . . . 86

4.15 Padé approximation on correlation functions $F\left(D, T_{2}\right)$ of sandstone and limestone . . . . . . . . . . . . 88 
4.16 Raw distributions of ground and first $T_{1}$ mode correlation in sandstone ................... 89

4.17 Raw distributions of ground and first $T_{1}$ mode correlation in limestone . . . . . . . . . . . . . . . . . 990

4.18 2D dataset of first and ground $T_{1}$ mode correlation in sandstone 92

$4.192 \mathrm{D}$ dataset of first and ground $T_{1}$ mode correlation in limestone 93

$4.202 \mathrm{D}$ ground and high $T_{1}$ mode correlation distribution in sandstone and limestone . . . . . . . . . . . . . . . . 94

4.21 Correlation functions $F\left(a, T_{1}\right)$ in sandstone and limestone . 95

$4.22 T_{1}(a)$ curves in sandstone and limestone . . . . . . . . . . 95

5.1 Pulse sequence for rapid encoding $T_{1} \ldots \ldots \ldots$. . . . . 98

5.2 Pulse sequence for spatially resolved $T_{1}$ mapping $\ldots$. . . . 100

5.3 A proposed model to calculate local permeability connected factor $P^{i, i+1} \ldots \ldots \ldots \ldots$. . . . . . . . . . 104

5.4 Rock samples used and their 2D MRI imaging results. . . . . 105

5.5 Fast encoding and inversion-recovery $T_{1}$ comparison in the sandstone sample. . . . . . . . . . . . . . . . . 107

5.6 Fast encoding and inversion-recovery $T_{1}$ comparison in the limestone sample. . . . . . . . . . . . . . . . . . . 108

5.7 Spatially resolved $T_{1}$ mappings in the sandstone and limestone cores. . . . . . . . . . . . . . . . . . . . . . . 109

5.8 Permeability profiles in the sandstone and limestone cores. . 111

6.1 Schematic (a) and detailed parts (b) of overburden rock holder114

6.2 Schematic of the overburden rock holder combined with the low-field NMR system . . . . . . . . . . . . . . . . . 115

6.3 Sensitivity and stability test of NMR overburden system at different temperatures . . . . . . . . . . . . 118

6.4 NMR pulse sequence for spatially resolved $T_{2}$ profiles using phase encoded method . . . . . . . . . . . . . . . . 119

6.5 NMR pulse sequence for spatially resolved $D-T_{2}$ map $\ldots . .121$ 
6.6 Petrophysical information of core sample used in flooding experiment . . . . . . . . . . . . . . . 122

6.7 $1 \mathrm{D} T_{2}$ and $T_{1}$ relaxation distributions of oil-bearing core at different temperatures . . . . . . . . . . . . 126

6.8 2D $D-T_{2}$ distributions of oil-bearing core at different temperatures . . . . . . . . . . . . . . . . 127

6.9 Spatially resolved relaxation profiles of oil-bearing core at different temperatures . . . . . . . . . . . . . . 128

6.10 Spatially resolved $D-T_{2}$ profiles of oil-bearing core before and after water flooding . . . . . . . . . . . . . 129

6.11 Relative water and oil saturation during flooding process . . 130

6.12 Projected $D-T_{2}$ correlation distribution at different temperatures ......................... 131

6.13 Projected spatially resolved $D$ profiles at different temperatures132 


\section{List of Tables}

2.1 Categories of multi-dimensional NMR techniques. . . . . . . 29

3.1 Nuclear species used in NMR rock core analysis . . . . . . . 38

4.1 Phase cycling in 1D pore length scales determination . . . . 62

4.2 Eigenmode measured in different NMR pulse sequences . . 63

4.3 Phase cycling for $2 \mathrm{D}$ ground $T_{2}$ and first $T_{1}$ mode correlation pulse sequence . . . . . . . . . . . . . 66

4.4 Phase cycling for $2 \mathrm{D}$ ground and first $T_{1}$ mode correlation pulse sequence . . . . . . . . . . . . . 70

4.5 Phase cycling for validating $2 \mathrm{D}$ ground and first $T_{1}$ mode correlation pulse sequence . . . . . . . . . . . 73

5.1 Phase cycling for phase encoded $T_{1}$ imaging $\ldots \ldots$. . . . . 101

6.1 Experimental test of temperature and pressure conditions in overburden system. . . . . . . . . . . . . . 117 


\section{List of Symbols and}

\section{Abbreviations}

$\alpha$

$\delta \chi$

$\Delta z$

$\gamma$

$\hbar$

$\kappa$

$\nu$

$\omega_{0}$

$\phi$

$\theta$

$\xi$

$B_{0}$

$B_{i n}$

D
Tip angle

Susceptibility contrast

Imaging resolution

Gyromagnetic ratio

$\frac{h}{2 \pi}$

Permeability

Volume relaxivity

Larmor frequency

Porosity

contact angle in multi-fluid/solid surface system

Spin density

Static magnetic field

Internal magnetic fields

Self-diffusion coefficient 


\begin{tabular}{ll} 
xvi & \multicolumn{1}{c}{ LIST OF SYMBOLS AND ABBREVIATIONS } \\
$F$ & Electrical formation factor \\
$k_{B}$ & Boltzmann constant \\
$M_{0}$ & Net magnetization \\
$T$ & Tortuosity \\
$T_{1}$ & Longitudinal relaxation time \\
$T_{2}$ & Transverse relaxation time \\
$T_{2}^{*}$ & Transverse relaxation time due to inhomogeneities \\
$T_{\mathrm{E}}$ & Echo spacing \\
$T_{2 l m}$ & logarithmic mean value of $T_{2}$ distribution \\
BRD & Butler-Reeds-Dawson \\
BVI & Bulk Volume Irreducible \\
COSY & COrrelation SpectroscopY \\
CPMG & Carr-Purcell-Meiboom-Gill \\
dB & deciBels \\
DDCOSY & Diffusion-Diffusion COrrelation SpectroscopY \\
DEXSY & Diffusion EXchange SpectroscopY \\
DOSY & Diffusion-Ordered SpectroscopY \\
DRCOSY & Diffusion-Relaxation Correlation SpectroscopY \\
DRICOSY & Diffusion-Relaxation-Internal Gradient Correlation Spec- \\
ESR & Electron Spin Resonance \\
&
\end{tabular}


FFI

FID

FOV

FT

ILT

LT

MCF

MMME

MRI

MRS

NMR

PDE

PFG

PGSE

PGSTE

POXSY

PV

REXSY

RF

RICOSY

RMS
Free Fluid Index

Free Induction Decay

Field Of View

Fourier Transform

Inversion Laplace Transform

Laplace Transform

Multiple Correlation Function

Multiple Modulation Multiple Echoes

Magnetic Resonance Imaging

Magnetic Resonance Sounding

Nuclear Magnetic Resonance

Partial Differential Equation

Pulse Field Gradient

Pulse Gradient Spin Echo

Pulse Gradient STimulated Echo

Positional EXchange SpectroscopY

Pore Volume

Relaxation EXchange SpectroscopY

Radio Frequency

Relaxation-Internal gradients Correlation SpectroscopY

Root Mean Square 
xviii LIST OF SYMBOLS AND ABBREVIATIONS

RRCOSY Relaxation-Relaxation Correlation SpectroscopY

RROSY Relaxation-Relaxation-Ordered SpectroscopY

SNR Signal-to-Noise ratios

SVD Singular Value Decomposition

VEXSY Velocity EXchange SpectroscopY 


\section{Chapter 1}

\section{Introduction}

As a typical but complex porous material, rock core provides essential data for the exploration, evaluation and production of reservoirs in petroleum, geothermal and hydrological industries. These rock samples allow geoscientists to examine the first-hand depositional sequences penetrated by a drill bit [1,2]. They offer direct evidence of the presence, distribution and deliverability of geothermal gas, ground water, hydrocarbons and can reveal variations in reservoir traits that might not be detected through down-hole logging measurements alone.

Plenty of methods have been developed to study the rock samples regarding the relationship between their physical responses and corresponding petrological properties [3, 4]. Those methods can be categorized into two classes. The first type adopts direct imaging pattern, such as Magnetic Resonance Imaging (MRI), Scanning Electron Microscopy (SEM), thin-section optical micrography and X-ray computed-tomography (CT). These techniques can visualize the interior solid matrix structure or fluid distribution of the core samples straightforwardly. The second type, on the other hand, relies on indirect imaging techniques, for instance Small-Angle Neutron Scattering (SANS), gas adsorption isotherms, Mercury Intrusion Porosimetry (MIP), Nuclear Magnetic Resonance (NMR), Electron Spin Resonance (ESR), electrical resistivity, and acoustic waveform, therefore, 
yield the volumetric properties of rock cores.

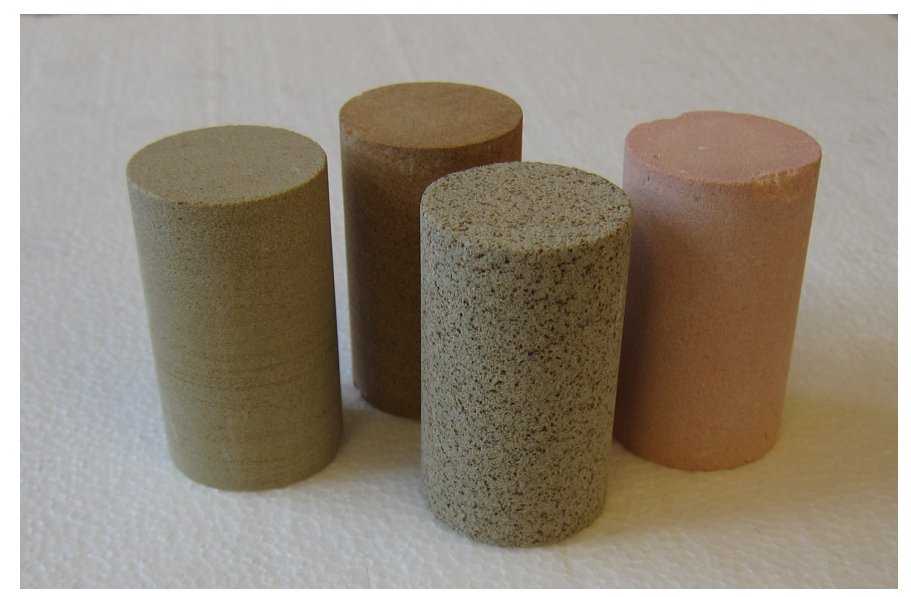

FIG. 1.1: 1.5 inch rock cores with different lithologies studied in laboratory. Photo provided by Mark Hunter from Magritek Ltd.

During this PhD project, NMR has continued its development as an unique tool for investigating rock cores, to unravel the morphology features and the hierarchy of interactions with fluids confined in its pore space. It is one of the non-invasive techniques to offer "body check" for rock samples and deliver substantial petrophysical parameters [5-8]. In this context, relaxation time/diffusion coefficient distributions, as well as their multi-dimensional correlation maps, are routinely utilized to characterize pore size, wetting state, fluids types, and quantify individual fluid phase saturations [9-11]. Meanwhile, MRI was introduced to provide insight of the presence of fluid bearing in the pore network and monitor "real-time" fluid invasion profiles during core flooding experiments [6, 12].

In this thesis, three important characteristics, pore length scales, permeability and fluid saturation profile, with respect to the reserves, productivity and recovery of the rocks, are studied respectively. Before involving into any technical details of the developed methods in this thesis, a brief review of NMR and its application in rock core analysis is given in Chapter 2 and Chapter 3.

Chapter 2 presents NMR principle and fundamentals, including some 
necessary concepts (for instance relaxation and diffusion), experimental techniques, instrument and data processing protocols. Many emerging MR methods relevant to the topic of this thesis, such as fast $T_{1}$ encoding and $D$ encoding, and multi-dimensional NMR techniques, are briefly introduced or summarized in this chapter. Afterwards, Chapter 3 outlines the current study of NMR application in rock core analysis. Two special consideration, nuclear species and magnet types used in MR rock core analysis are addressed first. Various petrophysical parameters, including porosity, pore length, heterogeneity degree, tortuosity, permeability, wettability as well as the fluid transport, can be obtained by using the techniques developed in NMR context.

Based on the knowledge from Chapter 2 and Chapter 3, we start to introduce the methods we developed and the hardware we implemented from Chapter 4 to Chapter 6.

Pore length is a critical structural parameter describing the dimension of the pores preserving reservoir fluid in porous rocks [1]. Routine MR techniques, such as surface relaxation distributions and time-dependent diffusion coefficient measurement, are utilized to determine pore lengths of rock cores [5, 13-15]. However, surface relaxation measurements are normally convoluted with surface relaxivities, which makes themselves indirect to determine pore length scales. Time-dependent diffusion coefficient measurement provides a mean value of pore size in rock, which is probably inadequate to describe the complex pore structure. Therefore, it is necessary to develop and implement a method which is capable to measure pore length distribution straightforwardly. Chapter 4 presents the theory and experimental results on pore length scales of rock cores by detecting the high eigenmodes of spin bearing molecules satisfying the diffusion equation in the presence of internal magnetic fields $B_{i n}$. Since higher relaxation eigenmodes (with mode index $n>0$ ) are ascribed to the molecules diffusion process in pore space, it provides a direct access to pore length $a$. This method can be also extended to low-field NMR context 
by optimizing the encoding time for molecules experiencing strong enough modulation in pore space. Furthermore, this methodology was combined with relaxometry measurements into a two-dimensional experiment, which provides correlation between pore length and relaxation time. This correlation technique allows one to extract information on the surface relaxivity of the rock cores. The estimated surface relaxivities are then compared and confirmed by the values from an independent NMR method.

Permeability is a function measuring the ease with which a fluid flows through a porous media and is an important input to model the reservoir behavior to optimize the underground resources exploration. It is generally predicted from surface relaxation distribution via empirical pore-network models [5, 16-22]. However, for some heterogeous rocks a single (or averaged) permeability value may not be adequate to reflect the permeability variation in some local regimes. In order to improve the permeability evaluation of heterogeneous rock cores, Chapter 5 introduces a customized spatially-resolved NMR relaxation profile technique. A fast relaxation encoding method was firstly implemented in the field as low as $2 \mathrm{MHz}$. Later on, a phase encoded gradient was applied in conjunction with the aforementioned fast relaxation method. Two permeability models were subsequently utilized to interpret the obtained spatially-resolved relaxation map to obtain the permeability profile. One was directly applying empirical formula consecutively along the imaging direction. The other model evaluates the permeability profile by further considering a proposed local connectedness factor between the adjacent resolved imaging slices. The results from both models can reveal the variation of heterogeneity and permeability of rock plugs. Finally, by comparing these results with independent brine permeability values in different types of rock samples, it is advisable to adopt the later model when trying to understand the local permeability of rock samples.

Most reservoir rocks are buried in the formation approximately thousands meter deep, where the in-situ temperatures and pressures are much 
higher as compared to conditions on the surface ground $[2,23]$. In this case, fluid properties (e.g. viscosity) in porous rocks depend strongly on temperature and pressure, leading to a complex mechanism of oil recovery in oilfields [1]. Therefore, in order to characterize fluid properties and model fluid transport, it is necessary to provide reservoir-like temperatures and pressures whilst the experiments are performed in laboratory measurements. Moreover, it is desirable and meaningful to understand the reservoir fluid transport and its recovery mechanism within more than one fluid phase. In this case, although it is routinely applied to differentiate fluid types and evaluate the relative saturation, 2D diffusion-relaxation correlation experiment fails to monitor the fluid displacement and flooding front since it is a volumetric method [9, 11, 24-26]. Chapter 6 introduces the work on setting up an overburden system and analyzing the rock plugs on emerging NMR instrument at reservoir-like conditions. 1D NMR relaxation and 2D diffusion-relaxation correlation distributions of fluid-saturated rock cores were measured in conjunction with 1D NMR imaging. By performing these spatially resolved NMR relaxometry and diffusometry experiments during flooding multi-phase fluids through the rock core, it is possible to obtain porosity, fluid saturation and residual fluid content profiles under reservoir-like conditions. Moreover, by tuning the surroundings of the studied sample ( for instance temperatures) and quantitatively estimate the relative saturation of individual fluid from the spatially-resolved $D-T_{2}$ experimental results performed with this NMR setup, it may inform fluid recovery enhancement strategies during the flooding process.

Finally, conclusions regarding the methods developed during this $\mathrm{PhD}$ works and its application to rock cores are given in the Chapter 7 of this thesis. 


\section{Chapter 2}

\section{NMR Fundamentals}

As an unique technique developed for 70 years, Nuclear Magnetic Resonance (NMR) has contributed four Nobel prizes in the progress of multiple disciplines. Since utilizing NMR as major protocol in this thesis, the necessary fundamentals of NMR, from its theory and pulse sequences, to instruments as well as data processing are briefly reviewed in this chapter.

\subsection{Theory}

\subsubsection{Polarization}

NMR phenomenon can be observed in atomic nuclei that possess a nonzero spin angular momentum $\hbar \mathbf{I}$ and a collinear dipolar magnetic moment $\mu$ described as [27]:

$$
\mu=\gamma \hbar \mathbf{I}
$$

Here $\gamma$ is gyromagnetic ratio and $\hbar$ is Planks constant divided by $2 \pi$. I is the dimensionless angular momentum and $\mathbf{I}^{2}$ has eigenvalues $I$ which are either integer or half-integer.

By placing the atomic nuclei in an external magnetic field $B_{0}$ applied along the $z$-axis in the laboratory frame of reference, the energy splits due 
to Zeeman interaction. For a simple quantum mechanical description, the Hamiltonian of the spin system in individual energy state is given by [27]:

$$
\mathcal{H}=-\gamma \hbar B_{0} I_{z}
$$

where $I_{z}$ is the $z$ component of $\mathbf{I}$. The eigenvalues of this Hamiltonian is any one of a discrete set of integer or half-integer values in the range $I$, $I-1, \ldots,-I$ and one can easily find that there exists $2 I+1$ allowed energy levels. Since we exclusively study ${ }^{1} \mathrm{H}$ nucleus with $I=\frac{1}{2}$ in this thesis, there are only two energy states and the energy gap between these two states is:

$$
\Delta E=\gamma \hbar B_{0}
$$

Within assemblies consisting of a large number of nuclear spins, the populations of the spins precessing along $B_{0}$ (referred to as the case of low energy state) and against $B_{0}$ (referred to as the case of high energy state) are not identical and the ratio of the final equilibrium populations in these two states can be described by Boltzmann distribution [28]:

$$
\frac{n_{\downarrow}}{n_{\uparrow}}=\exp \left(-\frac{\Delta E}{k_{B} T}\right)=\exp \left(-\frac{\gamma \hbar B_{0}}{k_{B} T}\right)
$$

where $n_{\downarrow}$ and $n_{\uparrow}$ represent the populations of the spins belong to high and low energy levels, respectively. $k_{B}$ is the Boltzmann constant $\left(=1.381 \times 10^{-23} \mathrm{~J} / \mathrm{K}\right)$ and $T$ is the temperature. The excess occupation in the lower energy state leads to an excess of magnetic moments oriented along $B_{0}$, thus building up a macroscopic magnetization $M_{0}$. Therefore it can be calculated by summing up all the possible magnetic moments $\mu$ in the spin $\frac{1}{2}$ system by:

$$
M_{0}=\sum_{i=1}^{N_{0}} \mu_{i z}=n_{\uparrow} \mu_{z \uparrow}+n_{\downarrow} \mu_{z \downarrow}=\frac{\left(n_{\uparrow}-n_{\downarrow}\right)}{2} \gamma \hbar
$$

where $N_{0}$ stands for the total spin population in the system and $\mu_{z}$ is the 
$z$-component of the magnetic moment.

By using Taylor expansion of the Boltzmann exponential under the condition of the high temperature approximation $\left(k_{B} T \gg \Delta E\right)$, Eq. 2.4 can be easily simplified to be $\frac{n_{\downarrow}}{N_{0}-n_{\downarrow}} \approx 1-\frac{\gamma \hbar B_{0}}{k_{B} T}$, and therefore:

$$
n_{\downarrow} \approx \frac{\frac{N_{0}}{2}\left[1-\frac{\gamma \hbar B_{0}}{k_{B} T}\right]}{1-\frac{\gamma \hbar B_{0}}{2 k_{B} T}} \approx \frac{\frac{N_{0}}{2} \exp \left(-\frac{\gamma \hbar B_{0}}{k_{B} T}\right)}{\exp \left(-\frac{\gamma \hbar B_{0}}{2 k_{B} T}\right)} \approx \frac{N_{0}}{2}\left[1-\frac{\gamma \hbar B_{0}}{2 k_{B} T}\right]
$$

Similarly, we can derive the population of spins along $B_{0}$ to be:

$$
n_{\uparrow} \approx \frac{N_{0}}{2}\left[1+\frac{\gamma \hbar B_{0}}{2 k_{B} T}\right]
$$

Therefore, the population difference of the spins in these two energy levels $\Delta N$ would be:

$$
\Delta N=n_{\uparrow}-n_{\downarrow} \approx \frac{N_{0}}{2}\left[\frac{\gamma \hbar B_{0}}{k_{B} T}\right]
$$

By substituting Eq. 2.8 into Eq. 2.5, the net magnetization in the spin $\frac{1}{2}$ system $M_{0}$ can then be expressed as:

$$
M_{0}=N_{0} \frac{\gamma^{2} \hbar^{2} B_{0}}{4 k_{B} T}
$$

It is worth mentioning that $M_{0}$ is the source of all NMR signal which will be manipulated and eventually detected during NMR experiments. Since it is derived from the individual spins rotating around $B_{0}$, the net magnetization $M_{0}$ will also precess around $B_{0}$ and its precessing angular frequency is given by:

$$
\omega_{0}=\gamma B_{0}
$$

where $\omega_{0}$ is called the Larmor frequency. 


\subsubsection{Excitation and relaxation}

In order to observe the NMR phenomenon, the spins need to be perturbed away from the steady state after their polarization. Spins can be excited by irradiating the sample with photons of the same energy as given in Eq. 2.3. Currently this is achieved by applying an independently oscillating magnetic field $B_{1}$ perpendicular to the static magnetic field $B_{0}$. In particular, $B_{1}$ needs to precess along the same direction of $B_{0}$ and with a frequency, $\omega_{B_{1}}$, same to the Larmor frequency $\omega_{0}$ in order to effectively manipulate the $M_{0}$ vector $^{1}$.

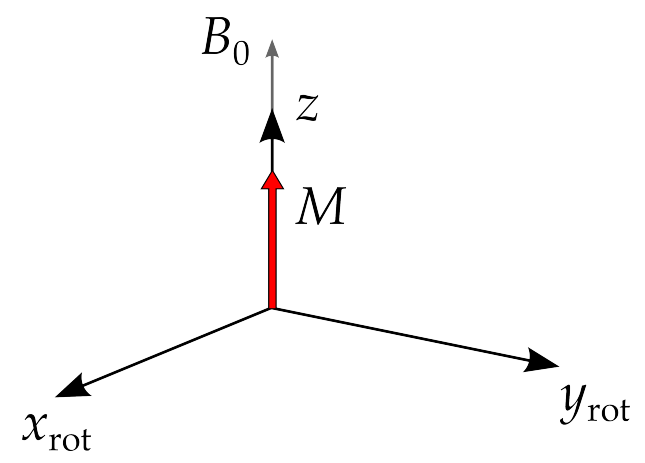

FIG. 2.1: Schematic of rotating frame.

For the purpose of visualizing this excitation process in a more convenient way, a frame of reference, different from the laboratory frame, named rotating frame, is utilized and shown in Fig. 2.1. In this new frame, $z, x_{\text {rot }}$ and $y_{\mathrm{rot}}$ are considered rotating with an angular frequency of $\omega_{0}$, therefore, the precession of $M_{0}$ is no need to be considered. Consequently, the emitted $B_{1}$ will be stationary in the transverse plan to $B_{0}$ and its excitation or manipulation to $M_{0}$ can be illustrated using vector model, which simplifies a sophisticated quantum mechanical phenomenon through a classical view-

\footnotetext{
${ }^{1}$ The applied alternating field $B_{1}$ is a linearly polarized field with a pattern of $2 B_{1} \cos \left(\omega_{B_{1}} \mathrm{t}\right)$. For easily understanding its interaction with $M_{0}$, it is considered as superimposition of $B_{1} \exp \left(i \omega_{B_{1}} t\right)$ and $B_{1} \exp \left(-i \omega_{B_{1}} t\right)$, where the first component will be responsible for the resonance phenomenon when $\omega_{B_{1}}=\omega_{0}$, whereas the second one is counter-rotating and can be neglected in the condition of $B_{1} \ll B_{0}$ [28].
} 
point. In this thesis, we stick to use the rotating frame when discussing the manipulation of the net magnetization $M_{0}$ and the axes of the rotating frame, $z, x_{\text {rot }}$ and $y_{\text {rot }}$ are referred to as $z, x$ and $y$.

With the concepts of net magnetization vector $M_{0}$ and rotating frame at hand, the Bloch equations can be employed to describe the manipulation (excitation) and time evolution (relaxation) process of magnetization according to the classical torque theory [29]:

$$
\frac{d \mathbf{M}}{d t}=\gamma \mathbf{M}(t) \times \mathbf{B}(t)-\mathbf{R}(\mathbf{M}(t)-\mathbf{M}(0))
$$

where $\mathbf{M}, \mathbf{B}$ and $\mathbf{R}$ can be considered as vectors and matrices. The elements in $\mathbf{M}$ are $\left[\begin{array}{l}M_{x} \\ M_{y} \\ M_{z}\end{array}\right]$, which represent the magnetization components in different directions. The elements in $\mathbf{B}$ are $\left[\begin{array}{c}B_{x} \\ B_{y} \\ 0\end{array}\right]$, standing for the magnetic field components. The third element is 0 instead of $B_{0}$ here since the rotation along $B_{0}$ is neglected in the rotating frame with the resonant condition of $\omega_{B_{1}}=\omega_{0}$. $\mathbf{R}$ matrix determines the relaxation process of the magnetization vector. The elements in $\mathbf{R}$ are $\left[\begin{array}{ccc}1 / T_{2} & 0 & 0 \\ 0 & 1 / T_{2} & 0 \\ 0 & 0 & 1 / T_{1}\end{array}\right]$. Here $T_{2}$ characterizes the relaxation process of the transverse magnetization components and $T_{1}$ for the longitudinal magnetization component. The details of $T_{2}$ and $T_{1}$ will be discussed later.

By applying an oscillating field $B_{1}$ in the transverse plane of the rotating frame (shown in Fig. 2.2 (a)), the effective $\mathbf{B}$ vector will be $\left[B_{1, x} ; B_{1, y} ; 0\right]$, where $B_{1, x}$ and $B_{1, y}$ are the projection of $B_{1}$ along $x$ and $y$ axes. The solution 
of Eq. 2.11 in this case can be given as:

$$
\begin{aligned}
& M_{x}(t)=M_{0} \cdot \cos \left[\arctan \left(\frac{B_{1, y}}{B_{1, x}}\right)\right] \cdot \sin \left(\gamma B_{1} t\right) \\
& M_{y}(t)=M_{0} \cdot \sin \left[\arctan \left(\frac{B_{1, y}}{B_{1, x}}\right)\right] \cdot \sin \left(\gamma B_{1} t\right) \\
& M_{z}(t)=M_{0} \cdot \cos \left(\gamma B_{1} t\right)
\end{aligned}
$$

in the initial condition that $\mathbf{M}(0)=\left[0 ; 0 ; M_{0}\right]$. Here the effect from the second term in Eq. 2.11 during the evolution time $t$ is ignored.

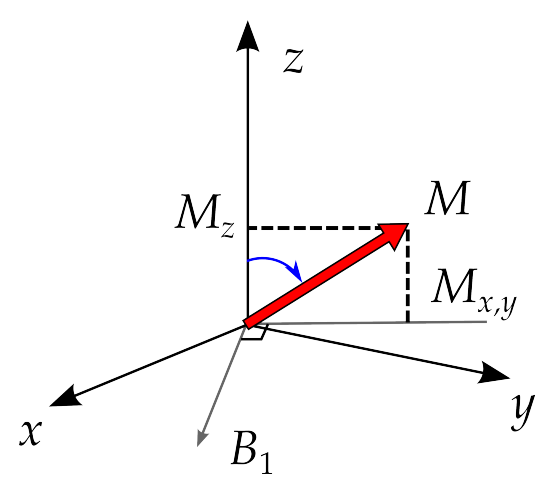

(a)

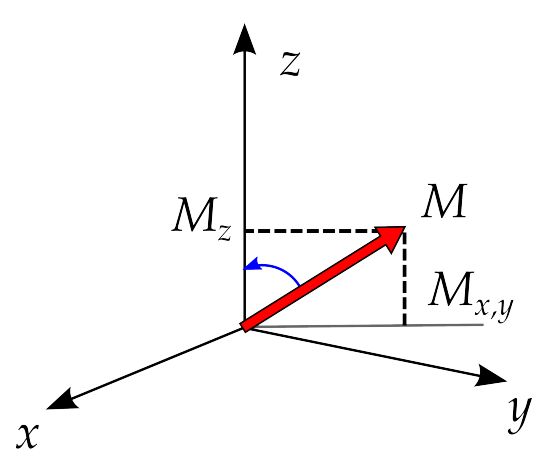

(b)

FIG. 2.2: Schematic of excitation (a) and relaxation (b) in the rotating frame.

If the emission of $B_{1}$ field is stopped, the spin system prefers to recover to the thermal equilibrium state. From the view of magnetization vector, it will precess along $B_{0}$ and be driven back to $z$-axis. Again, considering $\mathbf{B}=[0 ; 0 ; 0]$ in Eq. 2.11, the solution during this procedure is determined by $T_{2}$ and $T_{1}$ in matrix $\mathbf{R}$, which characterize the time of magnetization decay in the transverse plan and recovery along $z$-axis, respectively. Therefore, 
the individual components in Eq. 2.11 in this case will be:

$$
\begin{aligned}
\frac{d M_{x}}{d t} & =-\frac{M_{x}}{T_{2}} \\
\frac{d M_{y}}{d t} & =-\frac{M_{y}}{T_{2}} \\
\frac{d M_{z}}{d t} & =\frac{M_{0}-M_{z}}{T_{1}} .
\end{aligned}
$$

The solutions of these above equations are given as:

$$
\begin{aligned}
& M_{x}(t)=M_{x}(0) \exp \left(-\frac{t}{T_{2}}\right) \\
& M_{y}(t)=M_{y}(0) \exp \left(-\frac{t}{T_{2}}\right) \\
& M_{z}(t)=M_{z}(0) \exp \left(-\frac{t}{T_{1}}\right)+M_{0}\left[1-\exp \left(-\frac{t}{T_{1}}\right)\right]
\end{aligned}
$$

where the initial magnetization along each direction, $M_{x}(0), M_{y}(0)$ and $M_{z}(0)$ can be obtained from the final solution of Eq. 2.12.

$T_{1}$, also called the spin-lattice relaxation time, corresponds to the process of establishing (or re-establishing) the spin population distribution according to the thermodynamic equilibrium and therefore the net magnetization in the magnetic field. $T_{2}$, which is also known as spin-spin relaxation time, determines the loss of phase coherence among nuclei and therefore the magnetization decay rate in the transverse plane. Note that the return of magnetization to the $z$-direction inherently causes the loss of magnetization in the $x-y$ plane, as a result, $T_{1} \geqslant T_{2}$ in any spin system. The details of measuring these two relaxation times during NMR experiments will be introduced in Sec. 2.2.3.

So far, we consider a uniform $B_{0}$ distributed across the sample space. However, in most situations, this is not true because of the $B_{0}$ distortion due to the properties of magnets or samples. Thus, the magnetization in an elementary volume will process within a local frequency $\omega_{\text {local }}$ proportional 
to the local field strength $B_{0, \text { local }}$ according to Eq. 2.10. As a result, the spin precession dephases rapidly and the transverse magnetization decay in the $x$ - $y$ plane is accelerated. A characteristic time $T_{2}^{*}$, so-called transverse relaxation time due to the field inhomogeneities, is utilized to describe this procedure and is given as:

$$
\frac{1}{T_{2}^{*}}=\frac{1}{T_{2}}+\frac{1}{T_{2}^{\text {Inhomo }}}
$$

Therefore, a relationship of $T_{2} \geqslant T_{2}^{*}$ can be readily concluded from Eq. 2.15.

\subsection{Experimental techniques}

\subsubsection{Pulse sequences ingredients}

As discussed in Sec. 2.1.2, an oscillating magnetic field $B_{1}$ is necessary for observing NMR phenomenon. This field is realized by applying a pulse in Radio-Frequency (RF) range ${ }^{2}$. For further encoding the magnetization phase in the transverse plane, additional pulses named as Pulsed Field Gradients (PFG) can be utilized. A number of these two elements, which are arrayed and ordered in a so-called NMR pulse sequence, are generally applied to study NMR characteristics of the spin systems, for instance $T_{1}$, $T_{2}, T_{2}^{*}$ or diffusion.

As illustrated in Fig. 2.3, an RF pulse is emitted to tip the net magnetization $M_{0}$ from $z$ axis to a certain angle. The tip angle $\alpha$ is determined by the product of gyro-magnetic ratio $\gamma$, field strength $B_{1}$ and pulse duration $t_{\mathrm{p}}, \alpha=\gamma B_{1} t_{\mathrm{p}}$ according to Eq. 2.12. The orientation of RF pulse can be manipulated by choosing the pulse phase accordingly .

PFG pulses are essential in spatial imaging and diffusion experiments

\footnotetext{
${ }^{2}$ The frequency falls into microwave frequency (typically $\mathrm{GHz}$ ) for Electron Spin Resonance (ESR)
} 


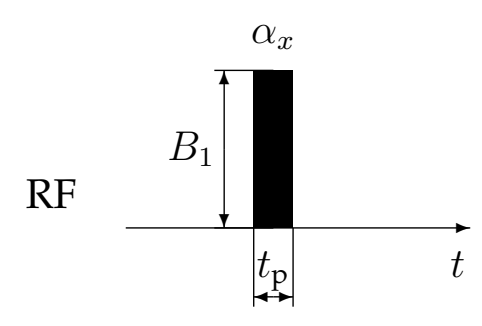

(a)

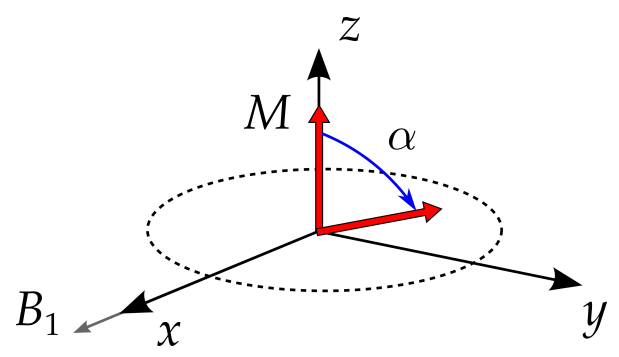

(b)

FIG. 2.3: (a) An RF pulse rotating magnetization vector by an angle of $\alpha$ along the $x$-axis. (b) Magnetization vector tipped by RF pulse.

to encode the spin phase. To apply this pulse across the sample, an extra magnetic field is superimposed to $B_{0}$, and its components in different directions are given as $\mathbf{G}=\left[G_{x} ; G_{y} ; G_{z}\right]=\left[\frac{\partial B_{z}}{\partial x} ; \frac{\partial B_{z}}{\partial y} ; \frac{\partial B_{z}}{\partial z}\right]$. As shown in Fig. 2.4, this phase $\varphi$ is proportional to the pulsed gradient duration $\delta$ and intensity $G$.

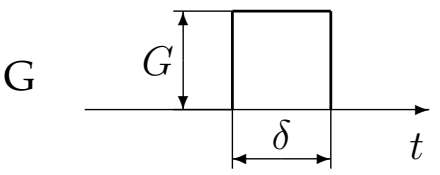

(a)

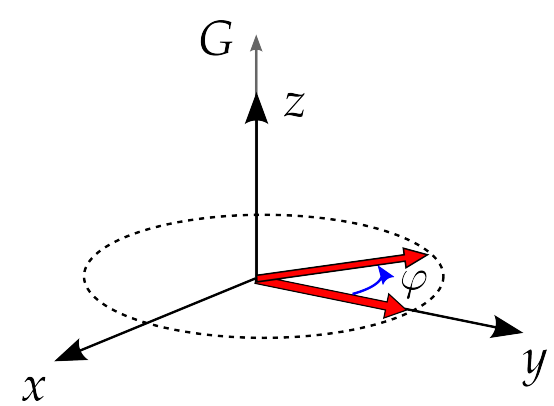

(b)

FIG. 2.4: (a) A pulsed field gradient with duration of $\delta$ and strength of $G$. (b) Transverse magnetization vector driven by pulsed field gradient.

\subsubsection{Free induction decay and spin echo}

Free induction decay. In order to obtain a maximum signal in the transverse plane, a RF pulse rotating $M_{0}$ by $\pi / 2$ is generally applied. The magnetization decay in the transverse plane is then caused by the loss of 
phase coherence in the spin ensemble, which is driven by the molecular interaction or by the magnetic field inhomogeneities. This process can be recorded as a signal called Free Induced Decay (FID). The pulse program and the acquired FID signal is shown in Fig. 2.5. The FID signal decay follows:

$$
M(t)=M_{0} \cdot \exp \left(-\frac{t}{T_{2}^{*}}\right)
$$

where the decay rate of FID is $1 / T_{2}^{*}$.

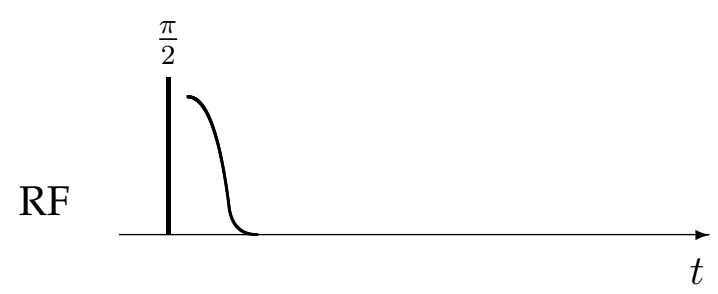

FIG. 2.5: Pulse sequence for detecting FID signal.

Spin echo. The attenuation due to magnetic inhomogeneities in the FID signal can be reversibly compensated by a subsequent $\pi$ RF pulse in the pulse sequence [30]. The pulse program and the acquired signal are shown in Fig. 2.6.

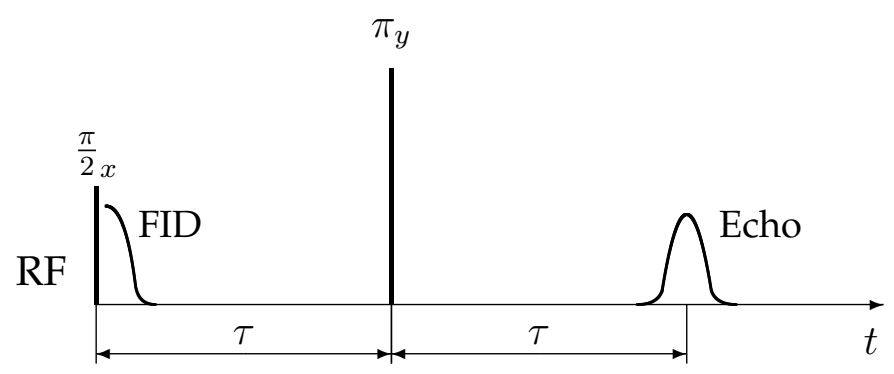

FIG. 2.6: Pulse sequence for detecting spin echo.

After an initial $\pi / 2$ pulse, the magnetization vector is rotated into the transverse plane (Fig. 2.7 (a)) and start dephasing with $1 / T_{2}^{*}$ (Fig. 2.7 (b)). By applying an additional $\pi$ pulse after $\tau$ time, the dephased magnetization components will be rotated to opposite phases (Fig. 2.7 (c)) and start 
rephasing in the transverse plane (Fig. $2.7(\mathrm{~d})$ ). After an equivalent $\tau$ time period, the magnetization can be refocused and a signal can be captured after $2 \tau$ time. This signal is named as spin echo.

During this rephasing process, the field non-uniformities coming from the applied field $B_{0}$ and the local field due to susceptibility differences can be reversed by $180^{\circ}$ since they are time-independent. However, those timedependent fluctuating fields, which is due to the vibration and rotation of neighboring nuclei and is characterized by $T_{2}$, cannot be reversed. The echo is thus weaker than the initial amplitude and its intensity is given as:

$$
M(2 \tau)=M_{0} \cdot \exp \left(-\frac{2 \tau}{T_{2}}\right)
$$

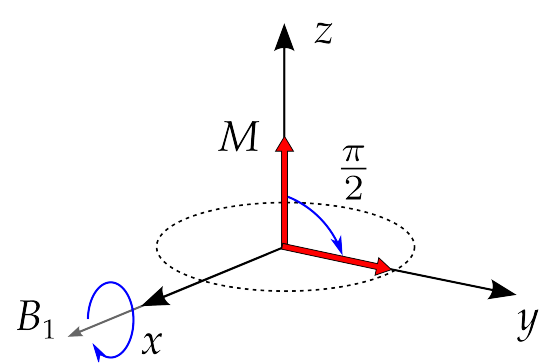

(a)

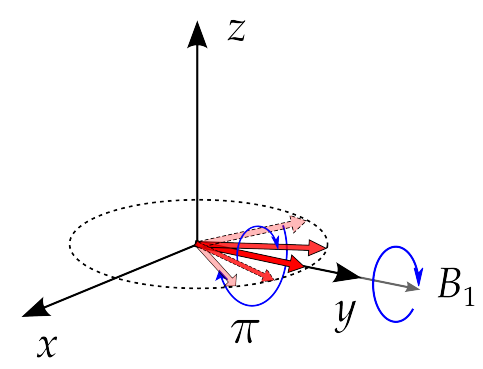

(c)

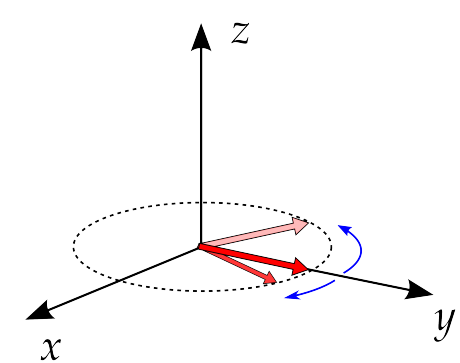

(b)

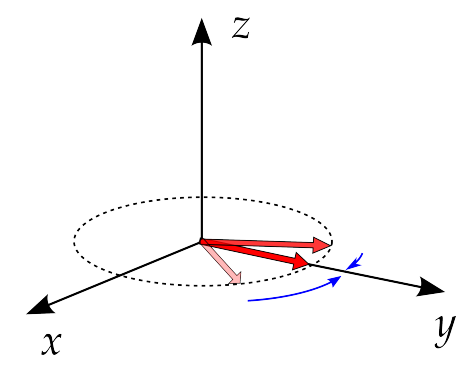

(d)

FIG. 2.7: The process of forming spin echo using magnetization vector.

The invention of this pulse sequence [30] is a huge contribution to NMR 
society since it allows NMR signal surviving in a time scale in the order of $T_{2}$, which is much longer compared to $T_{2}^{*}$ order, thus broadening the NMR observation window. In the following, many techniques for a range of applications, for instance $T_{2}$ measurement, imaging and molecular mobility observation, are inspired from or based on spin echo pulse sequences.

\subsubsection{Relaxometry}

The study of spin systems returning to its thermal equilibrium status is referred to as NMR relaxometry [31]. Two notations, $T_{1}$ and $T_{2}$, are used to describe the process of magnetization evolution along different directions. $T_{1}$, which is so-called spin-lattice or longitudinal relaxation, is a joint property of the spin system and its environment. Its value reflects how efficiently the magnetic energy of the spin system transfer to or from its surroundings. A large $T_{1}$ means weak coupling and slow process for the spin system to achieve thermal equilibrium and thus build up $M_{0}$ along the direction of $B_{0} . T_{2}$ is so-called spin-spin or transverse relaxation. It characterizes the magnetization attenuation of the spin system in the transverse plane, which is caused by the loss of the phase coherence due to local field fluctuations between neighboring spins. Both $T_{1}$ and $T_{2}$ are measured by using the relevant pulse sequences and can often be linked to particular properties of the sample under study, such as fluid presence or material structure.

Inversion recovery pulse sequence can be employed to extract $T_{1}$ relaxation time. Given a $\pi$ RF pulse at the beginning of the pulse sequence shown in Fig. $2.8(\mathrm{a}), M_{z}(0)$ is $-M_{0}$ according to Eq. 2.12. Then after an evolution time $T_{\mathrm{W}}$, the magnetization along $z$ axis is given as:

$$
M_{z}\left(T_{\mathrm{W}}\right)=M_{0} \cdot\left[1-2 \exp \left(-\frac{T_{\mathrm{W}}}{T_{1}}\right)\right]
$$

according to Eq. 2.13. A $\pi / 2$ RF pulse is then utilized to rotate $M_{z}\left(T_{\mathrm{W}}\right)$ to 
$x-y$ plane, the intensity of which is detected by the amplitude of the FID signal.

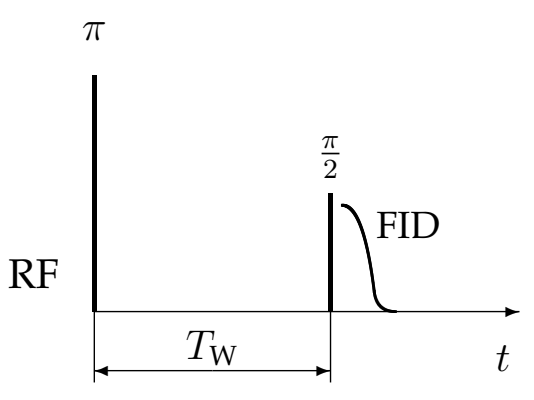

(a)

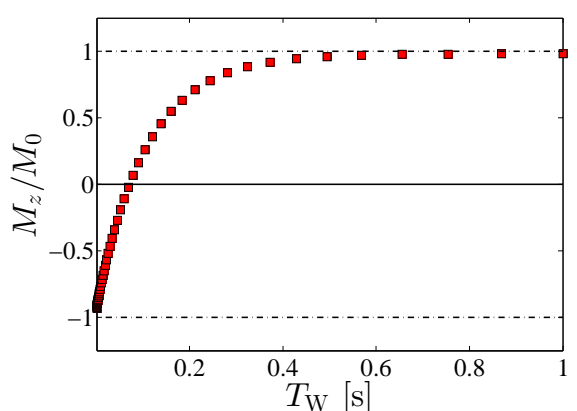

(b)

FIG. 2.8: (a) Inversion recovery pulse sequence for measuring longitudinal relaxation time $T_{1}$. (b) An example of $T_{1}$ measurement of doped water with $T_{1}=105$ ms.

A series of FID signals within variable lengths of $T_{\mathrm{W}}$ can be acquired and $T_{1}$ can be extracted from these data points (An example dataset shown in Fig. 2.8 (b)). Between two successive measurements with different $T_{\mathrm{W}}$ times, a repetition time of $5 T_{1}$ needs to be satisfied in order to recover to thermal equilibrium for the spin system. This condition leads to a timeconsuming acquisition for $T_{1}$ measurement. Rapid encoding methods, for example suggested in [32], can be implemented to record the evolution of $M_{z}$ by using small tip angle pulses. The details and application of this technique will be discussed in Chapter. 5 .

In order to study $T_{2}$ relaxation, a classical pulse sequence, Carr-PurcellMeiboom-Gill (CPMG), can be utilized [33, 34]. It is developed based on spin echo pulse sequence and the pulse programme is shown in Fig. 2.9 (a). By applying $\pi$ pulse in a repeated way, a series of successive spin echoes can be recorded and $T_{2}$ information is stored in the peak decay of the spin echo train. 


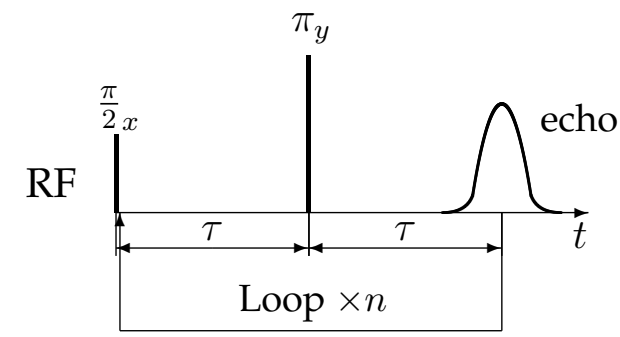

(a)

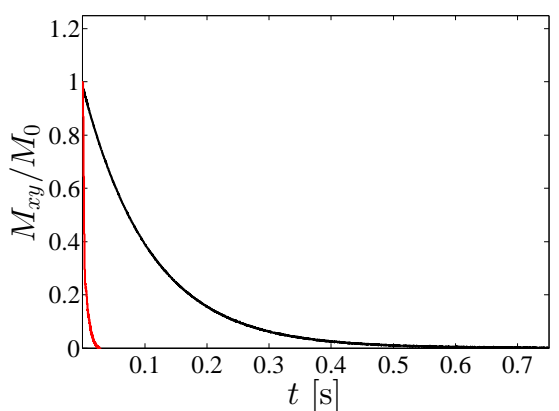

(b)

FIG. 2.9: (a) Pulse sequence for transverse relaxation time $T_{2}$ measurement. (b) An example dataset measured from doped water sample with $T_{2}=100 \mathrm{~ms}$. The black curve is the amplitude attenuation of the acquired echoes from CPMG pulse sequence, which is compared with a reference FID signal with red color here. A relative slow decay rate of black curve as compared to red one indicates that $T_{2} \geqslant T_{2}^{*}$.

The acquired echo intensities can be found in Fig. 2.9 (b) and follow:

$$
M(n \cdot 2 \tau)=M_{0} \cdot \exp \left(-\frac{n \cdot 2 \tau}{T_{2}}\right)
$$

where $n$ is the number of the acquired echoes and $2 \tau$ is denoted to be $T_{\mathrm{E}}$ which is named as the echo spacing of CPMG pulse sequence. The attenuation of CPMG signal, which is characterized by $T_{2}$, arises from spinspin interactions due to the vibration and rotation of neighboring spins. It may be additionally affected by translational diffusion of molecules within the field inhomogeneities. Therefore, an echo spacing $T_{\mathrm{E}}$ as short as NMR probe can accept is normally adopted in CPMG pulse sequence in order to minimize the influence of molecular diffusion in field inhomogeneities.

Compared to FID signal, CPMG echo train allows one to study the relaxation properties in a more approachable way without the influence from the inhomogeneities of magnetic fields (see in Fig. 2.9 (b)). Therefore, it is extensively applied in many fields. It is even robust to extract the transverse 
relaxation properties of the spin system in some extremely inhomogeneous cases by using this technique [35].

\subsubsection{Diffusion}

Diffusion is the molecular displacement of particles (spin-carrying molecules in this thesis). There are two types of diffusion. One is called transport diffusion related to the concentration differences in the neighboring sections. Another one is self diffusion due to the interaction of the molecules undergoing thermal motion. This process is characterized by selfdiffusion coefficient $D$. This characteristic parameter reflects the mobility of molecules and therefore can be used to identify the fluid compositions. We concentrate the study of this thesis on self-diffusion.

The mobility of molecules is expressed by the propagator $P_{s}\left(r, \mid r^{\prime}, t\right)$. This function describes the probability of a particle starting off at $r$ and being located at $r^{\prime}$ after time $t$ later. In a system with a large number of molecules, the averaged propagator theory relates to diffusion process by using diffusion equation (Fick's Second Law) $\partial \overline{P_{s}} / \partial t=D \nabla^{2} \overline{P_{s}}$ and its solution along a certain axis (e.g. z) is given as [36, 37]:

$$
\overline{P_{s}}(z, t)=\frac{1}{\sqrt{4 \pi D t}} \exp \left[-\frac{z^{2}}{4 D t}\right]
$$

with an initial condition of zero displacement for the molecules $\overline{P_{s}}(z, t)=$ $\delta(z)$ and boundary condition $\overline{P_{s}}(z, \Theta) \rightarrow 0$ where $\Theta$ is the diffusion observation time when $z \rightarrow \infty$.

PFG-NMR is a unique technique that measures directly the probability distribution of molecular displacements within the samples, averaged over each individual starting point. As seen from Eq. 2.20, this measured averaged propagator, or its second moment, so-called mean square displacement, scales to diffusion coefficient value of the molecules $D$. Thus, $D$ values can be extracted from PFG-NMR experimental results. 
Two magnetic field gradients with a duration of $\delta$ and separated by a time interval of $\Delta$ are used in order to detect the molecular displacement. $\delta$ is narrow enough here so that the molecular diffusion during this period is negligible [38]. A phase shift of spin-carrying molecules during $\Delta$ can be recorded using this gradient pair. The net phase shift will be zero for the molecules with zero displacement and would be $\gamma \delta G Z$ for the molecule with a displacement of $Z$ along the applied gradient $G$. Therefore, the detected NMR signal as the ensemble average would be the integral over the phases of spin-carrying molecules considering their probability to be located in a certain part of the sample:

$$
M(G, \Delta)=M_{0} \int \overline{P_{s}}(z, t) \exp [i \gamma \delta G Z] d Z
$$

Here, $Z$ is the molecular displacement along the applied gradient direction ( $z$-direction here). Solving Eq. 2.21 using the expression of $\overline{P_{s}}(z, t)$ given in Eq. 2.20, the measured PFG-NMR signal will be:

$$
M(G)=M_{0} \cdot \exp \left[-\gamma^{2} G^{2} \delta^{2} D \Delta\right]
$$

where the exponential term can be corrected to $\exp \left[-\gamma^{2} G^{2} \delta^{2} D(\Delta-\delta / 3)\right]$ considering a finite gradient duration. PFG NMR is generally based on the Pulsed Gradient Spin Echo (PGSE) [39] or the Pulse Gradient STimulated Echo (PGSTE) [40]. An example of measured PGSE signals in three liquids with different self-diffusion coefficients are presented in Fig. 2.10 (b).

There are two advanced NMR methods detecting mean square displacement and further analyzing diffusion coefficient in a one-shot measurement. One is implemented by designing a second order field with linear gradient (rather than constant one) across the sample. The data points with different $G$-values can be acquired in parallel from the segment volume of the sample resolved by imaging method and $D$ can be extracted according to Eq. 2.22 [41]. Another approach is named as Multiple Modulation Multiple Echoes (MMME). By adopting a customized phase cycling, this method selects the 


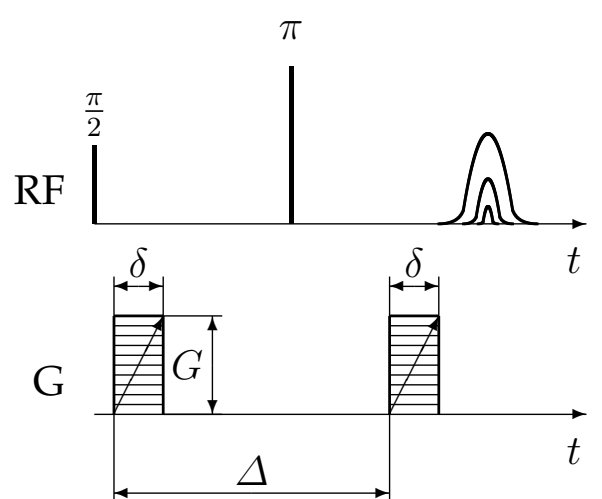

(a)

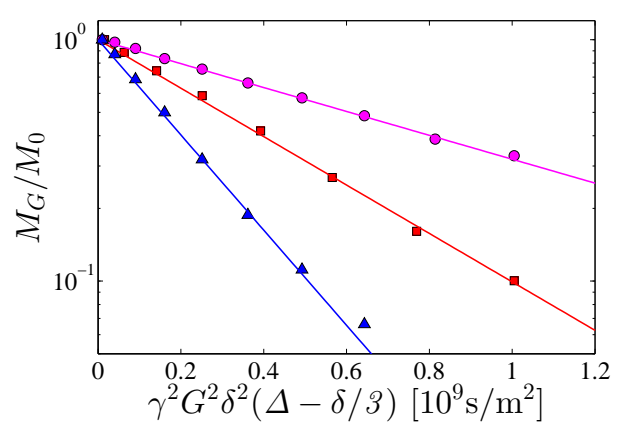

(b)

FIG. 2.10: PGSE pulse sequence (a) and the experimental datasets in ethanol(magenta $\bigcirc$ ), water (red $\square$ ) and acetone (blue $\triangle$ )(b). The straight lines fitted from the datasets stand for the results of $D$ being $1.14 \times 10^{-9} \mathrm{~m}^{2} / \mathrm{s}, 2.31 \times 10^{-9}$ $\mathrm{m}^{2} / \mathrm{s}$ and $4.58 \times 10^{-9} \mathrm{~m}^{2} / \mathrm{s}$ in ethanol, water and acetone at $25^{\circ} \mathrm{C}$, respectively.

independent echo pattern along the acquisition time period. Each echo is sensitive to a certain coherence pathway ${ }^{3}$ in spin dynamics which links to diffusion with a corresponding factor. The modulation of those echoes and the fitting of the echo amplitudes yields diffusion information [44].

\subsubsection{Magnetic Resonance Imaging}

The principle of reconstructing a spin density map using NMR and its Fourier relationship between the spin density and the acquired NMR signal was reported in the early 1970's [45, 46]. This imaging technique was then widely referred to as Magnetic Resonance Imaging (MRI) and is used as a routine approach in health care and in clinical environments. It is furthermore used in many research disciplines, such as medical, biological and material sciences [38, 47, 48].

In order to take a "shoot" of an NMR image, a special designed magnetic

\footnotetext{
${ }^{3} \mathrm{~A}$ coherence pathway is the sequence of coherence orders for the magnetization evolving through the experiment. All NMR measurements start with zero order coherence (z-direction magnetization) and should end with a coherence order of -1 [42, 43].
} 
field, normally linear with a constant gradient, is required and applied across the sample. By using this, the Larmor frequency $\omega$ of the regional spins depends on the local magnetic field strength, therefore the spatial spin density is labelled by $\omega$. To simplify the notation, we address the 1D case in this context. As a result, the local $\omega$ is expressed as:

$$
\omega(z)=\gamma B_{0}+\gamma g z
$$

where $g$ is the magnetic field gradient applied along $z$ direction. Thus, the detected NMR signal from an elementary volume within a slice with a length of $\mathrm{d} z$ would be:

$$
\mathrm{d} M(t) \propto \xi(z) \mathrm{d} z \exp [i \omega(z) t]
$$

where $\xi(z)$ is the spatially distributed spin density and the desired value one wants to obtain from the MRI measurement. By neglecting $\gamma B_{0}$ in Eq. 2.23 (since it only introduces a global but constant phase factor), the acquired signal for an ensemble of elementary volumes with local frequencies can be expressed as:

$$
M(k)=\int \xi(z) e^{i 2 \pi k z} d z
$$

where $k$ is introduced as a wave vector and equals to $\gamma g \delta / 2 \pi$.

Since $k$ is the reciprocal space of $z$ in Eq. 2.25, the proton density $\xi(z)$ can be determined from the measured signal $M(k)$ and is expressed as:

$$
\xi(z)=\int M(k) e^{-i 2 \pi k z} d k
$$

by using well-known Fourier transform (details of data processing will be discussed in Sec. 2.5). As a result, the signal $M(k)$ and proton density $\xi(z)$ are mutually conjugate, which is the fundamental relationship of NMR imaging [49].

To encode the spatial position, MRI is generally based on either fre- 
quency or phase encoded imaging (shown in Fig. 2.11). A magnetic field gradient is applied during signal acquisition for frequency encoded imaging, ensuring spatially distributed resonance frequencies which return the image of the sample upon Fourier transform of the acquired MRI signal. The Field Of View (FOV) for this imaging method is determined to be: $2 \pi / \gamma g \Delta t$, where $g$ is the gradient strength and $\Delta t$ is the time interval between neighboring acquired points (aka dwell time) $[6,38,48]$. In order to obtain an image using the phase encoded MRI method, a set of gradients within variable amplitudes is applied during a fixed time period $\delta$ before acquisition. This returns a set of experiments with varying phases in the acquired MRI signal (only the echo amplitude is needed), which again provides an image after Fourier transform. In this case, the FOV is determined as $m \pi / \gamma g_{\max } \delta$ with $g_{\max }$ being the maximum intensity of the phase gradient. The imaging resolution $\Delta z$ is FOV $/ m$, where $m$ is the number of acquired points in frequency-encoded imaging and the number of gradient steps in phase encoded imaging, respectively $[38,48,50]$.

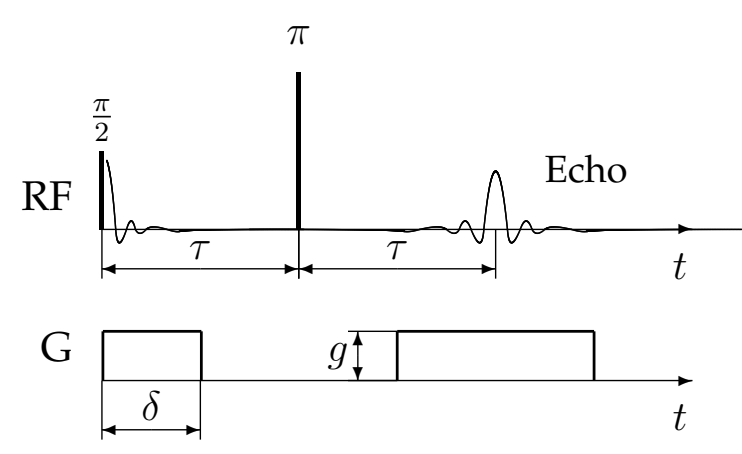

(a)

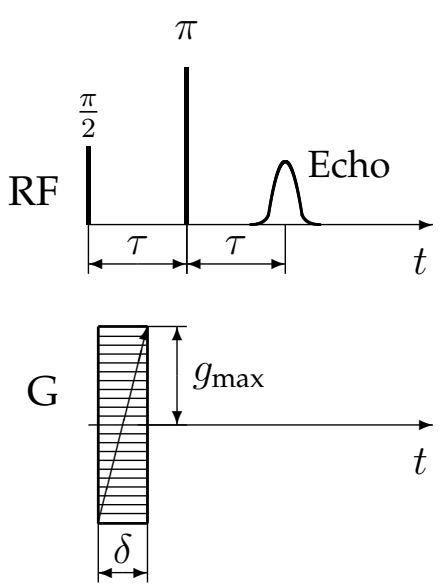

(b)

FIG. 2.11: (a) One-dimensional frequency-encoded imaging pulse sequence. (b) One-dimensional phase-encoded imaging pulse sequence.

Since the spatial information can be encoded in a one-shot signal acquisition under fixed amplitude of the imaging gradient, frequency-encoded 
methods are generally faster as compared to phase-encoded. However, $T_{2}$ effects will irreversibly impact the obtained imaging profile during the acquisition time, leading to the absence of signal with short $T_{2}$ relaxation time. The phase-encoded method on the other hand uses short signal acquisition time at the cost of experimental time. As a result, it minimizes image distortion due to field inhomogeneities from external or induced magnetic fields, and can therefore offer more accurate imaging profiles. Nevertheless, both methods can be employed and combined for high-dimension imaging considering the time efficiency and accuracy $[38,51]$.

\subsection{Multi-dimensional NMR techniques}

Various multi-dimensional NMR methods have emerged allowing for much more detailed insights of the sample properties. According to their pulse sequences and data processing methods (detailed discussed in Section 2.5), the developed multi-dimensional NMR techniques can be classified into three major categories (shown in Tab. 2.1):

- Fourier-Fourier NMR: Oscillatory NMR signals are naturally analyzed using Fourier techniques. In this context, multi-dimensional COrrelation SpectroscopY (COSY) have been widely used to understand the structure of macromolecules, such as protein [52]. Other experiments obtaining oscillatory datasets, such as Velocity EXchange SpectroscopY (VEXSY), Positional EXchange SpectroscopY (POXSY), multi-dimensional propagators, observing the molecules velocities or displacement exchange in a different sub-ensemble, are also parts of this category [53-57]. Furthermore, by observing the molecular motion during variable time intervals in local induced magnetic field exchange experiment, molecular migration and heterogeneity information can be investigated [58-60]. In MR imaging context, frequency and phase encoded methods are normally combined with slice selection. This encoding protocol yields multi-dimensional $k$-space data, 
therefore provides 2D/3D imaging [38, 48]. In order to overcome the limitation of the imaging resolution in $k$-space, emerging multidimensional $q$-space imaging can increase resolution down to $\mu \mathrm{m}$ by utilizing diffusing molecules as imaging agent [61, 62].

- Laplace-Laplace NMR: Signals acquired in relaxometry/diffusometry experiments normally attenuate exponentially and the corresponding data processing approach operates in the space referred to as Laplace domain (detailed given in Section 2.5.2). Plenty of multi-dimensional NMR experiments have been developed based on their 1D counterparts. They can be categorized into two major sorts, correlation and exchange approaches. Multi-dimensional correlation experiments, such as Relaxation-Relaxation Correlation SpectroscopY (RRCOSY), Diffusion-Relaxation Correlation SpectroscopY (DRCOSY), RelaxationInternal gradients Correlation SpectroscopY (RICOSY), DiffusionRelaxation-Internal Gradient Correlation SpectroscopY (DRICOSY) and Diffusion-Diffusion COrrelation SpectroscopY (DDCOSY), couples multiple NMR or NMR-related properties in one experiment, making it easier to understand the dependence and correlation between those parameters [9,63-68] (details shown in Chapter 4). Recently, a novel framework of multi-dimensional RRCOSY technique, denoted as eigenmode correlation experiment, has been developed and applied in porous media to obtain pore morphology and pore surface information (details in Chapter 4). Multi-dimensional exchange experiments, such as Relaxation EXchange SpectroscopY (REXSY) and Diffusion EXchange SpectroscopY (DEXSY) provide insights on the pore structure through the migration of spin population over mixing time $[32,69,70]$.

- Fourier-Laplace NMR: By considering the merits of Fourier and Laplace NMR methods, these two techniques are easily married in the context of spectroscopy/imaging with relaxation/diffusion. For 
instance, Diffusion-Ordered SpectroscopY (DOSY) and RelaxationRelaxation-Ordered SpectroscopY (RROSY) yield diffusion and relaxation correlation maps of molecular compositions within different chemical shift values [32, 71, 72]. Propagator-resolved relaxation and REXSY methods monitor the molecular translational displacements in certain size range in flow experiments and track how far fluid molecules move during the experimental mixing time to estimate critical pore morphologies [73-75]. Lastly, by combing the $k$-space imaging method with relaxation/diffusion and multidimensional RRCOSY/RDCOSY measurements,spatially-resolved relaxation/diffusion profiles and RRCOSY/RDCOSY maps provide local information on pore structure or fluid distribution [76-80].

\subsection{Instrumentation}

The basic components of an NMR analyzer are shown in Fig. 2.12. It normally consists of a probe, a console and a computer. The probe has a magnet for generating $B_{0}$ field for polarization, a RF coil providing $B_{1}$ field to excite spin system and later detecting NMR signal, and optionally gradient layers applying pulsed field gradients for imaging or diffusion measurements. The magnet can be permanent or super-conducting. Depending on the functions and application, the magnet strengths are normally $0.2 \mathrm{~T} \sim$ $3.0 \mathrm{~T}$ for imaging $[6,47,48,88], 1.0 \mathrm{~T} \sim 21.0 \mathrm{~T}$ for spectroscopy $[89,90]$ and $0.012 \mathrm{~T} \sim 9.4 \mathrm{~T}$ for relaxometry $[7,16,69]$. There exists many options for RF coils depending on the measuring patterns, such as solenoid, bird cage, saddle and surface coils, etc. The RF coil is tuned to the required NMR frequency and matched to $50 \Omega$ impedance, in order to deliver the maximum RF power from the transmitter to the probe and the maximum signal received from the probe to the receiver. The tip angle $\alpha$ can be adjusted by the strength of $B_{1}$ or the duration $t_{\mathrm{p}}$ according to Fig. 2.3. In this thesis, we adapt $\alpha$ by modifying the strength of $B_{1}$. This is achieved by controlling 


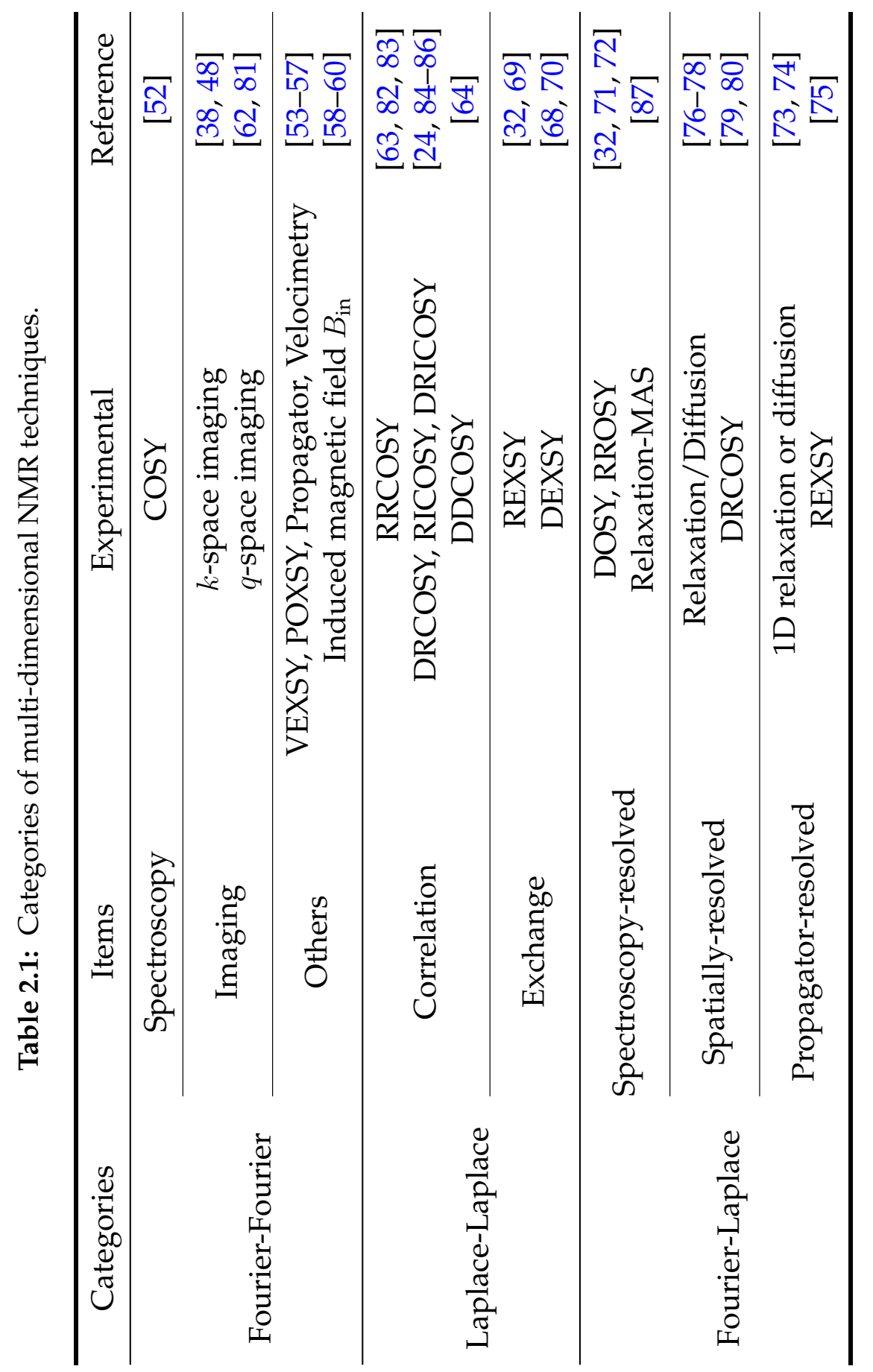


the current strength in RF coil delivered by the RF power amplifier of the spectrometer ${ }^{4}$.

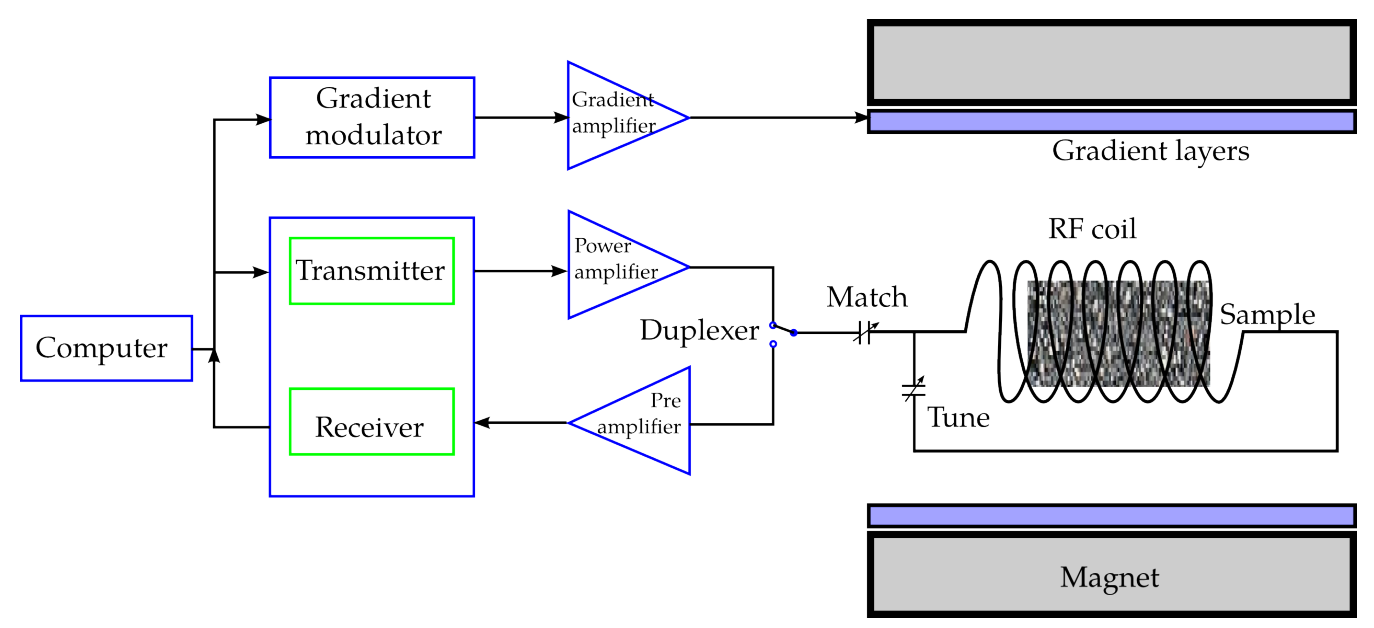

FIG. 2.12: Schematic of an NMR analyzer.

The console or spectrometer which is connected to the probe is operated by the computer. Controlled by the pulse program from the computer, transmitter sends the excitation impulses generated by a synthesizer inside. After that, these pulses are amplified to a required excitation power level by the power amplifier. Then the energy is transmitted to the RF coil to irradiate the sample via a transmit-receiver switch, the so-called duplexer. Through another amplifier to magnify the induced signal from the RF coil to a receivable level and a Analogue-to-Digital Converter (ADC), the receiver will obtain a quadrature signal with both real and imaginary parts.

The gradient modulator provides the time-dependent currents and drives the gradient layers to generate additional magnetic fields in the sensitive volume. Those fields with well-defined gradients can be widely utilized, such as to optimize the homogeneity of the major static field $B_{0}$, to encode the position information in imaging experiments or/and observe the molecular motion process in NMR diffusion measurements.

\footnotetext{
${ }^{4}$ Denoted in deciBels $(\mathrm{dB})$ unit, the difference of $6 \mathrm{~dB}$ would be a ratio of $10^{-0.1 \times 6} \approx 0.25$ for power attenuation, which approximately halves the intensity of $B_{1}$. This relationship is normally kept in $\pi / 2$ and $\pi$ pulses in this thesis.
} 


\subsection{Data processing}

There are mainly two types of data patterns exhibiting in NMR experiments: oscillatory and exponential. Therefore, the corresponding methods treating each type of them and extracting the involved NMR properties are discussed in this section.

\subsubsection{Fourier Transform (FT)}

A generalized expression of oscillatory signals from NMR spectroscopy or imaging experiments can be given in Eq. 2.27 as:

$$
f(t)=\frac{1}{\sqrt{2 \pi}} \int_{-\infty}^{+\infty} F(\omega) \exp (i \omega t) d \omega
$$

where $F(\omega)$ is the distribution function in the reciprocal space. Depending on the cases, it could be specified to NMR spectra or spatial spin density distributions (as discussed in Eq. 2.25 and 2.26). In order to obtain $F(\omega)$, the "time-domain" signal $f(t)$ will be transformed into "frequency-domain" by using Fourier Transform (FT):

$$
F(\omega)=\frac{1}{\sqrt{2 \pi}} \int_{-\infty}^{+\infty} f(t) \exp (-i \omega t) d t
$$

Here, the terms of "time-domain" and "frequency-domain" have wider meaning. Taking NMR spectroscopy in chemical analysis as an example, the acquired FID signal is a "time-domain" dataset and the obtained nuclei spectrum can be considered as the data along "frequency-domain". A similar case of such a data pair can be found in an imaging dataset (shown in Fig. 2.13). As discussed in Eq. 2.25 and 2.26, the time-domain data refers to $k$-space data and it can be transformed into frequency-domain which relates to real space. 


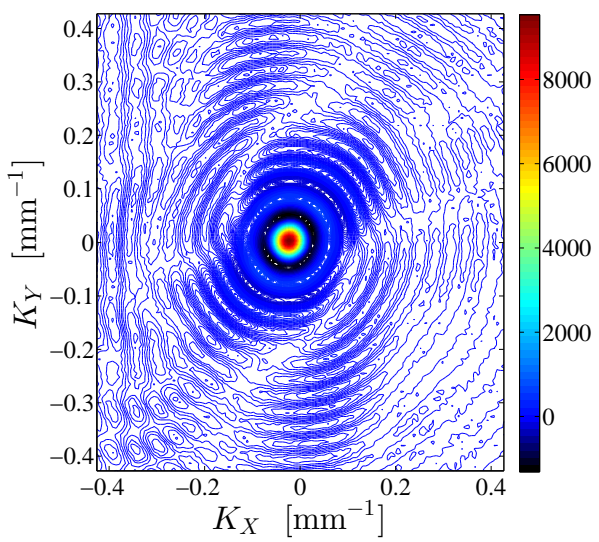

(a)

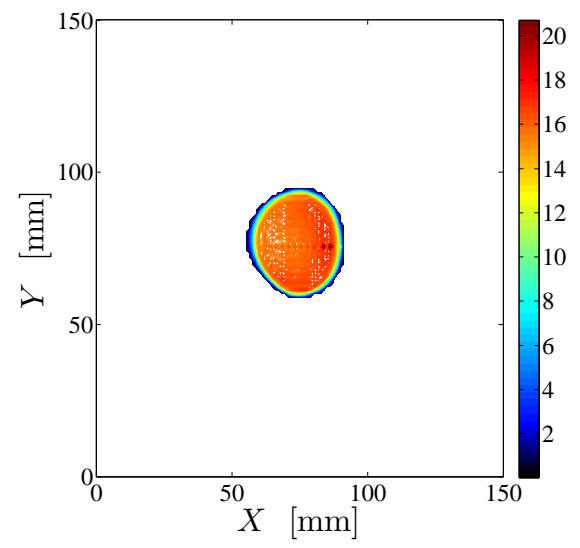

(b)

FIG. 2.13: A two-dimensional $k$-space data measured from phantom (a) and its FT imaging result (b). Here $k$-space signal and its transformed image can be considered as "time-domain" and "frequency-domain" datasets, respectively.

\subsubsection{Inverse Laplace Transform (ILT)}

In relaxometry or diffusometry measurements, the acquired signals normally follow multi-exponential decay and their expression can be generalized as:

$$
g(t)=\int \exp (-s t) f(s) d s=\int K(t, s) f(s) d s
$$

where $g(t)$ is the measured NMR signal, $f(s)$ represents the relaxometry or diffusometry information, depending on the case, $s$ can be self-diffusion coefficient $D$ or relaxation rates $1 / T_{1,2} . K(t, s)$ is the kernel matrix with an exponential form in the case of $1 \mathrm{D}$ relaxation/diffusion experiments or multi-dimensional NMR experiments. This equation thus follows the form of Laplace Transform (LT).

In order to obtain the relaxometry or diffusometry properties of the materials discussed in Sec. 2.2.3 and Sec. 2.2.4, the data processing in this problem consists of finding the function $f(s)$ given a continuous kernel 
function $K(t, s)$ and a function $g(t)$. This process is known as Inverse Laplace Transform (ILT) and falls into the context of a well-known "Fredholm integrals of the first kind", which is typically ill-posed ${ }^{5}$.

To solve this problem in a stabilized way, it is typically more convenient to decompose ILT problem into transforms consisting of linear functions. A general approach called optimization is applied in this context in the first step:

$$
\hat{f}=\underset{f \geqslant 0}{\operatorname{argmin}}\|g-K f\|^{2}
$$

where $\|\cdots\|$ is the $l_{2}$ (Euclidean) norm. Many methods were developed to solve this optimization problem, such as NNLS [91], Maximum entropy [92] and Tikhonov regularization [93]. In this thesis, we focus on using the last one. Tikhonov regularization offers a more refined resolution by attaching an extra smooth term. The expression is given as:

$$
\hat{f}=\underset{f \geqslant 0}{\operatorname{argmin}}\|g-K f\|^{2}+\lambda\|f\|^{2}
$$

where $\lambda$ is smoothing parameter applied to the final solution. The smoothing degree is controlled by tuning $\lambda$ that acts as a weight on the significant singular values of kernel matrix $K$. Therefore, the perturbation due to smaller singular values in the final solution can be suppressed. The choice of using an optimized $\lambda$ is critical. An overestimated $\lambda$ will introduce too much interference to the final solution whereas an underestimated one is inappropriate to stabilize the discrete solution. Thus, many methods, such as L-curve, S-curve, can be utilized to determine an optimized $\lambda$ over a pre-defined range of $\lambda$ values. After fixing the $\lambda$ value used in Eq. 2.31, the

\footnotetext{
${ }^{5}$ The problem $\mathcal{R}$ of determining a function $f(s) \in \mathcal{F}$ through a given function $g(t) \in \mathcal{G}$, $f(s)=R[s, g(t)]$ (where $\mathcal{F}$ and $\mathcal{G}$ are function spaces) is ill-posed if any of the following conditions can not be satisfied:

A. For every function $g(t) \in \mathcal{G}$, there exists a solution $f(s)$ of the problem.

B. The solution $f(s)$ is unique for a given $g(t)$.

C. The dependence of the solution $f(s)$ on $g(t)$ is continuous in the space of $\mathcal{F}$ and $\mathcal{G}$.
} 
distribution function $f$ can be obtained using Butler-Reeds-Dawson (BRD) method [94].

The steps of obtaining $\hat{f}$ are achieved by solving $\hat{K}^{\prime}\left(\hat{K} \cdot \hat{K}^{\prime}+\lambda \cdot \mathrm{I}\right)^{-1} \hat{g}$ according to Eq. 2.31:

1: After choosing $\lambda$, a vector $c=\left(\hat{K} \cdot \hat{K}^{\prime}+\lambda \cdot \mathrm{I}\right)^{-1} \hat{g}$ can be calculated first.

2: Using this $c$ vector, a temporary solution $\tilde{f}$ can be obtained. There are possibilities of existing negative elements in vector $\tilde{f}$, which is meaningless in physical concept. Therefore, a non-negative restraint needs to be employed in next step.

3: Locating the negative elements in $\tilde{f}$ and zero-filling the corresponding elements in the matrix $\hat{K}^{\prime}$. An updated vector $c^{\prime}$ can be calculated using the new matrix $\hat{K}^{\prime}$.

4: Iterate step 3 until the difference between neighboring obtained $c$ vectors is smaller than a pre-set threshold value. Then the final convergence solution $\hat{f}=\hat{K}^{\prime}\left(\hat{K} \cdot \hat{K}^{\prime}+\lambda \cdot \mathrm{I}\right)^{-1} \hat{g}$.

For two-dimensional Laplace experiments, the measured $g$ would be a matrix with large size corresponding to kernel matrices $K_{1}$ and $K_{2}$. In order to obtain $\hat{f}$ in an appropriate way, a compressed approach is implemented on $g$ by using truncated kernel matrices $\tilde{K}_{1}$ and $\tilde{K}_{2}$. Considering the importance of the information contained in kernel matrices, one can truncate them using Singular Value Decomposition (SVD) method. These under-sized kernel matrices $\tilde{K}_{1}$ and $\tilde{K}_{2}$ are employed to compress the acquired signal $g$ to be $\tilde{g}$, the size of which depends on the number of truncated singular values. Therefore, $\tilde{g}$ becomes a matrix with much smaller size as compared to $g$ and this multi-dimensional data set can be rearranged to an $1 \mathrm{D}$ case. Here, $\hat{K}^{\prime}=\tilde{K}_{1} \otimes \tilde{K}_{2}$, where $\otimes$ denotes the tensor product [84, 95-99]. An example of 2D time-domain data from doped water sample and its ILT result was shown in Fig. 2.14. 


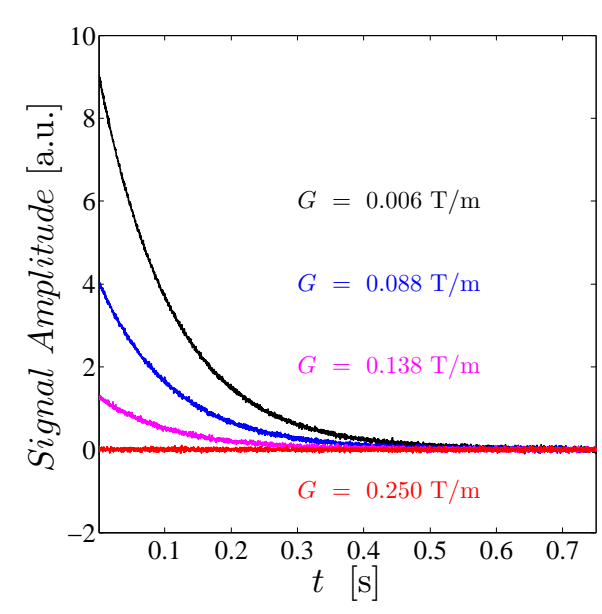

(a)

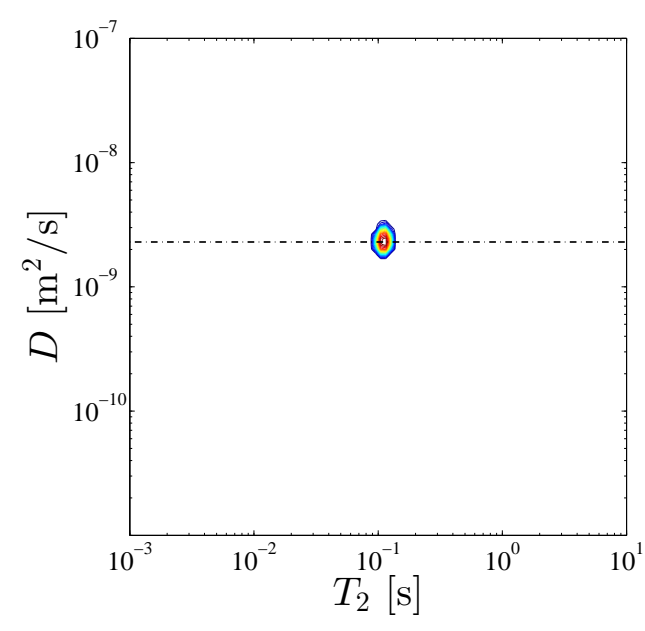

(b)

FIG. 2.14: (a) $D-T_{2}$ experimental data from doped water sample. A combination pulse sequence of PGSTE with CPMG is used to acquire the data. The data size is $35 \times 3000$. Gradient $G$ linearly ramps up from $0.006 \mathrm{~T} / \mathrm{m}$ to $0.25 \mathrm{~T} / \mathrm{m}$ in 40 steps. Echo spacing $T_{\mathrm{E}}$ is $200 \mu \mathrm{s}$, and echo number is 3000 . Only four data traces are shown here. (b) The correlation function $F\left(D, T_{2}\right)$ distribution. The horizontal dashed line represents a self-diffusion coefficient value of $2.3 \times 10^{-9} \mathrm{~m}^{2} / \mathrm{s}$ at $25^{\circ} \mathrm{C}$.

The limitation, resolution and uncertainty of the results yielded from ILT algorithm were also discussed [100-105] and new methods in the frame of ILT [106-113] were further developed to suite some particular cases. 


\section{Chapter 3}

\section{Rock Core Analysis using NMR}

NMR techniques specialized for petrophysical application are reviewed in this chapter. Particular issues of performing NMR measurements in rock analysis are addressed first. Many petrophyscial characteristics, such as porosity, pore length, permeability, tortuosity and a dynamic flooding process can be easily obtained or well described by using this non-invasive technique.

\subsection{Particular consideration for NMR in rocks}

\subsubsection{Nuclear species}

There are plenty of nuclei investigated in NMR rock core analysis. As a major candidate, ${ }^{1} \mathrm{H}$ is dominantly studied in rock core analysis since hydrogen-rich fluids (water and hydrocarbon) are the most interesting species in geological and geophysical contexts. Meanwhile, other nuclei such as ${ }^{13} \mathrm{C}[114,115],{ }^{23} \mathrm{Na}[8,116,117],{ }^{7} \mathrm{Li}$ and ${ }^{19} \mathrm{~F}[118]$, and hyperpolarized noble gas ${ }^{129} \mathrm{Xe}$ [119-121], are also utilized to provide distinctive information, such as organic matter content, salinity and long-distance heterogeneity. Because these investigations are based on "exotic" nuclei, these methods can be considered as unconventional NMR protocols in rock analysis. The details for these nucleus (including hydrogen) with their 
Larmor frequencies at $1 \mathrm{~T}$, natural abundance and spin number are given in Table. 3.1.

Table 3.1: Properties of nuclear species used in NMR rock core analysis. Data adapted from $[4,48,122]$.

\begin{tabular}{lccc}
\hline Species & $\begin{array}{c}\text { Resonance } \\
\text { frequency for } 1 \mathrm{~T} \\
\text { field (MHz) }\end{array}$ & $\begin{array}{c}\text { Natural } \\
\text { abundance } \\
(\%)\end{array}$ & $\begin{array}{c}\text { Spin number } \\
\text { I }\end{array}$ \\
\hline${ }^{1} \mathrm{H}$ & 42.576 & 99.984 & $1 / 2$ \\
${ }^{7} \mathrm{Li}$ & 16.546 & 92.410 & $3 / 2$ \\
${ }^{13} \mathrm{C}$ & 10.705 & 1.108 & $1 / 2$ \\
${ }^{19} \mathrm{~F}$ & 40.055 & 100.000 & $1 / 2$ \\
${ }^{23} \mathrm{Na}$ & 11.262 & 100.000 & $3 / 2$ \\
${ }^{129} \mathrm{Xe}$ & 11.777 & 26.440 & $1 / 2$ \\
\hline
\end{tabular}

\subsubsection{Magnet strength}

The NMR magnets provide the $B_{0}$ field and its strength determines signal intensity in NMR analysis according to Eq. 2.9. However, rock samples are complex regarding to solid purity, compositions, pore network structures and saturating fluids. Such complexity will influence the actual local magnetic field strength in the sample when the rock is exposed to the magnetic field $B_{0}$. Therefore, it is essential to choose a proper magnet for NMR petrophysical application.

Rock core analysis using NMR can be carried out for instance at high magnetic field strength which are typical for clinical purpose (magnet strength $\geqslant 1.5 \mathrm{~T}$ and corresponding to ${ }^{1} \mathrm{H}$ Larmor frequency $\geqslant 64 \mathrm{MHz}$ ). In this case, many imaging techniques developed for studying biological tissue can be "translated" to the imaging of rock cores [123-125]. However, extra consideration needs to be taken into account before performing MRI on rock cores at such field strengths because image results may carry artefacts due to the susceptibility contrasts between the solid grains and 
pore filling fluids. The existence of paramagnetic impurities in rocks will introduce extra relaxation effects and thus deteriorate the imaging results. Nevertheless, particular imaging protocols, such as SPRITE [126] and EPI [127], are still successfully implemented and supply fluid presence and structural information of rock cores.

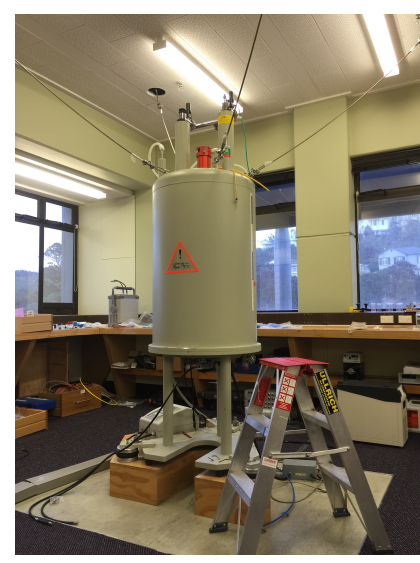

(a) High-field

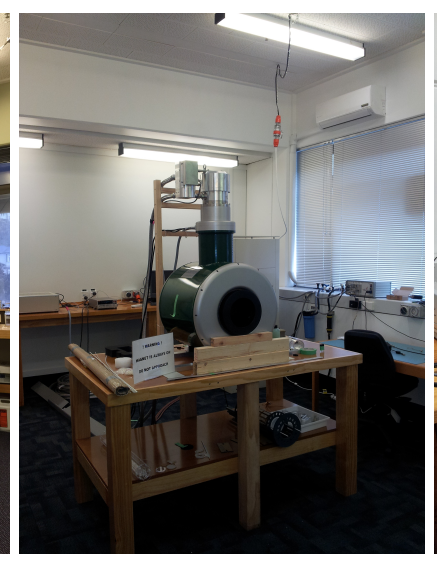

(b) Interediate-field

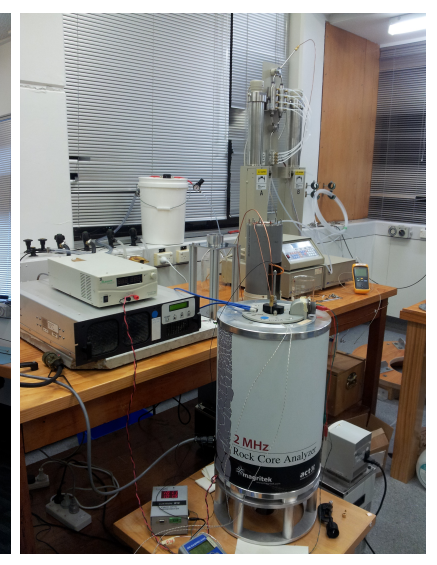

(c) Low-field

FIG. 3.1: NMR instruments that can be utilized for rock core analysis in Victoria University of Wellington. The corresponding ${ }^{1} \mathrm{H}$ frequencies are (a) $400 \mathrm{MHz}$, (b) $64 \mathrm{MHz}$ and (c) $2 \mathrm{MHz}$, respectively.

With the extension and development of NMR techniques, low-field NMR obtains wide acceptance in petropysical applications since it appeases the interaction effect between rock cores and magnets. In this context, there are mainly two ways to carry out NMR measurements regarding their magnet layouts. The first one is similar to the typical high-field magnet construction (outside-in, a magnet bore with sample inside) and another one uses unilateral magnetic field, which is so-called inside-out. The measured sample in the later pattern is not restricted by the available volume of the magnet, therefore, obtaining more and more interests from industry fields [16, 26, 35, 128-131]. In laboratory measurements, the first layout normally adopts a magnet field strength of $0.05 \mathrm{~T}$ (corresponding to $2 \mathrm{MHz}{ }^{1} \mathrm{H}$ Larmor frequency, as seen in Fig. 3.1 (c)) since it is close to 
the field strength for NMR well-logging used in the oils field down-hole measurements [128, 129, 132, 133]. As a result, experimental results from laboratory can be used to calibrate the NMR well-logging data [89, 134, 135]. These measurements mainly rely on some robust methods, such as relaxometry and diffusometry techniques. Imaging methods are still possible, but mainly focused on 1D considering Signal-to-Noise ratios (SNR) of the dataset and the measurement efficiency.

Recently, an intermediate-field NMR instrument (magnet strength $\approx 0.3 \mathrm{~T}$ and corresponding to $12.5 \mathrm{MHz}{ }^{1} \mathrm{H}$ Larmor frequency) is recommended and believed to be a promising choice for NMR core analysis, since it compromises between the requirements of relaxometry/diffusometry and MRI protocols in rock cores $[7,8,136]$.

It is worth mentioning that the huge "magnet"-earth (the field strength $18 \sim 65 \mu \mathrm{T}$, corresponding to $0.8 \sim 2.8 \mathrm{kHz}^{1} \mathrm{H}$ Larmor frequency), is used in Magnetic Resonance Sounding (MRS) for exploiting hydraulic formations. Commonly, $T_{2}^{*}$ is measured in this context. More advanced methods and instruments for acquiring $T_{2}$ and $T_{1}$ curves are developed recently [137143].

\subsubsection{Internal magnetic field $B_{\text {in }}$}

When performing NMR analysis on fluid-saturated rock cores, there will inevitably be local spatial variations in the magnetic field due to the susceptibility contrast between sediment grains and pore filling fluid. This spatial variation is named as internal magnetic field $B_{i n}[5,144]$.

In order to understand the spatial variation and dependence of this induced magnetic field in pore space, rock core can be "digitized" to individual sediment grains. Considering a spherical grain with susceptibility of $\chi_{g}$ in the fluid with susceptibility of $\chi_{f}$, the magnetic dipole moment of this grain at the static magnetic field $B_{0}$ can be approximately expressed as: 


$$
\mathbf{m}=\frac{4}{3} \pi R^{3} \delta \chi B_{0} \hat{\mathbf{k}}
$$

where $\delta \chi=\left|\chi_{g}-\chi_{f}\right|$, denoted as the susceptibility contrast between the grain and the fluid. $R$ is the radius of this grain.

The induced magnetic field according to this magnetic dipole moment $\mathbf{m}$ at a distance of $\mathbf{r}$ can be calculated as:

$$
\mathbf{B}_{i n}(\mathbf{r})=\frac{3(\mathbf{m} \cdot \mathbf{r}) \mathbf{r}-|\mathbf{r}|^{2} \mathbf{m}}{|\mathbf{r}|^{5}}
$$

Since the magnitude of this locally induced field, $\left|\mathbf{B}_{i n}\right|$, are far smaller than $B_{0}$, in the NMR context we only consider the component of $\mathbf{B}_{i n}$ along $B_{0}$, and referred to as $B_{i n}$.

For the case of dense packed grains, the induced magnetic field $B_{i n}$ in the particular position in the pore space can be computed as a superposition of the induced magnetic field from each individual grain (see in Fig. 3.2). Therefore, it can be expressed as:

$$
B_{i n}=\sum_{k} B_{i n}^{k}\left(r_{k}\right)
$$

where $r_{k}$ is the distance between the $k$-th grain to the calculated position. Therefore, as shown in Fig. 3.2, the intensity of internal magnetic field $B_{i n}$ is relatively higher in the area close to the corners of pore voids as compared to the centre of the pore space.

People took a lot of efforts to investigate the dependence of $B_{\text {in }}$ during NMR experiments [85, 86, 145-147]. These computational or experimental work suggested two major features of $B_{i n}$. Firstly, $B_{i n}$ is proportional to the applied magnetic field $B_{0}$. This is easy to understand from Eq. 3.1 and Eq. 3.2. As a result, this intrinsic field will affect interpreting NMR results in rocks particularly at high field strength magnet, such as spectroscopy [148], transverse relaxation time/pore geometry [149-151] and imaging [48], and needs to be suppressed by novel pulse sequence and strategies 


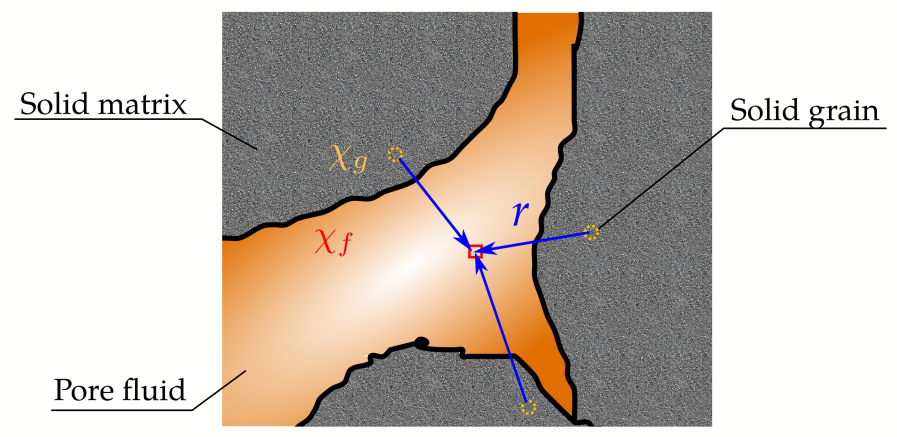

FIG. 3.2: Geometry of local magnetic fields in rock pore space. Note that the map color close to the middle section is shallower, which represents a relatively lower intensity of internal magnetic field $B_{i n}$ compared to the area close to the corners of pore voids.

$[148,152-156]$. Secondly, the variation of $B_{\text {in }}$ occurs primarily over the pore length scale. Therefore, this inherent field can be employed as a "fingerprint" to determine the pore length scales $[157,158]$ and to characterize the heterogeneity of the structural compositions $[159,160]$.

\subsection{Porosity $\phi$}

Porosity $\phi$, or pore volume fraction, is an important parameter determining the fluid reserves in reservoirs [161]. It is defined by the volume ratio of the void space to the rock sample, therefore, is always expressed in percentage. According to Eq. 2.9, NMR signal arises from the fluid spins bearing in the open or connected pore voids and this phenomenon can be straightforwardly utilized to determine porosity. As a result, the porosity obtained from NMR technique is called "effective porosity" rather than "total porosity" [4] as shown in Fig. 3.3 (a). In the mean time, a calibration sample needs to be used to quantify the signal amplitude in the unit volume. 
The porosity calculation of rock cores from NMR signals is described as:

$$
\phi=\frac{S_{0}(\text { rock })}{S_{0}(\text { calib }) \cdot \frac{N S(\text { rock })}{N S(\text { calib })} \cdot \frac{V(\text { rock })}{V(\text { calib })}}
$$

where $S_{0}$ represents the initial amplitude of NMR signal, which links to the net magnetization $M_{0}$, and therefore is proportional to the amount of fluid. $N S$ is Number of Scan during the experiments and $V$ stands for the sample volume. An example of obtained NMR porosities comparing to weighing porosities in rock cores is shown in Fig. 3.3 (b). The correlating dots are mainly located along the diagonal line within the dashed lines representing a range of \pm 1 porosity unit (p.u.), which gives an indication of NMR reliability in rock core porosity measurements.

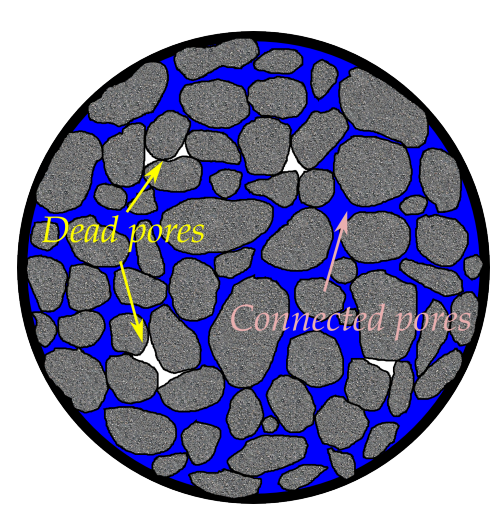

(a)

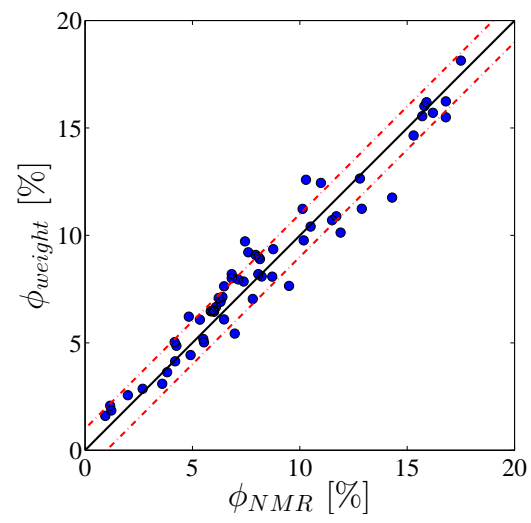

(b)

FIG. 3.3: (a) Schematic of pore network diagram. Because of the NMR principle, the "dead" pores isolated from the saturating fluid contribute no NMR signals. (b) Comparison of weighing porosity with NMR porosity in 1.5 inch rock cores saturated with distilled water. The dashed lines indicate a range of \pm 1 porosity unit (p.u.). 


\subsection{Pore length $a$}

Pore length is a critical structural parameter of porous rocks. The theory of using NMR to determine this dimension mainly relies on Bloch-Torrey equation, which describes the liquid molecules diffusion in pore space and the interaction in pore surface area [162]. A customary solution of the BlochTorrey equation is suggested in terms of a serial expansion of eigenmodes $[163,164]$. These can be categorized into ground and high excited modes according to their spatial dependence, which are sensitive to the relaxation of pore fluid spins at the pore surface and their diffusion in the pore space, respectively. Since both mechanisms relate to pore morphology, according methods based on these two eigenmodes are developed to determine the pore length $a$ independently.

\subsubsection{Surface relaxation}

Because of the presence of paramagnetic impurities, the spin relaxation is accelerated near the pore walls, which is referred to as surface relaxation. This concept actually corresponds to the ground eigenmode (with an index of $n=0$ ) of relaxation in the diffusion equation [163], which characterizes the interaction of saturating fluid spins in the vicinity of the pore surface (as shown in Fig. 3.4 (a)). The decay rates of the measured signals according to surface relaxation will be given as [165]:

$$
\frac{1}{T_{1,2}} \approx \rho_{1,2} \cdot \frac{S}{V}
$$

where $S / V$ is the surface-to-volume ratio which describes the pore size $a$ assuming a certain pore shape. $\rho$ is the surface relaxivity at the solid/fluid interface. Normally $\rho$ can be obtained by matching 1D $T_{1}$ (or $T_{2}$ ) distribution with the pore length scales measured from Mercury injection, timedependent diffusion coefficients or BET gas absorption [166-170]. Other advanced techniques determining $\rho$ will be discussed in details in Sec. 4.3. 
Nevertheless, the signal attenuation ascribed to surface relaxation can be acquired using the pulse sequences introduced in Sec. 2.2.3.

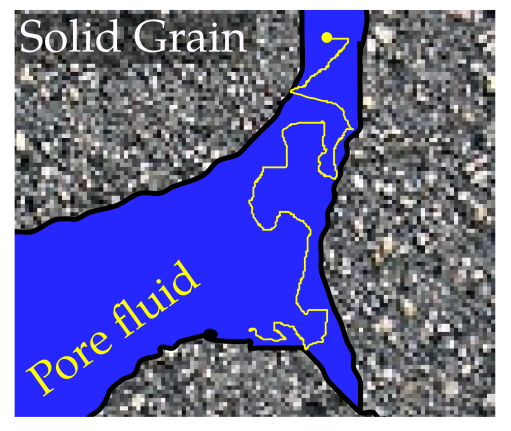

(a)

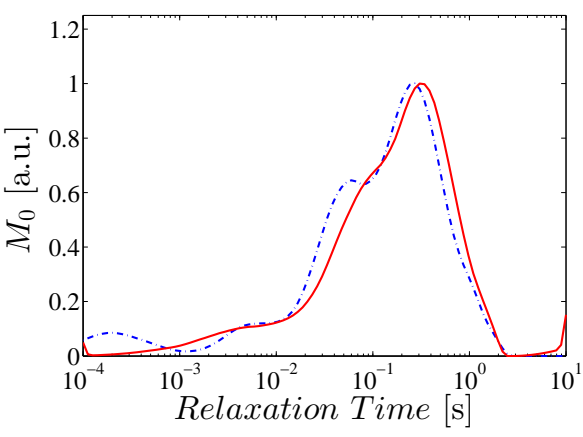

(b)

FIG. 3.4: (a) The notation of fluid spins experiencing surface relaxation close to pore wall area. (b) An example of $T_{1}$ (red solid) and $T_{2}$ (blue dashed) relaxation time distributions in limestone at $2 \mathrm{MHz}$ NMR measurement. The comparable distributions agree with Eq. 3.5.

Note that the measured magnetization normally exhibits multi-exponential decay in rock samples since there always exists broaden pore size distribution ranging over several length orders. As a result, the dataset is usually processed using ILT algorithm as discussed in Sec. 2.5.2. Afterwards, relaxation time distributions can be obtained in laboratory or in-situ well-logging measurements (as seen in Fig. 3.4 (b)) and these results will indicate pore size distributions of rocks $[5,13,14]$.

Compared to $T_{1}$ acquisition, $T_{2}$ measurements in rock cores are more rapid, however, they are also more sensitive to the induced internal magnetic fields $B_{i n}$, especially at high $B_{0}$ field strength as indicated in Section 3.1.3. An efficient calibration strategy for obtaining reliable $T_{2}$ distributions at high-field NMR rock measurements is suggested in [150]. 


\subsubsection{High relaxation eigenmode}

The pore length distributions from the surface relaxation measurements are always convoluted with surface relaxivities $\rho$, leading to be an indirect protocol for determining pore size. Since higher relaxation eigenmodes (with mode index $n>0$ ) are ascribed to the diffusion process of spincarrying molecules in pore space, it provides a straightforward access to pore length $a$. However, the signal intensity in relaxation measurement is dominated by the ground eigenmode and the contribution from high eigenmodes are too weak to be detected. As a result, it is essential to enhance the weights of high eigenmodes before extracting the pore length a.

One relevant technique was developed depending on the inherent magnetic fields $B_{i n}$ in pore space $[157,158]$. As concluded in Sec. 3.1.3, the spatial variation of $B_{i n}$ acts primarily over the pore length. Within a welldefined encoding period, the magnetization profile in pore space can be modulated similar to $B_{i n}$. Furthermore, the evolution of this modulated magnetization maybe recorded and the contributions from high eigenmodes during this procedure largely enhanced. Consequently, the decay rates from the contribution of high mode can be transformed to pore length scales. A more detailed explanation of this technique will be discussed in Sec. 4.1.1 in Chapter. 4.

\subsubsection{Other methods}

There are some other NMR methods yielding pore length information of rock cores. By observing the molecular mobility in confining pore space, the restricted diffusion at variable observation time periods delivers a mean surface-volume ratio [166, 171]. Another method reconstructs pore length scales from grain size distribution obtained from Bayesian magnetic resonance approach [169]. Furthermore, NMR cryoporometry has ability to obtain the pore size ranging from $\mathrm{nm}$ to $\mu \mathrm{m}$ via observing the depressed 
melting point of liquid confined in pore space [172-174].

\subsection{Heterogeneity}

Many types of heterogeneities are adopted to describe the variation of petrophysical property in different scales, such as microscopic (pore/grainscale) heterogeneity, mesoscopic (well-scale) heterogeneity and macroscopic (inter well-scale) heterogeneity [1]. In this thesis, we concentrate on the microscopic heterogeneity.

Microscopic heterogeneity provides the clues about the processes of sediments deposition and subsequent compaction, cementation and/or dissolution during the geological evolution period. Variations in this scale largely influences some petrophysical characteristics, such as permeable and mechanical behaviors. Two general features, pore-network structure and mineral compositions, are normally associated with microscopic heterogeneity.

Direct NMR methods to evaluate the structural and compositional heterogeneity of rocks are by labelling the broadness of pore length and grain size distributions [169]. The wider of these scales, the more heterogeneous pore system or solid matrix would be. Furthermore, multi-dimensional RRCOSY and RREXSY methods can also be utilized in a higher domain, to obtain the pore-network hierarchy and the pore-to-pore connectivity in different regimes $[63,69]$. Moreover, solid composition variation over certain length scales can be estimated by correlating longitudinal relaxation distribution with susceptibility contrast spectrum in 2D correlation maps [160].

Meanwhile, the heterogeneity information can be gained from various MRI techniques by visualizing the spatial presence of fluid and solid components in the MR images [50,78, 123-126, 175]. An example of MR image in a heterogeneous rock sample is given in Fig. 3.5 where the local heterogeneity details can easily be recognized. 


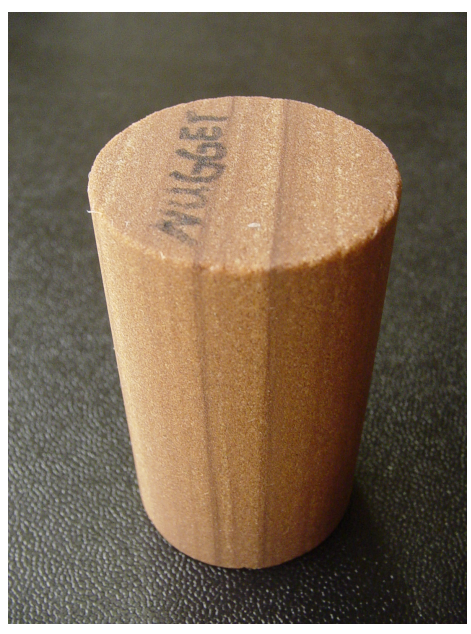

(a)

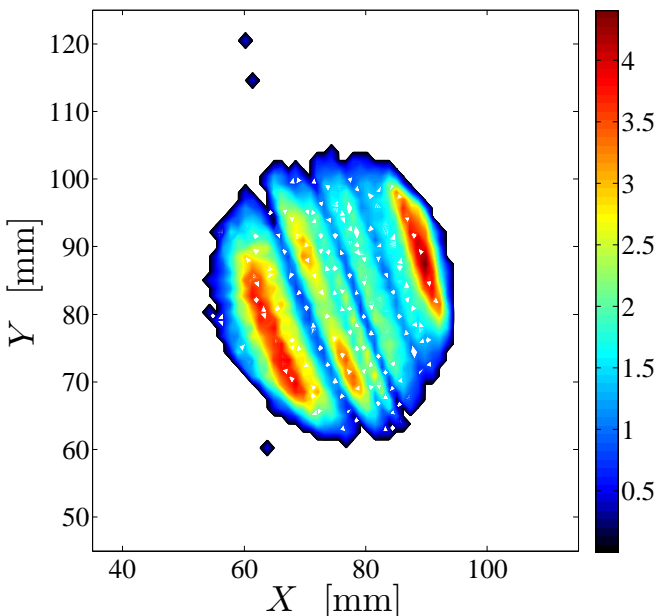

(b)

FIG. 3.5: A heterogeneous rock cores with red-brown quartz bedding plane structure (a). 2D spin echo image of this rock plug (b). The result is $128 \times 128$ with a resolution of $1.2 \mathrm{~mm}$. The unit of the MR imagine is $\mu \mathrm{V} / \mathrm{kHz}$.

\subsection{Tortuosity $T$ and permeability $\kappa$}

Tortuosity $T$ describes the sinuosity and interconnectedness of the pore channel/network [176]. As can been seen in Fig. 3.6 (a), it is typically defined by the square of ratio between sample length $L$ and flow-path length $L_{\mathrm{T}}: T=\left(L / L_{\mathrm{T}}\right)^{2}$.

Because of the sensitivity to spin-bearing particle diffusion process, NMR measurement can yield tortuosity information of porous materials. When liquid/gas molecules diffuse in the pore space, their mobility will be restricted by the boundary of pore walls. The effective diffusion coefficient is a function of the observation time and it can be measured by PFG NMR techniques. The attenuation of the apparent diffusion coefficient would 
follow the two-point Padé approximation [15]:

$$
\begin{aligned}
\frac{D(\Delta)}{D_{0}} & =1-\left(1-\frac{1}{F \phi}\right) \\
& \times \frac{\frac{4 \sqrt{D_{0} \Delta}}{9 \sqrt{\pi}} \frac{S}{V}+\left(1-\frac{1}{F \phi}\right) \frac{D_{0} \Delta}{D_{0} \theta}}{\left(1-\frac{1}{F \phi}\right)+\frac{4 \sqrt{D_{0} \Delta}}{9 \sqrt{\pi}} \frac{S}{V}+\left(1-\frac{1}{F \phi}\right) \frac{D_{0} \Delta}{D_{0} \theta}},
\end{aligned}
$$

where $\Delta$ is the diffusion observation time, $F$ is the electrical formation factor and defined by $\sigma_{\text {brine }} / \sigma_{\text {brine-saturated rock }} \phi$ is the sample porosity. The product of $F$ and $\phi$ refers to the tortuosity of rock $T . \sqrt{D_{0} \theta}$ is the heterogeneity length scale of the medium, which is expected to be proportional to the grain size. It will be typically much larger than the diffusion length $\sqrt{6 D_{0} \Delta}$ in rocks. As can be seen in Fig. $3.6(\mathrm{~b})$, the tortuosity $T$ can be readily extracted by fitting these time-dependent diffusion coefficient data points.

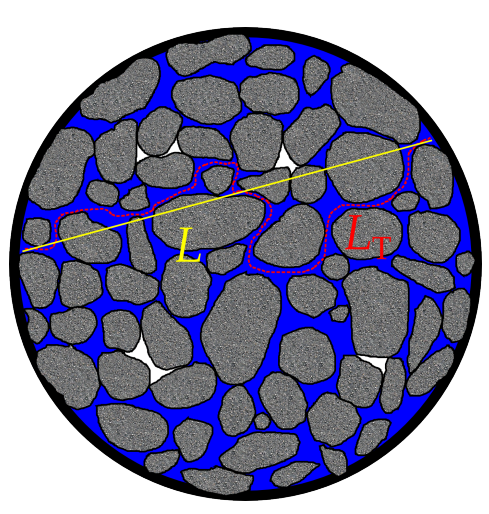

(a)

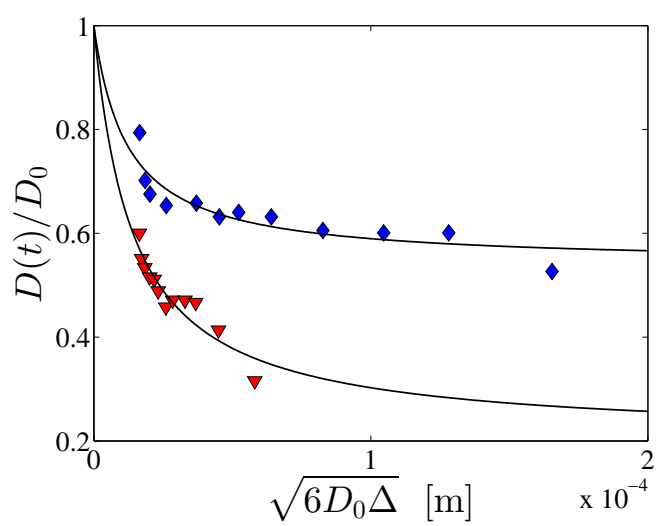

(b)

FIG. 3.6: Tortuosity definition (a) and normalized time-dependent diffusion coefficient of sandstone (red triangle) and limestone (blue diamond) core plugs plotted versus diffusion length $\sqrt{6 D_{0} \Delta}(\mathrm{b}) . \Delta$ is diffusion observation time which is varied during each steps of this experiment. Solid curves are Padé approximant fitting Eq. 3.6. The obtained $T$ is 4.88 in sandstone and 1.84 in limestone.

Permeability $\kappa$ characterizes the ease of fluid flowing through porous 
media and can be conceptually thought as the cross-sectional area of effective pore channels [161]. Therefore, permeability has an unit of length squared ${ }^{1}$.

Within the extensive NMR research work in porous media, it is believed that permeability strongly links to porosity $\phi$ and relaxation time (which relates to pore size) of porous materials $[5,16-18]$. Typically, there are two empirical models utilized to predict permeability from NMR experimental results. The first one is suggested in [19]:

$$
\kappa=c \cdot \phi^{4} \cdot\left[\frac{\mathrm{FFI}}{\mathrm{BVI}}\right]^{2}
$$

where $c$ is an empirical factor related to the lithology of rocks, FFI is Free Fluid Index and BVI is Bulk Volume Irreducible of fluid in porous materials. As shown in Fig. 3.7 (a), the fractions of these two volumes can be obtained by comparing NMR relaxation distributions before and after the centrifuge experiments.

Another pore system model is suggested in [20-22] and defined by:

$$
\kappa=c \cdot \phi^{4} \cdot T_{2 l m}^{2}
$$

As indicated by a dashed line in Fig. 3.7 (b), $T_{2 l m}$ is the logarithmic mean value of the acquired relaxation distributions and is defined as:

$$
\log _{10}\left(T_{2 l m}\right)=\frac{\sum\left[M_{0 i} \cdot \log _{10}\left(T_{2 i}\right)\right]}{\sum M_{0 i}}
$$

where $M_{0 i}$ represents the probability (amplitude) of the $i$-th $T_{2}$ component. Both models have been utilized in laboratory core analysis and can be further adopted in down-hole NMR well logging to estimate in-situ formation permeabilities.

There are plenty of other independent NMR method to characterize per-

\footnotetext{
${ }^{1}$ Common unit in petroleum engineering is Darcy or miliDarcy, $1 \mathrm{mD} \approx 10^{-3} \mu \mathrm{m}^{2}$.
} 
meability of rock cores, for instance by means of time-dependent diffusion [120, 177, 178], propagator and velocity measurements [179].

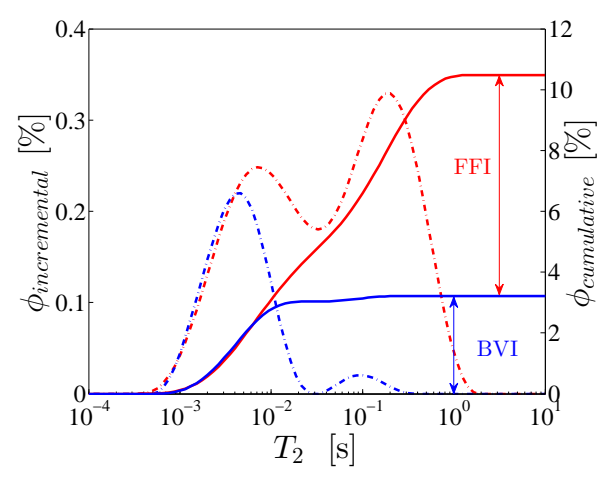

(a)

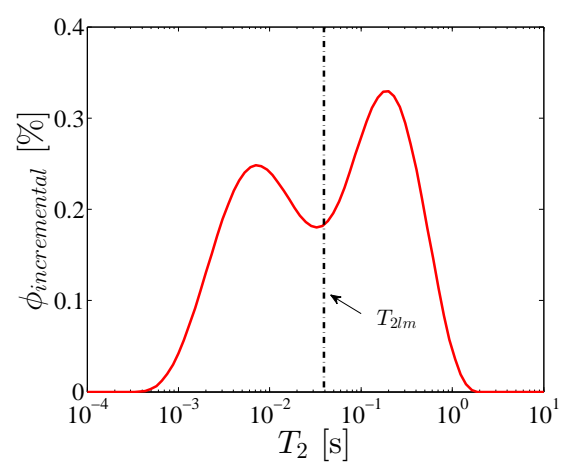

(b)

FIG. 3.7: (a) Red curves are the relaxation distributions before centrifuge and the blue curves are the results after centrifuge measurement, respectively. The amount of FFI and BVI can be determined from the cumulative plateaus before and after the centrifuge and used for the permeability estimation from Eq. 3.7. (b) Permeability estimation from Eq. 3.8. Vertical dashed line corresponds to the log-mean value of relaxation distribution.

\subsection{Fluids identification and wettability}

Fluid typing: Underground resources exploitation in petroleum, geothermal and hydrological fields always involves multiple types of fluids/gas. Therefore, it is important for petrophysicists to understand the fluid types and their viscous properties. A common NMR technique to identify fluids is $1 \mathrm{D}$ or 2D diffusometry since it is inherently sensitive to the molecular mobility $[9,11,24-26,39,40,49]$. Therefore, it allows one to identify different fluids and quantify individual fluid saturation in rock samples. Moreover, $T_{1} / T_{2}$ ratio can also be used as an indicator to identify the fluid types [180].

Wettability is the tendency of a fluid to spread on and preferentially adhere to or "wet" a solid surface in the presence of other immiscible 


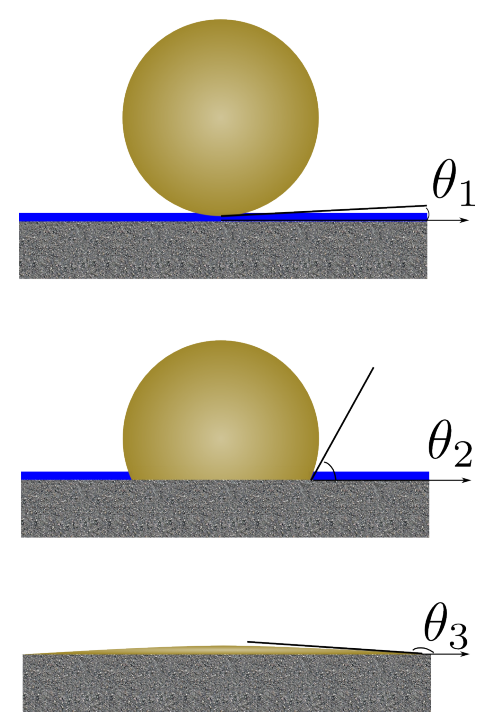

(a)

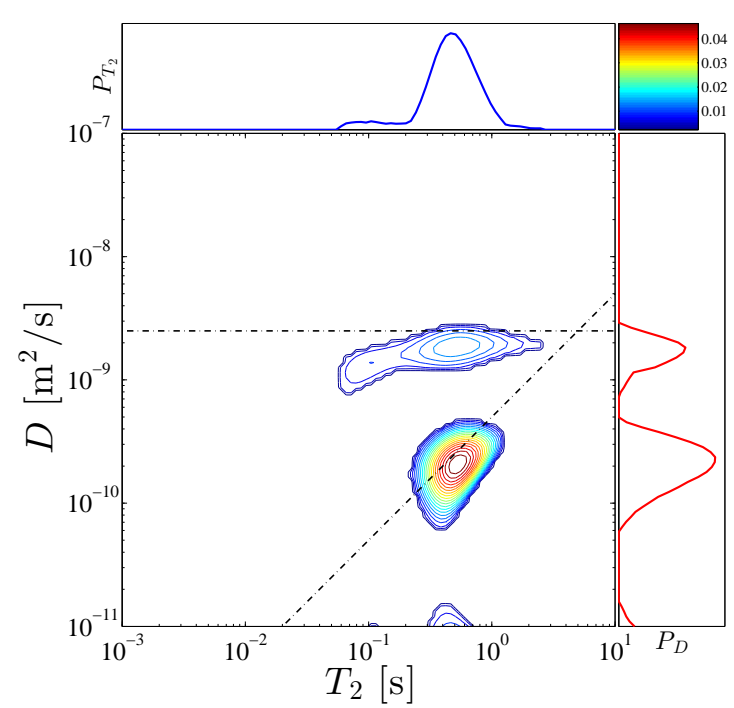

(b)

FIG. 3.8: (a) Wettability defined by a contact angle $\theta$. The yellow sphere and the blue layer represent oil droplet and water film. $\theta_{1}, \theta_{2}$ and $\theta_{3}$ indicate the waterwetting, mixing-wetting and oil-wetting, respectively. (b) $2 \mathrm{D} D-T_{2}$ correlation distribution of an oil-bearing rock core after water-flooding experiment. The horizontal dashed line represents a self-diffusion coefficient value of $2.3 \times 10^{-9}$ $\mathrm{m}^{2} / \mathrm{s}$. The slope dash line represents hydrocarbon correlation line of $D=5 \times 10^{-10}$ $T_{2}$. The water distribution exhibits a tendency of smaller $D$ values with decreasing $T_{2}$, which indicates the existence of water sinks close to solid surface and therefore implies water-wet status of this rock plug.

fluids [181]. It is critical to have the knowledge of reservoir wettability at hand because it influences residual oil saturation, relative permeability, and capillary pressure. A popular laboratory indicator of wettability is contact angle $\theta$ measured in water-oil-solid systems as shown in Fig. 3.8 (a).

Traditionally, wettability of partially saturated rocks can be determined by comparing either $T_{1}$ or minimal diffusion-effect $T_{2}$ distributions of fluid measured in rocks with the result from bulk fluid (i.e., outside the rocks) [182-190]. If the fluid has a comparable behavior inside and outside of the rock core, it would be non-wetting fluid, otherwise, it will be a wetting-fluid if it sticks to the surface of pore matrix and experiences surface relaxation 
effect. As a more comprehensive technique, 2D $D-T_{2}$ NMR correlation result shown in Fig. 3.8 (b) indicates the fluid present in different regions of the pore space and their interactions with the solid surface wall, thus yielding the wetting status of rock samples [24, 25, 191].

\subsection{Flooding process}

Rock core flooding experiments are important to understand underground fluid flow, saturation and recovery mechanisms. In order to perform flooding experiments in the laboratory, a specialized core holder with strong and sustainable materials needs to be implemented first to maintain the core plug in pressurized conditions.

In particular, some more special design requirements occur for NMRfriendly rock holder in flooding experiments. Its body and other accessories should be at least non-magnetic materials, such as polymer or ceramic, to avoid distorting NMR signal [134,192, 193]. Even though the holders could be metallic vessels considering its relatively low cost, the induced eddy currents need to be measured and removed from the acquired signal using mathematical operation during the data analysis [194-199].

In order to provide the required temperatures for the loaded rock core, a typical way is wrapping heating tapes around the rock core in the pressurized holder. However, this approach does not fit the NMR case since it will perturb RF pulses and pulsed field gradients. Therefore, a special fluid which does not contribute to the NMR signal should be used instead as a confining fluid which circulates around the loaded sample. The pressure and temperature can then be controlled via pressurizing and heating up the confining fluid. A more detailed introduction about such instrument set-up will be discussed in Chapter 6 .

MRI techniques are routinely applied to visualize and quantify flow processes in rock cores. To obtain "real-time" information, fast MRI techniques which have been developed for medical diagnosis have been modified and 
adopted for petrophyscial applications [126, 127, 200]. Meanwhile, some other means, such as flow propagator and relaxation time distributions are employed to observe the fluid transport and saturation in rocks during flooding process [74, 201-208]. While performing NMR measurements, different types of fluids and/or gases, for instance brine, $\mathrm{CO}_{2}$ and deuterium oxide $\mathrm{D}_{2} \mathrm{O}$, can be pressurized and flooded through core plugs, in order to study the recovery mechanism of reservoir rock cores [12, 209-220]. 


\section{Chapter 4}

\section{Pore length and surface relaxivity}

In this chapter, we lay out the theory needed for pore length scales and surface relaxivity determination in rock cores. Pore size is measured relying on high excited relaxation eigenmode in pore space. One-dimensional (1D) results from different types of rock cores at two field strengths proved the feasibility of this approach. Furthermore, the ground and higher relaxation eigenmodes can be coupled and measured in 2D experiments, which provides the distribution of pore length correlated to NMR relaxation times. Subsequently, surface relaxivities of rock cores can be extracted from the obtained $2 D$ eigenmode correlation maps $[82,83]$.

\subsection{Pore length determination}

\subsubsection{Review of pore length determination using the first excited mode $(n=1)$}

When molecular diffusion happens within both volume relaxivity $\nu$ and surface spin absorbing wall characterized by $\rho$, the local magnetization density $m(\mathbf{r}, t)$ in certain pore volume satisfies the following equation:

$$
\frac{\partial}{\partial t} m(\mathbf{r}, t)=D \nabla^{2} m(\mathbf{r}, t)-\nu m(\mathbf{r}, t)
$$


with a pore/matrix boundary condition of $\left.(\hat{n} \cdot D \nabla m+\rho m)\right|_{S}=0$. Here $\mathbf{r}$ is the spin position, $D$ is the diffusion coefficient of the saturating fluid and is considered spatially constant, and $\hat{n}$ is the normal unitary vector outward pointing from the pore/matrix interface. This formalism is also the fundamental of many NMR simulation tools in porous materials, such as random walk [221, 222] and Multiple Correlation Function (MCF ) [164].

Eq. 4.1 is a typical Partial Differential Equation (PDE) if neglecting the effect from bulk relaxation and the general solution can be obtained by separating the variables $\mathbf{r}$ and $t$. The result is given as:

$$
m(\mathbf{r}, t)=\sum_{n=0}^{\infty} A_{n} \varphi_{n}(\mathbf{r}) \mathrm{e}^{-\frac{t}{\tau_{1,2}^{n}}}
$$

where $\varphi_{n}$ are orthogonal, normalized eigenfunctions of the diffusion equation and $1 / \tau_{1,2}^{n}$ are the corresponding eigenvalues when the magnetization is along the longitudinal axis or in the transverse plane [163]. While the ground eigenfunction $\varphi_{0}$ is approximately constant, the higher eigenfunctions will oscillate in pore space. The eigenmode amplitude $A_{n}$ can be calculated using the orthogonal property of eigenfunctions and the initial magnetization:

$$
A_{n}=\frac{1}{V} \int m(\mathbf{r}, 0) \varphi_{n}(\vec{r}) d V
$$

Considering the behavior of eigenfunctions in the pore space, the ground mode amplitude will be approximately $m_{0} / V$ but the amplitude of higher modes will be small assuming a uniform magnetization profile.

For a spherical pore space (a common assumption in porous media research) the decay times $\tau_{n}$ for spherical pore space are given as:

$$
\tau_{1,2}^{n} \approx\left\{\begin{array}{lll}
\frac{a}{6 \rho_{1,2}} & \text { when } \quad n=0 \\
\frac{a^{2}}{4 D \zeta_{n}^{2}} & \text { when } n \geqslant 1
\end{array}\right.
$$

under the condition of fast diffusion region $(\rho a / D \ll 1)$, where the $\zeta_{n}$ are the positive roots for the equation $1-\zeta_{n} \cot \zeta_{n}=\rho a / 2 D[163,164,223]$. 
Here, $a$ is the diameter of the sphere and $\rho$ is the surface relaxivity affecting longitudinal or transverse magnetization whichever one is involved during the diffusion observation time. The decay time of the ground mode $\tau_{1,2}^{0}$ will always be larger under this condition, than $\tau_{1,2}^{n}$ with $n \geqslant 1$.

Since the detected signal is the integral of the magnetization over the pore space, the result will be a multi-exponential decay weighted by the relative intensity of each mode [163]:

$$
m(t)=\int m(\mathbf{r}, t) d V=m_{0} \cdot \sum_{n=0}^{\infty} I_{n} \mathrm{e}^{-\frac{t}{\tau_{1,2}^{n}}}
$$

where $I_{n}=\left[\int \varphi_{n} d V\right]^{2}$, is the relative intensity of $n$-th mode of the diffusion equation. As an example, the eigenvalues and relative intensities of different eigenmodes in analytical solution of sphere is given in Fig. 4.1.

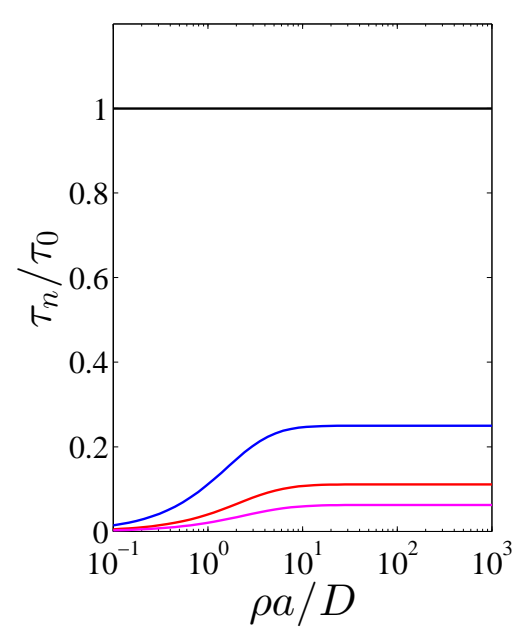

(a)

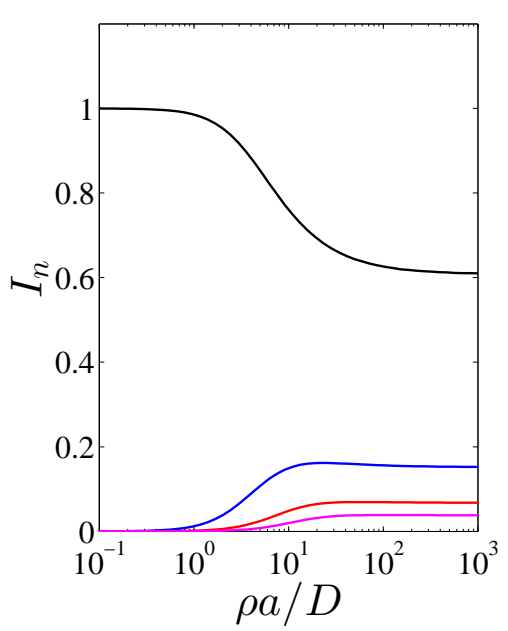

(b)

FIG. 4.1: The eigenvalues (a) and relative intensities (b) of eigenmodes in an analytical solution of sphere suggested by Ref. [163]. The characteristics of the modes from $n=0$ to $n=3$ are denoted by the curves with black, blue, red and pink colors, respectively. Within the condition of fast diffusion limits, the eigensolution will consist of smaller eigenvalues and lower relative intensities with increasing order of eigenmodes. 
As seen from Eq. 4.4, the decay time of the ground mode $(n=0)$ depends on the pore length $a$ and surface relaxivity $\rho$, while the high modes $(n \geqslant 1)$ of the diffusion equation are independent of $\rho$ and therefore are more suitable for determining the pore length even in the presence of surface relaxivity heterogeneity. However, the relative intensities of the high modes are much weaker compared to the intensity of ground mode as shown in Fig. 4.1 (b). In order to take advantage of the high modes for the detection of pore length scale, the contribution of the high modes to the signal must be enhanced.

One efficient approach to enhance the contributions from the first excited mode $(n=1)$ in the presence of spatially distributed internal magnetic field $B_{i n}$ was developed for porous media [157, 224]. The $1 D$ signal pulse sequence and reference pulse sequence used in this technique are shown in Fig. 4.2.
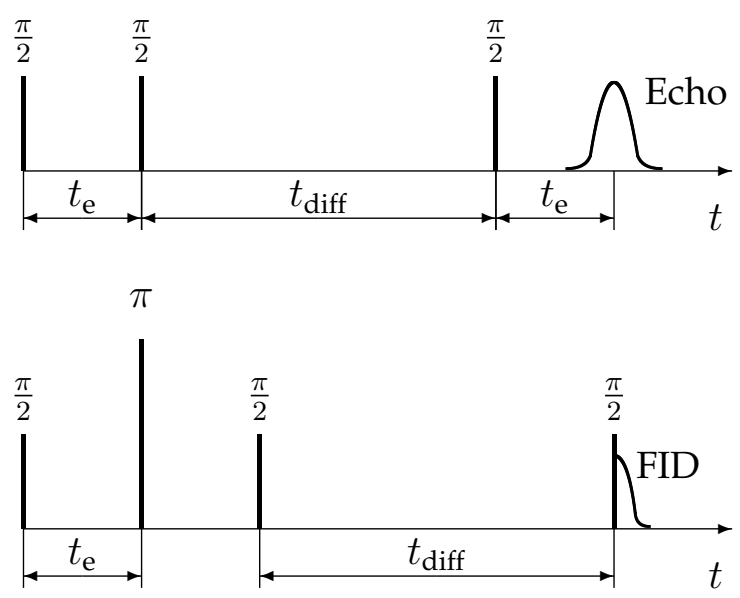

FIG. 4.2: 1D signal pulse sequence (top) and reference pulse sequence (bottom) are used to detect high eigenmode in porous media [157]. Here $t_{\mathrm{e}}$ is the encoding period and $t_{\text {diff }}$ is the observation time of molecular diffusion. The phase cycling used in the signal and reference pulse sequences is given in Tab. 4.1. The intensities of the acquired stimulated echoes and FIDs can then be observed as a function of $t_{\text {diff. }}$

In the 1D signal pulse sequence, the first $\pi / 2$ RF pulse rotates the longitudinal magnetization in the transverse plane. During the encoding period 
of $t_{\mathrm{e}}$, the magnetization in pore space will be modulated with an encoding phase $\Phi$ in the presence of the induced magnetic fields $B_{i n}$ which varies in space:

$$
m\left(\mathbf{r}, t_{\mathrm{e}}\right)=m(\mathbf{r}, 0) \mathrm{e}^{-i \Phi}=m(\mathbf{r}, 0) \mathrm{e}^{-i \gamma B_{i n}(\mathbf{r}) t_{\mathrm{e}}} .
$$

Here, the nuclei should be considered as immobile during the encoding time $t_{\mathrm{e}}$ for the modulation of the magnetization profile, otherwise an integral of the internal magnetic fields has to be used instead of $B_{i n}(r)$ in the exponential term.

The second $\pi / 2$ RF pulse stores the dephased magnetization back to the longitudinal direction. After this, the magnetization evolution during the observation time $t_{\text {diff }}$ will undergo spin-lattice relaxation $T_{1}$. By choosing the phase of the second $\pi / 2$ RF pulse to be incremented by $90^{\circ}$ as compared to the first $\pi / 2$ RF pulse, one can select only the sine component of the magnetization and store it back in the longitudinal direction [225]. This component is of interest because it allows us to suppress the contribution from the ground $T_{1}$ mode, since the initial magnetization is $m\left(\vec{r}, t_{\mathrm{e}}\right)$ and the amplitude of ground mode $A_{0}=(1 / V) \cdot \int m\left(\vec{r}, t_{\mathrm{e}}\right) \varphi_{0} d V=(1 / V)$. $\int m(\vec{r}, 0) \sin \Phi \varphi_{0} d V \approx 0$ according to Eq. 4.3. The phase cycling of this $1 \mathrm{D}$ signal pulse sequence is given in the left of Table. 4.1. The phase of the first three $\pi / 2$ pulses, $\phi_{1}, \phi_{2}, \phi_{3}$, and the acquisition phase, $\phi_{A c q}$, are cycled through the experiment to excite the higher $T_{1}$ mode of relaxation time in the $1^{\text {st }}$ domain.

In order to characterize the pore size distribution in an explicit way, the first $T_{1}$ mode $(n=1)$ should be excited predominantly. This is achieved by satisfying the weak dephasing condition $\gamma \Delta B_{i n} t_{\mathrm{e}}<1$, where $\Delta B_{\text {in }}$ represents the square root of the second moment of internal magnetic fields $B_{\text {in }}$ [225]. Hence the signal equation for the acquired stimulated echo amplitude $E\left(t_{\text {diff }}\right)$ will be:

$$
E\left(t_{\text {diff }}\right) \approx m_{0} \cdot\left(I_{0} \mathrm{e}^{-t_{\text {diff }} / \tau_{1}^{0}}+I_{1} \mathrm{e}^{-t_{\text {diff }} / \tau_{1}^{1}}\right)
$$


where, $I_{0}$ and $I_{1}$ are the relative intensities of the ground and first $T_{1}$ mode, respectively. Similarly, $\tau_{1}^{0}$ and $\tau_{1}^{1}$ are the respective decay times. According to Eq. $4.2,4.3$ and 4.5, the relative intensity of the ground $T_{1}$ mode $I_{0}$ would be:

$$
I_{0}=\int \varphi_{1}^{0} d V \cdot \int \sin \Phi \varphi_{1}^{0} d V
$$

To isolate the contribution of the ground $T_{1}$ mode during the observation time $t_{\text {diff, }}$ the $1 \mathrm{D}$ reference pulse sequence is applied. The $\pi$ RF pulse in the middle of the magnetization preparation period cancels the phase accumulation due to $B_{i n}$. The phase cycling of this 1D signal pulse sequence is given in the right of Table. 4.1. The phase of the first three $\pi / 2$ pulses, $\phi_{1}, \phi_{2}, \phi_{3}$, the phase of $\pi$ pulse $\phi_{4}$, and the acquisition phase, $\phi_{A c q}$, are cycled through the experiment to excite the ground $T_{1}$ mode of relaxation time in the $1^{\text {st }}$ domain. As a result, the magnetization after the second $\pi / 2 \mathrm{RF}$ pulse is uniform in the pore space, giving the initial condition $m\left(\vec{r}, 2 t_{\mathrm{e}}\right)=m\left(2 t_{\mathrm{e}}\right) / V$. In this case, the amplitude of the ground mode $A_{0}=(1 / V) \cdot \int m\left(\vec{r}, 2 t_{\mathrm{e}}\right) \varphi_{0} d V \approx m\left(2 t_{\mathrm{e}}\right) / V$ according to Eq. 4.3. The ground mode dominates in the diffusion equation again and the FID attenuation is only caused by the ground $T_{1}$ mode during $t_{\text {diff: }}$ :

$$
R\left(t_{\text {diff }}\right) \approx m_{0} \cdot \mathrm{e}^{-t_{\text {diff }} / \tau_{1}^{0}}
$$

To eliminate the contribution from ground $T_{1}$ mode in Eq. 4.7, the value of $I_{0}$ is determined using the ratio $E\left(t_{\text {diff }}\right) / R\left(t_{\text {diff }}\right)=I_{0}$ at long $t_{\text {diff. Using }}$ $I_{0}$ for scaling of $R\left(t_{\text {diff }}\right)$ such that its amplitude matches the contribution of the ground eigenmode in Eq. 4.7, one is able to subtract Eq. 4.9 from Eq. 4.7 as $E\left(t_{\text {diff }}\right)-I_{0} \cdot R\left(t_{\text {diff }}\right)$ which results in:

$$
S\left(t_{\text {diff }}\right) \approx m_{0} \cdot I_{1} \exp \left(-\frac{t_{\text {diff }}}{\tau_{1}^{1}}\right)=m_{0} \cdot I_{1} \exp \left(-\frac{4 t_{\text {diff }} D \zeta_{1}^{2}}{a^{2}}\right) .
$$

$S\left(t_{\text {diff }}\right)$ expresses the signal decay mainly due to the first $T_{1}$ mode (when neglecting higher orders), where the exponential decay term $4 D \zeta_{1}^{2} / a^{2}$ gives 
us direct access to the the pore length as per Eq. 4.4.

Since most rocks have a distribution of pore lengths, the measured signal will be the sum of the contributions from all pores within the sample. As each pore with length $a$ obeys Eq. 4.10, the signal attenuation of the sample will follow a multi-exponential decay

$$
S\left(t_{\text {diff }}\right)=I_{1} \int F(a) \exp \left(-\frac{4 t_{\text {diff }} D \zeta_{1}^{2}}{a^{2}}\right) d a
$$

where $F(a)$ is the pore length distribution of rock samples. Typically a 1D numerical inversion method is used to obtain the $\tau_{1}^{1}$ distribution from the data set, and the pore length scales of rock cores can thus be acquired via $a \approx 2 \zeta_{1} \sqrt{D \tau_{1}^{1}}$ from Eq. 4.4 ( $\zeta_{1} \approx 4.49$ in the condition of the fast diffusion limits [223]). It is worth noting that the even higher modes $(n>1)$ actually still contribute to the signal decay in Eq. 4.10, although they are negligible compare to the first excited mode. Some other pore parameters, such as pore shape or curvature, probably can be studied from the contribution of these modes $(n>1)$ in the data set using sophisticated math algorithm, which is beyond the discussion of this work.

With the above knowledge at hand, we can now discuss the high eigenmode detection at low-field and the implementation of 2D eigenmodes correlation techniques.

\subsubsection{Strategies of the first excited mode detection in low- field NMR}

In low-field NMR context for instance a industrial benchmark field strength of $0.05 \mathrm{~T}$ (corresponding to $2 \mathrm{MHz}{ }^{1} \mathrm{H}$ Larmor frequency), the internal magnetic fields $B_{i n}$ are weak compared to high-field, since the range of $B_{\text {in }}$ is proportional to the static magnetic field $B_{0}$ according to Eq. 3.2 [144]. Even though $B_{\text {in }}$ are weak, they are still accessible [145] and can be utilized to build a non-uniform magnetization profile in the pore 
Table 4.1: Phase cycling for the 1D signal pulse sequence (Left) and reference pulse sequence (Right). $\phi_{1}, \phi_{2}, \phi_{3}$ and $\phi_{4}$ and $\phi_{A c q}$ are the phase of the first three $\pi / 2$ pulses, $\pi$ pulse and the acquisition phase, respectively.

\begin{tabular}{cccc}
\hline$\phi_{1}$ & $\phi_{2}$ & $\phi_{3}$ & $\phi_{A c q}$ \\
\hline$\pi / 2$ & 0 & 0 & $\pi / 2$ \\
$3 \pi / 2$ & 0 & 0 & $3 \pi / 2$ \\
$\pi / 2$ & $\pi$ & 0 & $3 \pi / 2$ \\
$3 \pi / 2$ & $\pi$ & 0 & $\pi / 2$ \\
\hline
\end{tabular}

\begin{tabular}{ccccc}
\hline$\phi_{1}$ & $\phi_{2}$ & $\phi_{3}$ & $\phi_{4}$ & $\phi_{A c q}$ \\
\hline 0 & 0 & 0 & $\pi / 2$ & $\pi / 2$ \\
$\pi$ & 0 & 0 & $\pi / 2$ & $3 \pi / 2$ \\
0 & $\pi$ & 0 & $\pi / 2$ & $3 \pi / 2$ \\
$\pi$ & $\pi$ & 0 & $\pi / 2$ & $\pi / 2$ \\
0 & 0 & 0 & $3 \pi / 2$ & $\pi / 2$ \\
$\pi$ & 0 & 0 & $3 \pi / 2$ & $3 \pi / 2$ \\
0 & $\pi$ & 0 & $3 \pi / 2$ & $3 \pi / 2$ \\
$\pi$ & $\pi$ & 0 & $3 \pi / 2$ & $\pi / 2$ \\
\hline
\end{tabular}

space. In turn this gives us the ability to enhance relative intensity of the high eigenmode.

One possible strategy for the detection of high eigenmodes at lowfield is by elongating $t_{\mathrm{e}}$. This allows the encoding phase $\Phi$ to evolve to the point of detection, since the strength of the encoding phase $\Phi$ is characterized by $\gamma \Delta \chi B_{0} t_{\mathrm{e}}$. If $t_{\mathrm{e}}$ is short, the encoding phase will not evolve significantly and the magnetization at different positions of the pore will appear uniform. This will lead to the dominance of the ground mode in the solution of the diffusion equation in the pore space as seen in Eq. 4.2. By extending the encoding period $t_{\mathrm{e}}$, the spins will accumulate sufficient phase difference due to the precession in the internal magnetic fields $B_{i n}$, giving rise to the non-uniform magnetization profile. This will enhance the contribution of high eigenmodes in Eq. 4.2, thus allowing a pore length scale determination at low-field NMR. However, $t_{\mathrm{e}}$ can not be set too long, because the weak dephasing condition must be satisfied to maintain the dominant contribution from the first $T_{1}$ mode. Secondly, a long $t_{\mathrm{e}}$ will limit pore length detection to $\sqrt{D t_{\mathrm{e}}}$ due to molecular diffusion. 
Table 4.2: Eigenmode measured in different 1D NMR experiments

\begin{tabular}{lcc}
\hline Pulse sequence & Ground mode & First mode \\
\hline CPMG [33, 34] & $\varphi_{2}^{0}, \tau_{2}^{0}$ & $\times$ \\
Small $\alpha$ pulse train [32] & $\varphi_{1}^{0}, \tau_{1}^{0}$ & $\times$ \\
Inversion Recovery & $\varphi_{1}^{0}, \tau_{1}^{0}$ & $\times$ \\
DDIF-signal [157] & $\varphi_{1}^{0}, \tau_{1}^{0}$ & $\varphi_{1}^{1}, \tau_{1}^{1}$ \\
DDIF-reference [157] & $\varphi_{1}^{0}, \tau_{1}^{0}$ & $\times$ \\
\hline
\end{tabular}

\subsubsection{Summary of eigenmode involved in 1D NMR}

As indicated in Fig. 4.1 (b), the contribution of the ground eigenmode dominates in the NMR signals within a uniform magnetization profile over the pore space. Therefore, for most of the routine NMR measurements such as CPMG, inversion/saturation recovery, and small tip angle pulse train, the involved eigenmode of the spin relaxation during the experiments are always the ground $T_{2}$ and $T_{1}$ mode. The higher modes with oscillating eigenfunctions, however, are hardly accessible. By utilizing the induced magnetic fields distributed in the pore space, the distribution of the initial magnetization profile can be modulated. Thus, the relative intensities of the eigenmodes are adjusted and the high mode containing abundant pore morphology information is feasible to be detected in 1D NMR experiments.

\subsection{D relaxation eigenmode correlation}

Recent developed 2D NMR techniques provide more detailed insight of heterogeneous material properties through correlation and exchange patterns as compared to their 1D counterparts, such as characterizing the internal structure and molecular diffusion behavior in restricted geometries [9, 63-68]. For a typical 2D correlation experiment, two separated periods $P_{\alpha}$ and $P_{\beta}$ are varied in the pulse sequence involving two different spin dynamics. Then the spatial magnetization evolution can be characterized by two basis sets corresponding to the individual eigenfunctions $\varphi_{\alpha}^{m}, \varphi_{\beta}^{n}$, 
and decay times $\tau_{\alpha}^{m}, \tau_{\beta}^{n}$. Therefore, the signal acquired in the 2D experiment is the integral of the spatial magnetization in the pore space [226, 227]:

$$
\begin{aligned}
m\left(P_{\alpha}, P_{\beta}\right) & =\int m\left(\mathbf{r}, P_{\alpha}, P_{\beta}\right) d V \\
& =m_{0} \cdot \sum_{m, n} I_{m, n} \exp \left(-\frac{P_{\alpha}}{\tau_{\alpha}^{m}}\right) \exp \left(-\frac{P_{\beta}}{\tau_{\beta}^{n}}\right)
\end{aligned}
$$

where $I_{m, n}=\int \varphi_{\beta}^{n}(\mathbf{r}) d V \cdot \int \varphi_{\beta}^{n}(\mathbf{r}) \varphi_{\alpha}^{m}(\mathbf{r}) d V \cdot \int \varphi_{\alpha}^{m}(\mathbf{r}) d V$, which is the correlation intensity of $m$-th eigenmode in the first period $P_{\alpha}$ and $n$-th eigenmode in the second period $P_{\beta}$.

\subsubsection{Correlation of ground $T_{2}$ and first $T_{1}$ mode}

As can be seen in Eq. 4.4, if the detection of the first excited $T_{1}$ mode and ground $T_{2}$ mode are combined in a $2 \mathrm{D}$ experiment, it will provide the information of pore length, $a$ and pore surface relaxivity, $\rho_{2}$ simultaneously. To achieve this, a CPMG pulse train can be performed after the aforementioned 1D pulse sequences in Fig. 4.2 to correlate the pore length scale with the transverse relaxation time $T_{2}$. The pulse sequences are shown in Fig. 4.3.

During this experiment, the spins will experience both $T_{1}$ and $T_{2}$ dynamics. According to Eq. 4.12, if neglecting the $T_{2}$ effect during the $1^{\text {st }}$ domain, the acquired data using the $2 \mathrm{D}$ signal pulse sequence can be expressed as:

$$
\begin{aligned}
E\left(t_{\text {diff }}, N T_{\mathrm{E}}\right) & =\int m\left(\mathbf{r}, t_{\text {diff }}, N T_{\mathrm{E}}\right) d V \\
& =m_{0} \cdot \sum_{m, n} I_{m, n} \exp \left(-\frac{t_{\text {diff }}}{\tau_{1}^{m}}\right) \exp \left(-\frac{N T_{\mathrm{E}}}{\tau_{2}^{n}}\right)
\end{aligned}
$$

where $\tau_{1}^{m}$ and $\tau_{2}^{n}$ are the decay times of $m$-th $T_{1}$ mode and $n$-th $T_{2}$ mode, and $I_{m, n}$ is the correlation intensity of these two modes.

The $1^{\text {st }}$ domain of the 2D correlation sequence pulse sequence is adopted 


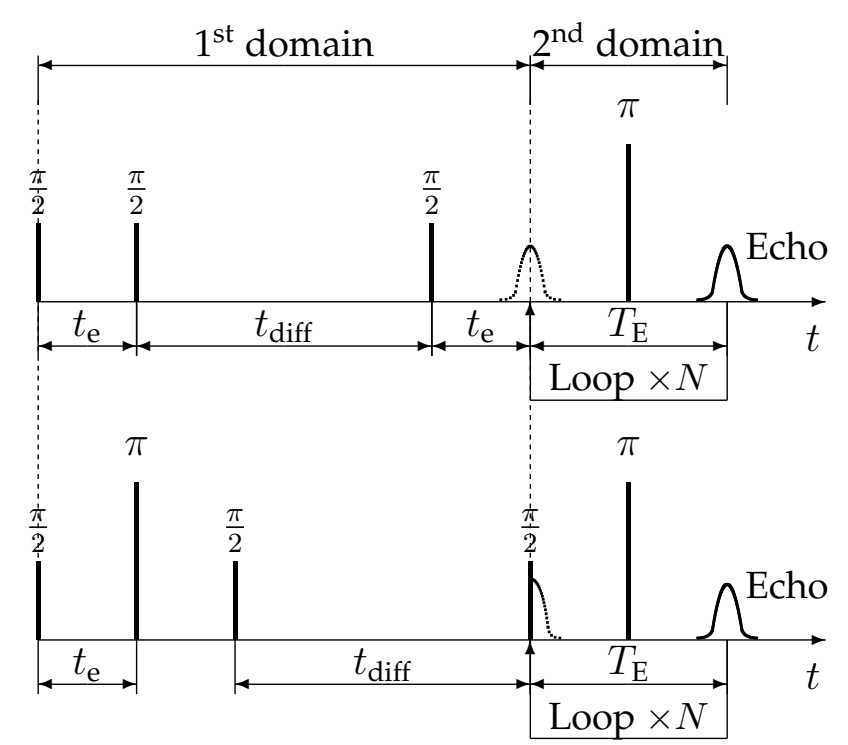

FIG. 4.3: 2D signal (top) and reference (bottom) pulse sequence used to correlate the high and ground eigenmode of diffusion equation. $T_{\mathrm{E}}$ is echo spacing and $N$ is echo number of CPMG pulse train. The phase cycling used in this $2 \mathrm{D}$ pulse sequence is given in Tab. 4.3. The amplitudes of the echo trains from these two pulse sequences can then be acquired under a series of $t_{\text {diff. }}$.

from 1D case (see top in Fig. 4.3) and encodes the information from the ground and first $T_{1}$ mode. At the end of the $1^{\text {st }}$ domain, a stimulated echo is observed as the magnetization is refocused, leading to a situation where the ground $T_{2}$ mode dominates the second stage of the $2 \mathrm{D}$ pulse sequence. The phase cycling of this $2 \mathrm{D}$ pulse sequence is given in the left of Table. 4.3. The phase of the first three $\pi / 2$ pulses, $\phi_{1}, \phi_{2}, \phi_{3}$, the phase of $\pi$ pulse $\phi_{4}$, and the acquisition phase, $\phi_{A c q}$, are cycled through the experiment to excite the higher $T_{1}$ mode of relaxation time in the $1^{\text {st }}$ domain and ground $T_{2}$ mode in the second period. As a result, the acquired data from 2D signal pulse sequence can be approximately expressed as:

$$
\begin{aligned}
E\left(t_{\text {diff }}, N T_{\mathrm{E}}\right) & \approx m_{0} \cdot\left[I_{0,0} \exp \left(-\frac{t_{\text {diff }}}{\tau_{1}^{0}}\right) \exp \left(-\frac{N T_{\mathrm{E}}}{\tau_{2}^{0}}\right)\right] \\
& +m_{0} \cdot\left[I_{1,0} \exp \left(-\frac{t_{\text {diff }}}{\tau_{1}^{1}}\right) \exp \left(-\frac{N T_{\mathrm{E}}}{\tau_{2}^{0}}\right)\right]
\end{aligned}
$$


Table 4.3: Phase cycling for $2 \mathrm{D}$ ground $T_{2}$ and first $T_{1}$ mode correlation signal pulse sequence (Left) and reference pulse sequence (Right). $\phi_{1}, \phi_{2}, \phi_{3}$ and $\phi_{4}$ and $\phi_{\text {Acq }}$ are the phase of the first three $\pi / 2$ pulses, $\pi$ pulse and the acquisition phase, respectively.

\begin{tabular}{ccccc}
\hline$\phi_{1}$ & $\phi_{2}$ & $\phi_{3}$ & $\phi_{4}$ & $\phi_{\text {Acq }}$ \\
\hline$\pi$ & $\pi / 2$ & $\pi / 2$ & $\pi / 2$ & $\pi$ \\
0 & $\pi / 2$ & $\pi / 2$ & $\pi / 2$ & 0 \\
$\pi$ & $3 \pi / 2$ & $\pi / 2$ & $\pi / 2$ & 0 \\
0 & $3 \pi / 2$ & $\pi / 2$ & $\pi / 2$ & $\pi$ \\
$\pi$ & $\pi / 2$ & $\pi / 2$ & $3 \pi / 2$ & $\pi$ \\
0 & $\pi / 2$ & $\pi / 2$ & $3 \pi / 2$ & 0 \\
$\pi$ & $3 \pi / 2$ & $\pi / 2$ & $3 \pi / 2$ & 0 \\
0 & $3 \pi / 2$ & $\pi / 2$ & $3 \pi / 2$ & $\pi$ \\
\hline
\end{tabular}

\begin{tabular}{ccccc}
\hline$\phi_{1}$ & $\phi_{2}$ & $\phi_{3}$ & $\phi_{4}$ & $\phi_{A c q}$ \\
\hline 0 & 0 & 0 & $\pi / 2$ & $\pi / 2$ \\
$\pi$ & 0 & 0 & $\pi / 2$ & $3 \pi / 2$ \\
0 & $\pi$ & 0 & $\pi / 2$ & $3 \pi / 2$ \\
$\pi$ & $\pi$ & 0 & $\pi / 2$ & $\pi / 2$ \\
0 & 0 & 0 & $3 \pi / 2$ & $\pi / 2$ \\
$\pi$ & 0 & 0 & $3 \pi / 2$ & $3 \pi / 2$ \\
0 & $\pi$ & 0 & $3 \pi / 2$ & $3 \pi / 2$ \\
$\pi$ & $\pi$ & 0 & $3 \pi / 2$ & $\pi / 2$ \\
\hline
\end{tabular}

where the first term represents the contribution from the correlation of ground $T_{1}$ and $T_{2}$ modes, and the second term is the signal involving the first $T_{1}$ mode and ground $T_{2}$ mode correlation.

In order to calibrate the effect of ground $T_{1}$ mode in the first stage of this experiment, the aforementioned reference pulse sequence is followed by CPMG pulse train (see bottom in Fig. 4.3). The phase cycling of this $2 \mathrm{D}$ pulse sequence is given in the right part of Table. 4.3. The phase of the first three $\pi / 2$ pulses, $\phi_{1}, \phi_{2}, \phi_{3}$, the phase of $\pi$ pulse $\phi_{4}$, and the acquisition phase, $\phi_{\text {Acq }}$, are cycled through the experiment. As the effect of the internal magnetic fields is compensated by the $\pi$ RF pulse in the middle of the modulation period, only the ground $T_{1}$ mode is present during $t_{\text {diff }}$ and the acquired data from this $2 \mathrm{D}$ reference pulse sequence is described as:

$$
R\left(t_{\text {diff }}, N T_{\mathrm{E}}\right) \approx m_{0} \cdot \exp \left(-\frac{t_{\text {diff }}}{\tau_{1}^{0}}\right) \exp \left(-\frac{N T_{\mathrm{E}}}{\tau_{2}^{0}}\right)
$$

To extract the contribution from the high and ground-eigenmode correlation, the factor of $I_{0,0}$ needs to be calculated in order to eliminate the first term of Eq. 4.14. As there is mainly ground mode contribution involved in the $2^{\text {nd }}$ domain i.e. CPMG pulse train, the relative intensity 
$I_{0,0}=\int \varphi_{2}^{0} d V \cdot \int \varphi_{2}^{0} \varphi_{1}^{0} d V \cdot \int \sin \Phi \varphi_{1}^{0} d V$. According to Eq. 4.8, the relative intensity $I_{0}$ in the 1D signal pulse sequence shown in Section 4.1.1 can be estimated as $I_{0}=\int \varphi_{1}^{0} d V \cdot \int \sin \Phi \varphi_{1}^{0} d V$. With an assumption that the fast diffusion limits can be satisfied for the sample, the eigenfunction $\varphi_{2}^{0}$ for the ground $T_{2}$ mode would be constant $[158,163]$, resulting to the equivalent values for $I_{0,0}$ and $I_{0}$. As a result, $R\left(t_{\text {diff }}, N T_{\mathrm{E}}\right)$ multiplied by $I_{0}$ can be subtracted from $E\left(t_{\mathrm{diff}}, N T_{\mathrm{E}}\right)$ (similar to the $1 \mathrm{D}$ case), giving a $2 \mathrm{D}$ data set characterized by the first $T_{1}$ mode and ground $T_{2}$ mode:

$$
\begin{aligned}
S\left(t_{\text {diff }}, N T_{\mathrm{E}}\right) & =m_{0} \cdot I_{1,0} \exp \left(-\frac{t_{\text {diff }}}{\tau_{1}^{1}}\right) \exp \left(-\frac{N T_{\mathrm{E}}}{\tau_{2}^{0}}\right) \\
& =m_{0} \cdot I_{1,0} \exp \left(-\frac{4 t_{\text {diff }} D \zeta_{1}^{2}}{a^{2}}\right) \exp \left(-\frac{N T_{\mathrm{E}}}{\tau_{2}^{0}}\right)
\end{aligned}
$$

Similarly to the $1 \mathrm{D}$ case, the signal from a rock sample with a distribution of pore lengths $a$ will be the sum of the contributions from all pores in the system. As a result, Eq. 4.16 can be rewritten as:

$$
\begin{aligned}
S\left(t_{\text {diff }}, N T_{\mathrm{E}}\right)=I_{1,0} \iint F\left(a, T_{2}\right) \exp \left(-\frac{4 t_{\mathrm{diff}} D \zeta_{1}^{2}}{a^{2}}\right) \\
\quad \exp \left(-\frac{N T_{\mathrm{E}}}{T_{2}}\right) d a d T_{2} .
\end{aligned}
$$

Since the relaxation effect during the $2^{\text {nd }}$ domain of the pulse sequence is mainly contributed from the ground $T_{2}$ mode, the high and groundeigenmode correlation function can be obtained using 2D numerical inversion algorithm [95] and rescaled to pore length-relaxation correlation function $F\left(a, T_{2}\right)$ via $a \approx 2 \zeta_{1} \sqrt{D \tau_{1}^{1}}$.

\subsubsection{Correlation of ground $T_{1}$ and first $T_{1}$ mode}

Similarly, the correlation of the ground and the first excited $T_{1}$ mode will provide the information of pore length, $a$ and pore surface relaxivity, 
$\rho_{1}$ simultaneously if the detection of these two eigenmodes are combined in a $2 \mathrm{D}$ experiment. To achieve this, a small tip angle pulse train will be complemented after the aforementioned 1D signal pulse sequences (shown in Fig. 4.4). During the $2^{\text {nd }}$ domain of this pulse sequence, the evolution of the magnetization aligning along $z$ axis can be recorded by acquiring the amplitude of FID using the small tip angle pulse train [32]. The phase cycling of this 2D pulse sequence is shown in Table. 4.4. $\phi_{1}, \phi_{2}, \phi_{3}$ and $\phi_{4}, \phi_{\alpha}$ and $\phi_{A c q}$ represent the phase of the first four $\pi / 2$ pulses, small tip angle and the acquisition, respectively. Using the magnetization vector expression given in Eq. 2.12, the FID intensity after the $N$-th $\alpha$ pulse in the $2^{\text {nd }}$ domain of the $2 \mathrm{D}$ pulse sequence can be expressed as a recursion expression:

$$
\begin{aligned}
M_{N}^{x y} & =\left[M_{(N-1)}^{z} \exp \left(-\frac{t_{\mathrm{a}}}{T_{1}}\right)+M_{0}\left(1-\exp \left(-\frac{t_{\mathrm{a}}}{T_{1}}\right)\right)\right] \sin \alpha \\
M_{N}^{z} & =\left[M_{(N-1)}^{z} \exp \left(-\frac{t_{\mathrm{a}}}{T_{1}}\right)+M_{0}\left(1-\exp \left(-\frac{t_{\mathrm{a}}}{T_{1}}\right)\right)\right] \cos \alpha
\end{aligned}
$$

where $N$ represents the number of the small tip angle pulses and $t_{\mathrm{a}}$ is the signal evolution time between the adjacent FID acquisition. $M_{N}^{x y}$ and $M_{N}^{z}$ stand for the magnetization components in the transverse plane and along the longitudinal axis, respectively. For an initial condition, $M_{1}^{x y}=M_{0} \sin \alpha$ and $M_{1}^{z}=M_{0} \cos \alpha$. Therefore, by using the phase cycling suggested in Table. 4.4, the magnetization recovery term $M_{0}\left(1-\exp \left(-\frac{t_{\mathrm{a}}}{T_{1}}\right)\right)$ in Eq. 4.18 can be cancelled, leaving a decay term that expressed as:

$$
M\left((N-1) t_{\mathrm{a}}\right)=2 M_{0} \sin \alpha \cdot \exp \left[-\frac{(N-1) t_{\mathrm{a}}}{\tau_{1}}\right] \cdot \cos ^{N-1} \alpha
$$

As a result, according to Eq. 4.12, the acquired data using the 2D pulse 
sequence can be expressed as:

$$
\begin{aligned}
E\left(t_{\text {diff }},(N-1) t_{\mathrm{a}}\right) & =\int m\left(\mathbf{r}, t_{\text {diff }},(N-1) t_{\mathrm{a}}\right) d V \\
& =m_{0} \cdot \sum_{m, n} I_{m, n} \exp \left(-\frac{t_{\text {diff }}}{\tau_{1}^{m}}\right) \exp \left[-\frac{(N-1) t_{\mathrm{a}}}{\tau_{1}^{n}}\right] \cos ^{N-1} \alpha \cdot \sin \alpha,
\end{aligned}
$$

where $\tau_{1}^{m}$ and $\tau_{1}^{n}$ are the decay times of $m$-th and $n$-th $T_{1}$ mode, and $I_{m, n}$ is the correlation intensity of the eigenmodes. The degree of $\alpha$ corresponds to the tip angle of the pulses in the $2^{\text {nd }}$ domain.

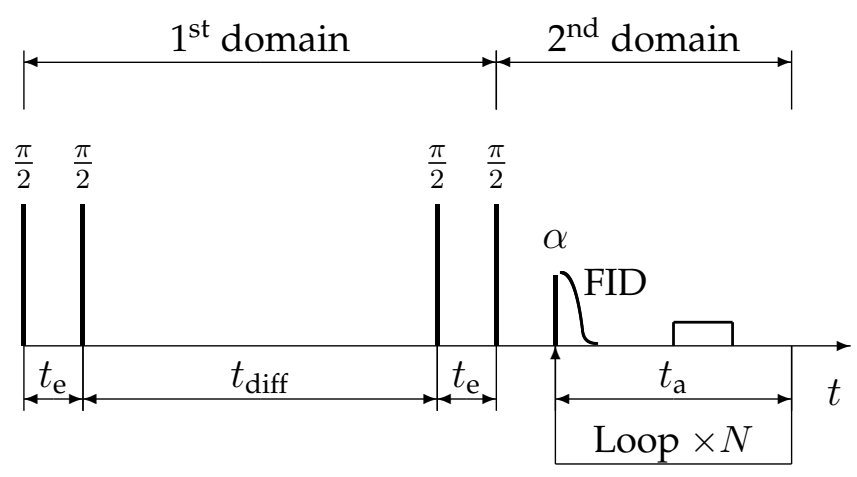

FIG. 4.4: 2D NMR pulse sequence used to correlate the ground and first excited eigenmode. $t_{\mathrm{e}}$ is the encoding period and $t_{\text {diff }}$ is the observation time of molecular diffusion. $t_{\mathrm{a}}$ is the period for the acquisition of FID signal. The $\alpha$ pulses have the same duration of $\pi / 2$ and $\pi$ pulses and the adjusted pulse power. The homospoil gradients represented by the rectangles were used to cancel the unwanted coherence pathway in the acquired signal. The phase cycling used in this pulse sequence is given in Tab. 4.4.

As discussed in Section 4.1.1, by adopting the strategy from 1D case in the $1^{\text {st }}$ domain of the $2 \mathrm{D}$ pulse sequence, the information from the ground and first $T_{1}$ mode can be both encoded. At the end of the $1^{\text {st }}$ domain, the magnetization is refocused and rotated back along the longitudinal axis, leading to a situation where the ground $T_{1}$ mode dominates the second stage of the $2 \mathrm{D}$ pulse sequence. As a result, the acquired data from $2 \mathrm{D}$ 
Table 4.4: Phase cycling for $2 \mathrm{D}$ ground and first $T_{1}$ mode correlation pulse sequence. $\phi_{1}, \phi_{2}, \phi_{3}$ and $\phi_{4}, \phi_{\alpha}$ and $\phi_{A c q}$ represent the phase of the first four $\pi / 2$ pulses, small tip angle and the acquisition, respectively.

\begin{tabular}{cccccc}
\hline$\phi_{1}$ & $\phi_{2}$ & $\phi_{3}$ & $\phi_{4}$ & $\phi_{\alpha}$ & $\phi_{A c q}$ \\
\hline$\pi / 2$ & 0 & 0 & $\pi$ & $\pi / 2$ & $\pi / 2$ \\
$3 \pi / 2$ & 0 & 0 & $\pi$ & $\pi / 2$ & $3 \pi / 2$ \\
$\pi / 2$ & $\pi$ & 0 & $\pi$ & $\pi / 2$ & $3 \pi / 2$ \\
$3 \pi / 2$ & $\pi$ & 0 & $\pi$ & $\pi / 2$ & $\pi / 2$ \\
$\pi / 2$ & 0 & 0 & 0 & $\pi / 2$ & $\pi / 2$ \\
$3 \pi / 2$ & 0 & 0 & 0 & $\pi / 2$ & $3 \pi / 2$ \\
$\pi / 2$ & $\pi$ & 0 & 0 & $\pi / 2$ & $3 \pi / 2$ \\
$3 \pi / 2$ & $\pi$ & 0 & 0 & $\pi / 2$ & $\pi / 2$ \\
\hline
\end{tabular}

pulse sequence can be further approximately expressed as:

$E\left(t_{\text {diff }},(N-1) t_{\mathrm{a}}\right) \approx m_{0} \cdot \cos ^{N-1} \alpha \cdot \sin \alpha$
$\cdot\left[I_{0,0} \exp \left(-\frac{t_{\text {diff }}}{\tau_{1}^{0}}\right) \exp \left[-\frac{(N-1) t_{\mathrm{a}}}{\tau_{1}^{0}}\right]+I_{1,0} \exp \left(-\frac{t_{\text {diff }}}{\tau_{1}^{1}}\right) \exp \left[-\frac{(N-1) t_{\mathrm{a}}}{\tau_{1}^{0}}\right]\right]$.

As seen from Eq. 4.21, the first term in the right bracket is the contribution from self-correlation of the ground $T_{1}$ mode, and the second term represents the signal experiencing the first and ground $T_{1}$ eigenmodes correlation, the detection of which is at the heart of this part of the work. As can be expected, the distribution of the first term will lay along the diagonal line of $T_{1}-T_{1}$ correlation map and the second term will appear away from the diagonal line.

To extract the second term in Eq. 4.21, the ground $T_{1}$ contribution in the $1^{\text {st }}$ domain needs to be calibrated out. It is notable that the ground $T_{1}$ eigenmode is already detected in the $2^{\text {nd }}$ domain of this technique, which exactly provides a means to obtain the weight of ground $T_{1}$ mode and isolate the first $T_{1}$ mode contribution in the $1^{\text {st }}$ domain. The detailed steps to obtain the first and ground $T_{1}$ eigenmodes correlation contribution are 
given as follows:

1: Calibrating the amplitudes of 2D signal $E\left(t_{\text {diff }},(N-1) t_{\mathrm{a}}\right)$ in Eq. 4.21 by $\cos ^{N-1} \alpha \cdot \sin \alpha$ first.

2: Processing the calibrated $E\left(t_{\text {diff }},(N-1) t_{\mathrm{a}}\right)$ by using 2D ILT and obtaining the correlation distribution $F\left(\tau^{(1 \mathrm{st})}, \tau^{(2 \mathrm{nd})}\right)$. This obtained function is actually $F\left(\left[\tau_{0}, \tau_{1}\right], \tau_{0}\right)$.

3: Evaluating the relative intensities of the ground $T_{1}$ mode $I_{0,0}$ in order to eliminate it from the higher modes in the $1^{\text {st }}$ domain.

4: Obtaining a data matrix $S\left(t_{\text {diff }},(N-1) t_{\mathrm{a}}\right)$ by compensating the groundground $T_{1}$ eigenmode correlation contribution in Eq. 4.21 and can thus be expressed as:

$$
\begin{aligned}
S\left(t_{\text {diff }},(N-1) t_{\mathrm{a}}\right) & =m_{0} \cdot I_{1,0} \exp \left(-\frac{t_{\text {diff }}}{\tau_{1}^{1}}\right) \exp \left[-\frac{(N-1) t_{\mathrm{a}}}{\tau_{1}^{0}}\right] \\
& =m_{0} \cdot I_{1,0} \exp \left(-\frac{4 t_{\text {diff }} D \zeta_{1}^{2}}{a^{2}}\right) \exp \left[-\frac{(N-1) t_{\mathrm{a}}}{T_{1}}\right]
\end{aligned}
$$

For a porous system, the signal with a distribution of pore lengths $a$ will be the sum of the contributions from all pores in the system. As a result, Eq. 4.22 can be rewritten as

$$
\begin{aligned}
& S\left(t_{\text {diff }},(N-1) t_{\mathrm{a}}\right)=I_{1,0} \iint F\left(a, T_{1}\right) \exp \left(-\frac{-4 t_{\text {diff }} D \zeta_{1}^{2}}{a^{2}}\right) \\
& \exp \left[-\frac{(N-1) t_{\mathrm{a}}}{T_{1}}\right] d a d T_{1}
\end{aligned}
$$

which allows one to obtain the pore length-relaxation correlation function $F\left(a, T_{1}\right)$ using a 2D ILT algorithm.

In this experiment, the eigenmodes excited by this pulse sequence are only from longitudinal relaxation $T_{1}$, which leads to two advantages of this 
strategy. Firstly, the magnetization was restored along the longitudinal axis after the fourth $90^{\circ}$ pulse, which indicates a re-established uniform magnetization profile along the pore space. Therefore, only the ground $T_{1}$ mode will be dominantly excited during the $2^{\text {nd }}$ domain of signal acquisition and the detected signal can be employed to isolate the contribution from the ground $T_{1}$ mode in the $1^{\text {st }}$ encoding period. By this means, the correlation distribution of high- and ground $T_{1}$ mode can be obtained with no needs of the reference pulse sequence as compared to the correlation experiment of ground $T_{2}$ and first $T_{1}$ mode. Secondly, the intensity of $2 \mathrm{D}$ spectrum in this method can be guaranteed non-negative because of the orthogonality of the eigenmodes involved during the successive time domains, and therefore it naturally avoids the misinterpretation from the non-negative restrains during 2D ILT [227].

Independently, in order to validate the eigenmodes detected in the pulse sequence given in Fig. 4.4, an extra 2D pulse sequence is utilized to couple only the ground $T_{1}$ mode in two successive dimensions. The pulse sequence is shown in Fig. 4.5 and its phase cycling given in Table. 4.5.

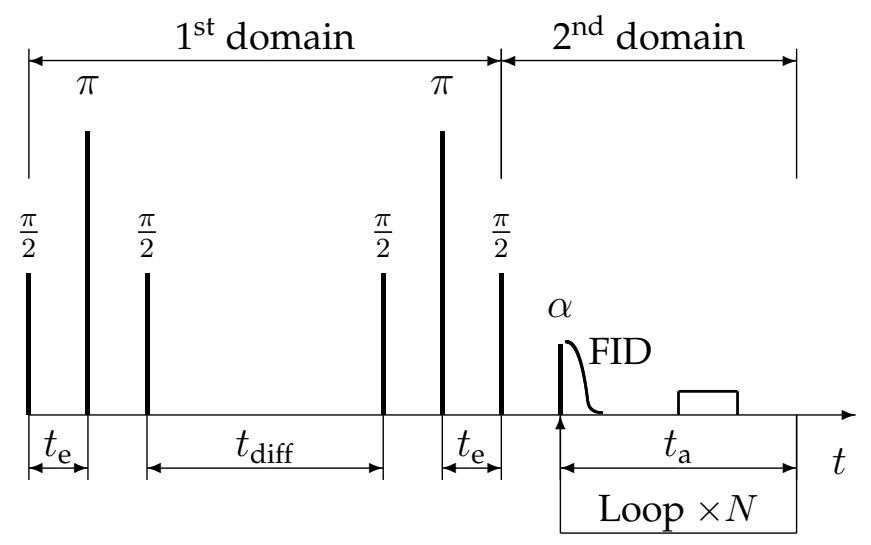

FIG. 4.5: $2 \mathrm{D}$ NMR pulse sequence used to validate the ground and first excited $T_{1}$ mode correlation experiments. Two extra $\pi$ pulses are inserted in the $1^{\text {st }}$ domain. The phase cycling used in this pulse sequence is given in Tab. 4.5.

Similarly to 1D reference pulse sequence shown in the bottom of Fig. 4.2, a $\pi$ pulse is inserted in the middle of the first two $\pi / 2$ pulse, to compensate 
Table 4.5: Phase cycling for validating $2 \mathrm{D}$ ground and first $T_{1}$ mode correlation pulse sequence. $\phi_{1}, \phi_{2}, \phi_{3}$ and $\phi_{4}, \phi_{5}, \phi_{\alpha}$ and $\phi_{A c q}$ are the phase of the first four $\pi / 2$ pulses, $\pi$ pulse, small tip angle pulse, and the acquisition, respectively.

\begin{tabular}{ccccccc}
\hline$\phi_{1}$ & $\phi_{2}$ & $\phi_{3}$ & $\phi_{4}$ & $\phi_{5}$ & $\phi_{\alpha}$ & $\phi_{A c q}$ \\
\hline 0 & 0 & 0 & $\pi$ & $\pi / 2$ & $\pi / 2$ & 0 \\
$\pi$ & 0 & 0 & $\pi$ & $\pi / 2$ & $\pi / 2$ & $\pi$ \\
0 & $\pi$ & 0 & $\pi$ & $\pi / 2$ & $\pi / 2$ & 0 \\
$\pi$ & $\pi$ & 0 & $\pi$ & $\pi / 2$ & $\pi / 2$ & $\pi$ \\
0 & 0 & 0 & $\pi$ & $\pi / 2$ & $\pi / 2$ & 0 \\
$\pi$ & 0 & 0 & $\pi$ & $\pi / 2$ & $\pi / 2$ & $\pi$ \\
0 & $\pi$ & 0 & $\pi$ & $\pi / 2$ & $\pi / 2$ & 0 \\
$\pi$ & $\pi$ & 0 & $\pi$ & $\pi / 2$ & $\pi / 2$ & $\pi$ \\
\hline
\end{tabular}

the dephasing effect due to the induced magnetic fields. As a result, only the ground $T_{1}$ mode is detected in the $1^{\text {st }}$ domain of this $2 \mathrm{D}$ experiments. By applying the small tip angle train in the $2^{\text {nd }}$ domain, the ground $T_{1}$ mode is obtained again and the required 2D data can be expressed as:

$$
S\left(t_{\text {diff }},(N-1) t_{\mathrm{a}}\right)=m_{0} \cdot \exp \left(-\frac{t_{\text {diff }}}{\tau_{1}^{0}}\right) \exp \left[-\frac{(N-1) t_{\mathrm{a}}}{\tau_{1}^{0}}\right] \text {. }
$$

\subsection{Estimation of surface relaxivity}

Surface relaxivity $\rho$ reflects the interaction strength of fluid nuclear spins and pore surfaces. A typical approach to evaluate the surface relaxivity of rock samples in NMR is matching the $1 \mathrm{D}$ relaxation time distribution with the pore length scales [166-170]. Another approach is performing the Padé approximation on the calculated $D\left(T_{2}\right)$ curve from 2D $D-T_{2}$ correlation map [228, 229]. As discussed in Eq. 3.6, the apparent diffusion coefficient of fluid molecules $D_{a}$ in porous media will attenuate from its self diffusion coefficient $D_{0}$ because of the restriction of the pore boundary [49]. Therefore, within a certain observation time $\Delta$ of molecular diffusion, $D_{a}$ will decrease 
more from $D_{0}$ in the smaller pore whereas is close to $D_{0}$ in the larger pore. Since $T_{2}$ is a pore length related parameter, the relationship of $D\left(T_{2}\right)$ in different pore regimes can be obtained from $D-T_{2}$ correlation maps using the substitution of $T_{2}$ values to the Padé approximation of $D_{a}$ used in Eq. 3.6:

$$
\frac{D\left(T_{2}\right)}{D_{0}} \approx 1-\gamma \frac{\delta}{\delta+\gamma}
$$

where $D\left(T_{2}\right)$ is the logarithmic mean value of $D$ in each $T_{2}$ value. $\delta=$ $\frac{4 \sqrt{D_{0} \Delta}}{9 \sqrt{\pi} T_{2 s} \rho_{2}}$, which is a value reflecting the short-time behavior of $D_{a} . T_{2 \mathrm{~s}}$ is the surface relaxation contribution of $T_{2} \cdot \gamma=1-\frac{1}{F \phi}$, which is a factor related to the tortuosity of the rock samples. As seen from Eq. 4.25, the surface relaxivity $\rho_{2}$ can be easily extracted from the obtained $D\left(T_{2}\right)$ curve with the pre-knowledge of the formation factor $F$ and porosity $\phi$ of rock samples.

\subsubsection{Estimation of surface relaxivity $\rho_{2}$}

Surface relaxivity $\rho_{2}$ can be estimated based on the correlation function of pore length and relaxation time $F\left(a, T_{2}\right)$ acquired in Section 4.2.1. With the assumption of spherical pore shape, the relationship between transverse relaxation time $T_{2}$ and pore length (or pore diameter) $a$ can be derived from Eq. 3.5 as:

$$
T_{2} \approx \frac{1}{6 \rho_{2}} a
$$

The relationship $T_{2}(a)$ can be easily obtained from the $2 \mathrm{D}$ correlation function $F\left(a, T_{2}\right)$ and the relaxation time $T_{2}$ can be reinterpreted as a function of $a$. Thus the horizontal-to-vertical ratios of the correlation function $T_{2}(a)$ along the $a$ axes can be calculated and the results allow one to extract the effective surface relaxivity $\rho_{2}$ of rock cores. Since the relaxation time $T_{2}$ is not strongly affected by the diffusion in internal magnetic field gradients, the relationship of Eq. 4.26 is more suitable in the low-field although the signal-to-noise is lower compared to the high-field. 


\subsubsection{Estimation of surface relaxivity $\rho_{1}$}

Similarly, the surface relaxivity $\rho_{1}$ will be estimated based on the correlation function of pore length and relaxation time $F\left(a, T_{1}\right)$ acquired in Section 4.2.2. Again, the relationship between longitudinal relaxation time $T_{1}$ and pore length (or pore diameter) $a$ can be derived from Eq. 3.5 as:

$$
T_{1} \approx \frac{1}{6 \rho_{1}} a .
$$

The relationship $T_{1}(a)$ can be obtained from 2D correlation function $F\left(a, T_{1}\right)$ and the effective surface relaxivity $\rho_{1}$ of rock cores can be estimated from $T_{1}(a)$.

\subsection{Experimental}

Two rock samples with different lithologies (Parker sandstone: porosity, $16.4 \%$; gas permeability, $30 \mathrm{mD}$ and Winterset limestone: porosity, $24.4 \%$; gas permeability, $15 \mathrm{mD}$ ) were studied in the $T_{1}-T_{2}$ eigenmode correlation experiment. The 1D experiments were performed on a $2 \mathrm{MHz}$ Rock Core Analyser (Magritek Ltd, Wellington, NZ). Both rock samples had the external dimensions of $38 \mathrm{~mm}$ diameter and $63 \mathrm{~mm}$ length. The RF pulse length was set to be $25 \mu \mathrm{s}$. The encoding period $t_{\mathrm{e}}$ was $1.5 \mathrm{~ms}$ in Parker sandstone and $9 \mathrm{~ms}$ in Winterset limestone. The observation time $t_{\text {diff }}$ varied from $0.5 \mathrm{~ms}$ to $3000 \mathrm{~ms}$ in Parker sandstone and to $6000 \mathrm{~ms}$ in Winterset limestone in 50 steps logarithmically. To validate the results of pore length scale, the 1D experiments of these two rock cores were performed on a prototype $64 \mathrm{MHz}$ cryogen free NMR Imaging system (Cryogenic Ltd, London, UK) with homebuilt gradients and RF coil operated by a Kea console (Magritek Ltd, Wellington, NZ). The encoding period $t_{\mathrm{e}}$ was chosen to be $400 \mu \mathrm{s}$ in both rock cores. The broadening of the NMR line-width due to the internal magnetic field gradients alone can be extracted from the decay rates of the FID signals [230], which resulted in $13 \mathrm{~Hz}$ at $2 \mathrm{MHz}$ 
and $334 \mathrm{~Hz}$ at $64 \mathrm{MHz}$ for sandstone and $8 \mathrm{~Hz}$ at $2 \mathrm{MHz}$ and $207 \mathrm{~Hz}$ at $64 \mathrm{MHz}$ for limestone. These line-width combined with the corresponding encoding period $t_{\mathrm{e}}$ satisfy the weak encoding condition $\gamma \Delta B_{i n} t_{\mathrm{e}}<1$ as discussed in section 4.1.1. $T_{1}-T_{2}$ eigenmode correlation experiments were performed with the same parameters for the 1D stimulated echo sequence and followed by a CPMG pulse train on the $2 \mathrm{MHz}$ Rock Core Analyzer. The echo spacing $T_{\mathrm{E}}$ was $125 \mu$ s and echo number $N$ was 6400 in sandstone and 24000 in limestone. Each data set was averaged 64 times for both 1D and $2 \mathrm{D}$ cases, resulting in a total acquisition time of $5 \mathrm{hrs}$.

Two rock samples (Nugget sandstone: porosity, 10.5\%; gas permeability, $3 \mathrm{mD}$ and Winterset limestone: porosity, 24.4\%; gas permeability, $15 \mathrm{mD}$ ) were adapted in the $T_{1}-T_{1}$ eigenmode correlation experiment on a prototype $64 \mathrm{MHz}$ cryogen free NMR Imaging system (Cryogenic Ltd, London, UK) with homebuilt gradients and RF coils operated by a Kea console (Magritek Ltd, Wellington, NZ). The RF pulse length was set to be $60 \mu \mathrm{s}$. The encoding period $t_{\mathrm{e}}$ was $400 \mu \mathrm{s}$ in both rock cores. The observation time $t_{\text {diff }}$ varied from $0.5 \mathrm{~ms}$ to $3000 \mathrm{~ms}$ in sandstone and to $6000 \mathrm{~ms}$ in limestone in 50 steps logarithmically. The small tip angle $\alpha$ is modified to be $8^{\circ}$. The acquisition time $t_{\mathrm{a}}$ was $6.4 \mathrm{~ms}$ in Nugget sandstone and $20.48 \mathrm{~ms}$ in Winterset limestone. The number of FID was fixed to be 120 in both cores.

\subsection{Results}

\subsubsection{D experimental results}

Before presenting the 2D eigenmode correlation experimental results, we prefer to show the $1 \mathrm{D}$ pore length scales of the rock cores used in this work first.

The 1D experimental data of Parker sandstone is illustrated in Fig. 4.6 (a). It can be seen from the data plot that the magnitude from the 1D signal pulse sequence (diamond) rapidly attenuates for short $t_{\text {diff }}$ values, compare 
to reference pulse sequence (triangle), however both data sets appear to decay at the same rates at the long $t_{\text {diff. }}$ This shows that the high and ground $T_{1}$ mode are dominant at the beginning of the observation time and only the ground $T_{1}$ mode survives at long $t_{\text {diff }}$ for the $1 \mathrm{D}$ signal pulse sequence. The relative intensity of the ground mode in Eq. 4.7 can then be calculated using the ratio of these two data sets at long $t_{\text {diff, }}$, i.e. $I_{0}=\frac{1}{1.45} \approx 0.69$. An extra data set with $t_{\mathrm{e}}=400 \mu$ s (circle in Fig. 4.6 (a)) was acquired using the 1D signal pulse sequence in Fig. 4.2, decaying at approximately the same rates as the reference data. This proves that the high $T_{1}$ mode with a short encoding time $t_{\mathrm{e}}$ is too weak to be detected at low-field.

To extract the pore length scales from Parker sandstone, the reference data is first multiplied by $I_{0}$ giving us the "normalized" reference data set which is then subtracted from the the 1D signal data, leaving us with a $1 D$ data $S\left(t_{\text {diff }}\right)$ shown in the inset of Fig. 4.6 (a). Then 1D inversion algorithm was used to obtain decay time $\tau_{1}^{1}$ distribution from $S\left(t_{\text {diff }}\right)$, which directly yields us the pore length distribution according to $a \approx 2 \zeta_{1} \sqrt{D \tau_{1}^{1}}$.

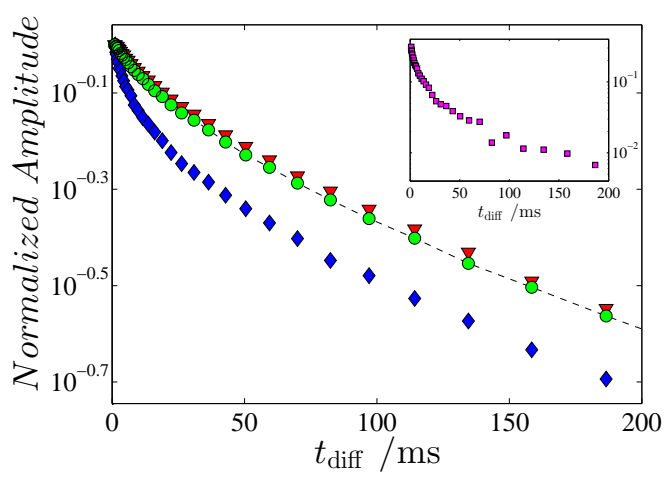

(a)

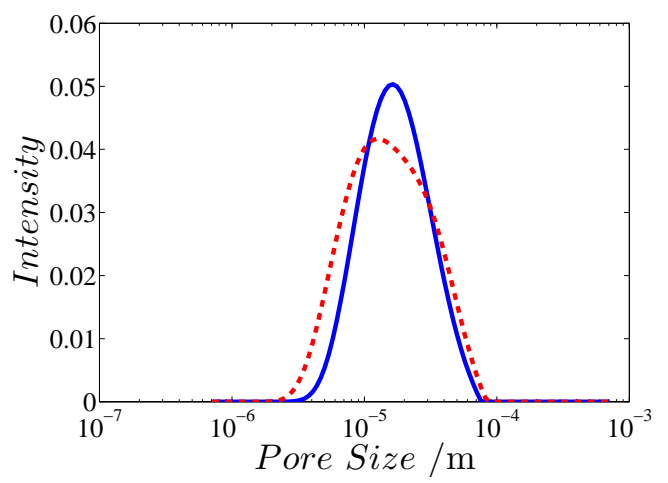

(b)

FIG. 4.6: (a) The normalized 1D experimental data of Parker sandstone. The triangle and diamond markers represent the amplitudes from 1D reference and signal pulse sequences, respectively. The circles represent the amplitudes from 1D signal pulse sequence with $t_{\mathrm{e}}=400 \mu \mathrm{s}$. The right inset gives the result of $S\left(t_{\mathrm{diff}}\right)$; (b) The pore length scales of Parker sandstone determined at $2 \mathrm{MHz}$ (solid lines) and $64 \mathrm{MHz}$ (dashed lines), respectively.

As illustrated in Fig. 4.6 (b), the pore length distributions of sandstone 
from $2 \mathrm{MHz}$ and $64 \mathrm{MHz}$ show good correspondence, which demonstrates that the method of using high eigenmode to determine pore length scales is feasible even at $2 \mathrm{MHz}$. The relative pore length distribution expands less than one and a half orders of magnitude with a logarithmic mean value of around $18.9 \mu \mathrm{m}$. Some regions with small pore length were not resolved at $2 \mathrm{MHz}$, which is ascribed to the longer $t_{\mathrm{e}}$ used in low-field experiments. During this period, the molecules diffuse over the local pore length and the non-uniform magnetization profile is averaged out in those pores.

The 1D experimental data of Winterset limestone is shown in Fig. 4.7 (a). The data from 1D signal and reference pulse sequences exhibit similar decay pattern compared to the sandstone results and the relative intensity of the limestone $I_{0}=\frac{1}{1.35} \approx 0.74$. Again, the extra data set with $t_{\mathrm{e}}=400 \mu \mathrm{s}$ (circle in Fig. 4.7 (a)) attenuates at approximately the same rates as the reference data and proves that the first $T_{1}$ mode with a shorter encoding time $t_{\mathrm{e}}$ is too weak to be detected.

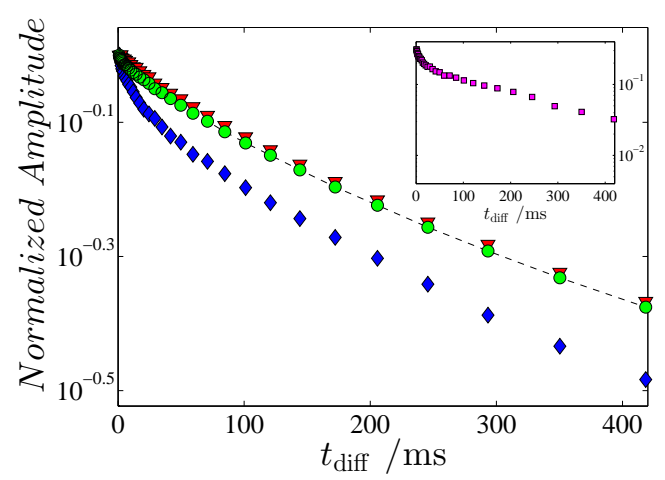

(a)

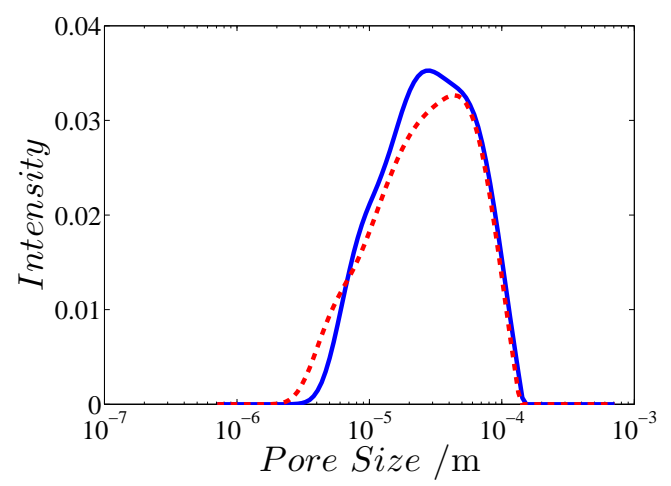

(b)

FIG. 4.7: (a) The normalized 1D experimental data of Winterset limestone. The triangle and diamond markers represent the amplitudes from 1D reference and signal pulse sequences, respectively. The circles represent the amplitudes from $1 \mathrm{D}$ signal pulse sequence with $t_{\mathrm{e}}=400 \mu \mathrm{s}$. The right inset gives the result of $S\left(t_{\text {diff }}\right) ;(\mathrm{b})$ The pore length scales of Winterset limestone determined at $2 \mathrm{MHz}$ (solid lines) and $64 \mathrm{MHz}$ (dashed lines), respectively.

The extracted pore length distributions of Winterset limestone from 1D data $S\left(t_{\text {diff }}\right)$ are shown in Fig. 4.7 (b) and correspond well at $2 \mathrm{MHz}$ and 
$64 \mathrm{MHz}$. The relative pore length distribution extends almost two orders of magnitude with the logarithmic mean pore length of $40.9 \mu \mathrm{m}$. Thus, from the comparison of these features in two rock samples, a conclusion can be drawn that there are bigger pores and more heterogeneous pore network in Winterset limestone than that in Parker sandstone.

Moreover, the 1D experimental data of Nugget sandstone at $64 \mathrm{MHz}$ is illustrated in Fig. 4.8 (a). It can be seen from the data plot that the magnitude from the 1D signal pulse sequence (diamond) rapidly attenuates for short $t_{\text {diff }}$ values, compare to reference pulse sequence (triangle), however both data sets appear to decay at the same rates at the long $t_{\text {diff. }}$ The relative intensity of the ground mode in Eq. 4.7 can then be calculated using the ratio of these two data sets at long $t_{\text {diff, }}$, i.e. $I_{0}=\frac{1}{1.18} \approx 0.85$.

Subsequently, the reference data is first multiplied by $I_{0}$ giving us the "normalized" reference data set which is then subtracted from the the 1D signal data, leaving us with a 1D data $S\left(t_{\text {diff }}\right)$ shown in the inset of Fig. 4.8 (a). Then 1D inversion algorithm was used to obtain decay time $\tau_{1}^{1}$ distribution from $S\left(t_{\text {diff }}\right)$, which directly yields us the pore length distribution according to $a \approx 2 \zeta_{1} \sqrt{D \tau_{1}^{1}}$. As illustrated in Fig. $4.8(\mathrm{~b})$, the pore length distribution expands around one order of magnitude with a logarithmic mean value of around $10.3 \mu \mathrm{m}$.

\subsection{2 $T_{1}-T_{2}$ eigenmode correlation results}

\section{D correlation results}

The 2D experimental data $E\left(t_{\text {diff }}, N T_{\mathrm{E}}\right)$ and $R\left(t_{\text {diff }}, N T_{\mathrm{E}}\right)$ of the sandstone were acquired using the pulse sequences shown in Fig. 4.3. In order to isolate the contribution from ground eigenmodes correlation from the first term of Eq. 4.14, the value of $I_{0,0}$ should be determined. As discussed in Section. 4.2.1, $I_{0,0}$ would be approximately the same as $I_{0}$ in the $1 \mathrm{D}$ experiment, assuming that the contribution from ground eigenmode is predominant during the CPMG acquisition. To validate this, the first column 


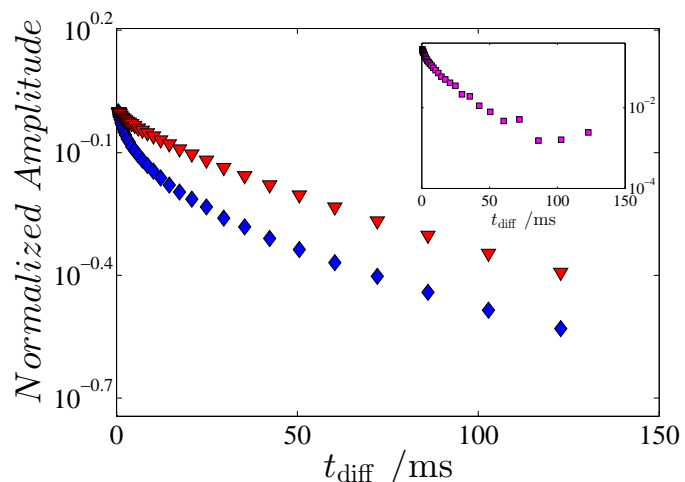

(a)

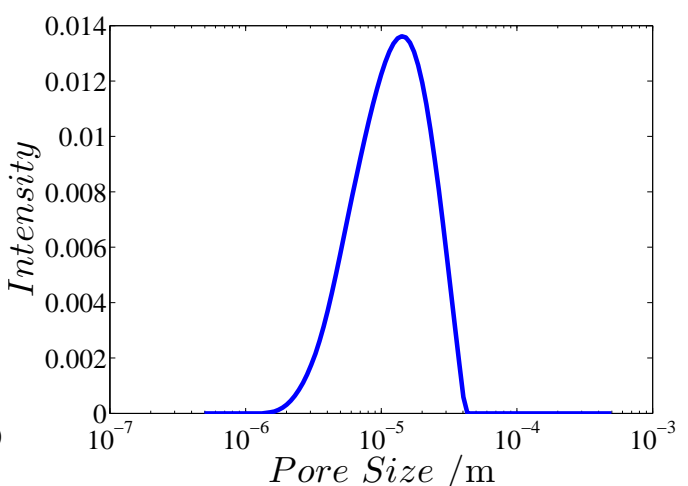

(b)

FIG. 4.8: (a) The normalized 1D experimental data of Nugget sandstone. The triangle and diamond markers represent the amplitudes from 1D reference and signal pulse sequences, respectively. The right inset gives the result of $S\left(t_{\text {diff }}\right)$; (b) The determined pore length scales of Nugget sandstone.

from the data sets of the 2D signal and reference pulse sequences are shown in Fig. 4.9 (a) and the value of $I_{0,0}$ was estimated to be 0.68 , which is close to $I_{0}(\approx 0.69)$ in $1 \mathrm{D}$ experiment. Subsequently, $E\left(t_{\text {diff }}, N T_{\mathrm{E}}\right)$ was subtracted from "normalized" $I_{0,0} \cdot R\left(t_{\text {diff }}, N T_{\mathrm{E}}\right)$, leaving a $2 \mathrm{D}$ data set $S\left(t_{\text {diff }}, N T_{\mathrm{E}}\right)$ as shown in Fig. 4.9 (b). The $2 \mathrm{D}$ data decays dramatically along $t_{\text {diff }}$ axis while attenuating gradually along the CPMG acquisition, which suggests that a comparably higher mode is being contributed to $S\left(t_{\text {diff }}, N T_{\mathrm{E}}\right)$ along $t_{\text {diff }}$ axis.

The correlation distribution of 2D eigenmodes $F\left(\tau_{1}^{1}, \tau_{2}^{0}\right)$ was acquired from $S\left(t_{\text {diff }}, N T_{\mathrm{E}}\right)$ using 2D numerical inversion algorithm. Meanwhile, the ground modes correlation function $F\left(\tau_{1}^{0}, \tau_{2}^{0}\right)$ was obtained from 2D reference data $R\left(t_{\text {diff }}, N T_{\mathrm{E}}\right)$ as a comparison. The results of these two correlation functions are shown in Fig. 4.10. The color scales are identical to compare the intensities of these 2D correlation maps, while the 1D projected curves are normalized in order to examine the coincidence of $T_{2}$ distributions in a proper way. It is worth noting that a larger optimized regularization factor should be chosen during the processing of $S\left(t_{\text {diff }}, N T_{\mathrm{E}}\right)$ because of the poorer signal-to-noise ratio compared to $R\left(t_{\text {diff }}, N T_{\mathrm{E}}\right)$. 

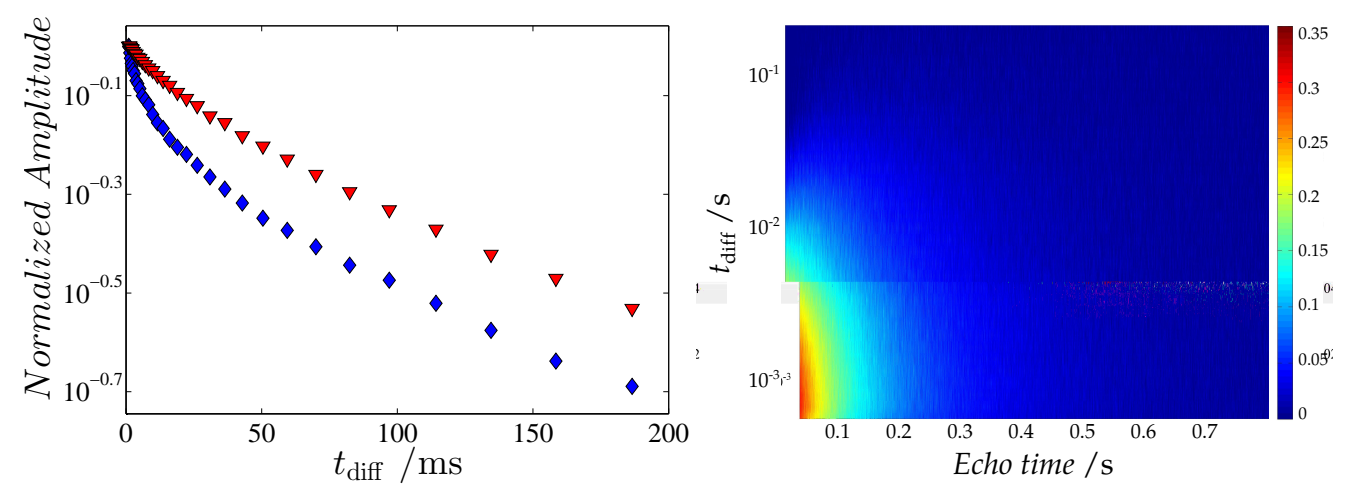

(a)

(b)

FIG. 4.9: (a) The first column from the data matrices of 2D signal pulse sequence (diamond) and 2D reference pulse sequence (triangle); (b) the data set $S\left(t_{\mathrm{diff}}, N T_{\mathrm{E}}\right)$ in the sandstone at $2 \mathrm{MHz}$. To better observe the amplitude attenuation along the observation time $t_{\text {diff, }}$, the logarithmic scale is used and only the time range from $0.5 \mathrm{~ms}$ to $187 \mathrm{~ms}$ was shown.

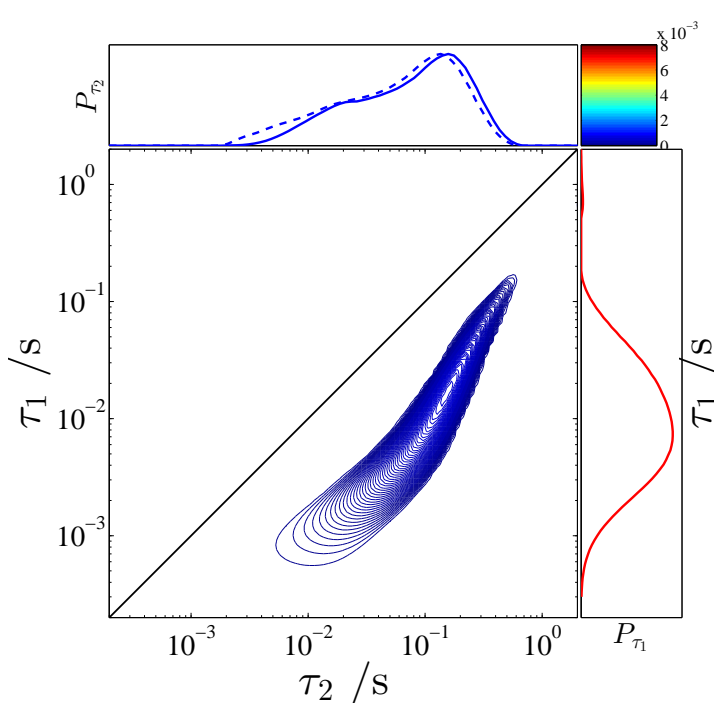

(a)

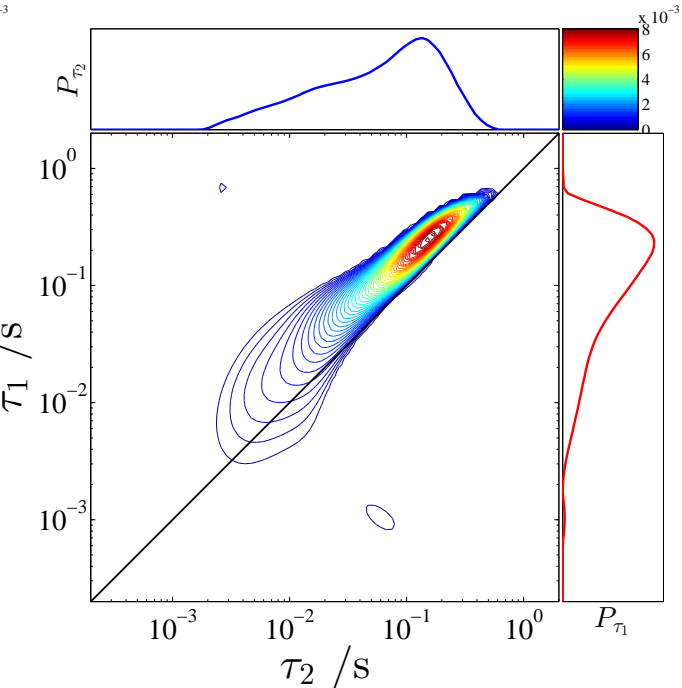

(b)

FIG. 4.10: Comparison of the distribution functions (a) $F\left(\tau_{1}^{1}, \tau_{2}^{0}\right)$ and (b) $F\left(\tau_{1}^{0}, \tau_{2}^{0}\right)$ in the sandstone at $2 \mathrm{MHz}$. These 2D maps have the uniform color scale while their projected curves were normalized to better compare the pattern of the distributions.

As depicted in Fig. 4.10 (a), the eigenmodes correlation distribution $F\left(\tau_{1}^{1}, \tau_{2}^{0}\right)$ from $S\left(t_{\text {diff }}, N T_{\mathrm{E}}\right)$ is located below the diagonal line $\left(\tau_{1}=\tau_{2}\right)$. In contrast, the distribution of the ground mode correlation function $F\left(\tau_{1}^{0}, \tau_{2}^{0}\right)$ 
mainly aligns along the diagonal line and extends with a $\tau_{1} / \tau_{2}$ ratio of around 1.7, which implies a strong correlation of ground $T_{1}$ and $T_{2}$ modes at low-field. Both correlation maps in Fig. 4.10 have nearly identical $T_{2}$ distributions, indicating the common contribution from the ground $T_{2}$ mode in these two data sets. However, the $T_{1}$ values in $F\left(\tau_{1}^{1}, \tau_{2}^{0}\right)$ are smaller compared to the 1D projected $T_{1}$ of $F\left(\tau_{1}^{0}, \tau_{2}^{0}\right)$.

The $2 \mathrm{D}$ data sets $E\left(t_{\text {diff }}, N T_{\mathrm{E}}\right)$ and $R\left(t_{\text {diff }}, N T_{\mathrm{E}}\right)$ of the limestone were acquired and processed using the same procedure as for the sandstone. $I_{0,0}$ was calculated to be 0.75 according to the ratio of the first columns of $E\left(t_{\text {diff }}, N T_{\mathrm{E}}\right)$ and $R\left(t_{\text {diff }}, N T_{\mathrm{E}}\right)$ (Fig. $\left.4.11(\mathrm{a})\right)$, which again is close to $I_{0}(\approx$ $0.74)$ of the $1 \mathrm{D}$ result. Using the determined $I_{0,0}$, the $2 \mathrm{D}$ data $S\left(t_{\text {diff }}, N T_{\mathrm{E}}\right)$ was calculated from $E\left(t_{\text {diff }}, N T_{\mathrm{E}}\right)$ and $R\left(t_{\text {diff }}, N T_{\mathrm{E}}\right)$ with the result shown in Fig. 4.11 (b). As seen from this figure, the $2 \mathrm{D}$ data decay is slower along $t_{\text {diff }}$ axis compared to the data in Fig. 4.9 (b), which implies that larger decay time $\tau_{1}^{1}$ and bigger pores contribute to the signal in the limestone than the sandstone.

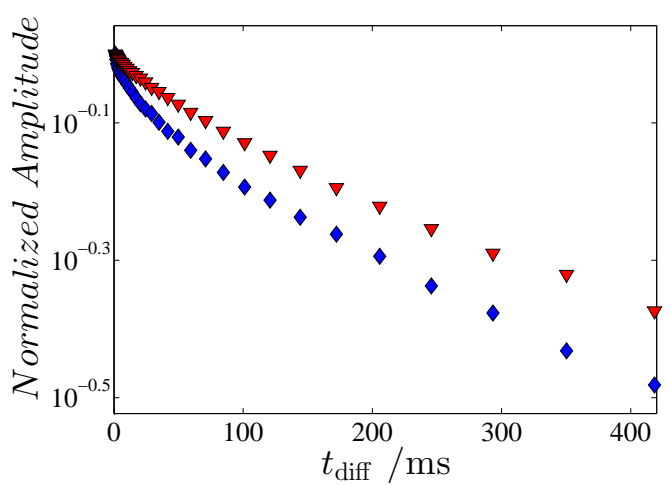

(a)

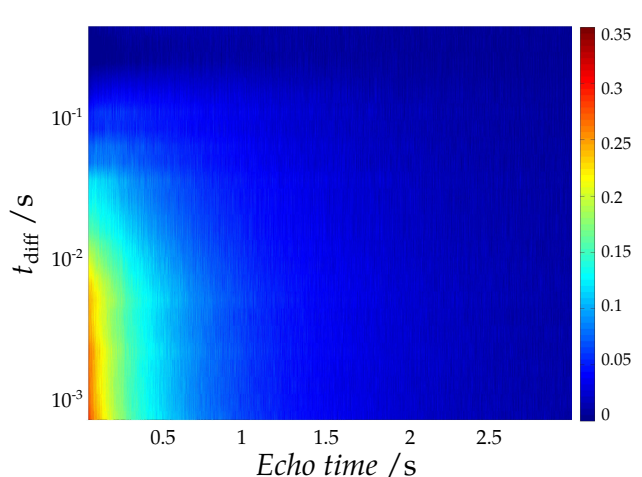

(b)

FIG. 4.11: (a) The first columns of the data matrices of $E\left(t_{\text {diff }}, N T_{\mathrm{E}}\right)$ (diamond) and $R\left(t_{\text {diff }}, N T_{\mathrm{E}}\right)$ (triangle) in the limestone; (b) the data set $S\left(t_{\text {diff }}, N T_{\mathrm{E}}\right)$ in limestone at $2 \mathrm{MHz}$. Only the data in the range of $t_{\text {diff }} 0.5 \mathrm{~ms}$ to $418 \mathrm{~ms}$ is shown.

Subsequently, the correlation function $F\left(\tau_{1}^{1}, \tau_{2}^{0}\right)$ was obtained from $S\left(t_{\text {diff }}, N T_{\mathrm{E}}\right)$ using 2D numerical inversion algorithm and was compared with the ground mode correlation function $F\left(\tau_{1}^{0}, \tau_{2}^{0}\right)$ from 2D reference data 
of limestone. The results of these two correlation functions are shown in Fig. 4.12. These two distributions are located in different zones of the $\tau_{1}-\tau_{2}$ correlation domain. Both 2D maps have almost identical $\tau_{2}$ but different $\tau_{1}$ distributions. The projected $1 \mathrm{D} \tau_{1}$ result in Fig. 4.12 (a) shifts towards smaller values compared with the $\tau_{1}$ distribution of $F\left(\tau_{1}^{0}, \tau_{2}^{0}\right)$ in Fig. 4.12 (b) similar to the case in the sandstone.

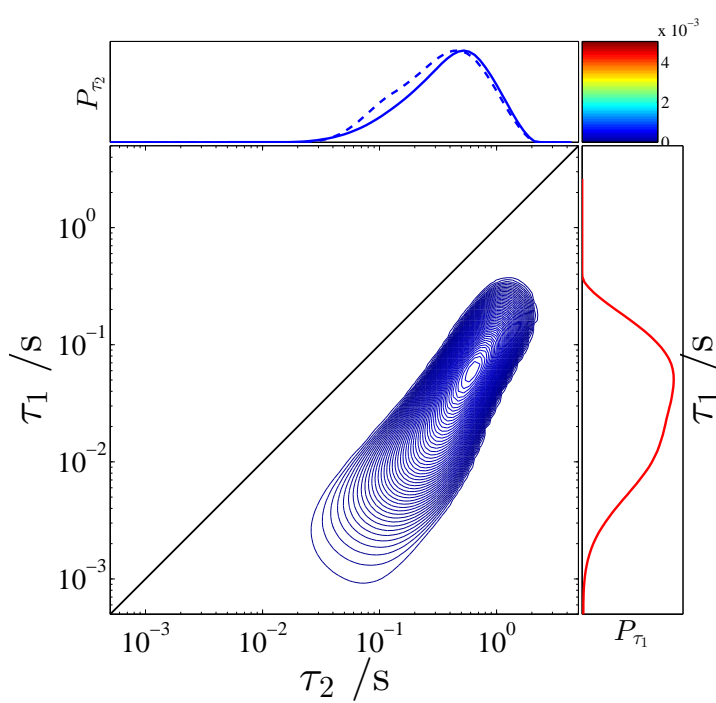

(a)

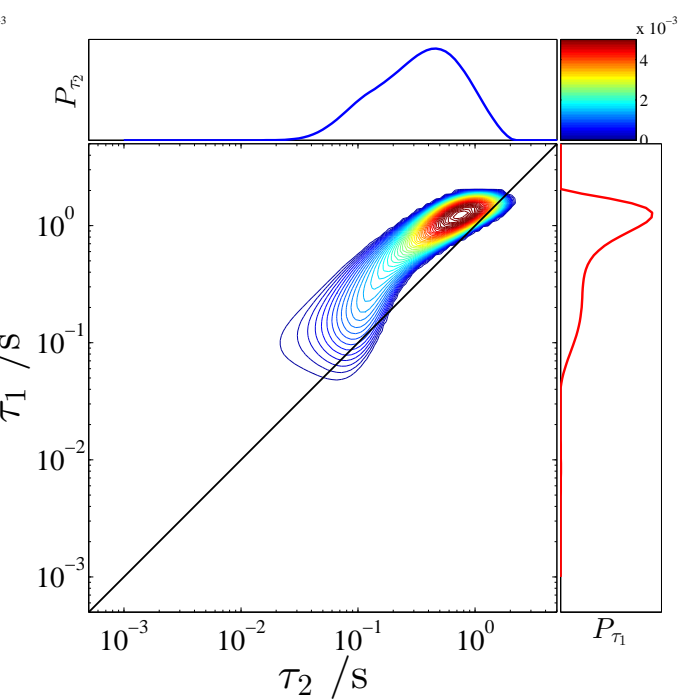

(b)

FIG. 4.12: Comparison of the distribution functions obtained from (a) $S\left(t_{\text {diff }}, N T_{\mathrm{E}}\right)$ and (b) $R\left(t_{\text {diff }}, N T_{\mathrm{E}}\right)$ in the limestone at $2 \mathrm{MHz}$. The uniform color scale was used in the $2 \mathrm{D}$ maps, but the projected $1 \mathrm{D}$ curves were normalized to better compare the distributions in $F\left(\tau_{1}^{1}, \tau_{2}^{0}\right)$ and $F\left(\tau_{1}^{0}, \tau_{2}^{0}\right)$.

To yield the correlation map of pore length $a$ and $T_{2}$, the acquired 2D correlation functions $F\left(\tau_{1}^{1}, \tau_{2}^{0}\right)$ were rescaled along the vertical axis via $a \approx 2 \zeta_{1} \sqrt{D \tau_{1}^{1}}$. The correlation functions $F\left(a, T_{2}\right)$ of the sandstone and limestone samples are shown in Fig. 4.13 along with the projections of pore length scales and $T_{2}$ relaxation distributions. In order to compare to the 1D results, data from Fig. 4.6 (b) and Fig. 4.7 (b) is presented using dashed lines.

As shown in Fig. 4.13 (a), the 1D projected pore length scales correspond well to the result from 1D experiment in sandstone, which confirms the result of $2 \mathrm{D}$ eigenmode correlation experiment. The $2 \mathrm{D}$ distribution can 


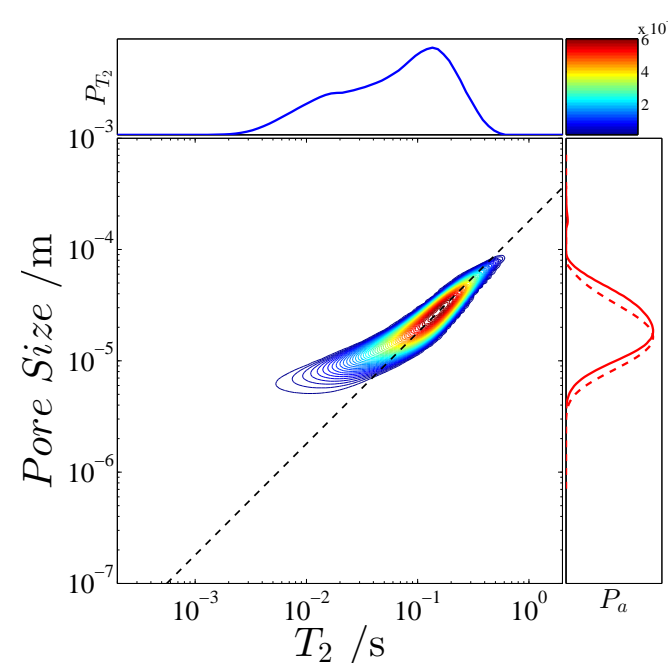

(a)

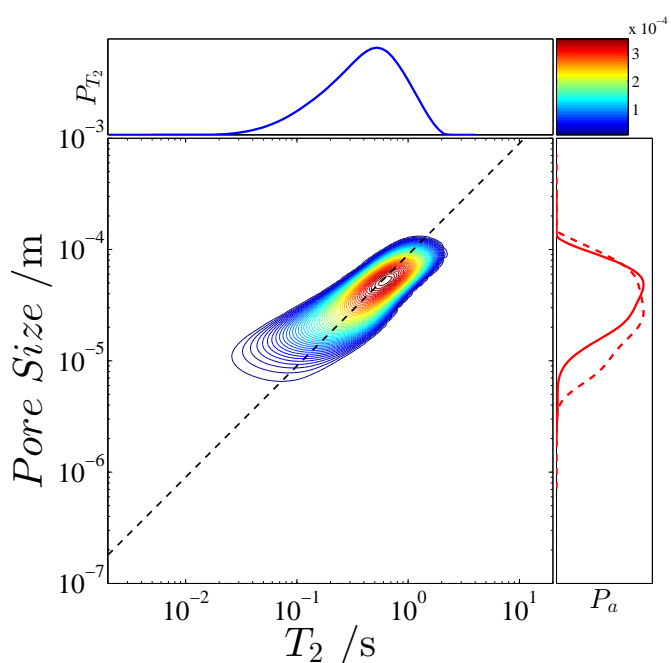

(b)

FIG. 4.13: The distributions of correlation functions $F\left(a, T_{2}\right)$ of sandstone (a) and limestone (b) at $2 \mathrm{MHz}$. The dashed lines in these 2D maps represent the correlation line with $\rho_{2}=30 \mu \mathrm{m} / \mathrm{s}$ in sandstone and $15 \mu \mathrm{m} / \mathrm{s}$ in limestone. The red dashed lines represent the pore length scales from 1D experiments and were normalized for the comparison with 1D projected pore size distribution from $F\left(a, T_{2}\right)$.

be separated into two parts according to the tendency of the distribution and each part exhibits different correlation information of pore length and $T_{2}$. For the distribution in the $T_{2}$ range from $0.1 \mathrm{~s}$ to $0.6 \mathrm{~s}$, it shows a strong linear relationship and lies along the dashed line representing the surface relaxivity of $\rho_{2}=30 \mu \mathrm{m} / \mathrm{s}$, which is a reasonable value in sandstone [168]. For the distribution in the $T_{2}$ range from $0.1 \mathrm{~s}$ to smaller values, the distribution deviates more from the dashed line with decreasing of $T_{2}$ value. The variation of the correlation map indicates the larger values of surface relaxivities occurred in the smaller pores, which may be caused by the existence of strong paramagnetic materials (clay for example) in those pore regions.

The correlation function $F\left(a, T_{2}\right)$ of the limestone sample lies along the correlated dashed line corresponding to the surface relaxivity of $\rho_{2}$ $=15 \mu \mathrm{m} / \mathrm{s}$, which demonstrates that the pore surface property of this rock is more homogeneous than that of the sandstone sample since it does not 
deviate from the dashed line. The estimated $\rho_{2}$ value is smaller compared to the result of sandstone, which is attributed to the small density of paramagnetic impurities at the pore surface. The $1 \mathrm{D}$ projected pore length scales corresponds reasonably well to the distribution from the 1D experiment although the result from the sandstone appears to be more consistent.

\section{Surface relaxivity $\rho_{2}$}

As discussed in Section 4.3.1, the surface relaxivity can be estimated from the 2D correlation function $F\left(a, T_{2}\right)$. The relationship of $T_{2}(a)$ can be established from calculating the logarithmic mean value of $T_{2}$ for each pore length $a$ in the 2D map of $F\left(a, T_{2}\right)$. As a result, the slope of $T_{2}(a)$ in the linear scale relates to the effective surface relaxivity of rock samples according to Eq. 4.26.

The results of $T_{2}(a)$ in sandstone and limestone were calculated from Fig. 4.13 and are illustrated in Fig. 4.14. Both results exhibit monotonic relationships of pore length and $T_{2}$ in the pore regions. Moreover, the sandstone curve lies above the limestone, which indicates larger surface relaxivities in sandstone than in limestone.

The slope of the result in sandstone varies in the different pore regimes, which implies heterogeneous surface properties in the pores. The surface relaxivity was estimated to be around $30 \mu \mathrm{m} / \mathrm{s}$ in the pore length ranging from $10 \mu \mathrm{m}$ to $70 \mu \mathrm{m}$, and was continuously increasing in the pores smaller than $10 \mu \mathrm{m}$, which was indicated in the inset of Fig. 4.14. These characteristics follow the distribution of $F\left(a, T_{2}\right)$ in Fig. 4.13 (a) and confirm the larger surface relaxivities $\rho_{2}$ in smaller pores. In the result of limestone, the plot is mostly linear for $T_{2}$ values up to $1 \mathrm{~s}$. The surface relaxivity in limestone was then estimated to be $15 \mu \mathrm{m} / \mathrm{s}$, which matches well with the result of the dashed line in Fig. 4.13 (b). The uprising of the tail above $1 \mathrm{~s}$ was caused by the particular shape of the 2D map which may lead to a slightly distorted functional dependency of $T_{2}(a)$ when using the log mean of $T_{2}$ along the values of $a$. A different averaging method more suitable 
for the shape of the contour may lead to more consistent result but were not explored in the context of this work. Besides that, the complexity of the pore shape will break the assumption of spherical pores in Eq. 4.26 and might introduce the deviation of the result.

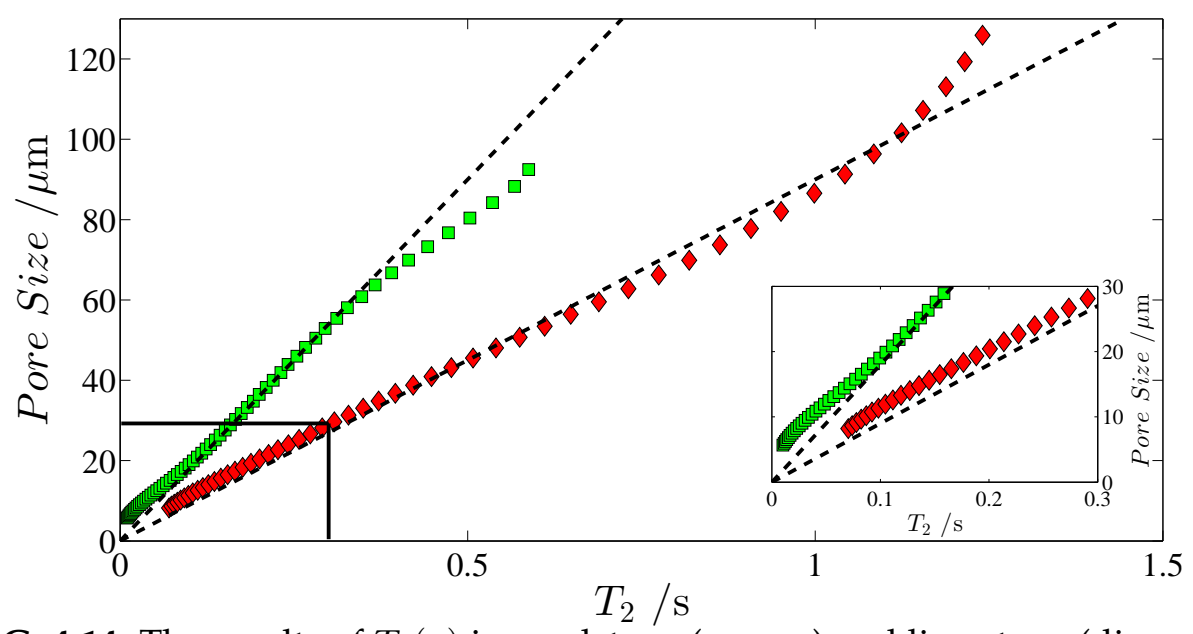

FIG. 4.14: The results of $T_{2}(a)$ in sandstone (square) and limestone (diamond). To better present the feature of surface relaxivities in these two rock samples, the transposed data is plotted. The straight dashed lines corresponding to the surface relaxivities of $30 \mu \mathrm{m} / \mathrm{s}$ and $15 \mu \mathrm{m} / \mathrm{s}$ are plotted to show the linearity of pore length $a$ and $T_{2}$. A geometrical factor of 6 is considered according to Eq. 4.26. The inset shows the $T_{2}(a)$ results in smaller pore regime.

In order to validate the obtained surface relaxivities, two critical characteristic lengths for molecular diffusion in pore space are compared in these two rock cores. An effective length $l_{\rho}=D / \rho_{2}$ is used in order to characterize the distance travelled by the molecules between the first collision onto the surface sink [231]. This characteristic length is estimated to be $80 \mu \mathrm{m}$ in this sandstone and $160 \mu \mathrm{m}$ in the limestone. Since there are two dimensions of measurements, pore length $a$ and $T_{2}$, affecting the determination of surface relaxivities, the diffusion length $l_{d}$ along these two domains are evaluated, respectively. For diffusion length along $T_{2}$ measurement domain, $l_{d}=\sqrt{D T_{\mathrm{E}}}=0.55 \mu \mathrm{m}$ for $T_{\mathrm{E}}=125 \mu \mathrm{s}$. For diffusion length along pore scale domain, the largest pore size is $70 \mu \mathrm{m}$ in sandstone and $130 \mu \mathrm{m}$ in limestone, which corresponds to the longest diffusion observation time 
$t_{\text {diff }}$ of $100 \mathrm{~ms}$ and $400 \mathrm{~ms}$, therefore, the diffusion lengths under these times are $16 \mu \mathrm{m}$ and $32 \mu \mathrm{m}$. Both cases indicate that the diffusion length in $2 \mathrm{D}$ experiment is smaller than the characteristic length $l_{\rho}$, which implies that the molecules in the pore experience enough collision close to the surface. As a result, the pore surface area should be well sampled by the molecular mobility during the $2 \mathrm{D}$ experiment and the obtained surface relaxivites should be consistent.

To validate this conclusion, the surface relaxivities of these two rock samples were measured using Padé approximation from the 2D $D-T_{2}$ correlation map [228]. The formation factor $F$ was independently measured to be 40.8 in sandstone and 7.5 in limestone. The diffusion encoding time $\Delta$ in 2D $D-T_{2}$ correlation experiment was $45 \mathrm{~ms}$. These parameters were then put into the Padé approximation procedure as the necessary factors in Eq. 4.15. The 2D experimental results are given in Fig. 4.15 and the estimated surface relaxivities $\rho_{2}$ were $25 \mu \mathrm{m} / \mathrm{s}$ in sandstone and $12 \mu \mathrm{m} / \mathrm{s}$ in limestone. The values correspond well with the results of $\rho_{2}$ in the limestone and was underestimated in the sandstone. This inconsistency is probably due to the non-negligible internal gradient effect during the diffusion encoding time $\Delta$.

It is worth noting that the experimental data acquired from the Padé approximation method will be additionally attenuated because of the relaxation effect presence during the diffusion encoding time $\Delta$. This effect is relatively weak in the eigenmode correlation experiments because of the shorter phase encoding period $t_{\mathrm{e}}$ compared to $\Delta$ in Padé diffusionrelaxation correlation. As a result, the surface information of the pores with comparably small size and significant internal gradients can be preserved and evaluated using the eigenmodes correlation method. 


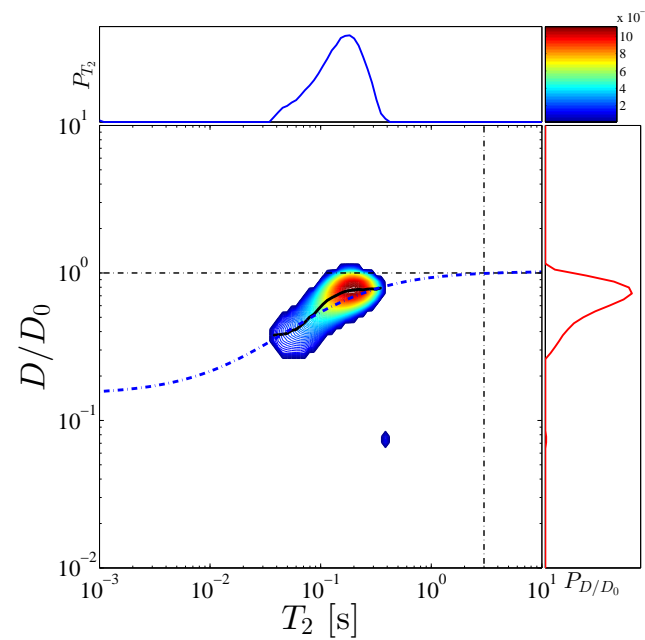

(a)

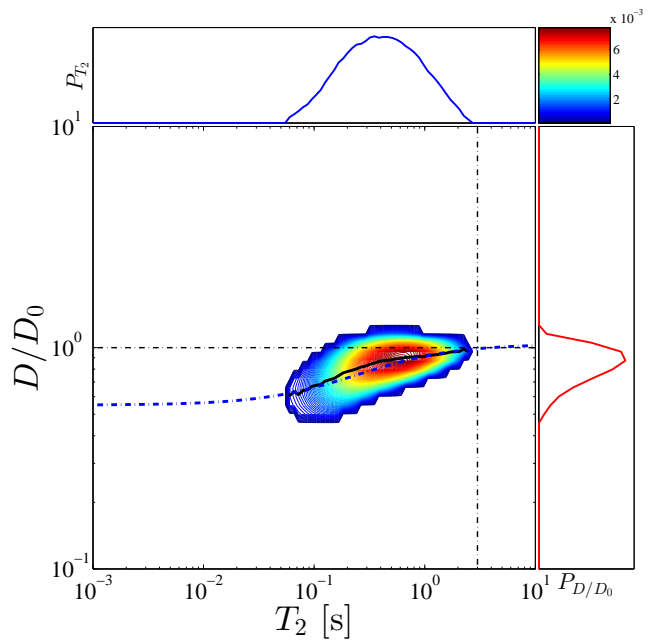

(b)

FIG. 4.15: Padé approximation on the correlation functions $F\left(D, T_{2}\right)$ in sandstone (a) and limestone (b) at $2 \mathrm{MHz}$. The horizontal and vertical dashed lines in these 2D maps represent the normalized diffusion coefficient line and the bulk $T_{2}$ relaxation time for water, respectively. The black solid lines are the correlation of logarithmic mean value of $D$ distribution along $T_{2}$ domain and the blue dashed lines are the Padé approximation of the black solid lines using Eq. 4.15. The fitting results yield that $\rho_{2}=25 \mu \mathrm{m} / \mathrm{s}$ in sandstone and $12 \mu \mathrm{m} / \mathrm{s}$ in limestone.

\subsection{3 $T_{1}-T_{1}$ eigenmode correlation results}

\section{D correlation results}

The acquired 2D data of the rock samples by using the pulse sequence given in Fig. 4.4 were processed using 2D ILT and the results were shown in Fig. 4.16 (a) for Nugget sandstone and Fig. 4.17 (a) for Winterset limestone. Meanwhile, independent experiments were performed based on the pulse sequence given in Fig. 4.5, only for validating the experimental results of first and ground $T_{1}$ mode correlation. The results from this experiment were given in Fig. 4.16 (b) for Nugget sandstone and Fig. 4.17 (b) for Winterset limestone.

From the comparison of the experimental results from Nugget sandstone and Winterset limestone shown in Fig. 4.16 (b) and Fig. 4.17 (b), it is easy to observe that the ground $T_{1}$ self-correlation distribution mainly 


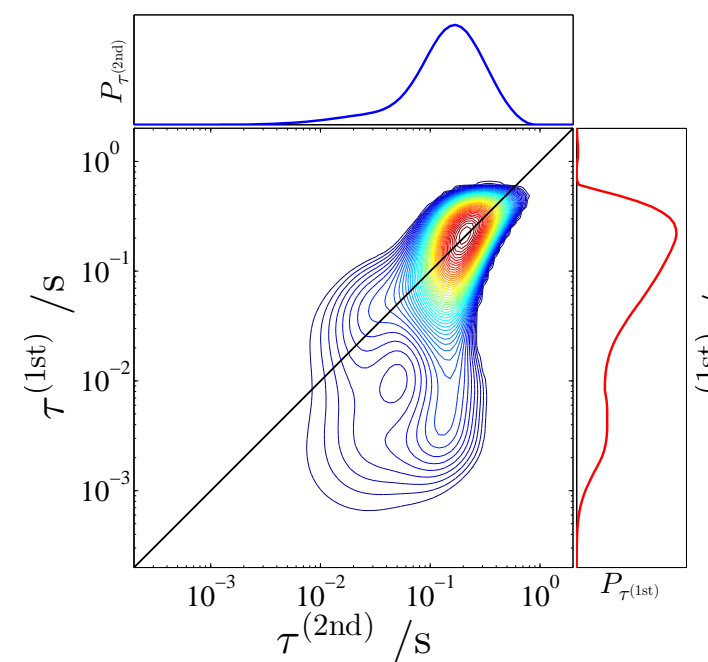

(a)

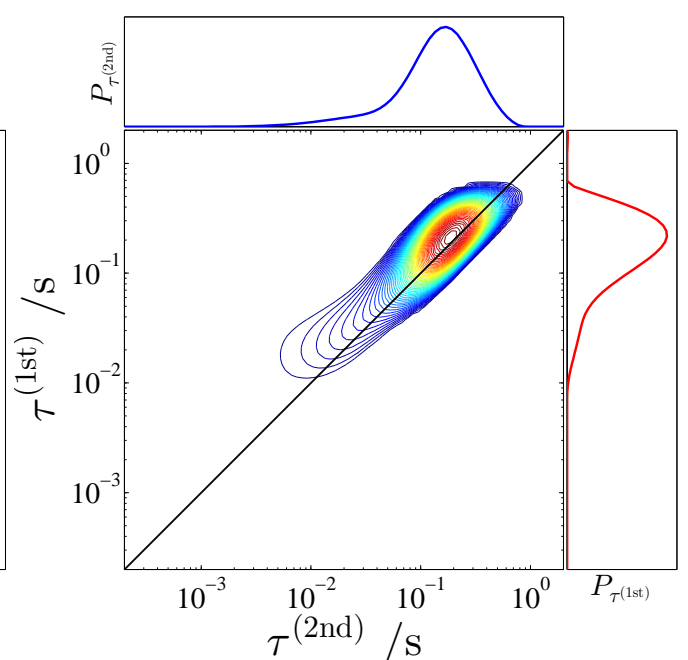

(b)

FIG. 4.16: The ground and first $T_{1}$ mode correlation map (a) and ground $T_{1}$ mode self-correlation map (b) in Nugget sandstone obtained according to Eq. 4.21. The solid diagonal lines represent equal eigenvalues in 2D correlation maps.

lies along the diagonal line, which proves that only the ground $T_{1}$ mode contributed during the $1^{\text {st }}$ and $2^{\text {nd }}$ domain of this $2 \mathrm{D}$ experiment. However, in the ground and first $T_{1}$ eigenmode correlation results shown in Fig. 4.16 (a) and Fig. 4.17 (a), there exist two discernible areas in the distribution, one along the diagonal line and a second one below the diagonal line. The part aligning along the diagonal line stands for the signal from the ground $T_{1}$ mode correlation while the part below the diagonal line represents the signal from the ground and first $T_{1}$ mode correlation. Since the first $T_{1}$ eigenvalue is smaller than the ground $T_{1}$ eigenvalue, this part of the distribution is located below the diagonal line. These two parts, if explained by the NMR response equation, exactly correspond to the two exponential terms in Eq. 4.21. Moreover, by comparing the 1D projected curves in the top and right panels of 2D correlation maps, we can observe that there are comparable $\tau_{1}$ values in the major section of these two 1D distributions, and extra segment with smaller $\tau_{1}$ values in the right panel. 


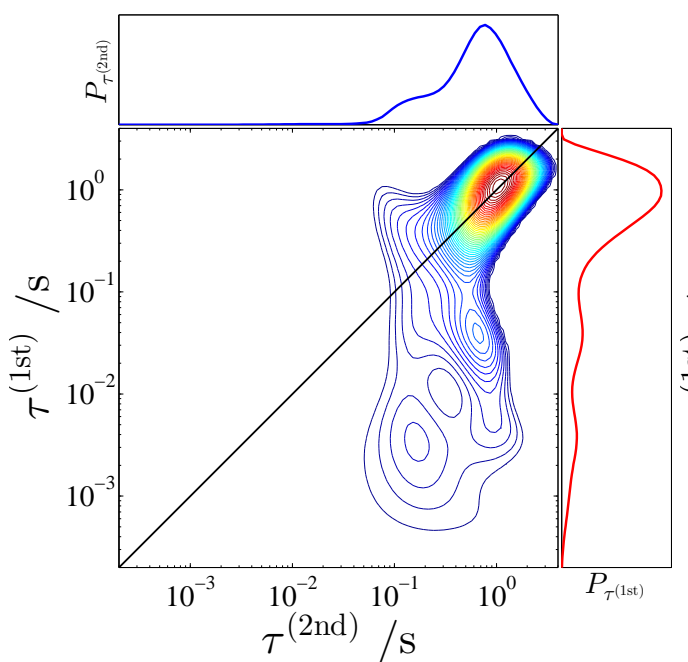

(a)

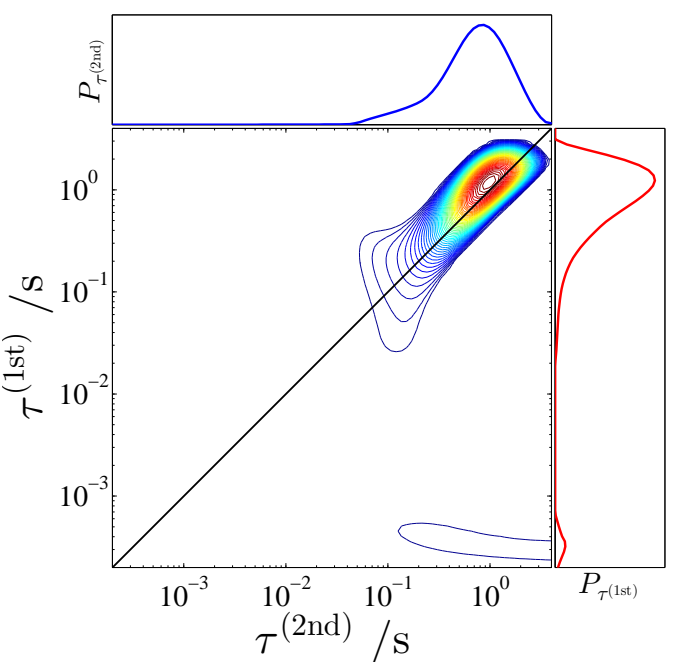

(b)

FIG. 4.17: The ground and first $T_{1}$ mode correlation map (a) and ground $T_{1}$ mode self-correlation map (b) in Winterset limestone obtained according to Eq. 4.21. The solid diagonal lines represent equal eigenvalues in 2D correlation maps.

This is a straightforward evidence that there is only ground $T_{1}$ eigenmode contribution in the $2^{\text {nd }}$ domain of the acquisition, while in the $1^{\text {st }}$ domain, the ground and first $T_{1}$ mode are both detected. In order to extract the pore size information, the contribution from the ground $T_{1}$ mode in the $1^{\text {st }}$ domain needs to be eliminated.

As already mentioned in Section 4.2.2, the isolation of the ground $T_{1}$ mode contribution in the $1^{\text {st }}$ domain relies on the data from $2^{\text {nd }}$ domain rather than an extra reference acquisition. In order to illustrate the steps to obtain the ground and first $T_{1}$ correlation map, we briefly revisit these steps here:

1: Calibrating the amplitudes of $2 \mathrm{D}$ signal $E\left(t_{\text {diff }},(N-1) t_{\mathrm{a}}\right)$ in Eq. 4.21.

2: Obtaining the correlation distribution $F\left(\tau^{(1 s t)}, \tau^{(2 n d)}\right)$ from the calibrated $E\left(t_{\text {diff }},(N-1) t_{\mathrm{a}}\right)$ using 2D ILT. As indicated in Fig. 4.16 (a) and Fig. 4.17 (a), the obtained distribution function is $F\left(\left[\tau_{0}, \tau_{1}\right], \tau_{0}\right)$. 
3: Evaluating the relative intensities of the ground $T_{1}$ mode $I_{0,0}$ and then eliminate it from the signal in the $1^{\text {st }}$ domain.

4: Obtaining the data matrix $S\left(t_{\text {diff }},(N-1) t_{\mathrm{a}}\right)$ given in Eq. 4.22. Processing it using $2 \mathrm{D}$ ILT to be $F\left(\tau_{1}, \tau_{0}\right)$ and rescale it further to $F\left(a, T_{1}\right)$.

Therefore, it is notable that the determination of factor $I_{0,0}$ is critical in the calculation of $F\left(\tau_{1}, \tau_{0}\right)$. As discussed in Section 4.2, $I_{0,0}=\int \varphi_{1}^{0} d V$. $\int \varphi_{1}^{0} \varphi_{1}^{0} d V \cdot \int \sin \Phi \varphi_{1}^{0} d V \approx \int \varphi_{1}^{0} d V \cdot \int \sin \Phi \varphi_{1}^{0} d V$, which is approximately the value of $I_{0}$ calculated in $1 \mathrm{D}$ experiment. As a result, $I_{0}$, instead of $I_{0,0}$, will be estimated in this case.

In order to obtain this factor, the first row (green circle) and first column (blue diamond) of the 2D data matrices after Step 1 were extracted and plotted in Fig. 4.18 (a) and Fig. 4.19 (a). The first row stands for the signal attenuation due to the ground $T_{1}$ mode only and the first column for the decay dominantly caused by the ground and first $T_{1}$ modes. That is the reason why the dataset along the $1^{\text {st }}$ domain decay faster than the data along the $2^{\text {nd }}$ domain of the acquisition. By fitting the later dataset using $1 D$ ILT, a new data vector representing the ground $T_{1}$ mode contribution under each $t_{\text {diff }}$ can then be interpolated and shown as red triangle. Consequently, the value of $I_{0}$ can be determined using the ratio of the data along the $1^{\text {st }}$ domain and the rebuilt data vector at long $t_{\text {diff. }}$.

After consecutively applying this obtained value in the 2D data matrices, Step 4 can be carried out to obtain the data matrix $S\left(t_{\text {diff }},(N-1) t_{\mathrm{a}}\right)$. The 2D datasets in these two rock cores are shown in Fig. 4.18 (b) and Fig. 4.19 (b). The data attenuate more rapidly along the $t_{\text {diff }}$ domain in these two surf maps, revealing the high $T_{1}$ mode contribution in this dimension. Then the ground and first $T_{1}$ mode correlation function $F\left(\tau_{1}, \tau_{0}\right)$ can be obtained and shown in Fig. 4.20. By comparing and observing the location and range of these two distributions, we notice that they exhibit shorter $T_{1}$ eigenvalue in the $1^{\text {st }}$ domain which correspond to the distributions below the diagonal lines in Fig. 4.16 (a) and Fig. 4.17 (a), respectively. 


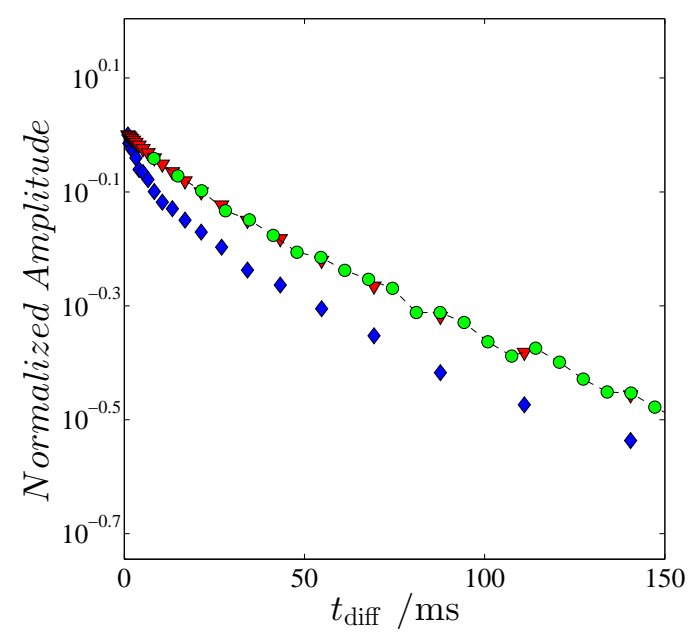

(a)

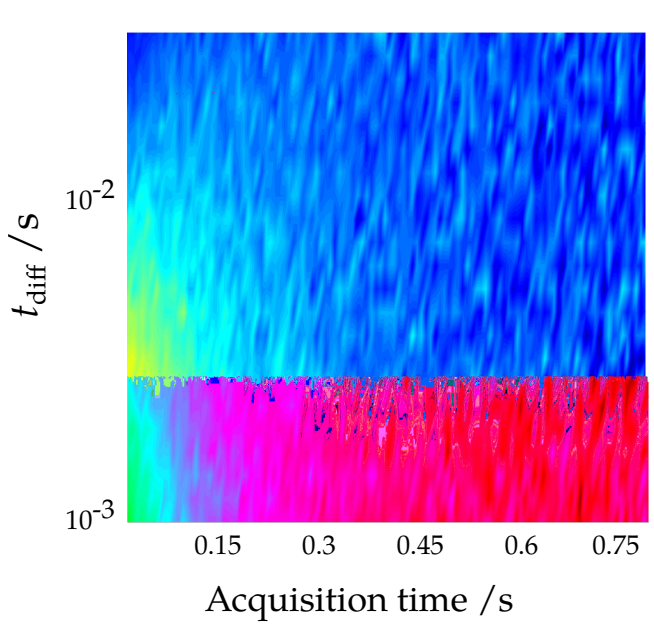

(b)

FIG. 4.18: (a) The data sets from $1^{\text {st }}$ domain (diamond) and from $2^{\text {nd }}$ domain (circle). The triangle markers represent the reconstructed data set corresponding to the same $t_{\text {diff }}$ of data acquired in the first domain; (b) the $S\left(t_{\text {diff }},(N-1) t_{\mathrm{a}}\right)$ data matrix in sandstone at $64 \mathrm{MHz}$.

The obtained correlation function $F\left(\tau_{1}, \tau_{0}\right)$ can be further translated to $F\left(a, T_{1}\right)$ along the vertical axis via $a \approx 2 \zeta_{1} \sqrt{D \tau_{1}^{1}}$. The correlation functions $F\left(a, T_{1}\right)$ of the sandstone and limestone samples are given in Fig. 4.21 along with the projections of pore length scales and $T_{2}$ relaxation distributions. In order to compare to the 1D results, data from Fig. 4.8 (b) and Fig. 4.7 (b) is presented using red dashed lines.

In both cases, the 1D projected pore length scales correspond well to the $1 \mathrm{D}$ experimental results, which confirms the result of $2 \mathrm{D} T_{1}-T_{1}$ eigenmode correlation experiment. The $2 \mathrm{D}$ distribution functions $F\left(a, T_{1}\right)$ are broadened as compared to the obtained $F\left(a, T_{2}\right)$ from $T_{1}-T_{2}$ eigenmode correlation experiment given in Fig. 4.13, which is due to the bigger smoothing parameters used in the ILT because of the data amplitudes calibration in the Step 1 of the data processing. Nevertheless, strong correlation of pore length and $T_{1}$ relaxation can still be observed from these $2 \mathrm{D}$ maps. 


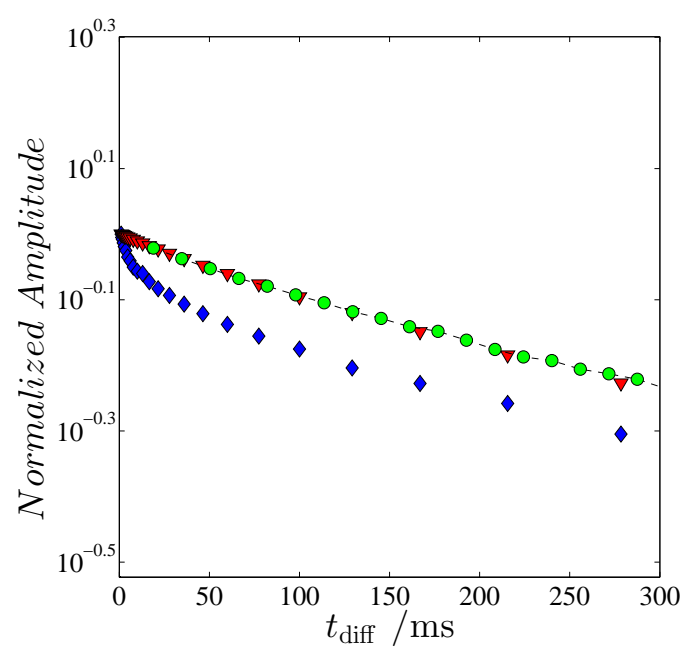

(a)

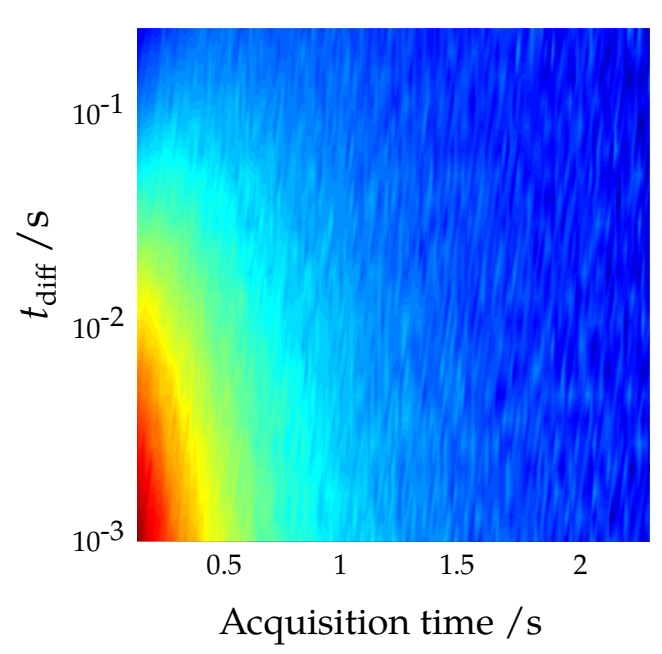

(b)

FIG. 4.19: (a) The data sets from $1^{\text {st }}$ domain (diamond) and from $2^{\text {nd }}$ domain (circle). The triangle markers represent the reconstructed data set corresponding to the same $t_{\text {diff }}$ of data acquired in the first domain; (b) the $S\left(t_{\text {diff }},(N-1) t_{\mathrm{a}}\right)$ data matrix in limestone at $64 \mathrm{MHz}$.

The dashed lines representing the surface relaxivity of $\rho_{1}=18 \mu \mathrm{m} / \mathrm{s}$ in the sandstone and $\rho_{1}=8 \mu \mathrm{m} / \mathrm{s}$ in the limestone can be marked to indicate the tendency and correlation of the obtained $F\left(a, T_{1}\right)$ maps.

\section{Surface relaxivity $\rho_{1}$}

As discussed in Section 4.3.2, the surface relaxivity can be estimated from the $2 \mathrm{D}$ correlation function $F\left(a, T_{1}\right)$. The relationship of $T_{1}(a)$ can be established from calculating the logarithmic mean value of $T_{1}$ for each pore length $a$ in the 2D map of $F\left(a, T_{1}\right)$. As a result, the slope of $T_{1}(a)$ in the linear scale relates to the effective surface relaxivity of rock samples according to Eq. 4.27.

The results of $T_{1}(a)$ in sandstone and limestone were calculated from Fig. 4.21 and plotted in Fig. 4.22. Both results exhibit monotonic relationships of pore length and $T_{1}$ in the pore regions. Moreover, the sandstone 


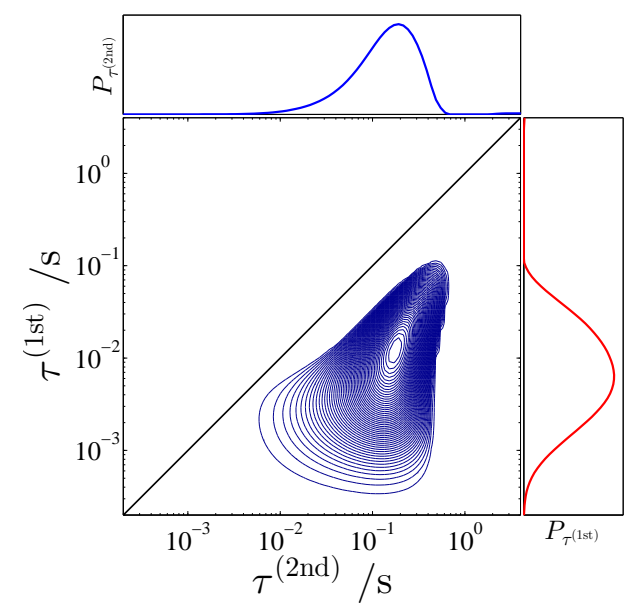

(a)

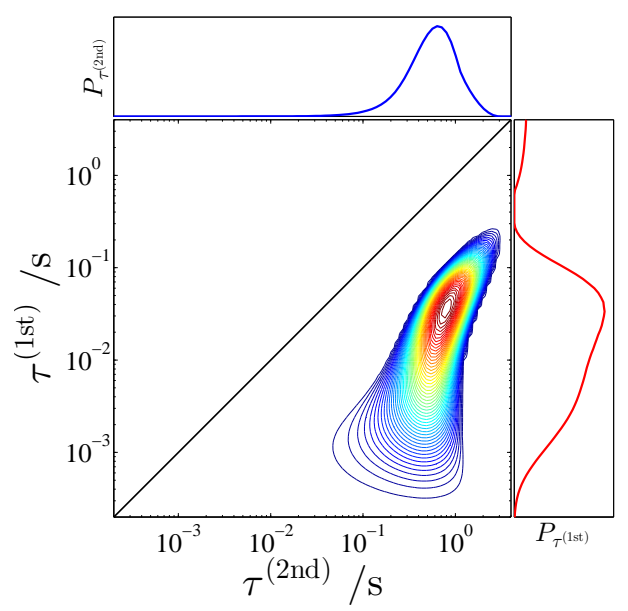

(b)

FIG. 4.20: The first and ground $T_{1}$ mode correlation map in Nugget sandstone (a) and Winterset limestone (b) obtained according to Eq. 4.22.

curve lies above the limestone, which indicates larger surface relaxivities in sandstone than in limestone. The surface relaxivity of sandstone was estimated to be around $18 \mu \mathrm{m} / \mathrm{s}$ in the pore length ranging from $10 \mu \mathrm{m}$ to $70 \mu \mathrm{m}$, and was continuously increasing in the pores smaller than 10 $\mu \mathrm{m}$. These characteristics follow the distribution of $F\left(a, T_{1}\right)$ in Fig. 4.21 (a) and confirm the larger surface relaxivities $\rho_{1}$ in smaller pores. The surface relaxivity in Winterset limestone was then estimated to be $8 \mu \mathrm{m} / \mathrm{s}$, which matches the result of the dashed line in Fig. 4.21 (b). Similar to the case of surface relaxivity $\rho_{2}$, the complexity of the actual pore shape as compared to the assumed spherical pore in Eq. 4.27 may introduce further deviations from a simple linear relationship. 


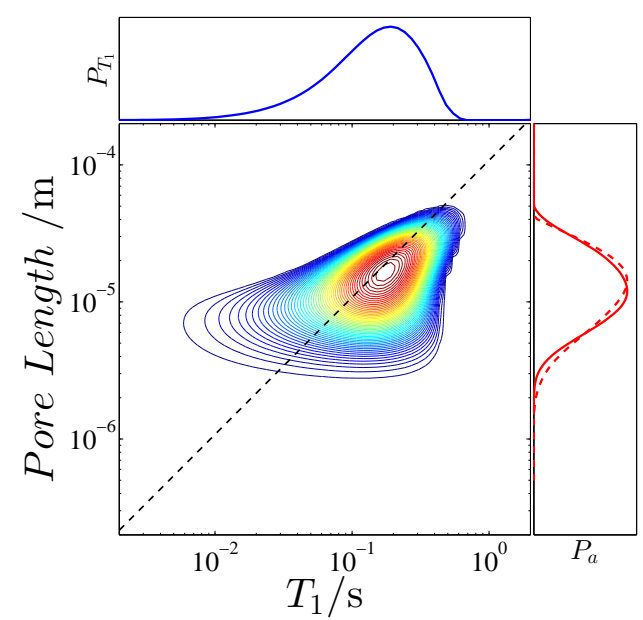

(a)

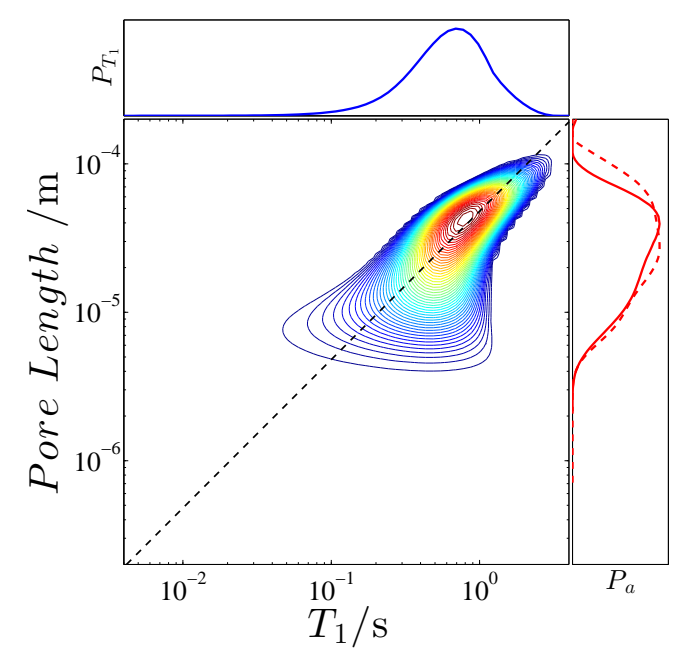

(b)

FIG. 4.21: The distributions of correlation functions $F\left(a, T_{1}\right)$ of Nugget sandstone (a) and Winterset limestone (b). The dashed lines in these 2D maps represent the correlation line with $\rho_{1}=18 \mu \mathrm{m} / \mathrm{s}$ in sandstone and $8 \mu \mathrm{m} / \mathrm{s}$ in limestone. The red dashed lines represent the pore length scales from 1D experiments and were normalized for the comparison with 1D projected pore size distribution from $F\left(a, T_{1}\right)$.

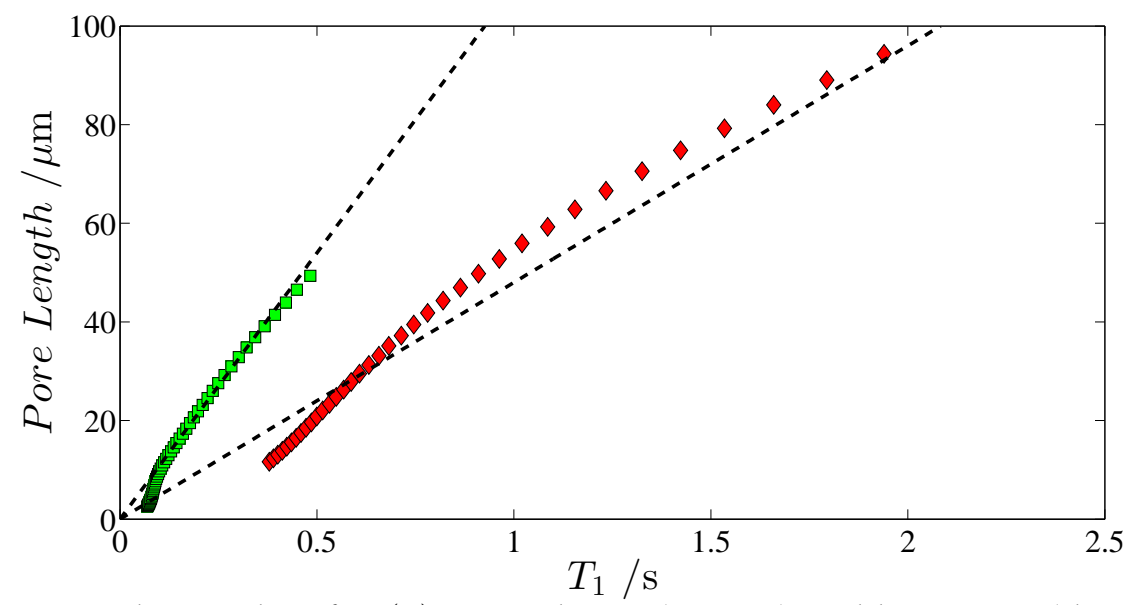

FIG. 4.22: The results of $T_{1}(a)$ in sandstone(square) and limestone (diamond). To better present the feature of surface relaxivities in these two rock samples, the transposed data is plotted. The straight dashed lines corresponding to the surface relaxivities of $18 \mu \mathrm{m} / \mathrm{s}$ and $8 \mu \mathrm{m} / \mathrm{s}$ are plotted to show the linearity of pore length $a$ and $T_{1}$. A geometrical factor of 6 is considered according to Eq. 4.27. 


\section{Chapter 5}

\section{Local Permeability Estimation}

In order to estimate permeability from relaxation time distribution, relaxation property of rock cores were spatially resolved along the cylindrical axis using magnetic resonance imaging. Moreover, a factor defining the local connectedness can be extracted from calculating the correlation degree of adjacent relaxation time distributions from the neighbouring two resolved slices. Consequently, a permeability profile can be estimated and the local connectivity property of rock cores can be characterized considering the local porosity $\phi$, relaxation time distribution, and the connected factor consecutively along cylindrical axis.

\subsection{Methodology}

As discussed in Chapter 3, surface relaxation $T_{1}$ and $T_{2}$ distributions of rock samples indicate pore length information and can be used to estimate permeability. In this chapter, we choose $T_{1}$ relaxation since it is intrinsically immune to the induced internal magnetic fields $B_{i n}$. Before going deep into the heart of this chapter, we firstly review a unique method suggested in [32], which yields $T_{1}$ relaxation distribution in a rapid way. 


\subsubsection{Fast encoding $T_{1}$ relaxation}

The inversion recovery pulse sequence shown in Fig. 2.8 is traditionally utilized for $T_{1}$ measurement. However, it is a time-consuming technique since the spin system needs to recover to thermal equilibrium state before recording the magnetization under each evolution time $T_{\mathrm{W}}$. There are many techniques that have been developed to accelerate the $T_{1}$ acquisition $[32,232-234]$. One of these approaches, using small tip angle pulses to encode $T_{1}$ information [32], is shown in Fig. 5.1. This approach, which has been used in Chapter 4 for encoding the ground relaxation eigenmode, will be employed as the base of the imaging pulse sequence in this chapter.

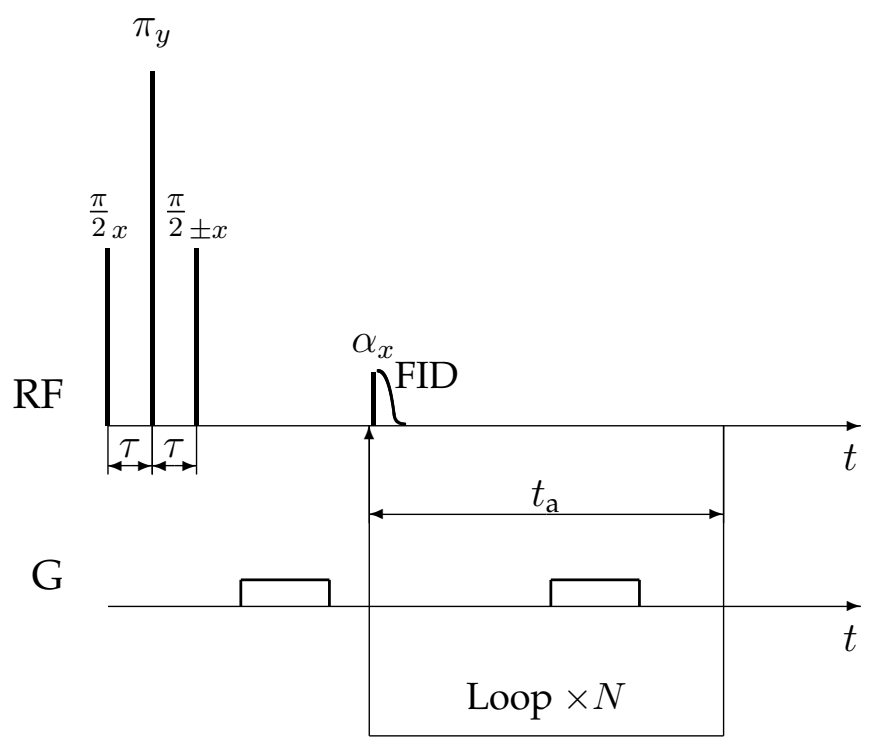

FIG. 5.1: Pulse sequence for rapidly encoding $T_{1}$ information [32]. $\alpha$ represents the tip angle of the looping pulses and the blocks along $G$ track are homo-spoil gradients for cancelling the unwanted magnetization existing in the transverse plane. $t_{\mathrm{a}}$ is the encoding spacing of two succesive $\alpha$ pulses. A two-step phase cycling is used for the second $\pi / 2$ pulse to select the decay pattern of the magnetization evolution along the longitudinal axis.

The idea of this pulse sequence is recording the magnetization decay aligning along the longitudinal axis rather than its recovery procedure. 
With the first three pulses tipping the magnetization to the longitudinal axis, a train of small tip angle pulses is applied later on to manipulate this magnetization. By using a two-step phase cycling of the second $\pi / 2 \mathrm{RF}$ pulse [32], the magnetization after each small $\alpha$ pulse can be expressed as:

$$
M\left((N-1) \cdot t_{\mathrm{a}}\right)=2 M_{0} \sin \alpha \cdot \exp \left(-\frac{(N-1) \cdot t_{\mathrm{a}}}{T_{1}}\right) \cdot \cos ^{N-1} \alpha
$$

where $N$ represents the number of the small tip angle pulses and $t_{\mathrm{a}}$ is the signal evolution time between the adjacent FID acquisition. This magnetization attenuation is actually identical to Eq. 4.19 in Chapter 4, which expresses the signal decay due to the ground $T_{1}$ mode.

The signal evolution of this technique is a "CPMG-like" attenuation and therefore this measurement speeds up the $T_{1}$ acquisition without the needs of recovering the magnetization back to thermal equilibrium. For the multiple $T_{1}$ components which exist in porous materials, Eq. 5.1 can be extended to as:

$M\left((N-1) \cdot t_{\mathrm{a}}\right)=2 M_{0} \int F\left(T_{1}\right) \cdot\left[\exp \left(-\frac{(N-1) \cdot t_{\mathrm{a}}}{T_{1}}\right) \sin \alpha \cdot \cos ^{N-1} \alpha\right] d T_{1}$.

Before obtaining the $T_{1}$ distribution function $F\left(T_{1}\right)$ by using ILT, the measured signal needs to be calibrated via dividing the intensities of FID signals by corresponding factors $\cos ^{N-1} \alpha$. For the practical purpose, a compromising loop number $N$ can be chosen in the range of $[50,200]$ considering the number of homo-spoil gradients used and the data points we need to record according to $T_{1}$ relaxation. Moreover, it is worth noting that the essential of this fast technique is the utility of small tip angle pulses. This advantage, however, will deliver a comparably low SNR for the acquired signal. Therefore, it is critical to choose an optimized tip angle $\alpha$, especially in the low-field application. Firstly, $\alpha$ can not be too small since the SNR of the acquired data is proportional to $\sin \alpha$. Meanwhile, $\alpha$ should not be too large, otherwise only a limited number of data points can be 
acquired. A good starting point to optimize $\alpha$ in this experiment would be the Ernest angle, which is estimated by $\arccos \left[\exp \left(-t_{\mathrm{a}} / T_{1}\right)\right]$. Therefore, an acceptable $\alpha$ in the range of $5^{\circ}$ to $9^{\circ}$ is advisable from our experimental experience.

\subsubsection{Spatially-resolved $T_{1}$ relaxation profile}

After introducing this fast $T_{1}$ encoded method, we extend it to resolve the $T_{1}$ information along a spatial axis by combing it with an imaging technique, in order to obtain the local relaxation information of the measured samples. The pulse sequence for spatially resolved $T_{1}$ mapping is shown in Fig. 5.2 and the pulse phase cycling given in Tab. 5.1.

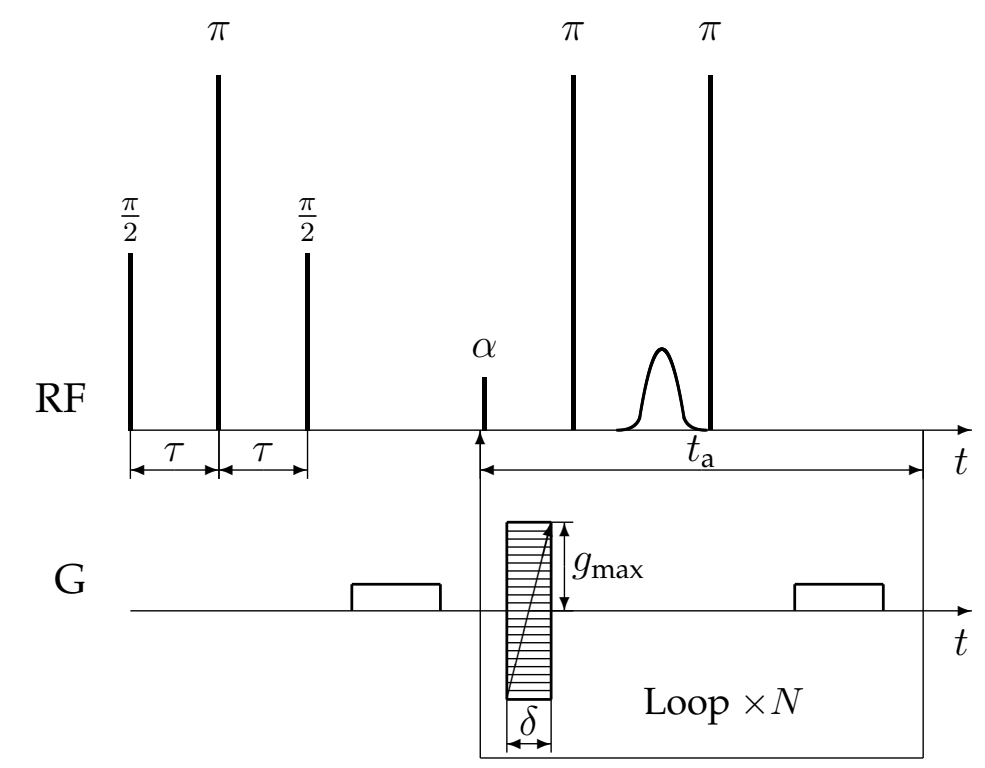

FIG. 5.2: Pulse sequence for spatially resolved $T_{1}$ mapping. The number of the acquired echoes is $N$ and $\delta$ is the phase encoded gradient. This gradient ramps from $-g_{\max }$ up to $g_{\max }$ linearly in $m$ steps.

This pulse programme has two major modifications based on the pulse sequence shown in Fig. 5.1. Firstly, a phase gradient is applied after the $\alpha$ pulse to encode the spatial information. The $k$-space pattern is then 
Table 5.1: Phase cycling used in phase encoded $T_{1}$ imaging in Fig. 5.2. $\phi_{1}, \phi_{2}, \phi_{3}$, $\phi_{4}$ and $\phi_{A c q}$, stand for the phases of the first $\pi / 2$ pulse, the first $\pi$ pulse, the second $\pi / 2$ pulse, the following $\pi$ pulses and the acquisition phase.

\begin{tabular}{ccccc}
\hline$\phi_{1}$ & $\phi_{2}$ & $\phi_{3}$ & $\phi_{4}$ & $\phi_{A C Q}$ \\
\hline$\pi / 2$ & $\pi$ & $\pi / 2$ & $\pi$ & 0 \\
$\pi / 2$ & $\pi$ & $3 \pi / 2$ & $\pi$ & $\pi$ \\
$3 \pi / 2$ & $\pi$ & $\pi / 2$ & $\pi$ & 0 \\
$3 \pi / 2$ & $\pi$ & $3 \pi / 2$ & $\pi$ & $\pi$ \\
$\pi / 2$ & $\pi$ & $\pi / 2$ & $\pi / 2$ & 0 \\
$\pi / 2$ & $\pi$ & $3 \pi / 2$ & $\pi / 2$ & $\pi$ \\
$3 \pi / 2$ & $\pi$ & $\pi / 2$ & $\pi / 2$ & 0 \\
$3 \pi / 2$ & $\pi$ & $3 \pi / 2$ & $\pi / 2$ & $\pi$ \\
\hline
\end{tabular}

recorded in the peak amplitude of the following echo. Secondly, an extra $\pi$ pulse needs to be applied after the echo acquisition. This pulse is necessary because of the additional $\pi$ pulse just after the $\alpha$ pulse. While the additional $\pi$ pulse after the $\alpha$ pulse refocuses the magnetization in the transverse plane (and form an echo) it simultaneously rotates the $z$-component of the magnetization as described in Eq. 4.18 to the opposite direction. This will distort the magnetization evolution along the $z$ direction and the $T_{1}$ acquisition using small tip angle according to Eq. 5.1 fails. Therefore, the rotated $z$-component magnetization needs to be tipped back to its original direction using the extra $\pi$ pulse after the echo.

By using this pulse sequence, the obtained signal can be given as:

$M\left(k, N \cdot t_{\mathrm{a}}\right)=\iint F\left(z, T_{1}\right) \cdot \exp (i 2 \pi k z) \cdot\left[\exp \left(-\frac{N \cdot t_{\mathrm{a}}}{T_{1}}\right) \sin \alpha \cdot \cos ^{N-1} \alpha\right] d z d T_{1}$

with $k=\gamma g_{\max } \delta / m \pi . \quad g_{\max }$ is the maximum gradient intensity, $\delta$ is the duration of this gradient and $m$ is the step number of varying the gradient intensity from $-g_{\max }$ to $g_{\max }$. $z$ is the gradient direction which coincides with the cylindrical axis of the rock cores in this work.

To process this acquired 2D data, FT is firstly employed to transfer each 
phase pattern to be a imaging profile. Then the exponential decay data in each position is treated by using ILT to yield the resolved $T_{1}$ distribution function $F\left(z, T_{1}\right)$.

\subsubsection{Permeability profile}

The spatially resolved $T_{1}$ profile of rock cores carry several important petrophysical parameters which can be further employed to characterize the interior structure properties of the samples. In each resolved slice, the local $T_{1}$ distribution contains the pore size information. With the spatially resolved $T_{1}$ result from a calibration sample representing $100 \%$ pore volume, the signal intensity can be rescaled to be local porosity according to Eq. 3.4. Therefore, the corresponding permeability can be estimated using these two information according to Eq. 3.8, which is similar to the permeability curves estimated from relaxation time distributions for each layer formation in NMR well-logging interpretations as shown in Fig. 3.7. This consecutive calculation along the cylindrical axis of the sample will yield a local permeability value in analogue to the global permeability given by Eq. 3.8 as:

$$
\kappa_{\text {local }}^{i}=c \cdot\left(T_{1 l g m}^{i}\right)^{2}\left(\phi^{i}\right)^{4}
$$

where $c$ is an empirical factor related to the rock type. As suggested in the literatures, $c=1.6 \mathrm{mD} /(\mathrm{ms})^{2}$ for sandstone [21], and $0.1 \mathrm{mD} /(\mathrm{ms})^{2}$ for limestone $[22,235] . \phi^{i}$ is the porosity for $i^{t h}$ slice and $T_{1 \text { lgm }}^{i}$ is the logarithmic mean value of $T_{1}$ distribution from $i^{\text {th }}$ slice, which can be calculated based on Eq. 3.9. This approach from Eq. 5.4 is similar to the method as suggested in Ref. [236].

Eq. 5.4 reveals local permeability and therefore indicates the heterogeneous property of the studied material. However, this evaluation does not consider the connectedness between adjacent layers and might lead to a misinterpretation of some local permeable information. To illustrate this point, consider a two-layer porous medium model. One layer contains 
large pores but within a low porosity (for instance $T_{1 \operatorname{lgm}}=0.5 \mathrm{~s}$, and $\phi=$ 0.15). This layer represents some zones within vugs in a rock. Another one, by contrast, exists high population of small pores (for instance $T_{1 \mathrm{lgm}}=0.05$ $\mathrm{s}$, and $\phi=0.45$ ). This layer could stand for some tight zones within microcapillary/fractures in rock. The permeability values from these two layers are approximately equivalent $\left(\approx 150 \mathrm{mD}\right.$ with $c$ equal to $\left.1.6 \mathrm{mD} /(\mathrm{ms})^{2}\right)$ according to Eq. 5.4. However, the fluid can hardly flows along this medium because the significant difference of pore sizes between these two layers will largely exacerbate the pressure resistance for the flowing fluid. As a result, the internal connectedness should be considered in this local region.

Here, a factor $P$ is defined in order to take into account the local connectedness. It can be calculated from matching the adjacent relaxation distributions in neighboring slices (as shown in Fig. 5.3). This is based on the plausible assumption that pores will be well connected between two slices if these two $T_{1}$ distributions are highly similar to each other. On the contrary, differences (mismatches) of $T_{1}$ distributions would indicate changes and discontinuities in the pore structure most likely leading to increased transport resistances. As a result, the defined $P^{i, i+1}$ between the $i^{t h}$ and $(i+1)^{t h}$ slices is obtained by integrating the intersection between two adjacent slices and normalized by the union of their $T_{1}$ relaxation distributions:

$$
P^{i, i+1}=\frac{\sum_{j=a}^{b} \frac{\left[\operatorname{Min}\left(A_{j}^{i}, A_{j}^{i+1}\right)\right]^{2}}{A_{j}^{i} \cdot A_{j}^{i+1}}}{\operatorname{Length}\left[\operatorname{Union}\left(A^{i}, A^{i+1}\right)\right]} .
$$

Here $A_{j}^{i}$ is the $j^{\text {th }}$ normalized amplitude of the $T_{1}$ distribution in the $i^{\text {th }}$ slice, the sum of which along the $T_{1}$ axis is proportional to the local porosity of this slice. The index $a, b$ stand for the intersection boundaries of $T_{1}$ distributions from two adjacent slices shown in Fig. 5.3. The denominator in Eq. 5.5 is the union components number of these two $T_{1}$ distributions, which is used to normalize the intersection area of these two $T_{1}$ distributions calculated in the nominator of Eq. 5.5. From the definition of the parameter $P^{i, i+1}$ one can find that this connectedness (or penalty) factor is 1 in the 


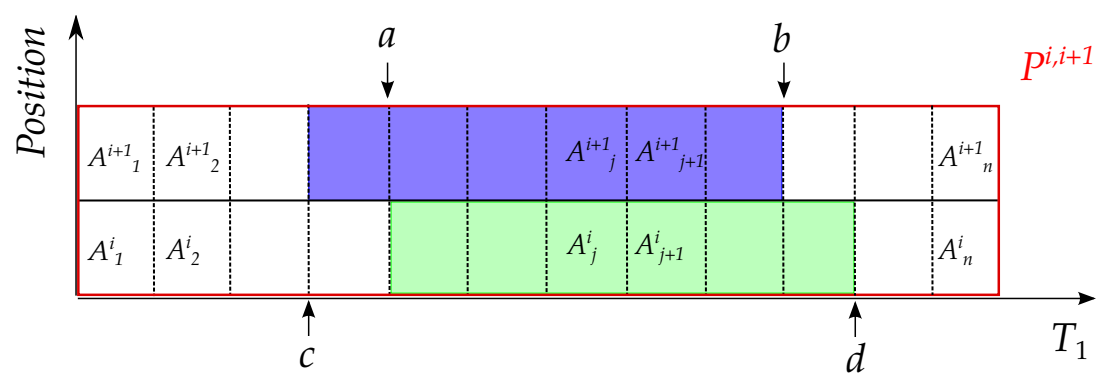

FIG. 5.3: A proposed model to calculate local permeability connected factor $P^{i, i+1}$. The purple and green blocks are the two adjacent $T_{1}$ distributions. The index $a$, $b, c$ and $d$ along $T_{1}$ domain represent the intersection and union boundaries of $T_{1}$ distributions from two adjacent slices.

case of identical distributions (total match) and is 0 in the non-matching distributions. Based on this, a local permeability within two adjacent slices considering the proposed connectedness factor can be defined as:

$$
\kappa_{\text {local }}^{i, i+1}=c \cdot T_{1 l g m}^{i} \cdot T_{1 l g m}^{i+1}\left(\phi^{i} \cdot \phi^{i+1}\right)^{2} \cdot P^{i, i+1} .
$$

\subsection{Experimental}

Two rock samples are selected and their permeability profiles are studied in this chapter. One is sandstone with porosity of $10.5 \%$ and brine permeability of $3 \mathrm{mD}$. Another one is a limestone core with porosity of $36.5 \%$ and permeability of $90 \mathrm{mD}$.

Before acquiring the spatially-resolved $T_{1}$ relaxation profiles, $2 \mathrm{D}$ imaging is used in order to visualize the interior structure of the rock samples. The NMR system consists of a cryogen free NMR Imaging system (Cryogenic Ltd, London, UK, $64 \mathrm{MHz}$ ) with homebuilt gradients and RF coil operated by a Kea console (Magritek Ltd, Wellington, NZ). A spin echo imaging technique is used with a RF pulse length of $30 \mu$ s and an echo spacing of $10 \mathrm{~ms}$. The number of pixels in the frequency and phase encoding directions are 128 each. The field of view is $150 \times 150 \mathrm{~mm}^{2}$.

The imaging results of the sandstone and limestone cores are given 
in Fig. 5.4. It is easy to visualize that the sandstone is fine-to-medium grained, red-brown quartz with a bedding (in-between dark slices) plane structure which is observable through the highlighted bands in the image. For the limestone core, a high heterogeneity can be observed from the photography by eyes and 2D MR imaging result. The lower intensity in the image corresponds to the large grain in the middle of the core.
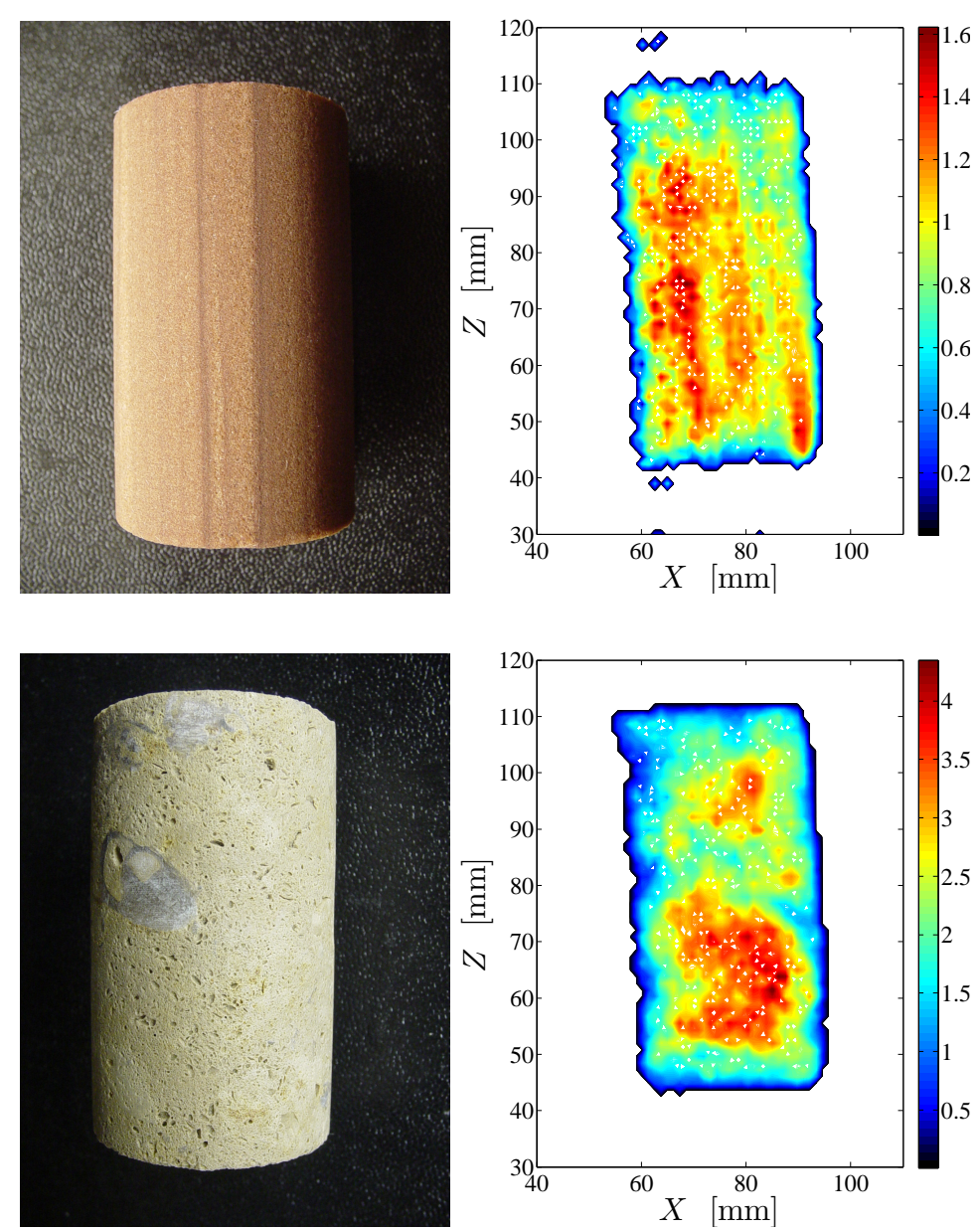

FIG. 5.4: Rock samples used for studying the permeability properties (left column) and their 2D MRI imaging results (right column). The imaging field of view is $150 \times 150 \mathrm{~mm}$ with a spatial resolution of $1.17 \mathrm{~mm}$. The unit of the MR imagines in the right column is $\mu \mathrm{V} / \mathrm{kHz}$. 
All fast encoding $T_{1}$ and spatially-resolved $T_{1}$ measurements are based on the rapid encoding given in Fig. 5.1 and are implemented on $2 \mathrm{MHz}$ Rock Core Analyzer (Magritek Limited Company). In the fast $T_{1}$ encoding experiment, the RF pulse length is $25 \mu \mathrm{s}$, the amplitude of $90^{\circ} \mathrm{RF}$ pulse is $-9.5 \mathrm{~dB}$, and the amplitude of $180^{\circ} \mathrm{RF}$ pulse is $-3.5 \mathrm{~dB}$, the amplitude of small tip angle $\alpha$ RF pulse is $-32.75 \mathrm{~dB}$, which corresponds to $5.3^{\circ}$. As denoted in Fig. 5.1, the number of FID is set to be 100. The acquisition time $t_{\mathrm{a}}$ is chosen to be $6.12 \mathrm{~ms}$ in sandstone and $26.6 \mathrm{~ms}$ in limestone. $\tau$ is $50 \mu \mathrm{s}$. Within a number of 64 scans and $6000 \mathrm{~ms}$ repetition time, the acquisition time is 6 min. Independently, inversion-recovery pulse sequence as discussed in Fig. 2.8 is applied to compare the $T_{1}$ results. $T_{\mathrm{W}}$ varies from $1 \mathrm{~ms}$ to $1500 \mathrm{~ms}$ in the sandstone and to $6000 \mathrm{~ms}$ in the limestone in 35 steps logarithmically. Within a number of 16 scans and $6000 \mathrm{~ms}$, the experimental time using this pulse sequence is approximately $63 \mathrm{~min}$.

As denoted in Fig. 5.2, a phase encoded gradient is applied to obtain the imaging pattern for spatially-encoded $T_{1}$ profiles based on the same experimental parameters adopted from the fast $T_{1}$ encoding experiment. The imaging resolution is set to be $1.5 \mathrm{~mm}$. The length of phase encoding gradient $\delta$ is $100 \mu \mathrm{s}$. Within a number of 64 phase gradient steps and $6000 \mathrm{~ms}$ repetition time, the acquisition time for the rock samples is approximately 6 hours.

\subsection{Results}

\subsubsection{Fast encoded $T_{1}$ data and distributions}

The measured $T_{1}$ data and inversion results of the sandstone are given in Fig. 5.5. In order to compare these two data sets, the measured data from the inversion-recovery pulse sequence is converted from the recovery to the attenuation pattern and only shown up to $0.9 \mathrm{~s}$. It is easy to observe that the data from fast encoding method (blue diamond) are comparable 
with the result from the traditional inversion-recovery (red triangle) pulse sequence. Furthermore, the $T_{1}$ distributions after ILT inversion as given in Fig. 5.5 (b) show good correspondence. Meanwhile, the measured $T_{1}$ data and inversion results of the limestone are given in Fig. 5.6. Again, when comparing the results from the inversion recovery and the fast encoded method, similar features in both the time domain and the $T_{1}$ ILT domain can be found.

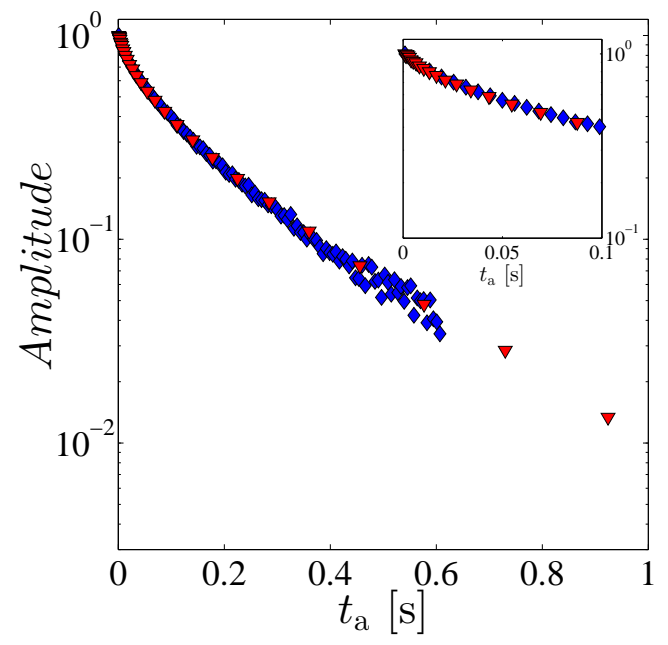

(a)

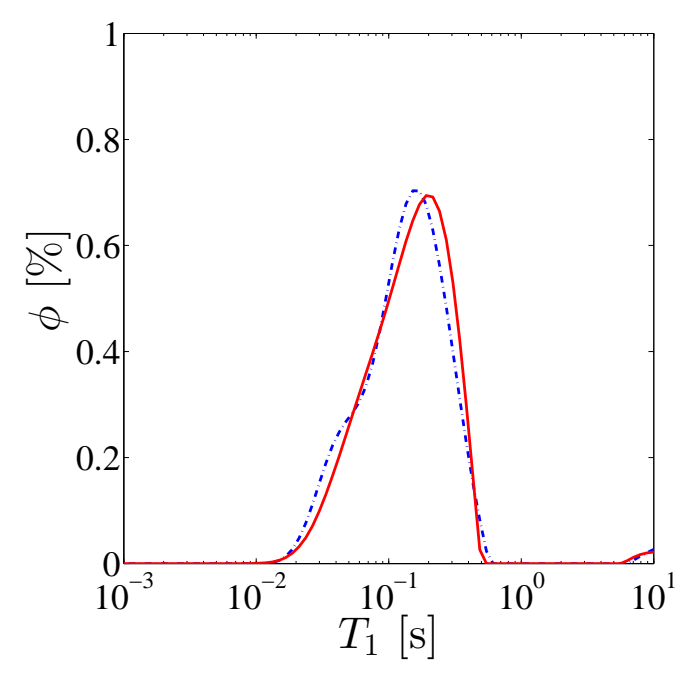

(b)

FIG. 5.5: (a) The comparison of the experimental data between fast encoding $T_{1}$ (blue diamond) and inversion-recovery (red triangle) techniques in the sandstone sample. The inset emphasizes the experimental data in the time scale ranging to $0.1 \mathrm{~s}$. (b) The comparison of the relaxation distributions between fast encoding $T_{1}$ (blue dashed) and inversion-recovery (red solid) techniques in the sandstone sample.

Note that the tails of the fast encoded $T_{1}$ datasets in these two cases are more noisy compared to the data from the inversion recovery pulse sequence. This is ascribed to more acquisition points used in the fast encoded $T_{1}$ measurements. However, the experimental results from sandstone and limestone samples suggest that this fast encoding method can yield reliable datasets and $T_{1}$ distributions, even in the field strength as low as at $2 \mathrm{MHz}$. 


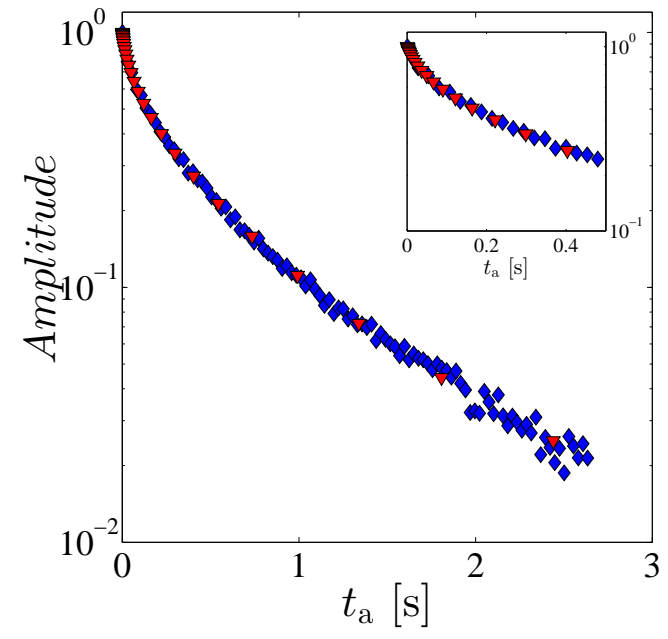

(a)

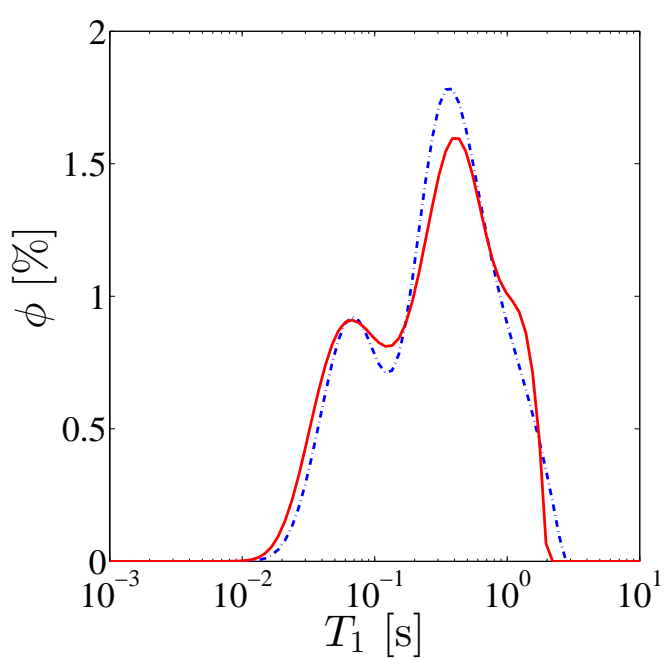

(b)

FIG. 5.6: (a) The comparison of the experimental data between fast encoding $T_{1}$ (blue diamond) and inversion-recovery (red triangle) techniques in the limestone sample. The inset emphasizes the experimental data in the time scale ranging to $0.5 \mathrm{~s}$. (b) The comparison of the relaxation distributions between fast encoding $T_{1}$ (blue dashed) and inversion-recovery (red solid) techniques in the limestone sample.

\subsubsection{Spatially-resolved $T_{1}$ profiles}

Spatially-resolved $T_{1}$ profiles of the sandstone and limestone samples are shown in Fig. 5.7. The projections in the top and right panels of the 2D maps represent the $T_{1}$ distributions and the porosity profiles along cylindrical axis, respectively. In order to obtain the absolute porosity, the signal intensity in each slice is rescaled by the experimental result from a calibration sample with $100 \%$ pore volume. The blue dashed lines in the right panels are the weighing porosities obtained before NMR experiments were performed. It is apparent that the projected porosity profiles, the values of which are comparable to the weighing porosities, characterize the local hydrogen distribution and therefore indicate the heterogeneity degree of rock samples. Besides that, the projected $T_{1}$ distributions of these two 
rock cores are compared with the non-spatially resolved $T_{1}$ results obtained from the fast encoding pulse sequence shown in Fig. 5.1. The projected $T_{1}$ distributions for both cases agree reasonably well, although there are some deviations which may be caused by more complex manipulation of the magnetization in spatially-resolved $T_{1}$ profile due to the extra $\pi$ pulse used in Fig. 5.2.

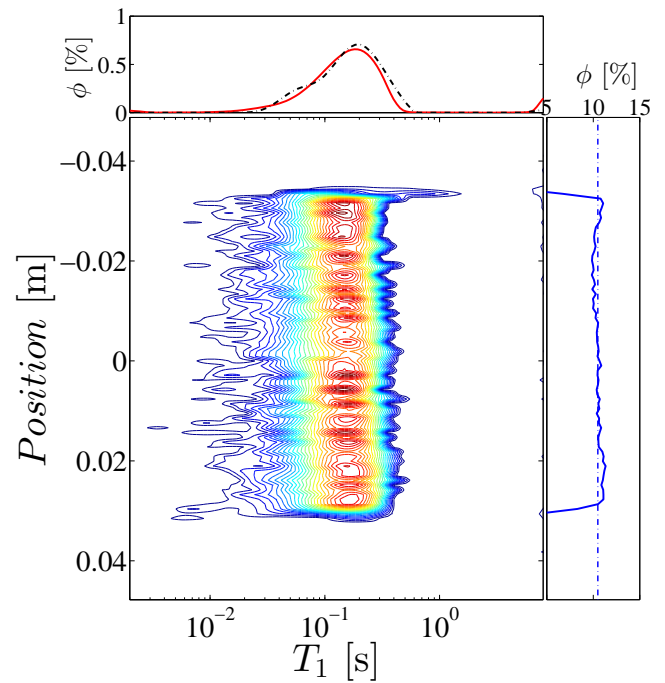

(a)

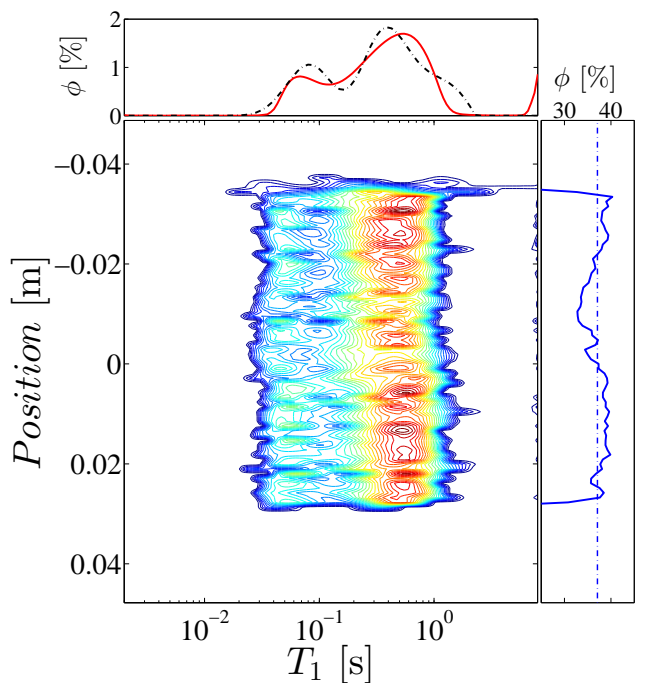

(b)

FIG. 5.7: Spatially resolved $T_{1}$ mapping in the sandstone (a) and limestone sample (b). The right and top projections of the spatially-resolved $T_{1}$ maps are the distributions of porosity along cylindrical axis and the $T_{1}$ distributions. The black dashed lines are the $T_{1}$ distributions acquired using the pulse sequence shown in Fig. 5.1. The blue dashed lines in the right panel represent the weighing porosity of each core plugs.

From the spatially-resolved $T_{1}$ distributions given in Fig. 5.7, it is easy to observe that the major distribution in the sandstone core sits in the $T_{1}$ relaxation range from $0.01 \mathrm{~s}$ to $0.3 \mathrm{~s}$, while expands from $0.03 \mathrm{~s}$ up to $2 \mathrm{~s}$ in the limestone. This implies a smaller pore length scales in the sandstone and more heterogeneous pore network in the limestone core. By comparing these two relaxation profiles along the cylindrical axis of cores, 
the sandstone appears to be relatively uniform while the limestone reveals more fluctuation and particularly a dip zone in the middle of core. This indicates that this core has a more heterogeneous solid matrix as compared to the sandstone. Notably, this conclusion agrees with the rock core imaging results given in Fig. 5.4.

\subsubsection{Permeability profiles}

Using the obtained $T_{1}$ profiles and the model defined in Section 5.1.3, two local permeability profiles of these two rock samples, with and without considering the connectedness factor $P^{i, i+1}$ can be calculated based on Eq. 5.6 and Eq. 5.4 and are given in Fig. 5.8. The curves are the permeability profiles calculated with (blue) and without (pink) considering $P$, respectively. Black dot lines represent the permeability values from brine flowing measurements.

Analogue to a series circuit in electronics, the rock slice with the worst permeability determines the moveability of the saturating fluid in the rock core. This can be seen from the comparison of the lowest value of the obtained permeability profile along the cylindrical axis with the brine permeability in both rock cores in Fig. 5.8. Note that the permeability profiles which are calculated using the connectedness factor $P$ as defined in Eq. 5.5 approaches closer to the reference brine permeability line than the curve without considering $P$.

In order to quantitatively evaluate these results, the upscaling technique is utilized to obtain an averaged permeability from its profile [237-239]. The calculated results are denoted using blue and pink dashed lines in Fig. 5.8. Here, the averaged permeability of the sandstone with and without considering the connectedness factor are $3.21 \mathrm{mD}$ and $6.83 \mathrm{mD}$, respectively, compared to its brine permeability of $3 \mathrm{mD}$. Similarly, the averaged permeability of the limestone with and without considering the connectedness factor are $132 \mathrm{mD}$ and $151 \mathrm{mD}$, respectively, compared to its brine per- 


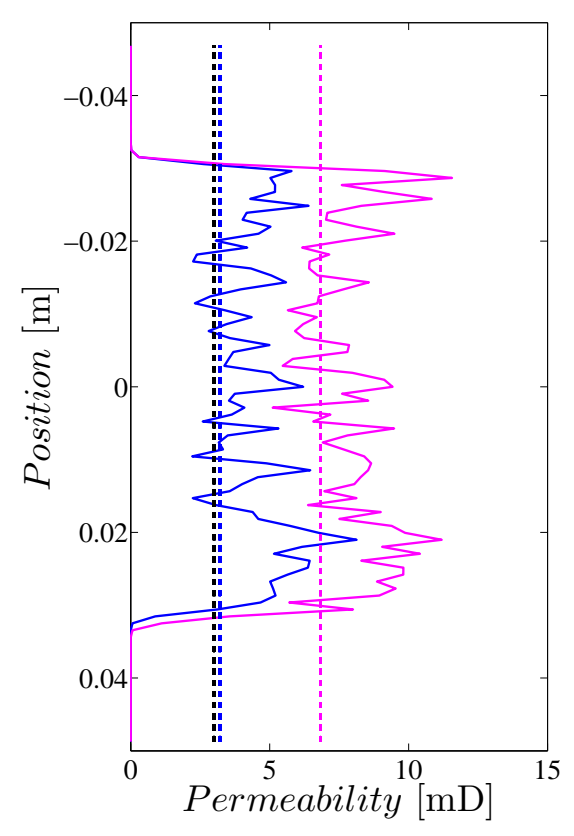

(a)

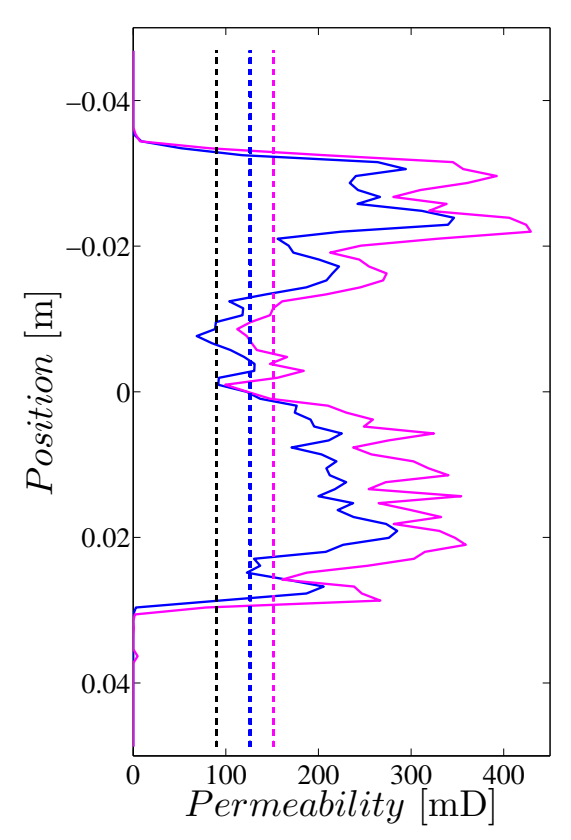

(b)

FIG. 5.8: Permeability profiles estimated in the sandstone (a) and limestone cores (b). The blue and pink curves are the permeability profiles calculated with and without considering the proposed penalty factor, respectively. Black dashed lines represent the brine-permeability measurement values. Blue and pink dashed lines are the renormalization of permeability profiles with and without considering the connectedness factor $P^{i, i+1}$.

meability of $90 \mathrm{mD}$. Both cases indicate that the averaged permeability values from considering the connectedness factor $P$ are closer to the brine permeabilities as compared to the values without considering the connectedness factor. As a result, a better description of the permeable properties can be obtained if a connectedness factor $P$ according to Eq. 5.5 between two adjacent resolved slices is taken into account.

Moreover, by comparing the rock core imaging results given in Fig. 5.4, with the local permeability profiles it is evident that the spatial heterogeneities of rocks along their cylindrical axis are reflected well by both 
methods, thus making 1D spatially resolved relaxation maps as a source for the permeability profile an available alternative to 2D imaging. 


\section{Chapter 6}

\section{Multi-phase Fluid Flooding}

In this chapter, an overburden system was utilized to pressurize rock cores at required temperatures and pressures. Furthermore, spatially resolved NMR techniques were combined with relaxation time $\left(T_{1}\right.$ and $\left.T_{2}\right)$ and relaxation-diffusion correlation $\left(D-T_{2}\right)$ experiments and implemented on a low-field NMR rock core analyzer. The property and distribution of oil saturating rock core were studied at different temperatures before flooding experiment. Subsequently, the conditions of secondary oil recovery were investigated by flooding water through an oilbearing rock plug [240]. To our knowledge, this is for the first time to combine spatially-resolved NMR relaxometry/diffusometry technique with overburden system, to monitor the multi-phase fluid transport in rock core at different confining conditions.

\subsection{Hardware configuration}

\subsubsection{Overburden system}

A commercial $2 \mathrm{MHz}$ NMR analyzer equipped with a shielded 1D gradient coil (Magritek, Ltd.) was used to acquire NMR data in this work [89]. The RF probe has a dimension of $10 \mathrm{~cm}$ length and $5.4 \mathrm{~cm}$ diameter. This setup is complemented with a Daedalus Innovations overburden 
rock holder (see in Fig. 6.1), which maintains required temperatures and pressures for the rock plug under study [193].

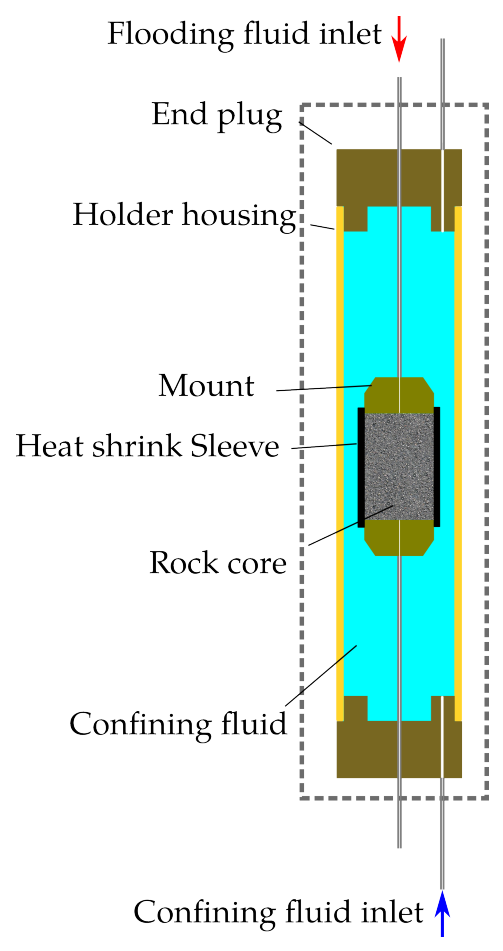

(a)

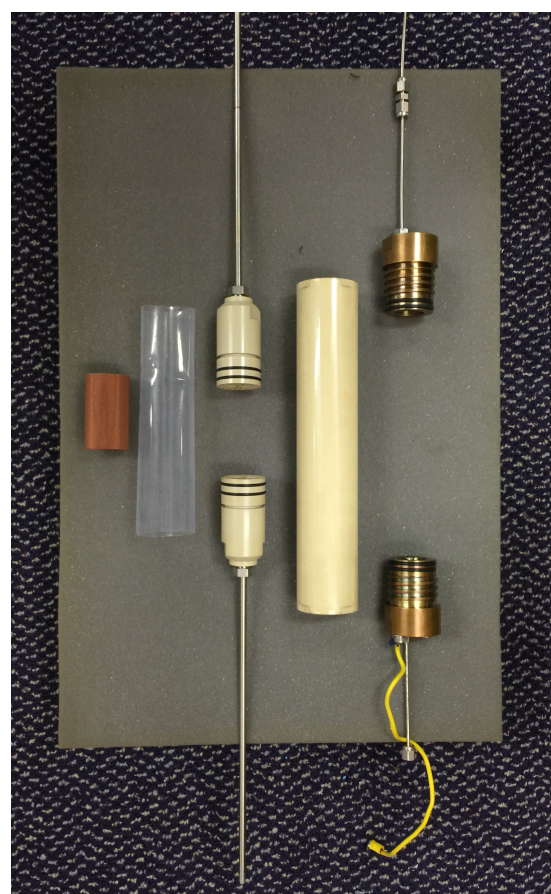

(b)

FIG. 6.1: (a) Schematic of overburden rock holder. Red and blue arrows indicate the flow direction of flooding fluid and confining fluid, respectively. (b) The individual components of the overburden rock holder. The parts from left to right columns are rock core, heat shrink sleeve, mount, holder ceramic housing and brass end plugs. The yellow wire in the bottom end plug is used to ground the rock holder electronically.

As shown in Fig. 6.1, the rock core is mounted in the rock holder before performing the experiments. It is placed between two PEAK mounts first, wrapped by heat shrink sleeve, and then loaded in the chamber of the overburden holder. Two brass plugs are screwed in each end of the ceramic holder housing to seal the overburden cell. It is worth noting that the materials of the rock holder, especially the section close to the detection volume of the NMR system, are chosen to be non-magnetic as to avoid 
interference with the NMR experiments.

Subsequently, the cell holding the rock plug is loaded in the bore of NMR system and is connected to two external circuits. These two circuits will provide pressurized fluid flooding through rock samples (left plumbing in Fig. 6.2), and the confining pressure and temperature for the rock plug (right loop in Fig. 6.2), respectively.

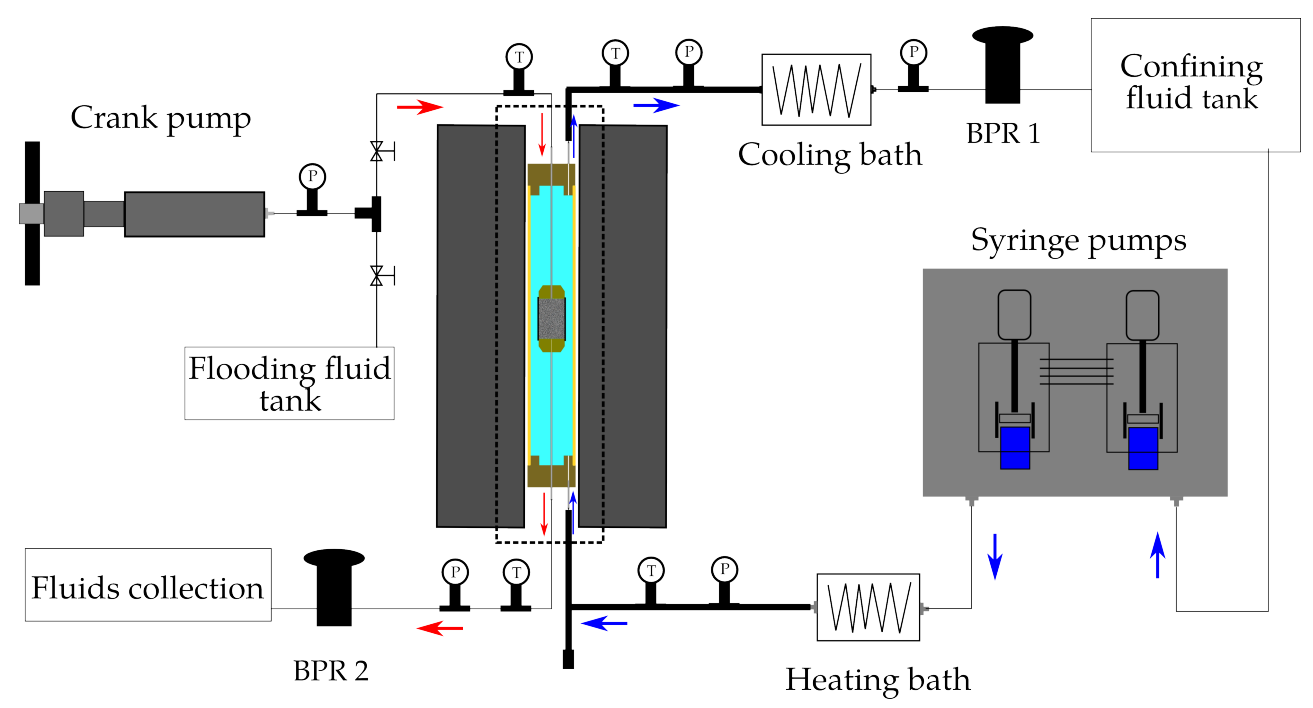

FIG. 6.2: Schematic of overburden rock holder combining with low-field NMR system for high pressure and temperature experiments. Red and blue arrows indicate the flow direction of flooding fluid and confining fluid, respectively. The bold line in the confining part represents the plumbing with tubing insulation. P in the circuit stands for pressure gauge and $\mathrm{T}$ for thermocouple.

To achieve the desired confining temperature and pressure for the rock plug, two ISCO syringe pumps are used to drive confining fluid flow as shown in Fig. 6.2. There are two requirements the confining fluids needs to meet. Firstly, the fluid should undertake the function for heat transfer at high temperatures. Secondly, it should not contain hydrogen. Accordingly, perfluro polyether (PFPE, $\mathrm{C}_{50} \mathrm{~F}_{102} \mathrm{O}_{16}{ }^{1}$ ) is chosen as the confining fluid to circulate in the plumbing and rock holder chamber. A heating exchange

\footnotetext{
${ }^{1}$ Molecular weight: 1020 , density: $1.82 \mathrm{~g} / \mathrm{mL}$, boiling point: $230^{\circ} \mathrm{C}$, thermal conductivity: $0.065 \mathrm{~W} /(\mathrm{m} \cdot \mathrm{K})$, dynamic viscosity: $8 \mathrm{cP}$.
} 
coil immersed in the heating bath after the syringe pump ensures the required temperature of PFPE before entering the pressure cell. Another exchange coil in the chiller will cool down the confining fluid after the cell. Before the confining fluid is flowing back to the reservoir, a back pressure regulator (BPR 1) is implemented which controls the pressure surrounding the rock core. On-site pressures and temperatures are logged to monitor the confining conditions of the loaded rock core real-time. A rupture disc is used to protect the plumbing from too high pressures. Furthermore, a highpressure crank pump is connected to the inlet end of the flooding channel of the overburden rock holder to provide flooding conditions. Water can be imbibed and then pressurized through the rock core in this work. Another back pressure regulator (BPR 2) is used to control the flooding pressure.

Subsequently, a rock sample saturating with diesel is loaded in the pressurized cell and the pressure and temperature data from different sites are logged after the entire system reaches thermal equilibrium. Experimental test data as shown in Table. 6.1 confirm the compatibility between operational NMR system and the overburden system. Note that the pressure drop in the confining circulation plumbing rises up with an increasing flow rate as driven by the syringe pump. On the contrary the pressure drop decreases with increasing of the temperature, which is due to the viscosity reduction of the heated confining fluid at higher temperatures. The temperature of the RF coil can rises up to $70^{\circ} \mathrm{C}$, which needs to be kept in mind for the influence to the sensitivity of the RF coil. The temperature gradient of the sample is $3.4^{\circ} \mathrm{C}$ for a flow rate of $150 \mathrm{~mL} / \mathrm{min}$ and $200^{\circ} \mathrm{C}$ of the heating bath temperature. Under this circumstance, the convection current of this rock core is evaluated by using the theory of Rayleigh-Bernard instability considering its permeability value. The Rayleigh number in this case is calculated to be approximately $10^{-6}$, which is quite small as compared to the criteria Rayleigh number of 1708 [241]. Thus, there should not be strong convection effects in this rock core experiment. Consequently, this set-up satisfies our requirements and will be the experimental condition 
for temperature dependent experiments under high pressure.

Table 6.1: Pressure and temperature data monitored at different sites. $Q$ is the flow rate which can be precisely controlled by ISCO syringe pump. For different temperature traces, 1 is for heating bath, 2 for magnet, 3 for RF coil, 4 for the confining fluid after heating bath, 5 for the confining fluid after cooling bath, 6 from rock core top, 7 from rock core bottom and $\Delta$ stands for the temperature gradient across the rock sample. $\Delta \mathrm{P}$ is the pressure drop across the entire confining circulation plumbing.

\begin{tabular}{cccccccccc}
\hline $\mathrm{Q}$ & \multicolumn{8}{c}{ Temperature $\left({ }^{\circ} \mathrm{C}\right)$} & $\Delta \mathrm{P}$ \\
\cline { 2 - 9 }$(\mathrm{mL} / \mathrm{min})$ & 1 & 2 & 3 & 4 & 5 & 6 & 7 & $\Delta$ & $(\mathrm{psi})$ \\
\hline 50 & 100 & 26.2 & 39.7 & 83.6 & 17.5 & 63.9 & 72.0 & 8.1 & 83 \\
50 & 150 & 25.9 & 48.8 & 131.1 & 17.4 & 96.2 & 101.2 & 5.0 & 74 \\
50 & 200 & 28 & 61.3 & 175.5 & 18.0 & 124.5 & 129.8 & 5.3 & 68 \\
\hline 100 & 100 & 25.9 & 41.7 & 87.1 & 19.1 & 73.3 & 78.9 & 5.6 & 173 \\
100 & 150 & 26.4 & 53.6 & 131.4 & 20.6 & 110.3 & 113.7 & 3.4 & 149 \\
100 & 200 & 31.6 & 72.7 & 179.8 & 25.7 & 146.6 & 151.9 & 5.3 & 126 \\
\hline 150 & 100 & 24 & 40.1 & 83.2 & 20.3 & 77.0 & 79.1 & 2.1 & 279 \\
150 & 150 & 26.5 & 54.0 & 125.6 & 30.3 & 112.3 & 115.2 & 2.9 & 212 \\
150 & 150 & 29.4 & 56.6 & 126.2 & 32.0 & 113.0 & 116.4 & 3.4 & 207 \\
150 & 200 & 30 & 73.6 & 178.4 & 49.9 & 154.3 & 157.7 & 3.4 & 165 \\
\hline
\end{tabular}

\subsubsection{Sensitivity for NMR measurements}

In order to examine the sensitivity and stability of the NMR analyzer with the implemented overburden system at different temperature ratings, a pure fluid, tetradecane, is placed in a Teflon holder and loaded in the pressured cell for test measurements (see in Fig. 6.3 (a)). According to Boltzmann distribution given in Eq. 2.4 and Curie Law in Eq. 2.9, the measured signal amplitude will inherently decrease with raising the sample temperatures. Therefore, a CPMG pulse sequence is applied to obtain the signal at different temperatures. The initial amplitude and the noise level of CPMG echo trains is given in Fig. 6.3 (b)). 


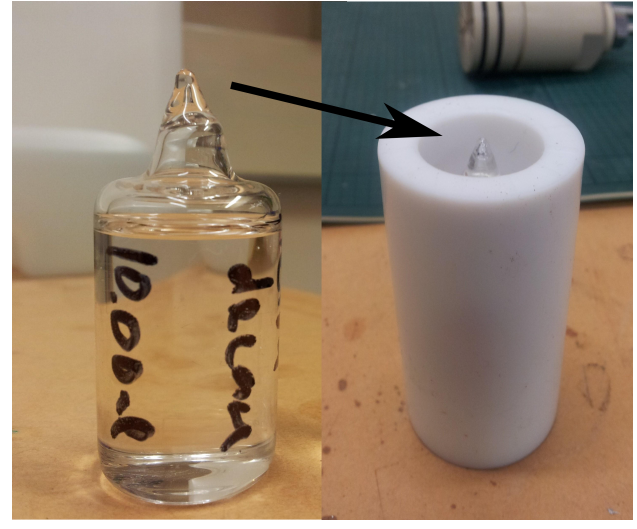

(a)

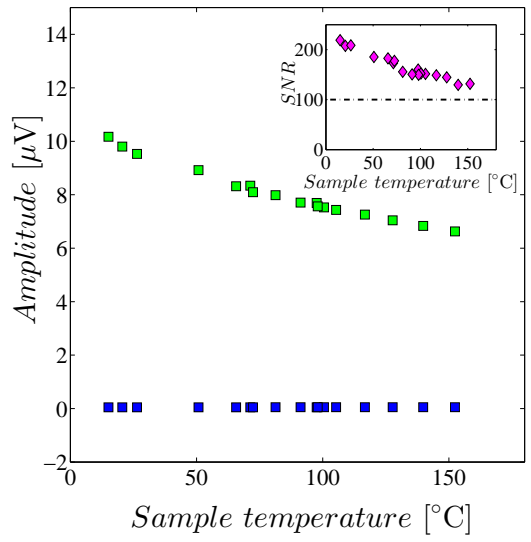

(b)

FIG. 6.3: (a) Sensitivity test of the entire system. A pure fluid, tetradecane $\mathrm{CH}_{3}\left(\mathrm{CH}_{2}\right)_{12} \mathrm{CH}_{3}$ is used and loaded in a Teflon holder. The boiling point of tetradecane is $253^{\circ} \mathrm{C}$. (b) The initial amplitudes and noise level of the CPMG signals at different temperatures. The SNR (see inset) is still above 100 using 4 scans at $155^{\circ} \mathrm{C}(\mathrm{b})$.

Note that the amplitudes of CPMG signals attenuate with increasing of the sample temperature, which can be explained by Eq. 2.9. The Root Mean Square (RMS) of the noise data is relatively constant along the horizontal axis, which demonstrate the reliability of RF coil in this case. Furthermore, by calculating the SNRs of the CPMG signals shown in the inset of Fig. 6.3, we prove that data acquisition is of high quality using only 4 scans even up to $155^{\circ} \mathrm{C}$ sample temperature. Therefore, the sensitivity and stability of the NMR analyzer is well established with this configuration.

\subsection{Methodology}

\subsubsection{Spatially-resolved relaxation profiles}

In the context of petrophysical analysis using low-field NMR measurements, the longitudinal relaxation $T_{1}$ and transverse relaxation $T_{2}$ are mostly used to study fluid-saturated rock cores. This may provide informa- 
tion on the pore volume and pore size distributions. The 1D phase encoded imaging technique combined with $T_{2}$ relaxation measurements, as shown in Fig. 6.4, can yield spatially resolved $T_{2}$ profiles [77, 242-244]. The first period covered by $T_{\mathrm{E} 1}$ is the phase encoded imaging part of the experiment, providing the 1D image (profile). The following section, consisting of the $180^{\circ}$ pulse train acquires the NMR echo decay, which is determined by the transverse relaxation $T_{2}$. Similarly, as given in Fig. 5.2, spatially resolved $T_{1}$ profiles can be obtained by measuring $T_{1}$ using rapid acquisition schemes as suggested in $[32,233]$, again in conjunction with the 1D imaging method.

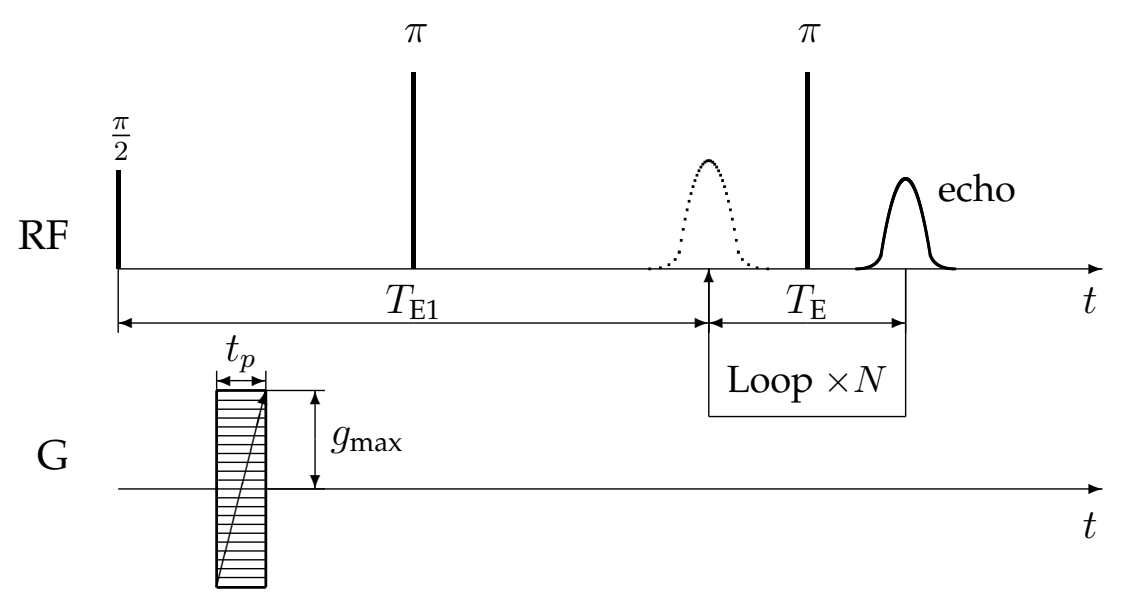

FIG. 6.4: NMR pulse sequence for spatially resolved $T_{2}$ profiles by using phase encoded methods [242]. $g$ is the imaging gradient which has variable intensities with a duration of $t_{p} . T_{\mathrm{E}}$ is the echo spacing during the acquisition of the $T_{2}$ measurement.

The acquired data for spatially resolved $T_{2}$ experiments can be expressed as:

$$
M\left(k, N T_{\mathrm{E}}\right)=\iint F\left(z, T_{2}\right) \cdot \exp (i 2 \pi k z) \cdot \exp \left(-\frac{N T_{\mathrm{E}}}{T_{2}}\right) d z d T_{2}
$$

while the pulse sequence for the spatially resolved $T_{1}$ imaging is shown in Fig. 5.2 and the acquired data expression is already given Eq. 5.3 of Chapter 5. The $k$ vector is expressed as $\gamma g t_{p} / m \pi . g_{\max }$ is the maximum gradient intensity, $t_{p}$ is the duration of this gradient and $m$ is the step 
number of varying the gradient intensity from $-g_{\max }$ to $g_{\max }$. $z$ is the gradient direction which coincides with the cylindrical axis of the rock cores in this work.

Both spatially resolved $T_{1}$ and $T_{2}$ imaging methods yield two-dimensional datasets and a two-step data process is needed to extract the spatial relaxation distribution function $F\left(z, T_{1}\right)$ or $F\left(z, T_{2}\right)$. Firstly, Fourier transform is employed to transform the encoded phase information into positions, returning the fluid content in each resolved slice (pixel). A customized ILT algorithm is then applied to obtain the spatial resolved relaxation distribution function [6]. It is worth noting that $F\left(z, T_{1}\right)$ and $F\left(z, T_{2}\right)$ are akin to the curves from NMR well logging operated in the oilfield, where the sensor is pulled from the down-hole up to the surface and provide relaxation distributions from each vertical layer in the formation.

\subsubsection{Spatially-resolved $D-T_{2}$ correlation maps}

Besides relaxation measurements, diffusion coefficients of fluids can be measured by NMR techniques [40,49]. This allows one to identify different fluid types and quantify fluid saturation in rock samples. In this case, 2D $D-T_{2}$ NMR correlation technique provides substantial information in rock core analysis, such as fluid typing and wettability indication in multi-phase experiments. Furthermore, spatially resolved $D-T_{2}$ distribution provides the aforementioned information along a certain core axis and potentially indicates progress of oil recovery or monitors the saturation level of rock cores [79]. While incorporating the 2D relaxation-diffusion correlation experiment with the 1D imaging technique, thus making the experiment $3 \mathrm{D}$, the entire experimental time should be taken into account for practical reasons. Therefore, the frequency encoding method should be preferred because of a similar experimental time as compared to the 2D correlation experiment without spatial resolution.

The pulse sequence for spatially resolved $D-T_{2}$ distribution is shown in 
Fig. 6.5 and the signal decay is expressed as:

$$
\begin{aligned}
M\left(k, G, N T_{\mathrm{E}}\right) & =\iiint F\left(z, D, T_{2}\right) \cdot \exp (i 2 \pi k z) \\
& \cdot \exp \left(-\gamma^{2} \delta^{2} G^{2} D(\Delta-\delta / 3)\right) \cdot \exp \left(-\frac{N T_{\mathrm{E}}}{T_{2}}\right) d z d T_{2}
\end{aligned}
$$

where $k=\gamma g t_{p} / 2 \pi . \Delta$ is the diffusion observation time and $\delta$ is the duration of the gradients for diffusion encoding. $N$ is the number of the acquired echoes. The result is a 3D data matrix acquired during the spatially resolved $D-T_{2}$ experiment. To obtain the final map, Fourier transform is performed on each acquired echo to obtain the spatial imaging profile. Then 2D ILT algorithm is applied subsequently for the $2 \mathrm{D}$ exponential data decay in each slice, in order to extract the local $D-T_{2}$ distribution function $F\left(z, D, T_{2}\right)$ [95].

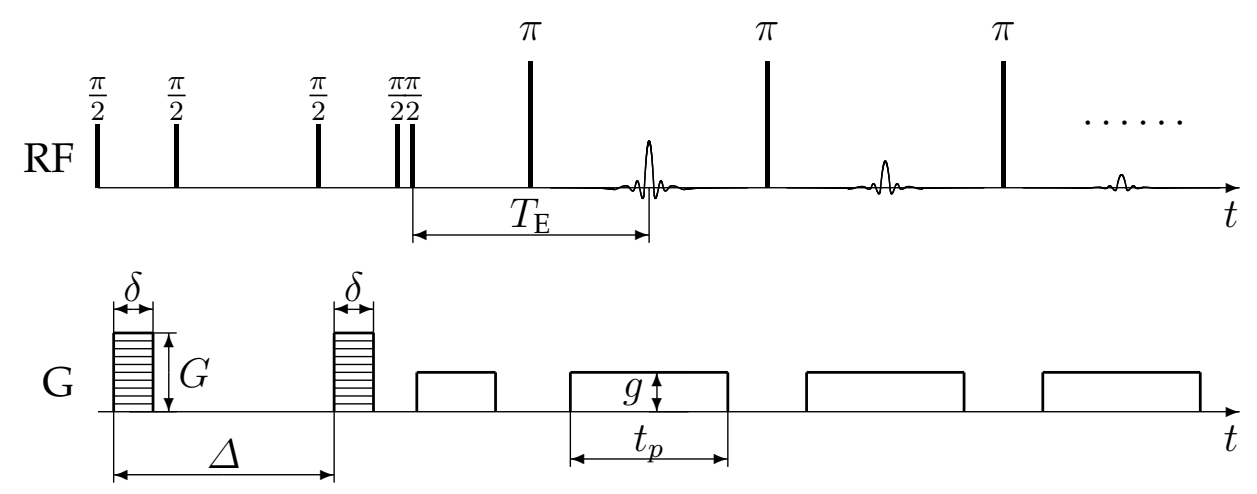

FIG. 6.5: NMR pulse sequence for spatially resolved $D-T_{2}$ map, details are shown in [79]. $\Delta$ is the diffusion observation time and $\delta$ is the gradient duration. $G$ and $g$ are the gradients for encoding diffusion coefficient and position, respectively. $T_{\mathrm{E}}$ is the echo spacing and $t_{p}$ is the echo acquisition time.

\subsection{Experimental}

Samples. An Edwards Brown limestone core was used here to study fluid presence and multi-phase fluid flooding at different temperatures. 
The core porosity is $36.5 \%$ and the permeability is $90 \mathrm{mD}$. The rock core has a dimension of $6.3 \mathrm{~cm}$ length and $3.84 \mathrm{~cm}$ diameter. Before the experiment, the core plug was dried in an oven at $60^{\circ} \mathrm{C}$ for $48 \mathrm{~h}$, until no hydrogen signal was detected in NMR measurements. Afterwards it was saturated with diesel (Hydrogen index HI=1.023). In order to recall the properties of this rock cores, its 1D imaging profile and pore length scale are given in Fig. 6.6 (b) and (c). The heterogeneity of the rock sample can be recognized from the comparison of the acquired porosity profile and weighing porosity indicated by a dashed line. Large grains existing close to the plug middle might lead to the section with reduced porosity. This rock plug has a wide range of pore length from approximately $1 \mu \mathrm{m}$ to $100 \mu \mathrm{m}$, indicating a highly heterogeneous pore system.

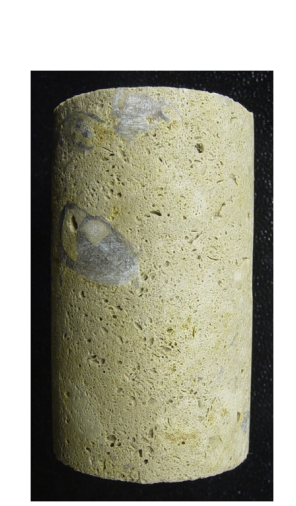

(a)

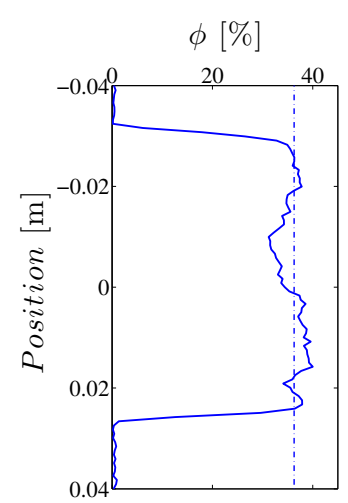

(b)

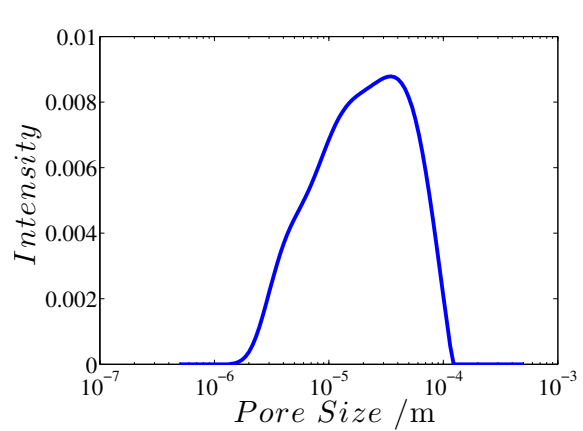

(c)

FIG. 6.6: Edwards Brown plug used in this work (a), its porosity profile (b) determined by 1D imaging, and its pore length scales (c) determined by DDIF technique [157].

Basic parameters in NMR experiments. The duration of 90 and $180^{\circ}$ pulses in the NMR experiments were kept to be $25 \mu \mathrm{s}$ (to ensure the same RF-pulse frequency bandwidth) while the amplitudes were adjusted accordingly. The amplitude of $90^{\circ}$ and $180^{\circ}$ RF pulses are $-9.5 \mathrm{~dB}$ and -3.5 $\mathrm{dB}$, respectively. $T_{1}, T_{2}$ and $D-T_{2}$ experiments were performed as routine measurements for determining the global properties of the fluid in the rock 
core. Relaxation time $T_{2}$ distributions were determined using the CPMG [34] pulse sequence with an echo spacing of $150 \mu$ s and 12000 echoes. A dataset with $\mathrm{SNR}=450$ was acquired within a 4 -scan measurement lasting $45 \mathrm{~s}$. In the fast encoding $T_{1}$ measurement, the small tip angle $\alpha$ was set to be $5.3^{\circ}$ and the number of small tip angle pulse is 250 . The acquisition time $t_{\mathrm{a}}$ was optimized to be $25.6 \mathrm{~ms}$. The entire experimental time was 6 min using 32 scans. $D-T_{2}$ correlation distributions were obtained with a gradient duration $\delta=5 \mathrm{~ms}$ and diffusion observation time $\Delta=40 \mathrm{~ms}$. The maximum gradient $G$ for encoding diffusion was up to $0.4 \mathrm{~T} / \mathrm{m}$ in 40 steps linearly. The echo spacing was $150 \mu$ s and the number of echoes was 12000.

Before flooding: static measurement. Because of the smaller impact of $T_{2}$ relaxation during the imaging encoding period, the phase-encoded $T_{2}$ method as shown in Fig. 6.4 was used to extract spatially resolved $T_{2}$ profiles in the case of static fluid measurement. The spatial resolution was $1.5 \mathrm{~mm}$ and the number of imaging gradients $m$ was 64 . The echo spacing in the first stage $T_{\mathrm{E} 1}$ in Fig. 6.4 was set to be $500 \mu \mathrm{s}$ and the duration of the phase gradient was $100 \mu \mathrm{s}$. The echo spacing in the second stage was set to be $150 \mu$ s and the number of echoes was 12000 . With an 8 -scan measurement for each imaging gradient step, phase-encoded $T_{2}$ profile datasets have been acquired within $1.5 \mathrm{~h}$. For phase encoded $T_{1}$ measurement, the spatial resolution was $3 \mathrm{~mm}$ and the number of imaging gradients $\mathrm{m}$ was 32 . The data can be acquired within $3.5 \mathrm{~h}$ with a 32-scan measurement for each imaging gradient step.

Before flooding, the confining pressure was set to be 1000 psi by adjusting BPR 1 in the confining circuit. To study the temperature-dependence of oil properties and distribution in rock plug, the temperatures of rock sample were set to be $25^{\circ} \mathrm{C}, 55^{\circ} \mathrm{C}$ and $80^{\circ} \mathrm{C}$ with a constant flow rate of $150 \mathrm{~mL} / \mathrm{min}$ for the confining fluid PFPE heated in the heating bath. During this measurement, the inlet and outlet of flooding channel were both switched off to avoid the saturated oil being driven out while maintaining the confining pressure. 
Flooding measurements. In the flooding experiment, to obtain spatially resolved $D-T_{2}$ distribution efficiently, the frequency encoded imaging method as shown in Fig. 6.5 was used. The experimental parameters used here have been optimized to ensure comparable results obtained from frequency encoded $T_{2}$ profiles as compared to phase encoded $T_{2}$ method. The intensity of the frequency gradient $g$ was chosen to be $12 \mathrm{mT} / \mathrm{m}$. The number of acquired data points for each echo was 256 and the dwell time was $2 \mu \mathrm{s}$. This ensured the acquisition of the full echo with in the read gradient $g$. The echo spacing with $T_{\mathrm{E}}=800 \mu$ s was chosen accordingly to suit the acquisition of the full echo. The number of echoes was 3000 . For the acquisition in the diffusion dimension, the gradient duration $\delta$ was 5 $\mathrm{ms}$ and the diffusion observation time $\Delta$ was $40 \mathrm{~ms}$. The gradient $G$ for diffusion encoding varied up to $0.4 \mathrm{~T} / \mathrm{m}$ in 12 steps linearly. The experimental time was 20 mins using 16 scans.

To investigate the oil recovery procedure, water was injected into the oil-saturated rock core confined in the overburden cell. During the flooding experiment, to avoid water bypassing the core and travel between the core sample and confining fluid, the overburden pressure was set to be at least 300 psi higher than the inlet pressure of the crank pump. Therefore, the pressure before BPR 1 was kept to be 1000 psi and the initial pressure for crank pump was 700 psi. The pressure before BPR 2 was set to 500 psi. The entire flooding procedure is carried out in small individual steps as indicated on the horizontal axis in Fig. 6.11. The crank pump is suspended after each flooding step. Spatially resolved $D-T_{2}$ NMR measurements are carried out after there are no reading changes from these two pressure gauges between the confined rock core. After that further flooding steps were applied in the same manner until the oil signal (as estimated from the spatially resolved $D-T_{2}$ maps) settled to a new stationary value. Finally, this was then followed by an increase of the sample temperature and a next set of flooding and NMR measurements was acquired, thus revealing the temperature dependence of the water-flooding-oil process in the rock core. 
Three experimental temperatures, $25^{\circ} \mathrm{C}, 55^{\circ} \mathrm{C}$ and $80^{\circ} \mathrm{C}$, have been adopted in this work.

\subsection{Results}

\subsubsection{Porosity profile and fluid distribution}

Before spatially resolved measurements were performed, $T_{2}, T_{1}$ and $D-T_{2}$ distributions at three temperatures at 1000 psi were acquired (Fig. 6.7). $T_{2}$ distributions at the three temperatures indicate two components of oil in the pore space (light component corresponds to larger $T_{2}$ value while heavy components has a smaller $T_{2}$ value). With increasing temperature, $T_{2}$ distribution gradually shifts towards longer relaxation time, which implies a decrease of oil viscosity $[245,246]$. When the sample confining temperature was increased from $55^{\circ} \mathrm{C}$ to $80^{\circ} \mathrm{C}$, the heavy component was less shifted as compared to the light counter-part, which might be caused by a weaker temperature dependence as compared to light one. Likewise, $T_{1}$ results obtained from rapid encoding method (shown in Fig. 5.1) progressively increase with heating up the rock core, which again suggest the decrease of the oil viscosity.

The diffusion-relaxation time correlation distributions of this oil-saturated rock plug under different temperatures were obtained and presented in Fig. 6.8. Because of relaxation effects during the diffusion observation time, the heavy component with short $T_{2}$ relaxation time in the correlation experiments were missing compared to the 1D relaxation results. Nevertheless, a reasonable prediction of the oil viscous properties is still possible since the light component predominates this oil as shown in 1D $T_{2}$ spectra. Meanwhile, these 2D distributions exhibit a strong correlation between diffusion coefficient $D$ and relaxation time $T_{2}$, a feature that is similar to that in bulk oil. It indicates non oil-wet status for this rock plug. This is probably ascribed to a thin water film absorbed at the pore walls that have 


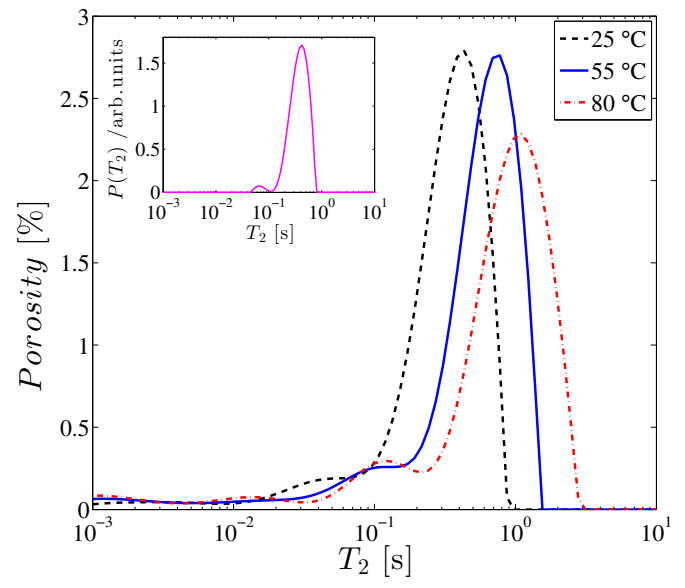

(a)

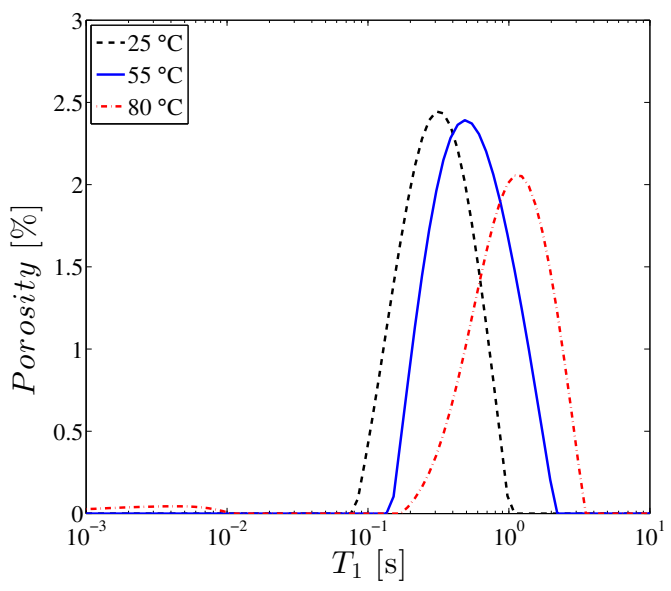

(b)

FIG. 6.7: (a) Transverse relaxation $T_{2}$ and (b) longitudinal relaxation $T_{1}$ distributions of oil saturating rock at $1000 \mathrm{psi}$. The dash, solid and dot dash lines represent the distributions at $25^{\circ} \mathrm{C}, 55^{\circ} \mathrm{C}$ and $80^{\circ} \mathrm{C}$ respectively. The regularization factor during the inversion is 1 in $T_{2}$ and 33 in $T_{1}$ data process. The $T_{2}$ distribution of bulk diesel at $25^{\circ} \mathrm{C}$ is given in the inset of (a).

not entirely dried out during the sample preparation. This amount of water, although not observable during the experiment, results in the wettability of this rock plug. The distributions shift along the hydrocarbon line even at temperatures of up to $80^{\circ} \mathrm{C}$, proves the decrease of oil viscosity. The shape of the $D$ and $T_{2}$ distributions remain almost the same, indicating a nearly stable composition of the oil in the pore space within the chosen temperature range [246].

Spatially resolved $T_{1}$ and $T_{2}$ profiles at $25^{\circ} \mathrm{C}$ are presented in Fig. $6.9 \mathrm{a}$ and $b$, respectively. Similar features, such as the heterogeneity of porosity profile, can be observed along the cylindrical axis in both maps. Because of the long $t_{\mathrm{a}}$ time adopted here, heavy component of oil is absent in all resolved $T_{1}$ profiles, while is still visible in $T_{2}$ profiles. Both projected $T_{1}$ and $T_{2}$ distributions are comparable with the rock experimental results shown in Fig. 6.7 (a) and (b). Furthermore, spatially resolved $T_{2}$ experiments were performed at confining temperatures of $55^{\circ} \mathrm{C}$ and $80^{\circ} \mathrm{C}$ in order 


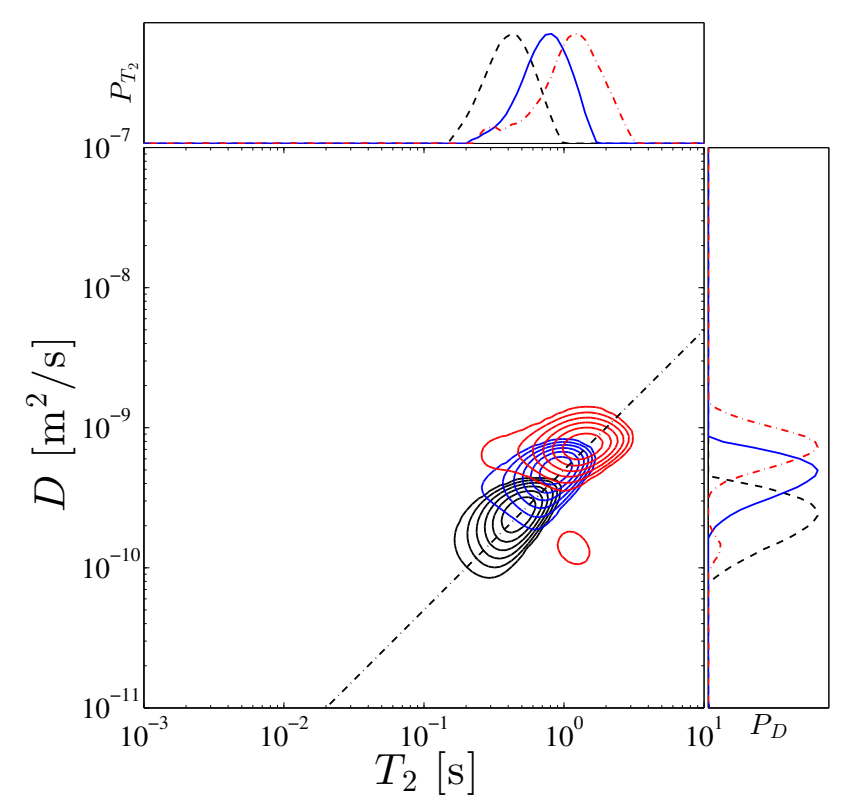

FIG. 6.8: Diffusion-relaxation time correlation $D-T_{2}$ distributions of the used oil-saturated rock core at 1000 psi. The slope dash line represents hydrocarbon correlation line of $D=5 \times 10^{-10} T_{2}$.

to investigate the oil temperature-dependent behaviour. The varying $T_{2}$ and spatial features as seen at $55^{\circ} \mathrm{C}$ and $80^{\circ} \mathrm{C}$ are not understood yet and remain subject to further research. Many reasons are probably ascribed to this phenomenon, one of which might be the partial drainage of the rock core. Nevertheless, projections onto the spatial and $T_{2}$ domains remain reasonable and confirm a shift of $T_{2}$ towards higher temperatures as expected.

In summary, the oil situated in the rock core becomes less viscous with increasing the confining temperature, which is revealed from $T_{1}$ and $T_{2}$ distributions as well as from the $D-T_{2}$ correlation distributions. The spatial $T_{2}$ profiles taken along the cylindrical core at different temperatures return distinct distributions and thus oil properties varying with temperature. 


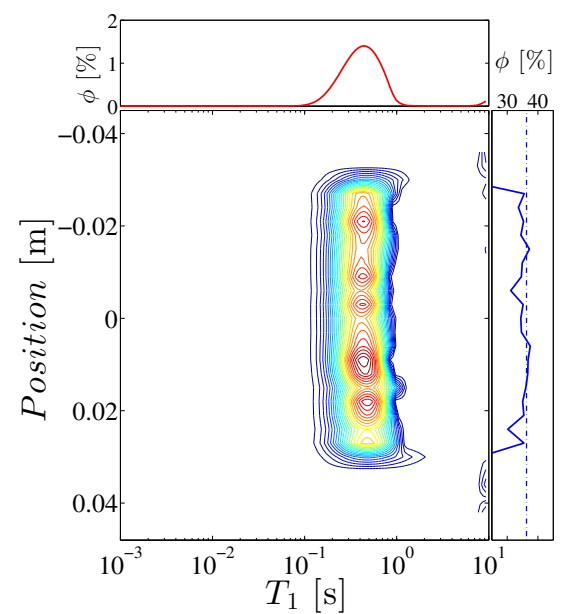

(a)

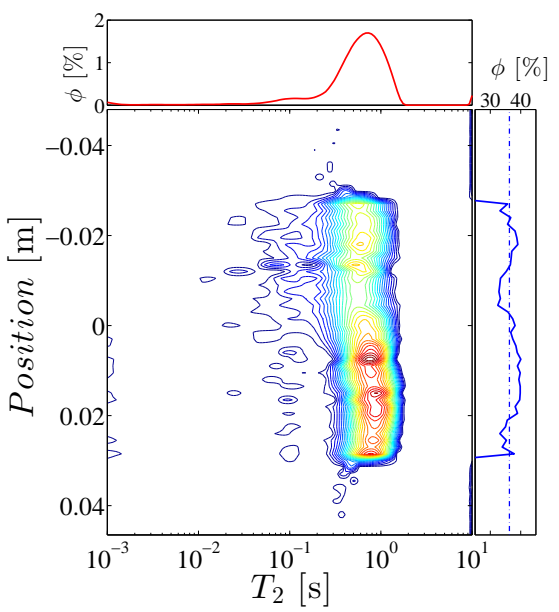

(c)

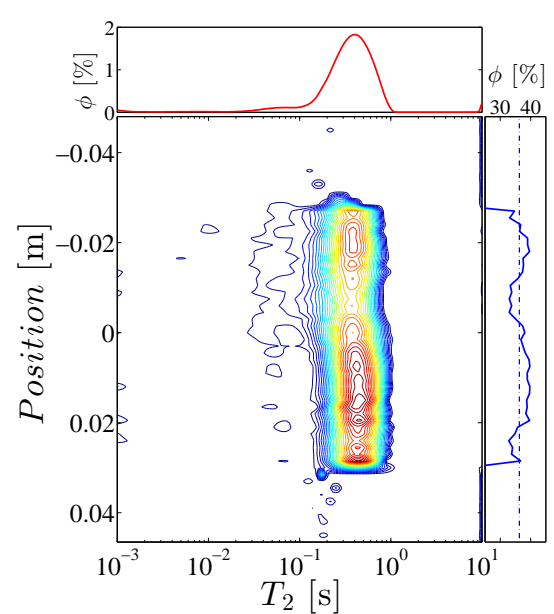

(b)

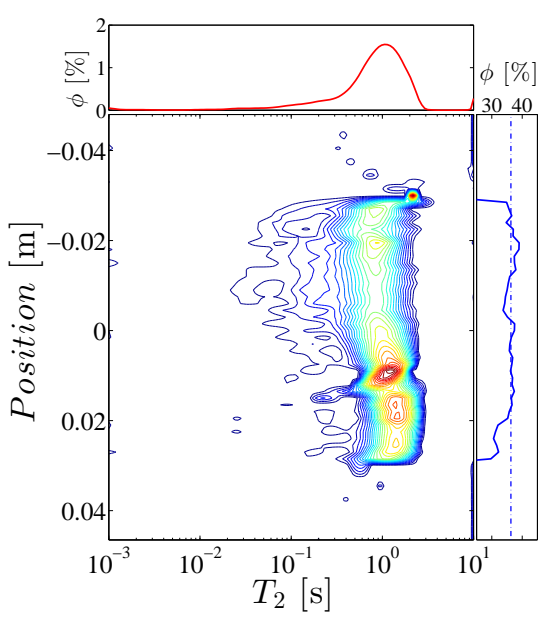

(d)

FIG. 6.9: (a) Spatially resolved $T_{1}$ profile at $25^{\circ} \mathrm{C}$ and $T_{2}$ profiles at (b) $25^{\circ} \mathrm{C}$, (c) $55^{\circ} \mathrm{C}$ and (d) $80^{\circ} \mathrm{C}$ at $1000 \mathrm{psi}$. The top panel presents the projected $T_{2}$ relaxation distribution and right panel gives the porosity profile. The blue dashed line in the right panel corresponds to the weighing porosity value measured in $25^{\circ} \mathrm{C}$. 


\subsubsection{In-situ water flooding measurement}

In this section, multi-phase fluid transport processes at different temperatures are observed by using the spatially resolved $D-T_{2}$ technique. The oil-saturated rock plug was firstly flooded by water at $25^{\circ} \mathrm{C}$ and 1000 psi. Subsequently, two further flooding steps at $55^{\circ} \mathrm{C}$ and $80^{\circ} \mathrm{C}$ were performed to investigate the temperature dependence during the flooding process. The spatially resolved $D-T_{2}$ profiles before, during and after water flooding at $25^{\circ} \mathrm{C}$ are compared and presented in Fig. 6.10. In order to visualize the existence of two different fluid phases, two reference planes indicating oil (left) and water (right) were added in to the figures as grey planes.

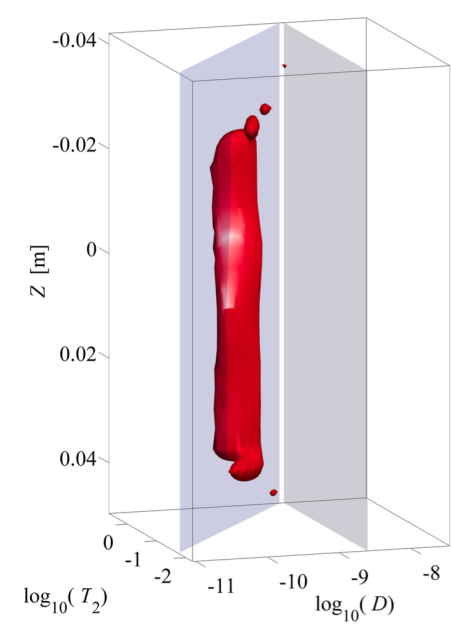

(a)

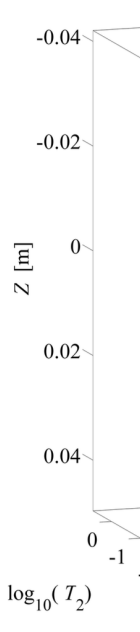

(b)

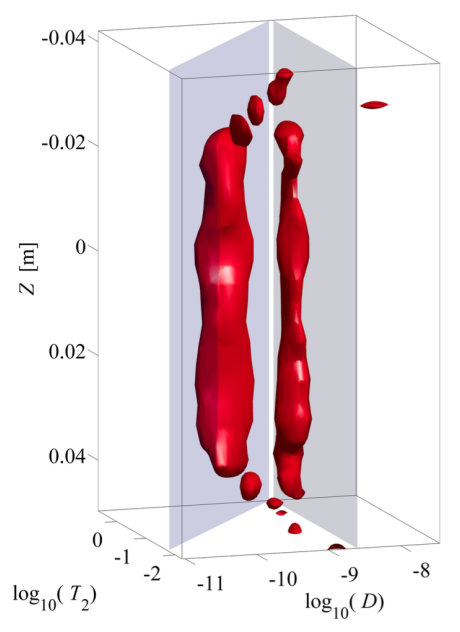

(c)

FIG. 6.10: Spatially resolved $D-T_{2}$ profile before water flooding (a), and after $0.1 \mathrm{PV}(\mathrm{b})$ and $1 \mathrm{PV}$ (c) water flooding at $25^{\circ} \mathrm{C}$. Two reference planes indicating hydrocarbon and water were plotted in order to identify the spatial distributions of oil and water signals.

The distribution function $F\left(z, D, T_{2}\right)$ before water flooding (shown in Fig. 6.10 (a)) mainly extends along the hydrocarbon plane indicating oil saturating from top to bottom in the rock plug. After flooding an amount of water corresponding to 0.1 Pore Volume (PV) through the rock, there appears a distinct distribution neighbouring the oil signal and laying along 
water reference plane (Fig. 6.10 (b)). This signal extends along the water plane after 1 PV water flooding (shown in Fig. 6.10 (c)). From the reference planes, it can be distinguished that the left column represents residual oil while the right one is the injected water. Therefore, it is feasible to extract the spatially resolved residual oil and water saturation from this distribution, and estimate oil recovery efficiency. The volume of flooding water was incremented up to 2.2 PV and two subsequent flooding experiments at $55^{\circ} \mathrm{C}$ and $80^{\circ} \mathrm{C}$ were performed with equal amount of water flooding.

The intensities of oil and water signals in the spatially resolved $D-T_{2}$ maps of each water flooding step can be readily integrated and plotted as relative water and oil saturation (shown in Fig. 6.11).

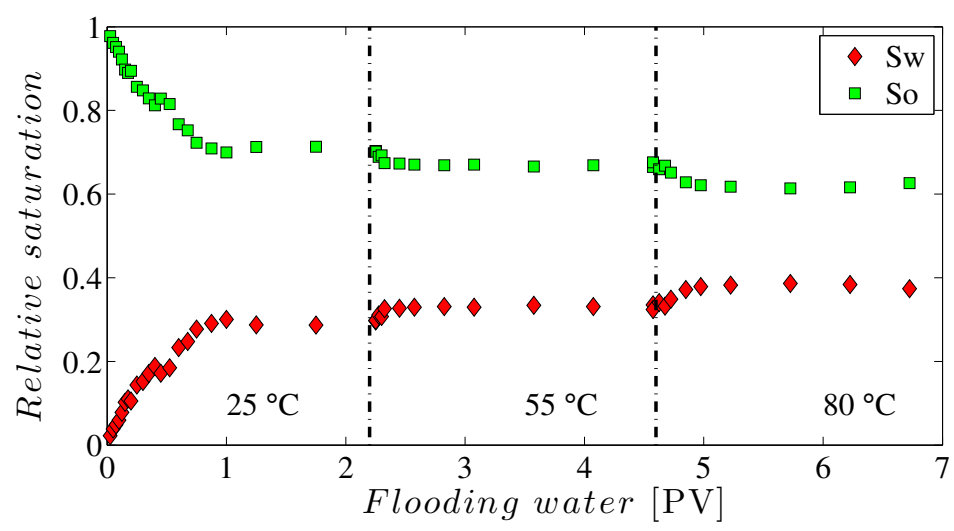

FIG. 6.11: Relative water (red diamond) and oil (green square) saturation estimated from the spatially resolved $D-T_{2}$ maps. The entire flooding procedure is performed under three confining temperatures $\left(25^{\circ} \mathrm{C}, 55^{\circ} \mathrm{C}\right.$ and $\left.80^{\circ} \mathrm{C}\right)$. The amount of the flooding water is normalized with the pore volume (PV) of the rock plug here. During the flooding in each temperature, the water volume is incremented to 2.2 PV.

For the water-flooding procedure at $25^{\circ} \mathrm{C}$, the relative oil saturation drops rapidly with increasing the flooding water volume, and reaches a plateau after 1 PV water injection. Further flooding shows no obvious oil yield in this rock plug. By increasing the rock temperature, the relative oil saturation continues to decrease because of the higher oil mobility, and quickly converges to a constant level again. The residual oil saturation is 
still above $50 \%$ even at rock temperatures up to $80^{\circ} \mathrm{C}$, which is a higher value as compared to common water-flooding-oil experiments (lower than $40 \%$ of residual oil saturation). This may due to the relatively high flow rate of the flooding water (approximately $0.25 \mathrm{~mL} / \mathrm{s}$ ) provided by the crank pump in this case. In order to investigate the multi-phase fluid interaction, all distributions at the end of flooding process under three temperatures were projected into the $D-T_{2}$ dimension and shown in Fig. 6.12. Regardless of longitudinal relaxation during the diffusion observation time, the ratio of water and oil varies gradually with increasing rock temperature, which can be observed from the projected diffusion coefficients in the right panel of each distribution.

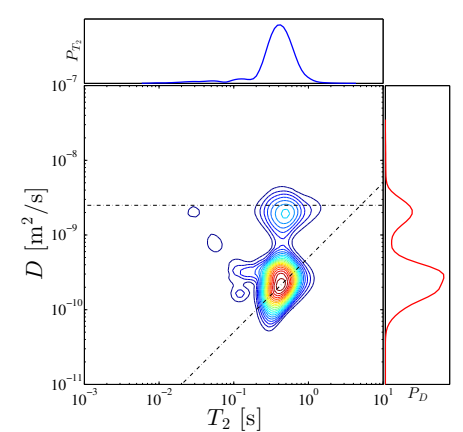

(a)

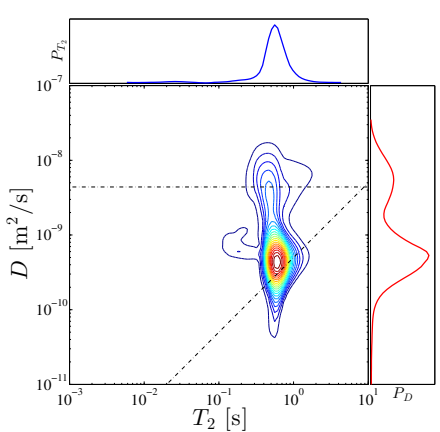

(b)

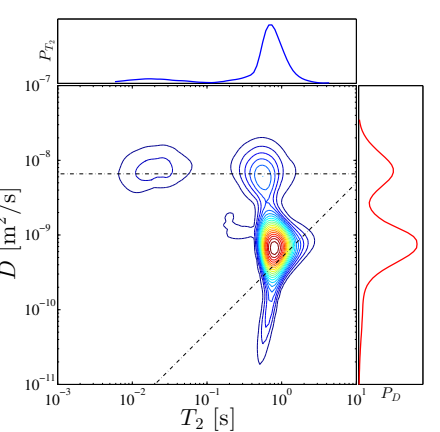

(c)

FIG. 6.12: Projected $D-T_{2}$ correlation distribution at (a) $25^{\circ} \mathrm{C}$, (b) $55^{\circ} \mathrm{C}$ and (c) $80^{\circ} \mathrm{C}$. The horizontal lines represent water diffusion coefficient at $25^{\circ} \mathrm{C}\left(=2.3 \times 10^{-9}\right.$ $\left.\mathrm{m}^{2} / \mathrm{s}\right)$, at $55^{\circ} \mathrm{C}\left(=4.4 \times 10^{-9} \mathrm{~m}^{2} / \mathrm{s}\right)$, and at $80^{\circ} \mathrm{C}\left(=6.6 \times 10^{-9} \mathrm{~m}^{2} / \mathrm{s}\right)$ [247]. The slope dash line represents hydrocarbon correlation line of $D=5 \times 10^{-10} T_{2}$. The top panel presents the projected relaxation distribution and right panel shows the projected diffusion coefficient distribution.

In the mean time, the 3D distributions at the end of flooding process under the three temperatures were also projected into the spatially-resolved $D$ profile and shown in Fig. 6.13. In these three maps, the relative saturation profiles of oil and water can be obtained and the gap between these two saturation profiles is narrower and narrower with increasing rock temperature, indicating the gradual output of oil from the flooded rock core. The 
results from Fig. 6.12 and Fig. 6.13 exhibit the same features as shown in the relative saturation data in Fig. 6.11, and imply a process of enhanced oil recovery by flooding the rock plug at higher temperatures. Further oil recovery strategy, such as gas, thermal or chemical polymer injection may be studied with the developed setup, potentially leading to a higher yield of hydrocarbons.

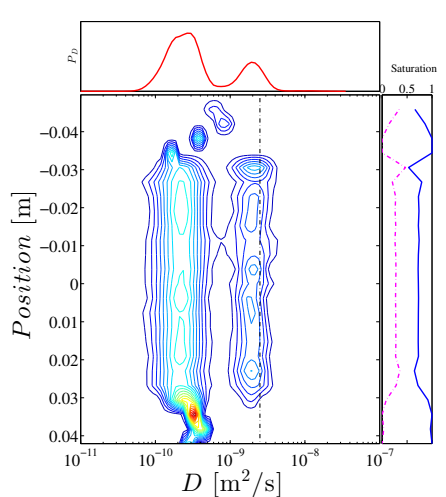

(a)

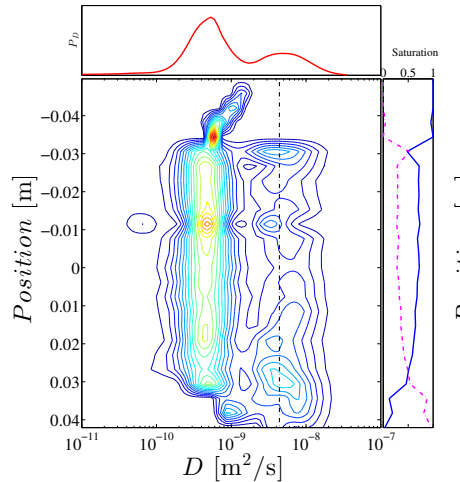

(b)

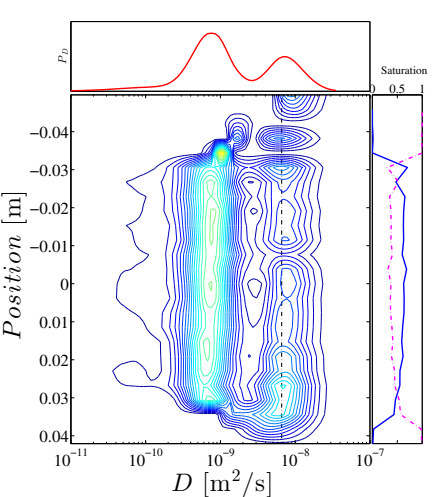

(c)

FIG. 6.13: Projected spatially resolved $D$ profiles at (a) $25^{\circ} \mathrm{C}$, (b) $55^{\circ} \mathrm{C}$ and (c) $80^{\circ} \mathrm{C}$. The vertical lines represent the water diffusion coefficient at $25^{\circ} \mathrm{C}\left(=2.3 \times 10^{-9}\right.$ $\left.\mathrm{m}^{2} / \mathrm{s}\right)$, at $55^{\circ} \mathrm{C}\left(=4.4 \times 10^{-9} \mathrm{~m}^{2} / \mathrm{s}\right)$, and at $80^{\circ} \mathrm{C}\left(=6.6 \times 10^{-9} \mathrm{~m}^{2} / \mathrm{s}\right)$. The top panel is the projected diffusion coefficient distribution while the right panel is the saturation profiles. The blue solid line is along the residual oil saturation and the pink dashed line indicates water saturation. 


\section{Chapter 7}

\section{Conclusions and discussions}

In this thesis, we proposed an integrated NMR toolbox within the developed techniques and implemented experimental set-up. These methodologies help expand our knowledge to three petrophysical aspects of fluid-bearing rocks, including pore length scales characterization, local permeability estimation and multi-phase fluids flooding evaluation.

Firstly, by extending the length of the phase encoding period $t_{\mathrm{e}}$, we successfully demonstrated that it is possible to use higher eigenmodes in the diffusion equation to determine the pore length scales of rock samples at frequencies as low as $2 \mathrm{MHz}$. The results we obtained at $2 \mathrm{MHz}$ were compared and confirmed by the measurements performed on a $64 \mathrm{MHz}$ system. This extension allows one to directly understand the pore sizes of rock cores using lower field, but cheaper NMR instruments, therefore, can largely broaden people's research on rock core analysis. Based on this knowledge, an eigenmode correlation experiment was proposed which coupled the high $T_{1}$ eigenmode with the ground $T_{2}$ mode at $2 \mathrm{MHz}$. By using this 2D approach, correlation maps of pore length scales $a$ and relaxation time $T_{2}$ have been obtained which also enable the direct extraction of surface relaxivity $\rho_{2}$. The experimental results of two rock cores with different lithology and their $\rho_{2}$ values have been confirmed independently through diffusion-relaxation correlation method. Both methods suggest 
larger $\rho_{2}$ values in sandstone as compared to the $\rho_{2}$ of limestone. Even though, it is still worth mentioning an intrinsic advantage of the eigenmode correlation method as compared to $D-T_{2}$ experiment in this context. Since the smaller transverse relaxation effects during the $1^{\text {st }}$ domain of this $2 \mathrm{D}$ experiment, it is possible to gain the details of the pores with comparably smaller scales and significant internal gradients using this eigenmode correlation method. So far, this approach has been adopted by other groups to extract surface relaxivity $\rho_{2}$ to calculate the relaxation/permeation length in porous materials [231] and to identify the surface interactions close to pore sinks when multi-phase fluids present in rock cores [248]. Beyond the above techniques, a further $2 \mathrm{D}$ variant in the eigenmode correlation framework was subsequently developed by coupling the high $T_{1}$ eigenmode with the ground $T_{1}$ mode at high-field ( $64 \mathrm{MHz}$ ). Besides the pore length $a-T_{1}$ correlation information as well as the surface relaxivity $\rho_{1}$ from it, the utilization of fast $T_{1}$ encoded in the $2^{\text {nd }}$ domain of this $2 \mathrm{D}$ approach provides a means for obtaining the reference signal without the need of a separate reference experiment (as necessary for the $T_{1}-T_{2}$ eigenmode correlation method). Moreover, the theory of $T_{1}-T_{1}$ eigenmode correlation method implies that there would be no negative peaks/distributions on 2D correlation maps because of the orthogonality of eigenfunctions from two successive domains. This will conveniently lead the dataset processing to be immune to the non-negative restraint during ILT analysis as discussed in Sec. 2.5.2. Since these two proposed 2D techniques offer alternative ways of determining surface relaxivities, it enables one to obtain pore geometry information from simple 1D NMR relaxation measurements after re-calibration of relaxivities to pore length scales.

In the next chapter, we moved forward to explore the permeability of rock cores by utilizing a specialized spatially-resolved $T_{1}$ profile technique based on rapid $T_{1}$ encoding method. The microscopic (pore length related) and macroscopic (heterogeneity) information of rock cores can be simultaneously obtained from the spatially-resolved $T_{1}$ maps. Since $T_{1}$ distribution 
indicates the pore length and pore structure in porous materials, a further factor $P^{i, i+1}$ characterizing the local connectedness between neighboring resolved slices was determined by calculating the matching degree of their $T_{1}$ distributions. Therefore, the permeability value of two adjoining resolved rock slices can be easily evaluated using the local porosity and the relaxation distributions from $T_{1}$ profile experimental results, optionally considering the connectedness factor $P$. The estimation of the local permeability can be consecutively executed along the cylindrical axis of rock plug and yield therefore its permeability profile. By performing the designed pulse sequence and the proposed model on two types of rock cores, we found that the permeability profiles reveal the variation of permeable property and associated spatial heterogeneity of the sample under studied. Moreover, we can conclude that the results considering the connectedness factor between adjacent slices along the flowing direction improves the evaluation of the local permeability when comparing the results to the measured brine permeability values. During the implementation of this spatially-resolved $T_{1}$ technique at low-field NMR context, we may notice that a compromise of experimental time and efficiency should be always taken into account as compared to its application at high-field, especially when adopting the tip angle $\alpha$ in the pulse sequence. Although utilizing $T_{1}$ imaging maps in this chapter, we believe that the idea of employing the defined local connectedness factor $P$ can be extended to any other kinds of spatially-resolved relaxation profile cases, for instance $T_{2}$ profiles at different field strengths, during evaluating the spatial permeability of rock cores.

In the last part, we studied the multi-phase fluid flooding process in rock plugs with the implemented high pressure/temperature NMR overburden system. We started this chapter from the stationary measurements, which means that no flooding provided for the saturating fluids. The employed spatially resolved $T_{1}$ and $T_{2}$ relaxation techniques provide direct insight into the local properties of fluid situated in rock cores at reservoir-like 
conditions. Besides that, spatially resolved $D-T_{2}$ method yields substantial information such as wetting status, residual oil/water saturation profiles, and their dependence on temperature. Regarding the flooding experiments, the hardware set-up and the implemented NMR methods provide the base for the investigations of displacement front and residual oil saturation in the context of enhancement oil recovery. By incrementally increasing the amount of flooding water, the experimental results suggested a process of enhanced oil recovery by flooding the rock plug at higher temperatures.

Two further streams could be possibly carried out from this thesis. Firstly, it would be applicable to extend the strategy of permeability estimation within the proposed connectedness factor $P$ in Chapter 5 to 2D or even 3D cases relying on the combination of high-dimensional MR imaging techniques with relaxation measurements. If considering the directional dependence of the proposed connectedness factor, this extension will allow one to characterize the variation of permeability properties in any spatial path. More interestingly, the emerging upscaling techniques are more suitable on higher-dimensional NMR results, thus the renormalized permeability values can be utilized to evaluate the permeability anisotropy and flow dispersion along different fluid transport directions in rock samples $[238,239]$. Another potential work is to upgrade the entire hardware system to supply more reasonable flooding flow rates with proper pumps, therefore quantifying the fluid transport and recovery in a more realistic way in Chapter 6. Based on this, some appropriate fast imaging methods, as well as the compressed sensing technique, which has been already used in the MRI rock core analysis [249, 250], might be introduced in order to accelerate the data acquisition during spatially resolved $D-T_{2}$ technique. 


\section{Conference Attendance}

1. H. Liu, M. N. d'Eurydice, P. Galvosas, The $12^{\text {th }}$ International Conference on Magnetic Resonance Microscopy, oral presentation, London, 2013.

2. H. Liu, M. N. d'Eurydice, P. Galvosas, The $5^{\text {th }}$ Asia-Pacific NMR symposium \& The $9^{\text {th }}$ Australian and New Zealand Society for Magnetic Resonance, poster presentation, Brisbane, 2013.

3. H. Liu, M. N. d'Eurydice, P. Galvosas, The $12^{\text {th }}$ Magnetic Resonance in Porous Media, poster presentation, Wellington, 2014.

4. H. Liu, M. N. d'Eurydice, P. Galvosas, European Congress on Magnetic Resonance, poster presentation, Zürich, 2014.

5. H. Liu, M. N. d'Eurydice, S. Obruchkov, P. Galvosas, The $7^{\text {th }}$ Advanced Materials \& Nanotechnology, oral presentation, Nelson, 2015.

6. H. Liu, M. N. d'Eurydice, S. Obruchkov, P. Galvosas, The $13^{\text {th }}$ International Conference on Magnetic Resonance Microscopy, oral presentation, Münich, 2015.

7. H. Liu, M. Hunter, S. Obruchkov, E. McCarney, M. Robison, R. Dykstra, P. Galvosas, The $29^{\text {th }}$ International Symposium of the Society of Core Analysts, oral presentation, St. John's, 2015. 


\section{Bibliography}

[1] D. Tiab and E. C. Donaldson, Petrophysics: theory and practice of measuring reservoir rock and fluid transport properties. Gulf professional publishing, 2011.

[2] A. Y. Dandekar, Petroleum reservoir rock and fluid properties. CRC press, 2013.

[3] P. Wong, T. Lucatorto, and M. D. Graef, Methods of the Physics of Porous Media. Academic Press, 1999.

[4] D. Ellis and J. Singer, Well logging for earth scientists. Springer, 2007.

[5] K. Dunn, D. Bergman, and G. LaTorraca, Nuclear magnetic resonance: Petrophysical and logging applications. Elsevier, 2002.

[6] J. Mitchell, T. Chandrasekera, D. Holland, L. Gladden, and E. Fordham, "Magnetic resonance imaging in laboratory petrophysical core analysis," Physics Reports, 2013.

[7] J. Mitchell, L. F. Gladden, T. C. Chandrasekera, and E. J. Fordham, "Low-field permanent magnets for industrial process and quality control," Progress in Nuclear Magnetic Resonance Spectroscopy, vol. 76, pp. 1-60, Jan. 2014.

[8] J. Mitchell, "Magnetic resonance core analysis at $0.3 \mathrm{~T}$," International Symposium of the Society of Core Analysis, Sept. 2014-10. 
[9] M. D. Hürlimann and L. Venkataramanan, “Quantitative measurement of two-dimensional distribution functions of diffusion and relaxation in grossly inhomogeneous fields," Journal of Magnetic Resonance, vol. 157, no. 1, pp. 31-42, 2002.

[10] B. Sun, M. Olson, and J. Baranowski, "Direct Fluid Typing and Quantification of Orinoco Belt Heavy Oil Reservoirs Using 2D NMR Logs," 47th SPWLA Annual Logging Symposium, 2006.

[11] M. D. Hürlimann and M. Flaum, "Diffusion-relaxation distribution functions of sedimentary rocks in different saturation states," Magnetic resonance imaging, vol. 21, pp. 305-310, 2003.

[12] K.-h. Kim, S. Chen, F. Qin, and A. Watson, "Use of NMR Imaging for Determining Fluid Saturation Distributions During Multiphase Displacement in Porous Media," International Symposium of the Society of Core Analysis, 1992-9219.

[13] R. L. Kleinberg, C. Straley, W. E. Kenyon, R. Akkurt, and S. Farooqui, “Nuclear Magnetic Resonance of Rocks: T1 vs T2," Society of Petroleum Engineers, 1993.

[14] R. Kleinberg, S. Farooqui, and M. Horsfield, “T1/T2 Ratio and Frequency Dependence of NMR Relaxation in Porous Sedimentary Rocks," Journal of Colloid and Interface Science, vol. 158, pp. 195-198, June 1993.

[15] L. Latour, P. Mitra, R. Kleinberg, and C. Sotak, “Time-Dependent Diffusion Coefficient of Fluids in Porous Media as a Probe of Surfaceto-Volume Ratio," 1993.

[16] G. Coates, L. Xiao, and M. Prammer, NMR logging: principles and applications. Halliburton Energy Services, 1999. 
[17] Y. Song and S. Ryu, "Nuclear magnetic resonance method and apparatus for determining pore characteristics of rocks and other porous materials," US Patent 6,369,567, vol. 1, no. 12, 2002.

[18] C. Arns, M. Knackstedt, and N. Martys, "Cross-property correlations and permeability estimation in sandstone," Physical Review E, vol. 339, pp. 152-158, Aug. 2005.

[19] A. Timur, "Pulsed nuclear magnetic resonance studies of porosity, movable fluid permeability of sandstones," Journal of Petroleum Technology, vol. 21, pp. 775-786, 1969.

[20] J. R. Banavar and L. M. Schwartz, "Magnetic-Resonance as a Probe of Permeability in Porous Media," Physical Review Letters, vol. 58, no. 14, pp. 1411-1414, 1987.

[21] W. Kenyon, P. Day, C. Straley, and J. Willemsen, "A Three-Part Study of NMR Longitudinal Relaxation Properties of Water-Saturated Sandstones," SPE Formation Evaluation, vol. 3, no. 3, 1988.

[22] W. Kenyon, "Petrophysical principles of applications of NMR logging," Log Analyst, 1997.

[23] L. Utracki, "Temperature and pressure dependence of liquid viscosity," The Canadian Journal of Chemical Engineering, vol. 61, no. 5, pp. $753-758,1983$.

[24] M. D. Hürlimann, "The diffusion-spin relaxation time distribution function as an experimental probe to characterize fluid mixtures in porous media," The Journal of chemical physics, vol. 117, no. 22, pp. 10223-10232, 2002.

[25] M. D. Hürlimann, M. Flaum, L. Venkataramanan, C. Flaum, R. Freedman, and G. J. Hirasaki, “Diffusion-relaxation distribution functions 
of sedimentary rocks in different saturation states," Magnetic Resonance Imaging, vol. 21, pp. 305-310, Apr. 2003.

[26] M. N. d'Eurydice and P. Galvosas, "Measuring diffusion-relaxation correlation maps using non-uniform field gradients of single-sided NMR devices," Journal of Magnetic Resonance, vol. 248, pp. 137-145, 2014.

[27] A. Abragam, The principles of nuclear magnetism. Oxford University Press, 1961.

[28] C. P. Slichter, "Principles of magnetic resonance, with examples from solid state physics," 1963.

[29] F. Bloch, "Nuclear induction," Physical review, vol. 70, pp. 460-474, 1946.

[30] E. Hahn, "Spin echoes," Physical Review, vol. 80, no. 4, pp. 580-594, 1950.

[31] R. Kimmich, NMR: tomography, diffusometry, relaxometry. SpringerVerlag, 1997.

[32] T. Chandrasekera, J. Mitchell, E. Fordham, L. Gladden, and M. Johns, "Rapid encoding of $T_{1}$ with spectral resolution in n-dimensional relaxation correlations," Journal of Magnetic Resonance, vol. 194, no. 1, pp. 156-161, 2008.

[33] H. Y. Carr and E. M. Purcell, "Effects of diffusion on free precession in nuclear magnetic resonance experiments," Physical Review, vol. 94, no. 3, pp. 630-638, 1954.

[34] S. Meiboom and D. Gill, "Modified Spin-Echo Method for Measuring Nuclear Relaxation Times," Review of Scientific Instruments, vol. 29, no. 8 , p. $688,1958$. 
[35] F. Casanova, J. Perlo, and B. Blümich, Single-sided NMR. Springer, 2011.

[36] J. Crank, The mathematics of diffusion, vol. 2. Clarendon press Oxford, 1975.

[37] P. Grathwohl, Diffusion in natural porous media. Kluwer, 2002.

[38] P. T. Callaghan, Principles of nuclear magnetic resonance microscopy, vol. 3. Clarendon Press Oxford, 1991.

[39] E. O. Stejskal, "Use of Spin Echoes in a Pulsed Magnetic-Field Gradient to Study Anisotropic, Restricted Diffusion and Flow," The Journal of Chemical Physics, vol. 43, no. 10, p. 3597, 1965.

[40] J. Tanner, "Use of the stimulated echo in NMR diffusion studies," The Journal of Chemical Physics, vol. 52, no. 5, pp. 2523-2526, 1970.

[41] Kittler, W.C. and Galvosas, P. and Hunter, M.W., "Parallel Acquisition of q-Space Using Second Order Magnetic Fields for Single-Shot Diffusion Measurements," Journal of Magnetic Resonance, Apr. 2014.

[42] M. D. Hürlimann, "Diffusion and relaxation effects in general stray field NMR experiments," Journal of magnetic resonance, vol. 148, pp. 367-78, Feb. 2001.

[43] Y.-Q. Song, "Categories of Coherence Pathways for the CPMG Sequence," Journal of Magnetic Resonance, vol. 157, pp. 82-91, July 2002.

[44] E. E. Sigmund, H. Cho, and Y.-Q. Song, "Multiple-modulationmultiple-echo magnetic resonance," Concepts in Magnetic Resonance Part A, vol. 30A, pp. 358-377, Nov. 2007.

[45] P. C. Lauterbur et al., "Image formation by induced local interactions: examples employing nuclear magnetic resonance," Nature, vol. 242, no. 5394, pp. 190-191, 1973. 
[46] P. Mansfield and P. K. Grannell, "NMR "diffraction" in solids?," Journal of Physics C: solid state physics, vol. 6, no. 22, p. L422, 1973.

[47] P. Morris, NMR imaging in biology and medicine. Clarendon Press Oxford, 1986.

[48] B. Blümich, NMR Imaging of Materials. Oxford University Press, 2000.

[49] P. Callaghan, Translational dynamics and magnetic resonance: principles of pulsed gradient spin echo NMR. Oxford University Press, 2011.

[50] Q. Chen, F. R. Rack, and B. J. Balcom, “Quantitative magnetic resonance imaging methods for core analysis," Geological Society, London, Special Publications, vol. 267, no. 1, pp. 193-207, 2006.

[51] E. Haacke, R. Brown, M. Thompson, R. Venkatesan, M. Bernstein, K. King, and X. Zhou, "Magnetic resonance imaging-physical principles and sequence design," 1999.

[52] R. R. Ernst, G. Bodenhausen, A. Wokaun, et al., Principles of nuclear magnetic resonance in one and two dimensions. Clarendon Press Oxford, 1987.

[53] A. A. Khrapitchev, S. Han, S. Stapf, and B. Blümich, "Spectrally resolved velocity exchange spectroscopy of two-phase flow," Journal of Magnetic Resonance, vol. 159, no. 1, pp. 36-45, 2002.

[54] S. Han, S. Stapf, and B. Blümich, "Fluid transport and filtration in a hemodialyzer module by 2D PFG NMR," 2001.

[55] B. Blümich, P. T. Callaghan, R. A. Damion, S. Han, A. A. Khrapitchev, K. J. Packer, and S. Stapf, "Two-dimensional NMR of velocity exchange: VEXSY and SERPENT.," Journal of magnetic resonance, vol. 152, pp. 162-167, Sept. 2001. 
[56] S. Stapf, K. Packer, S. Békri, and P. Adler, "Two-dimensional nuclear magnetic resonance measurements and numerical simulations of fluid transport in porous rocks," Physics of Fluids, vol. 12, no. 3, pp. 566-580, 2000.

[57] S. Han, S. Stapf, and B. Blumich, "Two-dimensional PFG NMR for encoding correlations of position, velocity, and acceleration in fluid transport," Journal of magnetic resonance, vol. 146, pp. 169-80, Sept. 2000.

[58] L. M. Burcaw and P. T. Callaghan, "Observation of molecular migration in porous media using 2D exchange spectroscopy in the inhomogeneous magnetic field," Journal of Magnetic Resonance, vol. 198, pp. 167-173, June 2009.

[59] L. M. Burcaw, M. W. Hunter, and P. T. Callaghan, "Propagatorresolved 2D exchange in porous media in the inhomogeneous magnetic field," Journal of Magnetic Resonance, vol. 205, pp. 209-215, Aug. 2010.

[60] L. M. Burcaw and P. T. Callaghan, "Correlation of the inhomogeneous field as a measure of sample heterogeneity," Journal of Magnetic Resonance, vol. 216, pp. 144-151, Mar. 2012.

[61] F. B. Laun, T. A. Kuder, W. Semmler, and B. Stieltjes, “Determination of the Defining Boundary in Nuclear Magnetic Resonance Diffusion Experiments," Physical Review Letters, vol. 107, p. 048102, July 2011.

[62] S. Hertel, M. Hunter, and P. Galvosas, "Magnetic resonance pore imaging, a tool for porous media research," Physical Review E, vol. 87, p. 030802, Mar. 2013.

[63] Y.-Q. Song, L. Venkataramanan, M. D. Hürlimann, M. Flaum, P. Frulla, and C. Straley, " $T_{1}-T_{2}$ correlation spectra obtained using a fast 
two-dimensional Laplace inversion," Journal of Magnetic Resonance, vol. 154, no. 2, pp. 261-268, 2002.

[64] Y. Qiao, P. Galvosas, and P. T. Callaghan, "Diffusion correlation NMR spectroscopic study of anisotropic diffusion of water in plant tissues," Biophysical journal, vol. 89, pp. 2899-905, Oct. 2005.

[65] P. McDonald, J.-P. Korb, J. Mitchell, and L. Monteilhet, "Surface relaxation and chemical exchange in hydrating cement pastes: a twodimensional NMR relaxation study," Physical Review E, vol. 72, no. 1, p. 011409, 2005.

[66] P. T. Callaghan, C. Arns, and P. Galvosas, "Recent Fourier and Laplace perspectives for multidimensional NMR in porous media," Magnetic Resonance Imaging, vol. 25, no. 4, pp. 441-444, 2007.

[67] P. Galvosas, Y. Qiao, S. Monika, and P. T. Callaghan, "On the use of 2D correlation and exchange NMR spectroscopy in organic porous materials," Magnetic Resonance Imaging, vol. 25, pp. 497-500, May 2007.

[68] M. Gratz, M. Wehring, P. Galvosas, and F. Stallmach, "Multidimensional NMR diffusion studies in microporous materials," Microporous and Mesoporous Materials, vol. 125, pp. 30-34, Oct. 2009.

[69] K. E. Washburn and P. T. Callaghan, "Tracking pore to pore exchange using relaxation exchange spectroscopy," Physical Review Letters, vol. 97, p. 175502, Oct. 2006.

[70] Y. Qiao, P. Galvosas, T. Adalsteinsson, M. Schönhoff, and P. T. Callaghan, "Diffusion exchange NMR spectroscopic study of dextran exchange through polyelectrolyte multilayer capsules," Journal of Chemical Physics, vol. 122, p. 214912, June 2005. 
[71] K. F. Morris and C. S. Johnson, "Diffusion-ordered 2-dimensional nuclear-magnetic-resonance spectroscopy," Journal of the American Chemical Society, vol. 114, pp. 3139-3141, 1992.

[72] J.-F. Kuntz, P. Palmas, V. Level, and D. Canet, "Restricted diffusion and exchange of water in porous media: average structure determination and size distribution resolved from the effect of local field gradients on the proton NMR spectrum.," Journal of magnetic resonance, vol. 191, pp. 239-47, Apr. 2008.

[73] M. M. Britton, R. G. Graham, and K. J. Packer, "NMR relaxation and pulsed field gradient study of alginate bead porous media.," Journal of magnetic resonance, vol. 169, pp. 203-214, Aug. 2004.

[74] J. Mitchell, D. A. Graf von der Schulenburg, D. J. Holland, E. Fordham, M. L. Johns, and L. Gladden, "Determining NMR flow propagator moments in porous rocks without the influence of relaxation.," Journal of magnetic resonance, vol. 193, pp. 218-25, Aug. 2008.

[75] K. E. Washburn, C. H. Arns, and P. T. Callaghan, "Pore characterization through propagator-resolved transverse relaxation exchange," Physical Review E, vol. 77, p. 051203, May 2008.

[76] B. Sun, K.-j. Dunn, G. A. Latorraca, and D. M. Wilson, “NMR imaging with diffusion and relaxation," International Symposium of the Society of Core Analysis, Sept. 2003-24.

[77] O. V. Petrov and B. J. Balcom, "Two-dimensional $T_{2}$ distribution mapping in porous solids with phase encode MRI," Journal of Magnetic Resonance, vol. 212, pp. 102-108, Sept. 2011.

[78] D. Xiao and B. J. Balcom, “Two-dimensional $T_{2}$ distribution mapping in rock core plugs with optimal k-space sampling," Journal of Magnetic Resonance, vol. 220, pp. 70-78, July 2012. 
[79] M. Rauschhuber and G. Hirasaki, "Determination of saturation profiles via low-field NMR imaging," International Symposium of the Society of Core Analysis, Sept. 2009-09.

[80] Y. Zhang and B. Blümich, "Spatially resolved $D-T_{2}$ correlation NMR of porous media.," Journal of magnetic resonance, vol. 242C, pp. 41-48, Feb. 2014.

[81] N. Shemesh, C.-F. Westin, and Y. Cohen, "Magnetic Resonance Imaging by Synergistic Diffusion-Diffraction Patterns," Physical Review Letters, vol. 108, p. 058103, Jan. 2012.

[82] H. Liu, M. Nogueira d'Eurydice, S. Obruchkov, and P. Galvosas, "Determining pore length scales and pore surface relaxivity of rock cores by internal magnetic fields modulation at 2MHz NMR.," Journal of magnetic resonance, vol. 246C, pp. 110-118, 2014.

[83] H. Liu, M. Nogueira d'Eurydice, S. Obruchkov, and P. Galvosas p. In preparation, 2015.

[84] C. H. Arns, K. E. Washburn, P. T. Callaghan, et al., "Multidimensional NMR Inverse Laplace Spectroscopy In Petrophysics1," Petrophysics, vol. 48, no. 05, 2007.

[85] K. Washburn, C. Eccles, and P. T. Callaghan, "The dependence on magnetic field strength of correlated internal gradient relaxation time distributions in heterogeneous materials," Journal of Magnetic Resonance, vol. 194, pp. 33-40, Sept. 2008.

[86] L. Xiao, H. Liu, F. Deng, Z. Zhang, T. An, F. Zong, V. Anferov, and S. Anferova, "Probing internal gradients dependence in sandstones with multi-dimensional NMR," Microporous and Mesoporous Materials, vol. 178, pp. 90-93, Sept. 2013.

[87] B. Sun and K. Dunn, "Core analysis with two dimensional NMR," International Symposium of the Society of Core Analysis, 2002. 
[88] http: / /www.healthcare.siemens.com/ magnetic-resonance-imaging

[89] http://www.magritek.com

[90] https://www.bruker.com/products/mr.html

[91] C. L. Lawson and R. J. Hanson, Solving least squares problems, vol. 161. SIAM, 1974.

[92] N. J. Heaton, "Multi-measurement NMR analysis based on maximum entropy," US Patent 6,960,913, 2005.

[93] A. N. Tikhonov and V. Y. Arsenin, Solutions of ill-posed problems. V.H. Winson Sons, 1977.

[94] J. P. Butler, J. A. Reeds, and S. V. Dawson, "Estimating Solutions of First Kind Integral Equations with Nonnegative Constraints and Optimal Smoothing," 1981.

[95] L. Venkataramanan, Y.-Q. Song, and M. D. Hürlimann, "Solving Fredholm Integrals of the First Kind With Tensor Product Structure in 2 and 2.5 Dimensions," IEEE Transactions on Signal Processing, vol. 50, no. 5, pp. 1017-1026, 2002.

[96] E. Chouzenoux, S. Moussaoui, J. Idier, and F. Mariette, “Efficient Maximum Entropy Reconstruction of Nuclear Magnetic Resonance $T_{1}-T_{2}$ Spectra," IEEE Transactions on Signal Processing, vol. 58, pp. 6040 6051, Dec. 2010.

[97] J. Mitchell, T. C. Chandrasekera, and L. Gladden, "Numerical estimation of relaxation and diffusion distributions in two dimensions.," Progress in nuclear magnetic resonance spectroscopy, vol. 62, pp. 34-50, Apr. 2012. 
[98] Z. F. Zhang, L. Z. Xiao, H. B. Liu, F. Deng, X. Li, T. L. An, V. Anferov, and S. Anferova, "A Fast Three-Dimensional Protocol for Low-Field Laplace NMR in Porous Media," Applied Magnetic Resonance, vol. 44, pp. 849-857, May 2013.

[99] B. Sun and K. J. Dunn, "A global inversion method for multidimensional NMR logging," Journal of Magnetic Resonance, vol. 172, pp. 152-160, Jan. 2005.

[100] B. Sun and K. J. Dunn, "Methods and limitations of NMR data inversion for fluid typing," Journal of Magnetic Resonance, vol. 169, pp. 118128, July 2004.

[101] Y.-Q. Song, L. Venkataramanan, and L. Burcaw, “Determining the resolution of Laplace inversion spectrum.," The Journal of chemical physics, vol. 122, p. 104104, Mar. 2005.

[102] R. L. Parker and Y.-Q. Song, "Assigning uncertainties in the inversion of NMR relaxation data.," Journal of magnetic resonance, vol. 174, pp. 314-24, June 2005.

[103] Y.-Q. Song, "Resolution and uncertainty of Laplace inversion spectrum.," Magnetic resonance imaging, vol. 25, pp. 445-8, May 2007.

[104] M. Prange and Y.-Q. Song, "Quantifying uncertainty in NMR $T_{2}$ spectra using Monte Carlo inversion.," Journal of magnetic resonance, vol. 196, pp. 54-60, Jan. 2009.

[105] M. Prange and Y.-Q. Song, "Understanding NMR $T_{2}$ spectral uncertainty.," Journal of magnetic resonance, vol. 204, pp. 118-123, May 2010 .

[106] L. Venkataramanan and F. Gruber, "Mellin transform of CPMG data," Journal of Magnetic Resonance, vol. 206, pp. 20-31, Sept. 2010. 
[107] H. Zhu, H. P. Huinink, P. C. M. M. Magusin, O. C. G. Adan, and K. Kopinga, " $T_{2}$ distribution spectra obtained by continuum fitting method using a mixed Gaussian and Exponential kernel function.," Journal of magnetic resonance, pp. 109-114, Oct. 2013.

[108] F. K. Gruber, L. Venkataramanan, T. M. Habashy, and D. E. Freed, "Estimation of petrophysical and fluid properties using integral transforms in nuclear magnetic resonance.," Journal of magnetic resonance, vol. 228, pp. 104-15, Mar. 2013.

[109] F. K. Gruber, L. Venkataramanan, T. M. Habashy, P. M. Singer, and D. E. Freed, "A more accurate estimate of $T_{2}$ distribution from direct analysis of NMR measurements.," Journal of magnetic resonance (San Diego, Calif. : 1997), vol. 228, pp. 95-103, Mar. 2013.

[110] Z.-M. Jiang and W.-M. Wang, "A Method of Choosing the Optimal Number of Singular Values in the Inverse Laplace Transform for the Two-Dimensional NMR Distribution Function," Chinese Physics Letters, vol. 30, p. 010201, Jan. 2013.

[111] P. Berman, O. Levi, Y. Parmet, M. Saunders, and Z. Wiesman, "Laplace inversion of low-resolution NMR relaxometry data using sparse representation methods," Concepts in Magnetic Resonance Part A: Bridging Education and Research, vol. 42, no. 3, pp. 72-88, 2013.

[112] E. Tø nning and D. Polders, "A novel improved method for analysis of 2D diffusion-relaxation data-2D PARAFAC-Laplace decomposition," Journal of Magnetic Resonance, vol. 188, pp. 10-23, Sept. 2007.

[113] K. E. Washburn, E. Anderssen, S. J. Vogt, J. D. Seymour, J. E. Birdwell, C. M. Kirkland, and S. L. Codd, "Simultaneous Gaussian and exponential inversion for improved analysis of shales by NMR relaxometry," Journal of Magnetic Resonance, vol. 250, pp. 7-16, 2015. 
[114] H. Vinegar, P. Tutunjian, W. Edelstein, P. Roemer, et al., "Wholecore analysis by ${ }^{13} \mathrm{C}$ NMR," Society of Petroleum Engineers Formation Evaluation, vol. 6, no. 02, pp. 183-189, 1991.

[115] L. Xiao, Y. Du, and C. Ye, " ${ }^{13}$ C Longitudinal Relaxation of Fluids Saturated in Oil Reservoir Cores by MAS NMR," Journal of colloid and interface science, vol. 164, no. 2, pp. 495-497, 1994.

[116] L. Rijniers, P. Magusin, H. Huinink, L. Pel, and K. Kopinga, "Sodium NMR relaxation in porous materials," Journal of Magnetic Resonance, vol. 167, no. 1, pp. 25-30, 2004.

[117] K. E. Washburn and G. Madelin, "Imaging of multiphase fluid saturation within a porous material via sodium NMR," Journal of Magnetic Resonance, vol. 202, no. 1, pp. 122-126, 2010.

[118] S. Sarkar, J. Dechter, and R. Komoroski, "Multinuclear NMR imaging of fluid phases in Berea sandstone," Journal of Magnetic Resonance, vol. 102, no. 3, pp. 314-317, 1993.

[119] R. W. Mair, G. Wong, D. Hoffmann, M. D. Hürlimann, S. Patz, L. M. Schwartz, and R. L. Walsworth, "Probing porous media with gas diffusion NMR," Physical review letters, vol. 83, no. 16, p. 3324, 1999.

[120] R. Wang, R. Mair, M. Rosen, D. Cory, and R. Walsworth, "Simultaneous measurement of rock permeability and effective porosity using laser-polarized noble gas NMR," Physical Review E, vol. 70, p. 026312, Aug. 2004.

[121] J. Granwehr, E. Harel, S. Han, S. Garcia, a. Pines, P. Sen, and Y.-Q. Song, "Time-of-Flight Flow Imaging Using NMR Remote Detection," Physical Review Letters, vol. 95, p. 075503, Aug. 2005.

[122] http://chem.ch.huji.ac.il/nmr/techniques/1d/multi. html 
[123] W. Rotwell and H. Vinegar, "Petrophysical Application of NMR Imaging," Applied Optics, vol. 24, pp. 3969-3972, 1985.

[124] P. Osment, K. Packer, M. Taylor, J. Attard, T. Carpenter, L. Hall, N. Herrod, and S. Doran, "NMR imaging of fluids in porous solids," Philosophical Transactions: Physcial Sciences and Engineering, vol. 333, no. 1632, pp. 441-452, 1990.

[125] A. Peters, P. Robyr, R. Bowtell, and P. Mansfield, “Echo-planar microscopy of porous rocks," Magnetic Resonance Imaging, vol. 14, no. 7, pp. 875-877, 1996.

[126] B. J. Balcom, R. P. MacGregor, S. D. Beyea, D. P. Green, R. L. Armstrong, and T. W. Bremner, "Single-Point Ramped Imaging with $T_{1}$ Enhancement (SPRITE)," Journal of Magnetic Resonance, vol. 123, no. 1, pp. 131-134, 1996.

[127] B. Issa, M. a. Al-Mugheiry, and P. Mansfield, "Transient flow through porous rocks studied by PEPI," Magnetic Resonance Imaging, vol. 14, no. 7-8, pp. 933-935, 1996.

[128] R. Kleinberg, a. Sezginer, D. Griffin, and M. Fukuhara, "Novel NMR apparatus for investigating an external sample," Journal of Magnetic Resonance, vol. 97, pp. 466-485, May 1992.

[129] D. Allen, C. Flaum, and T. Ramakrishnan, "Trends in NMR logging," Oilfield Review, pp. 2-19, 2000.

[130] R. L. Kleinberg, “Well logging overview," Concepts in Magnetic Resonance, vol. 13, no. 6, pp. 342-343, 2001.

[131] B. Blümich, S. Haber-Pohlmeier, and W. Zia, Compact NMR. Walter de Gruyter GmbH, 2014.

[132] M. Prammer and R. Akkurt, "A new direction in wireline and LWD NMR," SPWLA 43rd Annual Logging Symposium, 2002. 
[133] S. Chen, L. Li, G. Zhang, and J. Chen, "Magnetic resonance for downhole complex-lithology earth formation evaluation," New Journal of Physics, vol. 13, p. 085015, Aug. 2011.

[134] http://www.oxford-instruments.com/ industries-and-applications/energy-environment/ core-analysis

[135] http://www.niumag.com

[136] J. Mitchell and E. J. Fordham, “Contributed Review: Nuclear magnetic resonance core analysis at $0.3 \mathrm{~T}$, , Review of Scientific Instruments, vol. 85, no. 11, p. 111502, 2014.

[137] A. Legtchenko, Magnetic resonance imaging for groundwater. John Wiley \& Sons, Inc., 2013.

[138] O. a. Shushakov, "Surface NMR measurement of proton relaxation times in medium to coarse- grained sand aquifer," Magnetic Resonance Imaging, vol. 14, no. 7-8, pp. 959-960, 1996.

[139] M. Hertrich and M. Braun, "Surface nuclear magnetic resonance tomography," IEEE transactions on geosience and remote sensing, vol. 45, no. 11, pp. 3752-3759, 2007.

[140] R. Knight, E. Grunewald, T. Irons, K. Dlubac, Y. Song, H. N. Bachman, B. Grau, D. Walsh, J. D. Abraham, and J. Cannia, "Field experiment provides ground truth for surface nuclear magnetic resonance measurement," Geophysical Research Letters, vol. 39, p. L03304, Feb. 2012.

[141] M. Müller-Petke, J. O. Walbrecker, and R. Knight, “The inversion of surface-NMR data for improved aquifer characterization," Geophysics, vol. 78, no. 6, pp. EN83-EN94, 2013.

[142] A. D. Parsekian, G. Grosse, J. O. Walbrecker, M. Müller-Petke, K. Keating, L. Liu, B. M. Jones, and R. Knight, “Detecting unfrozen sediments 
below thermokarst lakes with surface nuclear magnetic resonance," Geophysical Research Letters, vol. 40, no. 3, pp. 535-540, 2013.

[143] A. a. Behroozmand, K. Keating, and E. Auken, "A Review of the Principles and Applications of the NMR Technique for Near-Surface Characterization," Surveys in Geophysics, vol. 36, no. 1, pp. 27-85, 2014.

[144] L. Drain, "The broadening of magnetic resonance lines due to field inhomogeneities in powdered samples," Proceedings of the Physical Society, vol. 1380, pp. 3-6, 1962.

[145] M. D. Hürlimann, "Effective gradients in porous media due to susceptibility differences," Journal of Magnetic Resonance, vol. 131, pp. 232240, 1998.

[146] P. N. Sen and S. Axelrod, "Inhomogeneity in local magnetic field due to susceptibility contrast," Journal of Applied Physics, vol. 86, no. 8, p. $4548,1999$.

[147] Q. Chen, A. E. Marble, B. G. Colpitts, and B. J. Balcom, "The internal magnetic field distribution, and single exponential magnetic resonance free induction decay, in rocks.," Journal of magnetic resonance, vol. 175, pp. 300-308, Aug. 2005.

[148] T. de Swiet, M. Tomaselli, M. D. Hürlimann, and A. Pines, "In Situ NMR analysis of fluids contained in sedimentary rock," Journal of Magnetic Resonance, vol. 133, pp. 385-387, 1998.

[149] T. R. Bryar, C. J. Daughney, and R. J. Knight, “Paramagnetic effects of iron(III) species on nuclear magnetic relaxation of fluid protons in porous media.," Journal of magnetic resonance, vol. 142, no. 1, pp. 74-85, 2000. 
[150] J. Mitchell, T. C. Chandrasekera, and L. Gladden, “Obtaining true transverse relaxation time distributions in high-field NMR measurements of saturated porous media: Removing the influence of internal gradients," The Journal of chemical physics, vol. 132, p. 244705, June 2010.

[151] L. J. Zielinski, "Effect of internal gradients in the nuclear magnetic resonance measurement of the surface-to-volume ratio.," The Journal of chemical physics, vol. 121, pp. 352-61, July 2004.

[152] R. Cotts, M. Hoch, T. Sun, and J. Markert, "Pulsed field gradient stimulated echo methods for improved NMR diffusion measurements in heterogeneous systems," 1989.

[153] P. Sun, J. Seland, and D. Cory, "Background gradient suppression in pulsed gradient stimulated echo measurements," Journal of Magnetic Resonance, vol. 161, pp. 168-173, Apr. 2003.

[154] P. Galvosas, F. Stallmach, and J. Kärger, "Background gradient suppression in stimulated echo NMR diffusion studies using magic pulsed field gradient ratios," Journal of Magnetic Resonance, vol. 166, pp. 164-173, Feb. 2004.

[155] G. Leu, E. J. Fordham, M. D. Hürlimann, and P. Frulla, “Fixed and pulsed gradient diffusion methods in low-field core analysis," Magnetic resonance imaging, vol. 23, no. 2, pp. 305-309, 2005.

[156] P. Sun, "Improved diffusion measurement in heterogeneous systems using the magic asymmetric gradient stimulated echo (MAGSTE) technique," Journal of Magnetic Resonance, vol. 187, no. 2, pp. 177-183, 2007.

[157] Y.-Q. Song, S. Ryu, and P. Sen, “Determining multiple length scales in rocks," Nature, vol. 407, pp. 178-181, 2000. 
[158] Y.-Q. Song, "Using internal magnetic fields to obtain pore size distributions of porous media," Concepts in Magnetic Resonance, vol. 18A, pp. 97-110, July 2003.

[159] H. Liu, L. Xiao, H. Yu, X. Li, B. Guo, Z. Zhang, F. Zong, V. Anferov, and S. Anferova, "Probing Structural Compositions of Porous Media with Two-dimensional Nuclear Magnetic Resonance," Applied Magnetic Resonance, vol. 44, pp. 543-552, Nov. 2012.

[160] J. Mitchell, "Rapid measurements of heterogeneity in sandstones using low-field nuclear magnetic resonance.," Journal of magnetic resonance, vol. 240, pp. 52-60, Mar. 2014.

[161] J. Bear, Dynamics of fluids in porous media. American Elsevier Publishing, 1972.

[162] H. Torrey, "Bloch equations with diffusion terms," Physical Review, vol. 104, no. 3, pp. 563-565, 1956.

[163] K. Brownstein and C. Tarr, "Importance of classical diffusion in NMR studies of water in biological cells," Physical Review A, vol. 19, pp. 2446-2453, 1979.

[164] D. Grebenkov, "NMR survey of reflected Brownian motion," Reviews of Modern Physics, vol. 79, no. 3, pp. 1077-1137, 2007.

[165] R. Kleinberg, W. Kenyon, and P. Mitra, "Mechanism of NMR Relaxation of Fluids in Rock," 1994.

[166] M. Hürlimann, L. Latour, and C. Sotak, "Diffusion measurement in sandstone core: NMR determination of surface-to-volume ratio and surface relaxivity," Magnetic resonance imaging, vol. 12, no. 2, pp. 325-327, 1994. 
[167] S. Roberts, P. McDonald, and T. Pritchard, "A bulk and spatially resolved NMR relaxation study of sandstone rock plugs," Journal of Magnetic Resonance, Series A, vol. 195, pp. 189-195, 1995.

[168] W. Slijkerman and J. Hofman, "Determination of surface relaxivity from NMR diffusion measurements," Magnetic resonance imaging, vol. 16, no. 98, pp. 541-544, 1998.

[169] D. J. Holland, J. Mitchell, A. Blake, and L. Gladden, "Grain Sizing in Porous Media using Bayesian Magnetic Resonance," Physical Review Letters, vol. 110, p. 018001, Jan. 2013.

[170] M. Duschl, P. Galvosas, T. I. Brox, A. Pohlmeier, and H. Vereecken, "In situ determination of surface relaxivities for unconsolidated sediments," Water Resources Research, 2015.

[171] P. P. Mitra, P. N. Sen, and L. M. Schwartz, "Short-time behavior of the diffusion coefficient as a geometrical probe of porous media," Physical Review B, vol. 47, no. 14, pp. 8565-8574, 1993.

[172] J. Mitchell, J. B. W. Webber, and J. H. Strange, "Nuclear magnetic resonance cryoporometry," Physics Reports-Review Section of Physics Letters, vol. 461, pp. 1-36, May 2008.

[173] J. Mitchell, S. C. Stark, and J. H. Strange, "Probing surface interactions by combining NMR cryoporometry and NMR relaxometry," Journal of Physics D: Applied Physics, vol. 38, pp. 1950-1958, June 2005.

[174] J. Strange, J. Mitchell, and J. Webber, "Pore surface exploration by NMR," Magnetic Resonance Imaging, vol. 21, pp. 221-226, Apr. 2003.

[175] D. Guilfoyle and P. Mansfield, "Rapid Volumetric NMR Imaging of Fluids in Porous Solids Using a 3D $\pi$ - EPI ( PEPI ) Hybrid," Journal of Magnetic Resonance, vol. 119, pp. 151-156, 1996. 
[176] M. B. Clennell, “Tortuosity: a guide through the maze," Geological Society, London, Special Publications, vol. 122, no. 1, pp. 299-344, 1997.

[177] M. Balzarini, a. Brancolini, and P. Gossenberg, "Permeability estimation from NMR diffusion measurements in reservoir rocks," Magnetic Resonance Imaging, vol. 16, no. 5-6, pp. 601-603, 1998.

[178] R. Wang, T. Pavlin, M. S. Rosen, R. W. Mair, D. G. Cory, and R. L. Walsworth, "Xenon NMR measurements of permeability and tortuosity in reservoir rocks," Magnetic Resonance Imaging, vol. 23, pp. 329-331, Feb. 2005.

[179] K. Seto, J. T. Hollenshead, A. T. Watson, C. T. Philip, C. Slattery, and J. C. Slattery, "Determination of permeability distributions using NMR velocity imaging," Transport in Porous Media, vol. 42, no. 3, pp. 351-388, 2001.

[180] J. Mitchell, M. Hürlimann, and E. Fordham, "A rapid measurement of $T_{1} / T_{2}$ : The DECPMG sequence," Journal of Magnetic Resonance, vol. 200, pp. 198-206, Oct. 2009.

[181] W. Abdallah, J. S. Buckley, A. Carnegie, J. Edwards, B. Herold, E. Fordham, et al., "Fundamentals of Wettability," Oilfield Review, pp. 44-61, 2007.

[182] J. Chen, G. Hirasaki, and M. Flaum, "NMR wettability indices: Effect of OBM on wettability and NMR responses," Journal of Petroleum Science and Engineering, vol. 52, pp. 161-171, June 2006.

[183] R. Brown and I. Fatt, "Measurements of fractional wettability of oil fields' rocks by the nuclear magnetic relaxation method," Fall Meeting of the Petroleum Branch of AIME, pp. 743-G, 1956.

[184] I. Bonalde, M. Martin-Landrove, A. Benavides, R. Martin, and J. Espidel, "Nuclear magnetic resonance relaxation study of wettability of 
porous rocks at different magnetic fields," Journal of applied physics, vol. 78 , no. 10, 1995.

[185] D. Cory, A. Guzman-Garcia, G. Leu, and P. Sen, “NMR identification of fluids and wettability in situ in preserved cores," Petrophysics, vol. 43, pp. 13-19, 2002.

[186] M. Fleury and F. Deflandre, "Quantitative evaluation of porous media wettability using NMR relaxometry," Magnetic resonance imaging, vol. 21, no. 3, pp. 385-387, 2003.

[187] J. Howard, "Quantitative estimates of porous media wettability from proton NMR measurements," Magnetic resonance imaging, vol. 16, no. 5, pp. 529-533, 1998.

[188] W. Hsu, X. Li, and R. Flumerfelt, "Wettability of porous media by NMR relaxation methods," SPE Annual Technical Conference, 1992.

[189] E. Johannesen and M. Steinsbø, "Wettability characterization by NMR $T_{2}$ measurements in chalk," SCA conference, no. 1995, pp. 1-8, 2006.

[190] G. Q. Zhang, C.-c. Huang, and G. J. Hirasaki, “Interpretation of Wettability in Sandstones With NMR Analysis '," Petrophysics, vol. 41, no. 3, pp. 223-233, 2000.

[191] J. Seland, K. Washburn, H. Anthonsen, and J. Krane, "Correlations between diffusion, internal magnetic field gradients, and transverse relaxation in porous systems containing oil and water," Physical Review E, vol. 70, p. 051305, Nov. 2004.

[192] Z. Jiang, T. Zhu, and W. Wang, "Rock core holder compatible with nuclear magnetic resonance," June 2012. CN Patent App. CN $201,110,304,182$.

[193] http://www.daedalusinnovations.com 
[194] H. Han, R. P. MacGregor, and B. J. Balcom, "Pure phase encode magnetic field gradient monitor," Journal of Magnetic Resonance, vol. 201, no. 2, pp. 212-217, 2009.

[195] H. Han and B. J. Balcom, "Magnetic resonance imaging inside metallic vessels," Measurement Science and Technology, vol. 21, no. 10, p. 103001, 2010.

[196] H. Han, D. Green, M. Ouellette, R. MacGregor, and B. J. Balcom, "Non-Cartesian sampled centric scan SPRITE imaging with magnetic field gradient and $\mathrm{B} 0(\mathrm{t})$ field measurements for MRI in the vicinity of metal structures," Journal of Magnetic Resonance, vol. 206, no. 1, pp. 97-104, 2010.

[197] H. Han, A. V. Ouriadov, E. Fordham, and B. J. Balcom, "Direct Measurement of Magnetic Field Gradient Waveforms," Concepts In Magnetic Resonance, vol. 36A, no. 6, pp. 349 - 360, 2010.

[198] H. Han, M. Ouellette, B. MacMillan, F. Goora, R. MacGregor, D. Green, and B. J. Balcom, "High pressure magnetic resonance imaging with metallic vessels," Journal of Magnetic Resonance, vol. 213, no. 1, pp. 90-97, 2011.

[199] H. Han and B. J. Balcom, "Magnetic resonance imaging inside cylindrical metal containers with an eddy current self-compensated method," Measurement Science and Technology, vol. 22, no. 11, p. 115501, 2011.

[200] P. Mansfield and B. Issa, "A microscopic model of fluid transport in porous rocks," Magnetic Resonance Imaging, vol. 14, no. 7-8, pp. 711714, 1996.

[201] M. L. Johns, a. J. Sederman, L. Gladden, a. Wilson, and S. Davies, "Using MR techniques to probe permeability reduction in rock cores," AIChE Journal, vol. 49, pp. 1076-1084, May 2003. 
[202] J. Mitchell, A. J. Sederman, E. Fordham, M. L. Johns, and L. Gladden, "A rapid measurement of flow propagators in porous rocks.," Journal of magnetic resonance (San Diego, Calif. : 1997), vol. 191, pp. 267-72, Apr. 2008.

[203] P. Singer, G. Leu, E. Fordham, and P. Sen, “Low magnetic fields for flow propagators in permeable rocks," Journal of Magnetic Resonance, vol. 183, pp. 167-77, Dec. 2006.

[204] D. Verganelakis, J. Crawshaw, M. Johns, M. Mantle, U. Scheven, A. Sederman, and L. Gladden, "Displacement propagators of brine flowing within different types of sedimentary rock," Magnetic resonance imaging, vol. 23, pp. 349-351, Feb. 2005.

[205] W. Zhao, G. Picard, G. Leu, and P. M. Singer, "Characterization of Single-Phase Flow Through Carbonate Rocks: Quantitative Comparison of NMR Flow Propagator Measurements with a Realistic Pore Network Model," Transport in Porous Media, vol. 81, pp. 305-315, May 2009 .

[206] R. Kulkarni and A. Watson, "Estimation of multiphase flow functions from dynamic displacement data: applications of NMR imaging," Society of Petroleum Enginnering, pp. 357-362, 1996.

[207] R. Kulkarni, A. Watson, and J. Nordtvedt, "Estimation of porous media flow functions using NMR imaging data," Magnetic resonance imaging, vol. 16, no. 98, pp. 707-709, 1998.

[208] J. Mitchell, a.M. Howe, and a. Clarke, "Real-time oil-saturation monitoring in rock cores with low-field NMR," Journal of Magnetic Resonance, vol. 256, no. April, pp. 34-42, 2015.

[209] M. Enwere and J. Archer, "NMR Imaging for Core Flood Testing," International Symposium of the Society of Core Analysis, no. 92, p. 1992, 1992. 
[210] C. Songhua, F. Qin, K.-H. Kim, and A. Waton, "NMR imaging of multiphase flow in porous media," Society of petroleum engineering, 1993.

[211] Q. Chen, W. Kinzelbach, and O. Sascha, "Nuclear magnetic resonance imaging for studies of flow and transport in porous media," Journal of environmental quality, pp. 477-486, 2002.

[212] L. F. Gladden, "Nuclear magnetic resonance in chemical engineering: principles and applications," Chemical engineering science, vol. 49, no. 20, pp. 3339-3408, 1994.

[213] E. Fordham, M. Horsfield, C. Hall, and L. Hall, "Low-contrast secondary imbibition in long rock cores," Magnetic resonance imaging, 1991.

[214] L. Li, Q. Chen, A. E. Marble, L. Romero-Zerón, B. Newling, and B. J. Balcom, "Flow imaging of fluids in porous media by magnetization prepared centric-scan SPRITE.," Journal of Magnetic Resonance, vol. 197, pp. 1-8, Mar. 2009.

[215] L. F. Gladden and A. J. Sederman, "Recent advances in Flow MRI," Journal of Magnetic Resonance, vol. 229, pp. 2-11, 2013.

[216] J. C. Stevens, J. J. Howard, and B. a. Baldwin, "Experimental hydrate formation and gas production scenarios based on $\mathrm{CO}_{2}$ sequestration," Proceedings of the 6th International Conference on Gas Hydrates, 2008.

[217] A. Graue, "Mechanistic Fluid Flow Dynamics in Heterogeneous Porous Rocks Determined by Complementary In-Situ Imaging Techniques," 5th WSEAS International Conference on Fluid Mechanics, 2008.

[218] G. Ersland, J. Husebø, a. Graue, B. a. Baldwin, J. Howard, and J. Stevens, "Measuring gas hydrate formation and exchange with 
$\mathrm{CO}_{2}$ in Bentheim sandstone using MRI tomography," Chemical Engineering Journal, vol. 158, no. 1, pp. 25-31, 2010.

[219] Y. Zhao, Y. Song, T. Wang, Y. Liu, L. Jiang, N. Zhu, and W. Yang, "Visualisation of Water Flooding and Subsequent Supercritical $\mathrm{CO}_{2}$ Flooding in Fractured Porous Media with Permeability Heterogeneity Using MRI," Energy Procedia, vol. 37, pp. 6942-6949, 2013.

[220] H. Riskedal, L. Tipura, J. Howard, and A. Graue, "NMR monitoring of spontaneous brine imbibition in carbonates," International Symposium of the Society of Core Analysis, pp. 1-12, 2008.

[221] E. Toumelin, C. Torres-Verdín, B. Sun, and K. J. Dunn, "Random-walk technique for simulating NMR measurements and 2D NMR maps of porous media with relaxing and permeable boundaries," Journal of Magnetic Resonance, vol. 188, no. 1, pp. 83-96, 2007.

[222] C. Arns, T. AlGhamdi, and J. Arns, "Numerical analysis of nuclear magnetic resonance relaxation-diffusion responses of sedimentary rock," New Journal of Physics, vol. 13, no. 1, p. 015004, 2011.

[223] D. Grebenkov, "Laplacian eigenfunctions in NMR. I. A numerical tool," Concepts in Magnetic Resonance Part A, vol. 32, no. 4, pp. 277-301, 2008.

[224] Y.-Q. Song, "Detection of the high eigenmodes of spin diffusion in porous media," Physical Review Letters, vol. 85, pp. 3878-3881, Oct. 2000.

[225] N. V. Lisitza and Y.-Q. Song, "The behavior of diffusion eigenmodes in the presence of internal magnetic field in porous media," The Journal of Chemical Physics, vol. 114, no. 20, p. 9120, 2001.

[226] Y.-Q. Song, H. Cho, T. Hopper, A. E. Pomerantz, and P. Z. Sun, “Magnetic resonance in porous media: recent progress," The Journal of Chemical Physics, vol. 128, p. 052212, Feb. 2008. 
[227] Y.-Q. Song, L. Zielinski, and S. Ryu, "Two-Dimensional NMR of Diffusion Systems," Physical Review Letters, vol. 100, p. 248002, June 2008.

[228] L. Zielinski, R. Ramamoorthy, C. Minh, K. Daghar, and R. Sayed, "Restricted Diffusion Effects in Saturation Estimates from 2D DiffusionRelaxation NMR Maps," SPE Annual Technical Conference and Exhibition, pp. 1-8, 2010.

[229] Z.-X. Luo, J. Paulsen, and Y.-Q. Song, "Robust determination of surface relaxivity from nuclear magnetic resonance $D-T_{2}$ measurements," Journal of Magnetic Resonance, vol. 259, pp. 146-152, 2015.

[230] J. Kärger, H. Pfeifer, and S. Rudtsch, "The influence of internal magnetic field gradients on NMR self-diffusion measurements of molecules adsorbed on microporous crystallites," Journal of Magnetic Resonance, vol. 387, pp. 381-387, 1989.

[231] D. S. Grebenkov, "From the microstructure to diffusion MRI, and back," pmc.polytechnique.fr, pp. 1-65, 2015.

[232] D. Canet, J. Brondeau, and K. Elbayed, "Superfast $T_{1}$ determination by inversion-recovery," Journal of Magnetic Resonance, vol. 490, pp. 483-490, 1988.

[233] J.-J. Hsu and I. J. Lowe, "Spin-lattice relaxation and a fast $T_{1}$-map acquisition method in MRI with transient-state magnetization," Journal of Magnetic Resonance, vol. 169, no. 2, pp. 270-278, 2004.

[234] S. Ahola and V.-V. Telkki, “Ultrafast two-dimensional NMR relaxometry for investigating molecular processes in real time.," Chemphyschem : a European journal of chemical physics and physical chemistry, vol. 15, pp. 1687-92, June 2014. 
[235] W. Kenyon, H. Takezaki, C. Straley, P. Sen, M. Herron, A. Matteson, M. Petricola, et al., "A laboratory study of nuclear magnetic resonance relaxation and its relation to depositional texture and petrophysical properties-carbonate thamama group, mubarraz field, abu dhabi," SPE-29886-MS, 1995.

[236] I. Shikhov and C. Arns, "Spatially resolved relative permeability with simulated and experimental 1D NMR imaging," ICMRM 12th, London, 2012.

[237] P. R. King, "The use of renormalization for calculating effective permeability," Transport in Porous Media, vol. 4, no. 1, pp. 37-58, 1989.

[238] C. Arns, "An analysis of NMR-permeability scaling rules by numerical MRI," SPWLA Annual Logging Symposium, vol. 48, pp. 1-14, 2007.

[239] C. Green and L. Paterson, "Analytical three-dimensional renormalization for calculating effective permeabilities," Transport in Porous Media, vol. 68, no. 2, pp. 237-248, 2007.

[240] H. Liu, M. Hunter, S. Obruchkov, E. McCarney, M. Bobinson, R. Dykstra, and P. Galvosas, "Characterizing fluid presence and transport in rock cores ar reservoir-like conditions via spatially resolved NMR relaxation/diffusion maps," SCA Conference Paper, pp. 1-12, 2015.

[241] A. S. Cherkaoui and W. S. Wilcock, "Laboratory studies of high rayleigh number circulation in an open-top hele-shaw cell: An analog to mid-ocean ridge hydrothermal systems," Journal of Geophysical Research: Solid Earth (1978-2012), vol. 106, no. B6, pp. 10983-11000, 2001.

[242] J. Perlo, F. Casanova, and B. Blümich, "3D imaging with a singlesided sensor: an open tomograph," Journal of Magnetic Resonance, vol. 166, no. 2, pp. 228-235, 2004. 
[243] O. V. Petrov, G. Ersland, and B. J. Balcom, " $T_{2}$ distribution mapping profiles with phase-encode MRI," Journal of Magnetic Resonance, vol. 209, pp. 39-46, Mar. 2011.

[244] D. Green, D. Veselinovic, B. Balcom, and F. Marica, "Applications of a new technique to acquire spatially resolved NMR petrophysical data," SCA Conference Paper, pp. 1-12, 2012.

[245] D. E. Freed, "Temperature and pressure dependence of the diffusion coefficients and NMR relaxation times of mixtures of alkanes," The Journal of Physical Chemistry B, vol. 113, no. 13, pp. 4293-4302, 2009.

[246] D. E. Freed and M. D. Hürlimann, "One-and two-dimensional spin correlation of complex fluids and the relation to fluid composition," Comptes Rendus Physique, vol. 11, no. 2, pp. 181-191, 2010.

[247] M. Holz, S. R. Heil, and A. Sacco, "Temperature-dependent selfdiffusion coefficients of water and six selected molecular liquids for calibration in accurate ${ }^{1} \mathrm{H}$ NMR PFG measurements," Physical Chemistry Chemical Physics, vol. 2, no. 20, pp. 4740-4742, 2000.

[248] R. T. Lewis, K. Djurhuus, and J. G. Seland, “Characterising oil and water in porous media using decay due to diffusion in the internal field," Journal of Magnetic Resonance, vol. 259, pp. 1-9, 2015.

[249] D. Xiao and B. J. Balcom, "k-t Acceleration in pure phase encode MRI to monitor dynamic flooding processes in rock core plugs," Journal of Magnetic Resonance, vol. 243, pp. 114-121, 2014.

[250] D. Xiao and B. J. Balcom, " $\pi$ Echo-Planar Imaging with concomitant field compensation for porous media MRI," Journal of Magnetic Resonance, vol. 260, pp. 38-45, 2015. 